, 
Printed at the Mathematical Centre, 49, 2e Boerhaavestraat, Amsterdam.

The Mathematical Centre, founded the 11-th of February 1946, is a nonprofit institution aiming at the promotion of pure mathematics and its applications. It is sponsored by the Netherlands Government through the Netherlands Organization for the Advancement of Pure Research $(2 . W .0)$. 
MATHEMATICAL CENTRE TRACTS 98

\section{SINGULAR PERTURBATIONS OF HYPERBOLIC TYPE}

R. GEEL

MATHEMATISCH CENTRUM AMSTERDAM 1978 
AMS (MOS) Classification scheme (1970): 34E05, 34E15, 35B25, 35B45, $35 \mathrm{C} 10,35 \mathrm{~L} 15,35 \mathrm{~L} 20,35 \mathrm{~L} 60$.

ISBN 9061961661 
CONTENTS

CHAPTER I INITIAL VALUE PROBLEMS FOR LINEAR ORDINARY DIFFERENTIAL EQUATIONS

1. A priori estimates

2. Asymptotic expansions

3. The case $a(t ; \varepsilon) \leq-a_{0}<0$

4. Discussion

CHAPTER II INITIAL VALUE PROBLEMS FOR NONLINEAR ORDINARY DIFFERENTIAL EQUATIONS

1. Existence and a priori estimates

2. Asymptotic expansions

CHAPTER III INITIAL VALUE PROBLEMS FOR LINEAR HYPERBOLIC DIFFERENTIAL EQUATIONS

1. Preliminaries

2. A priori estimates

3. Asymptotic expansions

4. Discussion

CHAPTER IV INITIAL VALUE PROBLEMS FOR NONLINEAR HYPERBOLIC DIFFERENTIAL EQUATIONS

1. Existence and a priori estimates

2. Asymptotic expansions

3. Generalized solutions of the reduced problem

4. Problems with $a$ and $b$ independent of $u$

4.1. The case $d(x, t, u)=d(x, t) \sin u$

4.2. The case $d(x, t, u)=d_{1}(x, t) u+d_{2}(x, t) u|u|^{\nu}$ 
CHAPTER V INITIAL - BOUNDARY VALUE PROBLEMS FOR LINEAR

HYPERBOLIC DIFFERENTIAL EQUATIONS 121

1. A priori estimates 123

2. Approximations 134

2.1. A lemma 136

2.2. Construction of an approximation 143

CHAPTER VI CHARACTERISTIC BOUNDARY VALUE PROBLEMS FOR LINEAR HYPERBOLIC DIFFERENTIAL EQUATIONS 155

1. A priori estimates 156

2. Approximations for the case without boundary layer along $\mathrm{x}=\phi(\mathrm{y}), \mathrm{y} \geq 0$

3. Approximations for the case with boundary

layer along $\mathrm{x}=\phi(\mathrm{y}), \mathrm{y} \geq 0$ 


\section{ACKNOWLEDGEMENTS}

It is with great pleasure that I thank Prof. E.M. de Jager for introducing me to the field of singular perturbations and for guiding me. His continuous interest in my work, his critical and stimulating remarks and above all his enthusiasm were very inspiring.

I owe much to Prof. W. Eckhaus for his valuable advice and criticism.

I thank the Mathematical Centre for the opportunity to publish this monograph in their series Mathematical Centre Tracts and all those at the Mathematical Centre who have contributed to its technical realization. 

INTRODUCTION

This work deals with initial value problems and initial-boundary value problems in $\mathbb{I R}^{2}$ which are governed by a differential equation of the type

$$
L^{(\varepsilon)}[u] \equiv \varepsilon L_{2}[u]+L_{1}[u]=f(x, t)
$$

where $\varepsilon$ is a small positive parameter (i.e. $0<\varepsilon<1$ ), the operator $\mathrm{I}_{2}$ is a linear hyperbolic differential operator of the second order given by

$$
L_{2}[u]=\frac{\partial^{2} u}{\partial t^{2}}+\left\{c_{1}(x, t)+c_{2}(x, t)\right\} \frac{\partial^{2} u}{\partial x \partial t}+c_{1}(x, t) c_{2}(x, t) \frac{\partial^{2} u}{\partial x^{2}}
$$

(with $c_{1}<c_{2}$ ) and the operator $I_{1}$ is a nonvanishing first order differential operator which is either linear

$$
L_{1}[u]=a(x, t) \frac{\partial u}{\partial t}+b(x, t) \frac{\partial u}{\partial x}+d(x, t) u
$$

or quasilinear

$$
L_{1}[u]=a(x, t, u) \frac{\partial u}{\partial t}+b(x, t, u) \frac{\partial u}{\partial x}+d(x, t, u) .
$$

The equation (1) describes the propagation of waves and occurs in many problems in mathematical physics. One class of physical problems which lead to the equation (1) concerns certain phenomena which are governed in first approximation by a quasilinear conservation law of the type

$$
\frac{\partial u}{\partial t}+\phi(u) \frac{\partial u}{\partial x}=0
$$

If higher order effects are taken into account there results a nonlinear mathematical model which yields after linearization an equation of the type (1). Phenomena which can thus be described are for instance traffic flow, the flow of water in long rivers and glacier flow. Also certain chemical exchange processes and the process of sedimentation in rivers are governed by a conservation law of the above mentioned type. For details we refer to WHITHAM [56, chapter 3] where also an extensive bibliography can be found. Other physical problems which lead to the equation (1) are so-called 
$\mathrm{vi}$

overdamped vibration problems. Problems of this kind are governed by an equation of the type

$$
L_{2}[u]=\mu\left\{f(x, t)-L_{1}[u]\right\}
$$

where $\mu$ is some large positive constant (i.e. $\mu>>1$ ) and $L_{2}$ and $L_{1}$ are given by (2) and (3). Putting $\mu=\varepsilon^{-1}$ in (5) we obtain the equation (1). Physically the large constant $\mu$ corresponds to a strong suppression of the vibration which may be caused for instance by a heavy damping (mechanical problems) or by a highly absorbing surrounding medium (radiation problems). An example of an overdamped vibration is given by the propagation of electrical signals along a conducting wire of large resistance. In this case the mathematical description leads to the well-known telegraph equation with a small factor $\varepsilon=\mu^{-1}$ in front of the wave operator (see SMITH \& PALMER [46]). Other overdamped vibration problems are for instance the motion of a vibrating string imbedded in a highly viscous medium and the propagation of radiation through a highly absorbing medium.

Since problems which are governed by an equation of the type (1) belong to the class of so-called singular perturbation problems we shall give a brief description of the concept of singular perturbation problem. This description will be followed by a discussion of some techniques which are characteristic of singular perturbation theory.

Let $\Omega$ be some domain in $\mathbb{I R}^{\mathrm{n}}$ with boundary $\partial \mathrm{D}$ and let $\vec{x}=\left(\mathrm{x}_{1}, \mathrm{x}_{2}, \ldots, \mathrm{x}_{\mathrm{n}}\right)$ denote a point in $\mathbb{I R}^{\mathrm{n}}$. We consider the boundary value problem

$$
\left\{\begin{array}{l}
L^{(\varepsilon)}[u] \equiv \varepsilon L_{2}[u]+L_{1}[u]=f(\vec{x}), \quad \vec{x} \in \Omega \\
B_{i}[u]=g_{i}(\vec{x}) \quad \text { along } \partial D(i=1,2, \ldots, k)
\end{array}\right.
$$

where $f$ and $g_{i}(i=1,2, \ldots, k)$ are given functions and the $B_{i}[u]$ $(i=1,2, \ldots, \mathrm{k})$ are expressions involving $u$ and its derivatives; the constant $\mathrm{k}$ is a positive integer depending on the type of boundary value problem. $\mathrm{L}_{2}$ and $\mathrm{L}_{1}$ are differential operators of arbitrary type (either ordinary differential operators or partial differential operators of elliptic, parabolic or hyperbolic type) and of arbitrary order; these operators 
may be linear or nonlinear. The constant $\varepsilon$ is a small positive parameter which appears only as a factor in front of the operator $\mathrm{L}_{2}$.

One can think of the left-hand side of the differential equation (6) as consisting of a term $L_{1}[u]$ together with a small perturbation $\varepsilon L_{2}[u]$. For this reason problems of the type (6) are called perturbation problems; the term $L_{1}[\mathrm{u}]$ is referred to as the unperturbed $\varepsilon$-independent part of the operator $I_{J}(\varepsilon)$ and the term $\varepsilon L_{2}[u]$ is called the perturbation.

One distinguishes between regular perturbation problems and singular perturbation problems. A class of singular perturbation problems is characterized by the fact that the order of $L_{2}$ is larger than the order of $L_{1}$; in case the order of $\mathrm{L}_{2}$ is lower than or equal to that of $\mathrm{L}_{1}$ we speak of regular perturbation problems (for an analysis of the concepts regular perturbation versus singular perturbation we refer to ECKHAUS [10]). From now on we shall assume that (6) is a singular perturbation problem in which the order of $\mathrm{L}_{2}$ is larger than that of $\mathrm{L}_{1}$.

A solution $u$ of problem (6) is in general difficult to obtain explicitly. In practice, however, it often occurs that one is content with an approximation $\tilde{u}$ of $u$ which is valid for small values of $\varepsilon$. In that case the problem reduces to the determination of a function $\tilde{u}$ which satisfies

$$
\lim _{\varepsilon \downarrow 0}\|u-\tilde{u}\|_{\bar{\Omega}}=0
$$

Here $\|\cdot\|_{\bar{\Omega}}$ denotes some norm which has been given in advance. We may take for instance the sup-norm

$$
\|u-\tilde{u}\|_{\bar{\Omega}}=\sup _{\bar{\Omega}}|u-\tilde{u}|
$$

or the $\mathrm{L}_{2}$-norm

$$
\|u-\tilde{u}\|_{\bar{\Omega}}=\left[\int_{\bar{\Omega}}|u-\tilde{u}|^{2} d \vec{x}\right]^{\frac{1}{2}}
$$

where $\bar{\Omega}$ denotes the closure of $\Omega$; also norms involving derivatives are possible. The construction of the approximation $\tilde{u}$ is usually carried out in two steps. First one constructs a so-called formal approximation $\tilde{u}$ which satisfies the differential equation and the boundary conditions (6) up to 
VIII

some order of $\varepsilon$. In a second step one proves the correctness of the formal approximation by showing that (7) holds.

We shall now discuss both steps in some more detail, where it will be assumed for simplicity that the operators $L_{1}$ and $L_{2}$, as well as the expressions $B_{i}(i=1,2, \ldots, k)$, are linear. However, much of what will be said applies also to nonlinear problems.

One always starts the construction of the formal approximation by solving the reduced equation

$$
L_{1}\left[w_{0}\right]=f(\vec{x}), \quad \vec{x} \in \Omega
$$

which follows from the equation (6) by putting $\varepsilon=0$. The solution $\mathrm{w}_{0}$ is chosen such that it satisfies as many of the boundary conditions (6) as possible (the reduced equation together with the selected boundary conditions is also referred to as the reduced problem). If $w_{0}$ is sufficiently smooth it satisfies the differential equation (6) up to order $\varepsilon$, namely

$$
L^{(\varepsilon)}\left[\mathrm{w}_{0}\right]=\mathrm{f}(\overrightarrow{\mathrm{x}})+\varepsilon \mathrm{L}_{2}\left[\mathrm{w}_{0}\right]=\mathrm{f}(\overrightarrow{\mathrm{x}})+0(\varepsilon), \quad \varepsilon \downarrow 0
$$

uniformly valid in subsets of $\Omega$. However, since the order of $\mathrm{L}_{2}$ is larger than that of $L_{1}$ the function $w_{0}$ generally does not satisfy all boundary conditions (6), so $\mathrm{w}_{0}$ is not a formal approximation of $u$.

In order to cope with the remaining boundary conditions (6) a so-called boundary layer function $\mathrm{v}_{0}$ is added to $\mathrm{w}_{0}$ which, for ' $\varepsilon$ approaching zero, tends asymptotically to zero everywhere in $\Omega$ with the exception of a small neighborhood of the boundary $\partial \Omega$ where it contributes to $\mathrm{w}_{0}$ in such a way that the remaining boundary conditions are fulfilled; the appearance of boundary layers is characteristic of singular perturbation problems. Usually a boundary layer function can be constructed by stretching the coordinate, say $x_{n}$ ' which measures the distance of a point $\vec{x}$ to the boundary (if necessary, one introduces local coordinates in a neighborhood of the boundary). The following type of boundary layer function occurs frequently in singular perturbation problems:

$$
v_{0}=v_{0}\left(x_{1}, x_{2}, \ldots, x_{n-1}, \frac{x_{n}}{\varepsilon^{p}}\right)=\phi\left(x_{1}, \ldots, x_{n-1}\right) \exp \left(-\frac{x_{n}}{\varepsilon^{p}}\right), \quad x_{n} \geq 0
$$


where $\mathrm{p}$ is some positive constant.

Once a boundary layer function $\mathrm{v}_{0}$ has been determined a formal approximation $\tilde{u}$ of $u$ (up to order $\varepsilon$ ) can be written down, namely

$$
\tilde{u}=w_{0}+v_{0}
$$

Formal approximations of higher order can be obtained by taking more terms into account. For instance, the function

$$
\tilde{u}=\sum_{k=0}^{n} \varepsilon^{k} w_{k}+\sum_{k=0}^{n} \varepsilon^{k} v_{k}
$$

with an appropriate choice for the functions $\mathrm{w}_{\mathrm{k}}(\mathrm{k}=0,1, \ldots, \mathrm{n})$ and the boundary layer functions $v_{k}(k=0,1, \ldots, n)$ may give a formal approximation of $u$ up to order $\varepsilon^{n+1}$.

As an illustration we shall now construct a formal approximation for the solution of a simple initial value problem in one independent variable, namely

$$
\left\{\begin{array}{l}
L^{(\varepsilon)}[u] \equiv \varepsilon \frac{d^{2} u}{d t^{2}}+\frac{d u}{d t}+u=f(t), \quad 0<t<\infty \\
u(0 ; \varepsilon)=C_{1}, \quad u_{t}(0 ; \varepsilon)=C_{2}
\end{array}\right.
$$

with $f$ some $C^{\infty}$-function. This problem can of course easily be solved explicitly, but we give this example in this introduction, merely to illustrate the construction of a formal approximation. Stretching the coordinate $t$ by introducing

$$
\tau=\frac{t}{\varepsilon}
$$

we can write the differential operator (9) as

$$
L^{(\varepsilon)}[u] \equiv \varepsilon^{-1}\left(\frac{d^{2} u}{d \tau^{2}}+\frac{d u}{d \tau}\right)+u .
$$

Let $\tilde{u}$ be defined by (8) where the functions $w_{k}$ depend on $t$ and the boundary layer functions $\mathrm{v}_{\mathrm{k}}$ depend on $\tau$. Then we obtain 
$\mathbf{x}$

(10)

$$
\begin{aligned}
L^{(\varepsilon)}[\tilde{u}]= & L^{(\varepsilon)}\left[\sum_{k=0}^{n} \varepsilon^{k} w_{k}\right]+L^{(\varepsilon)}\left[\sum_{k=0}^{n} \varepsilon^{k} v_{k}\right] \\
= & \left.\frac{d w_{0}}{d t}+w_{0}+\varepsilon^{-1} \frac{d^{2} v_{0}}{d \tau^{2}}+\frac{d v_{0}}{d \tau}\right) \\
& +\sum_{k=1}^{n} \varepsilon^{k}\left(\frac{d w_{k}}{d t}+w_{k}+\frac{d^{2} w_{k-1}}{d t^{2}}\right) \\
& \left.+\sum_{k=1}^{n} \varepsilon^{k-1} \frac{d^{2} v_{k}}{d \tau^{2}}+\frac{d v_{k}}{d \tau}+v_{k-1}\right) \\
& +\varepsilon^{n+1} \frac{d^{2} w_{n}}{d t^{2}}+\varepsilon^{n} v_{n}
\end{aligned}
$$

and also

$$
\left\{\begin{array}{l}
\tilde{u}(0 ; \varepsilon)=w_{0}(0)+\sum_{k=1}^{n} \varepsilon^{k}\left\{w_{k}(0)+\varepsilon^{-1} v_{k-1}(0)\right\}+\varepsilon^{n} v_{n}(0) \\
\tilde{u}_{t}(0 ; \varepsilon)=\sum_{k=0}^{n} \varepsilon^{k}\left\{\frac{d w_{k}}{d t}(0)+\varepsilon^{-1} \frac{d v_{k}}{d \tau}(0)\right\} .
\end{array}\right.
$$

The functions $w_{k}(k=0,1, \ldots, n)$ and $v_{k}(k=0,1, \ldots, n)$ can now be obtained by solving the following set of initial value problems

(12)

$$
\begin{aligned}
& \left\{\begin{array}{l}
\frac{d w_{0}}{d t}+w_{0}=f(t), \quad 0<t<\infty \\
w_{0}(0)=c_{1}
\end{array}\right. \\
& \left\{\begin{array}{l}
\frac{d^{2} v_{0}}{d \tau^{2}}+\frac{d v_{0}}{d \tau}=0, \quad 0<\tau<\infty \\
\frac{d v_{0}}{d \tau}(0)=\varepsilon\left\{c_{2}-\frac{d w_{0}}{d t}(0)\right\} \\
\lim _{\tau \rightarrow \infty} v_{0}(\tau)=0
\end{array}\right.
\end{aligned}
$$

and for $\mathrm{k}=1,2, \ldots, \mathrm{n}$ 
(14)

$$
\begin{aligned}
& \left\{\begin{array}{l}
\frac{d w_{k}}{d t}+w_{k}=-\frac{d^{2} w_{k-1}}{d t^{2}}, \quad 0<t<\infty \\
w_{k}(0)=-\varepsilon^{-1} v_{k-1}(0)
\end{array}\right. \\
& \left\{\begin{array}{l}
\frac{d^{2} v_{k}}{d \tau^{2}}+\frac{d v_{k}}{d \tau}=-v_{k-1} \\
\frac{d v_{k}}{d \tau}(0)=-\varepsilon \frac{d w_{k}}{d t}(0) \\
\lim _{\tau \rightarrow \infty} v_{k}(\tau)=0 .
\end{array}\right.
\end{aligned}
$$

It is easily verified that the functions $v_{k}$ are of the type

$$
v_{k}\left(\frac{t}{\varepsilon}\right)=\varepsilon P_{k}\left(\frac{t}{\varepsilon}\right) \exp \left(-\frac{t}{\varepsilon}\right)
$$

with $\mathrm{P}_{k}(t)$ some polynomial in $t_{\text {; }}$ these functions are typical boundary layer functions. Moreover it follows from (10), (11), (12), (13), (14) and (15) that

$$
\left\{\begin{array}{l}
L^{(\varepsilon)}[\tilde{u}]=f(t)+0\left(\varepsilon^{n+1}\right), \quad 0<t<\infty \\
\tilde{u}(0 ; \varepsilon)=C_{1}+0\left(\varepsilon^{n+1}\right) \\
\tilde{u}_{t}(0 ; \varepsilon)=C_{2}
\end{array}\right.
$$

so $\tilde{u}$ is a formal approximation (up to order $\varepsilon^{n+1}$ ) for the solution $u$ of (9). For an exposition of formal constructive methods we refer to COLE [6] and NAYFEH [32]. FRIEDRICHS [14] has written a survey article on the occurrence of boundary layers in various branches of mathematical physics. A generalization of the concept of formal approximation can be found in ECKHAUS [11].

After a formal approximation up to some order of $\varepsilon$, say $\varepsilon^{n+1}$, has been constructed its correctness (that is, the validity of (7)) remains to be 
shown. This can be done by introducing the so-called remainder term $\mathrm{z}$ defined by

$$
\mathrm{z}=\mathrm{u}-\tilde{\mathrm{u}}
$$

and proving that

$$
\lim _{\varepsilon \downarrow 0}\|z\|_{\bar{\Omega}}=0
$$

Since $\tilde{u}$ is a formal approximation of $u$ up to order $\varepsilon^{n+1}$ it follows that $z$ satisfies a boundary value problem of the type

$$
\left\{\begin{array}{l}
\mathrm{L}^{(\varepsilon)}[\mathrm{z}]=\mathrm{L}^{(\varepsilon)}[\mathrm{u}]-\mathrm{L}^{(\varepsilon)}[\tilde{\mathrm{u}}]=0\left(\varepsilon^{\mathrm{n}+1}\right), \quad \overrightarrow{\mathrm{x}} \in \Omega \\
\mathrm{B}_{i}[\mathrm{z}]=0\left(\varepsilon^{\mathrm{n}+1}\right) \text { along } \partial \Omega(i=1,2, \ldots, \mathrm{k})
\end{array}\right.
$$

so in order to prove (17) it suffices to derive an a priori estimate for the solution $z$ of (18) which estimates $\left\|_{z}\right\|_{\bar{\Omega}}$ in terms of the right-hand side function and the boundary functions. Once an a priori estimate has been established, say for instance

$$
\left\|_{z}\right\|_{\bar{\Omega}}=0\left(\varepsilon^{n+1}\right), \quad \varepsilon+0
$$

we obtain from (8) and (16)

$$
\left\|_{u}-\sum_{k=0}^{n} \varepsilon^{k}\left(w_{k}+v_{k}\right)\right\|_{\bar{\Omega}}=0\left(\varepsilon^{n+1}\right), \quad \varepsilon \downarrow 0
$$

from which the correctness of the formal approximation follows. The series in the left-hand side of (19) is called an asymptotic expansion of u into powers of $\varepsilon$.

REMARK. Not every formal approximation is a correct approximation. Examples of formal approximations which are not approximations can be found for instance in ECKHAUS [11] and will also be given in this thesis.

A priori estimates for the solution $\mathrm{z}$ of (18) can be obtained by various methods, depending on the nature of the operators $\mathrm{L}_{1}$ and $\mathrm{L}_{2}$. For problems with a singular perturbation of elliptic type (that is, for problems 
with an elliptic operator $\mathrm{L}_{2}$ ) a priori estimates have been established amongst others by BESJES [2], ECKHAUS \& DE JAGER [12], VAN HARTEN [23], HUET $\left[24^{*}\right]$, LIONS [28] and VISIK \& LYUSTERNIK [49], whereas a priori estimates for problems with a singular perturbation of parabolic type can be found for instance in BESJES [2] and LIONS [28]. In this thesis we shall be concerned primarily with singular perturbation problems in $\mathbb{R}^{2}$ which are governed by an equation of the type (1) in which the operator $\mathrm{L}_{2}$ is a linear hyperbolic differential operator of the second order. A priori estimates for solutions of these kind of problems can be obtained by applying the so-called energy integral method. This method can briefly be described as follows:

consider the initial-boundary value problem

$$
\left\{\begin{array}{l}
L_{2}[u]+L_{1}[u]=f(x, t) \quad \text { in } \Omega \subset \mathbb{R}^{2} \\
u \text { or } u \text { and its first normal derivative prescribed along } \partial \Omega
\end{array}\right.
$$

where the operators $L_{2}$ and $L_{1}$ are given by (2) and (3) (in order to fix the idea one may think of $\mathrm{L}_{2}$ as the wave operator and take for $\Omega$ the strip $0<\mathrm{x}<1,0<\mathrm{t}<\infty$; in that case $\mathrm{u}$ should be prescribed along $\mathrm{x}=0$, $t \geq 0$ and $x=1, t \geq 0$, whereas $u$ and $u_{t}$ should be prescribed along $t=0$, $0 \leq \mathrm{x} \leq 1)$. Note that $(20)$ is not a singular perturbation problem. We multiply the differential equation by a linear combination of $u(x, t)$ and its first derivatives and integrate the resulting formula over a suitably chosen region $S \subset \bar{\Omega}$, which usually contains a part of the boundary $\partial \Omega$. This yields

$$
\iint_{S}\left(\alpha u_{t}+\beta u_{x}+\gamma u\right)\left(L_{2}[u]+L_{1}[u]\right) d x d t=\iint_{S}\left(\alpha u_{t}+\beta u_{x}+\gamma u\right) f d x d t .
$$

Selecting the coefficients $\alpha=\alpha(x, t), \beta=\beta(x, t)$ and $\gamma=\gamma(x, t)$ in an appropriate way, applying GREEN's theorem in the plane to the left-hand side integral and taking into account the prescribed initial and boundary conditions we obtain certain integrals of positive definite quadratic forms in $u, u_{x}$ and $u_{t}$, usually referred to as energy integrals. A careful manipulation of these energy integrals leads then to an a priori estimate for the solution $u$ of $(20)$.

The energy integral method has been known for a long time as a powerful tool 
xุı

for obtaining a priori estimates and uniqueness proofs for solutions of initial value problems, not only in $\mathbb{R}^{2}$ but also in $\mathbb{R}^{\mathrm{n}}$ (see for instance HADAMARD [22], RUBINOWICZ [42], WEBER [53], ZAREMBA [57] and the review in the article by FRIEDRICHS [13]). In its simplest form, with the choice

$$
\alpha \equiv 1, \beta \equiv \gamma \equiv 0
$$

the method has been applied to CAUCHY's problem for linear hyperbolic equations of the second order (see FRIEDRICHS \& LEWY [15]); a less trivial choice of $\alpha, \beta$ and $\gamma$ is required for a proof of the uniqueness of the solution of TRICOMI's problem (cf. PROTTER [40]) or FRANKL's problem (cf. MORAWETZ [31]). Although the energy integral method is usually applied to hyperbolic equations it has also been used for elliptic equations (see GARABEDIAN $[16, \mathrm{p} .426])$.

If the energy integral method descrived above is applied to singularly perturbed initial value problems which are governed by an equation of the type (1), (2), (3) a slight complication is formed by the presence of the small factor $\varepsilon$ in front of the hyperbolic operator $L_{2}$ since it turns out that due to this factor the choice (21) for $\alpha, \beta$ and $\gamma$ does no longer lead to useful estimates (see DE JAGER [26]). In case the coefficient $d(x, t)$ in (3) is identically equal to zero this difficulty can be overcome by choosing $\alpha$ and $\beta$ such that

$$
\alpha(x, t) \equiv a(x, t), \quad \beta(x, t) \equiv b(x, t)
$$

(where $a(x, t)$ and $b(x, t)$ are the coefficients occurring in $L_{1}$ ). With this choice it is possible to derive by means of the energy integral method a priori estimates for solutions of initial value problems with a singular perturbation of hyperbolic type, provided that the coefficients a, $b, c_{1}$ and $c_{2}$ in $L_{1}$ and $L_{2}$ satisfy a so-called "timelike" condition (see DE JAGER [26]).

A generalized version of the energy integral method described above has been applied by DZAVADOV [7] to singularly perturbed initial value problems in $\mathbb{I R}^{\mathrm{n}}$. In that case the differential equation is multiplied by $L_{1}[u]$ and integrated over a suitably chosen region. For a problem in $\operatorname{IR}^{2}$ multiplication by $L_{1}[u]$ comes down to the choice $(22)$, provided that $d(x, t) \equiv 0$. 
An application of the energy integral method to singularly perturbed initialboundary value problems in $\mathbb{I R}^{\mathrm{n}}$ with a quasilinear first order operator $L_{1}$ of the type

$$
L_{1}[u]=a(\vec{x}, t) \frac{\partial u}{\partial t}+\sum_{k=1}^{n} b_{k}(\vec{x}, t) \frac{\partial u}{\partial x_{k}}+c(\vec{x}, t)|u|^{\rho} u, \quad \rho>0
$$

can be found in GENET \& MADAUNE [17].

A different form of the energy integral method, based on a multiplication of the equation by a second derivative of $u$, has been used by BLONDEL [3].

There exists a large amount of literature on singular perturbation problems. Singular perturbations of elliptic type have been studied for instance by BESJES [2], ECKHAUS [9], ECKHAUS \& DE JAGER [12], GRASMAN [20], DE GROEN [21], VAN hARTEN [23], HUET [24*], DE JAGER [25], LIONS [28], O'MALLEY [37] and VISIK \& LYUSTERNIK [49]; for singular perturbations of parabolic type we refer to BESJES [2] and LIONS [28]. Extensive bibliographies can be found in LIONS [28], NAYFEH [32] and O'MALLEY [37], [38]. Considering this large amount of literature the number of articles that deal with singular perturbations of hyperbolic type is relatively small. BLONDEL [3], [4], DZAVADOV [7], DE JAGER [26], SMITH [44], SMITH \& PALMER [46] and WEINSTEIN \& SMITH [55] have studied singularly perturbed initial value problems for linear hyperbolic differential equations, whereas singularly perturbed initial-boundary value problems have been investigated by DZAVADOV [8], GENET \& PUPION [18] and WEINSTEIN \& SMITH [55] (linear problems) and by GENET \& MADAUNE [17] and MADAUNE [30] (nonlinear problems). Singular perturbations of hyperbolic type are also discussed in the books by COLE [6], LIONS [28], ROSEAU [41] and WHITHAM [56].

We shall end this introduction with a survey of the contents of this thesis.

The first two chapters of the thesis are devoted to singularly perturbed initial value problems for ordinary differential equations of the second order, because problems of this kind can be considered as special cases of similar problems for hyperbolic equations and in our opinion the methods that will be used later on in the analysis of singularly perturbed initial value problems for hyperbolic differential equations can best be understood by applying these methods first to relatively simple problems for ordinary differential equations. 
xvi

In chapter I we deal with linear problems of the type

$$
\begin{cases}\varepsilon \frac{d^{2} u}{d t^{2}}+a(t) \frac{d u}{d t}+b(t) u=f(t), & 0<t<\infty \\ u(0 ; \varepsilon)=C_{1}, \quad u_{t}(0 ; \varepsilon)=C_{2} & \end{cases}
$$

where the coefficient $a(t)$ is strictly positive.

Applying the energy integral method we establish pointwise a priori estimates for the solution of the initial value problem and its first derivative, which are uniformly valid in any bounded segment $0 \leq t \leq T$ for $\varepsilon$ sufficiently small. Next we construct a formal approximation for the solution and use the a priori estimates to prove its correctness. It is shown that the difference between the solution and the formal approximation and also the difference between their first derivatives tend pointwise to zero as $\varepsilon$ approaches zero, uniformly in any bounded segment $0 \leq t \leq T$. We investigate also problems of the type (23) with a strictly negative coefficient a(t). It is shown that in this case the solution tends pointwise to infinity as $\varepsilon$ approaches zero.

In chapter II we deal with nonlinear problems of the type

$$
\begin{cases}\varepsilon \frac{d^{2} u}{d t^{2}}+a(t, u) \frac{d u}{d t}+b(t, u)=0, & 0<t<\infty \\ u(0 ; \varepsilon)=C_{1}, \quad u_{t}(0 ; \varepsilon)=C_{2} & \end{cases}
$$

where the coefficient $a(t, u)$ is strictly positive.

We construct a formal approximation and formulate an estimation theorem from which the existence of a solution near the formal approximation follows; the difference between this solution and the formal approximation and also the difference between their first derivatives are shown to converge pointwise to zero for $\varepsilon$ tending to zero, uniformly in any bounded segment $0 \leq t \leq T$ where the solution $w_{0}$ of the reduced problem is regular. The estimation theorem is a modified version of a similar theorem by VAN HARTEN [23] and its proof is based on a contraction mapping principle for operators in a Banach space.

In the two chapters that follow we extend the results of the chapters I and II to singularly perturbed initial value problems for hyperbolic differential equations. In chapter III we discuss linear problems of the type 


$$
\begin{cases}\varepsilon L_{2}[u]+L_{1}[u]=f(x, t), & -\infty<x<\infty, 0<t<\infty \\ u(x, 0 ; \varepsilon)=g(x), & -\infty<x<\infty \\ u_{t}(x, 0 ; \varepsilon)=h(x), & -\infty<x<\infty\end{cases}
$$

where the operators $L_{2}$ and $L_{1}$ are given by (2) and (3). The coefficient $a(x, t)$ is assumed to be strictly positive. An energy integral method is employed which yields pointwise a priori estimates for the solution of the initial value problem and its first derivatives, provided that the coefficients satisfy the so-called "timelike" condition

$$
c_{1}(x, t)<\frac{b(x, t)}{a(x, t)}<c_{2}(x, t) \text { for }-\infty<x<\infty, 0 \leq t<\infty \text {. }
$$

These a priori estimates are, for $\varepsilon$ sufficiently small, uniformly valid in any compact subset of $-\infty<\mathrm{x}<\infty, 0 \leq t<\infty$. The energy integral method that is employed is an extension of a similar method developed by DE JAGER [26] and is basically different from the methods used by other authors (cf. $[3],[4],[7],[44],[46]$ and [55]). Next we construct a formal approximation for the solution and use the a priori estimates to show that the difference between the solution and the formal approximation and also the difference between their first derivatives tend pointwise to zero as $\varepsilon$ approaches zero, uniformly in any compact subset of $-\infty<\mathrm{x}<\infty, 0 \leq t<\infty$. In chapter IV we investigate nonlinear problems of the type

$$
\left\{\begin{array}{l}
\varepsilon\left\{\frac{\partial^{2} u}{\partial t^{2}}-c^{2}(x, t) \frac{\partial^{2} u}{\partial x^{2}}\right\}+L_{1}[u]=0, \quad-\infty<x<\infty, 0<t<\infty \\
u(x, 0 ; \varepsilon)=f(x), \quad-\infty<x<\infty \\
u_{t}(x, 0 ; \varepsilon)=g(x), \quad-\infty<x<\infty
\end{array}\right.
$$

where the operator $L_{1}$ is given by $(4)$ and the functions $c(x, t)$ and $a(x, t, u)$ are strictly positive. It is shown that a formal approximation can be constructed if the solution $\mathrm{w}_{0}$ of the quasilinear reduced problem is sufficiently smooth. The existence of a solution of the full equation near this formal approximation is established by applying the estimation theorem of chapter II; the difference between this solution and the formal approximation and also the difference between their first derivatives are shown to converge pointwise to zero for $\varepsilon$ tending to zero, uniformly in compact subsets 
xvili

of $-\infty<x<\infty, 0 \leq t<\infty$ where $w_{0}$ is regular. As in chapter III the results are obtained under the condition that the coefficients satisfy a "timelike" condition. Here this condition reads

$$
-c(x, t)<\frac{b(x, t, u)}{a(x, t, u)}<c(x, t) \quad \text { for }-\infty<x, u<\infty, 0 \leq t<\infty \text {. }
$$

Generally, however, the solution $w_{0}$ of the quasilinear reduced problem does not exist in the classical sense for all $t \geq 0$. Hence we are led to the concept of "generalized solution of the reduced problem". A discussion of generalized solutions and their relevance to singular perturbations of hyperbolic type is presented.

The last two chapters of this thesis are devoted to singularly perturbed initial-boundary value problems for hyperbolic differential equations. In chapter $V$ we consider the following problem in a strip:

$$
\left\{\begin{array}{l}
q\left\{\frac{\partial^{2} u}{\partial t^{2}}-c^{2}(x, t) \frac{\partial^{2} u}{\partial x^{2}}\right\}+L_{1}[u]=f(x, t), \quad 0<x<\ell, 0<t<\infty \\
u(x, 0 ; \varepsilon)=g(x), \quad 0 \leq x \leq l \\
u_{t}(x, 0 ; \varepsilon)=h(x), \quad 0 \leq x \leq \ell \\
u(0, t ; \varepsilon)=k_{1}(t), \quad 0 \leq t<\infty \\
u(l, t ; \varepsilon)=k_{2}(t), \quad 0 \leq t<\infty
\end{array}\right.
$$

where the operator $\mathrm{L}_{1}$ is given by $(3)$, the coefficients $c(x, t)$ and $a(x, t)$ are strictly positive and the boundary functions satisfy

$$
g(0)=k_{1}(0), \quad g(\ell)=k_{2}(0)
$$

Applying a modified version of the energy integral method developed in chapter III we derive pointwise a priori estimates for the solution of this problem. We give also pointwise estimates for the first derivatives of the solution. The estimates are, for $\varepsilon$ sufficiently small, uniformly valid in any compact subset of $0 \leq \mathrm{x} \leq \ell, 0 \leq t<\infty$.

Under the additional condition $\mathrm{b}(\mathrm{x}, \mathrm{t})>0$ we construct a formal approximation for the solution. The correctness of the approximation is established by applying the a priori estimates; we show that the difference between the 
solution and the formal approximation tends pointwise to zero as $\varepsilon$ approaches zero, uniformly in any compact subset of $0 \leq x \leq l, 0 \leq t<\infty$. Here, too, the results are obtained under the restriction that the coefficients satisfy the "timelike" condition.

$$
-c(x, t)<\frac{b(x, t)}{a(x, t)}<c(x, t) \quad \text { for } 0 \leq x \leq l, 0 \leq t<\infty
$$

In chapter VI we investigate boundary value problems of the type

$$
\left\{\begin{array}{l}
\varepsilon \frac{\partial^{2} u}{\partial x \partial y}+a(x, y) \frac{\partial u}{\partial x}+b(x, y) \frac{\partial u}{\partial y}+c(x, y) u=f(x, y), \quad(x, y) \in D \\
u(x, 0 ; \varepsilon)=g(x), \quad 0 \leq x<\infty \\
u(\phi(y), y ; \varepsilon)=h(y), \quad 0 \leq y<\infty
\end{array}\right.
$$

where the region $D$ is given by

$$
D=\{(x, y) \mid \phi(y)<x<\infty, 0<y<\infty\}
$$

with $\phi(y)$ some non-decreasing function defined for $0 \leq y<\infty$ and satisfying $\phi(0)=0$. The coefficients $a(x, y)$ and $b(x, y)$ are strictly positive and the boundary functions satisfy

$$
g(0)=h(0)
$$

Note that in this problem the part of the boundary given by $y=0,0 \leq x<\infty$ coincides with a characteristic of the second order differential operator

$$
\frac{\partial^{2}}{\partial x \partial y}
$$

Problems of this kind are sometimes referred to as Goursat problems. Applying a modified version of the energy integral method developed in chapter III we establish pointwise a priori estimates for the solution of this boundary value problem which are uniformly valid in any compact subset of $\bar{D}$ for $\varepsilon$ sufficiently small. Next we construct a formal approximation for the solution and use the a priori estimates to show that the difference between the solution and the formal approximation converges pointwise to zero for $\varepsilon$ tending to zero, uniformly in any compact subset of $\bar{D}$. 



\section{NOTATIONS}

Numbers

$\mathbb{Z}:$ integers.

$\mathbb{I N}$ : positive integers.

IR: real numbers.

$\mathbb{R}^{\mathrm{n}}:=\left\{\left(\mathrm{x}_{1}, \mathrm{x}_{2}, \ldots, \mathrm{x}_{\mathrm{n}}\right) \mid \mathrm{x}_{\mathrm{k}} \in \mathbb{R} ; \mathrm{k}=1,2, \ldots, \mathrm{n}\right\}$ with $\mathrm{n} \in \mathbb{N}, \mathrm{n} \geq 2$.

$\mathbb{R}^{+}$: positive real numbers.

$\mathbb{R}^{+}$: nonnegative real numbers.

$\mathbb{C}$ : complex numbers.

$\operatorname{Re} \lambda$ : real part of $\lambda \in \mathbb{C}$.

$\operatorname{Im} \lambda$ : imaginary part of $\lambda \in \mathbb{C}$.

$\arg \lambda$ : argument of $\lambda \in \mathbb{C}, \lambda \neq 0$.

$\vec{a} \cdot \vec{b}$ : dot or scalar product of vectors $\vec{a}$ and $\vec{b}$.

$\vec{a} \times \vec{b}$ : cross or vector product of vectors $\vec{a}$ and $\vec{b}$.

$\bar{D}$ : closure of the open set $\mathrm{D} \subset \mathbb{R}^{2}$.

วD: boundary $\bar{D} \backslash D$ of the open set $D \subset \mathbb{R}^{2}$.

Functions

$u(t)$ : real-valued function of the variable $t \in \mathbb{R}$.

$u(t ; \varepsilon)$ : real-valued function of the variable $t \in \mathbb{R}$, depending on the parameter $\varepsilon \in \mathbb{R}$.

$u^{\prime}=u_{t}=\frac{d u}{d t}$ first derivative of $u(t)$ or $u(t ; \varepsilon)$.

$u^{\prime \prime}=u_{t t}=\frac{d^{2} u}{d t^{2}}$ : second derivative of $u(t)$ or $u(t ; \varepsilon)$.

$u(x, t):$ real-valued function of the variables $x, t \in \mathbb{R}$.

$u(x, t ; \varepsilon)$ : real-valued function of the variables $x, t \in \mathbb{R}$, depending on the parameter $\varepsilon \in \mathbb{R}$.

$u_{t}=\frac{\partial u}{\partial t}, u_{x}=\frac{\partial u}{\partial x}$ : first derivatives of $u(x, t)$ or $u(x, t ; \varepsilon)$.

$u_{t t}=\frac{\partial^{2} u}{\partial t^{2}}, u_{x t}=\frac{\partial^{2} u}{\partial t \partial x}, u_{x x}=\frac{\partial^{2} u}{\partial x^{2}}$ : second derivatives of $u(x, t)$ or $u(x, t ; \varepsilon)$. 
xxii

Order symbols

If $n \in \mathbb{N}$ and $u(x, t ; \varepsilon)$ is a function defined in $\Omega \subset \mathbb{R}^{2}$ then

$\mathrm{u}(\mathrm{x}, \mathrm{t} ; \varepsilon)=O\left(\varepsilon^{\mathrm{n}}\right), \varepsilon+0$ uniformly in $\Omega$ iff there exist positive constants

$\mathrm{C}$ and $\varepsilon_{0}$ such that $|u(x, t ; \varepsilon)| \leq C \varepsilon^{\mathrm{n}}$ for $(\mathrm{x}, \mathrm{t}) \in \Omega$ and $0<\varepsilon \leq \varepsilon_{0}$.

Function spaces

$\mathrm{C}^{0}[0, \mathrm{~T}]:$ space of real-valued, continuous functions defined in $[0, \mathrm{~T}] \subset \mathbb{R}$.

$C^{k}[0, T], k \in \mathbb{N}$ : space of real-valued, $k$ times continuously differentiable functions defined in $[0, T] \subset \mathbb{R}$.

$C^{\infty}[0, \mathrm{~T}]:={ }_{k=0}^{\infty} \mathrm{n}_{0} \mathrm{C}^{\mathrm{k}}[0, \mathrm{~T}]$.

$\mathrm{C}^{0}(\Omega), \mathrm{C}^{\mathrm{k}}(\Omega)$ and $\mathrm{C}^{\infty}(\Omega)$ denote similar spaces of real-valued functions defined in $\Omega \subset \mathbb{R}^{2}$.

Norms

For functions of the variable $t$ defined in $0 \leq t \leq T$ :

$$
\begin{aligned}
& |u|_{0}=\sup _{[0, T]}|u|, \quad|u|_{1}^{(\varepsilon)}=\sup _{[0, T]}|u|+\varepsilon^{\frac{1}{2}} \sup _{[0, T]}\left|u_{t}\right| \\
& \|u\|_{[0, T]}=\left[\int_{0}^{T} u^{2} d t\right]^{\frac{1}{2}}, \quad\|u\|_{1,[0, T]}=\int_{0}^{T}|u| d t
\end{aligned}
$$

For functions of the variables $x$ and $t$ defined in a compact subset $\Omega \subset \mathbb{I}^{2}$ :

$$
\begin{aligned}
& |u|_{0}=\sup _{\Omega}|u|, \quad|u|_{1}^{(\varepsilon)}=\sup _{\Omega}|u|+\varepsilon^{\frac{3}{4}} \sup _{\Omega}\left|u_{x}\right|+\varepsilon^{\frac{3}{4}} \sup _{\Omega}\left|u_{t}\right| \\
& \|u\|_{\Omega}=\left[\iint_{\Omega} u^{2} d x d t\right]^{\frac{1}{2}}
\end{aligned}
$$

Generic constants

Sometimes $C$ and $\varepsilon_{0}$ denote generic constants of which the value may be different on each appearance. 


\section{CHAPTER I}

INITIAL VALUE PROBLEMS FOR LINEAR

ORDINARY DIFFERENTIAL EQUATIONS

In this chapter we shall discuss the initial value problem (singular perturbation problem)

$$
\left\{\begin{array}{l}
\varepsilon \frac{d^{2} u}{d t^{2}}+a(t ; \varepsilon) \frac{d u}{d t}+b(t ; \varepsilon) u=f(t ; \varepsilon), \quad 0<t<\infty \\
u(0 ; \varepsilon)=C_{1}(\varepsilon), \quad u_{t}(0 ; \varepsilon)=C_{2}(\varepsilon)
\end{array}\right.
$$

where $\varepsilon$ is a small positive parameter, i.e. $0<\varepsilon<1$.

The functions $a, b$ and $f$ depend on the variable $t$ and on the parameter $\varepsilon$. The initial data $C_{1}(\varepsilon)$ and $C_{2}(\varepsilon)$ are constants depending on $\varepsilon$.

It will be assumed that the coefficient $a(t ; \varepsilon)$ is either strictly positive

$$
a(t ; \varepsilon) \geq a_{0}>0 \quad \text { for } 0 \leq t \leq T
$$

or strictly negative

$$
a(t ; \varepsilon) \leq-a_{0}<0 \quad \text { for } 0 \leq t \leq T
$$

valid for each constant $T>0$ provided that $\varepsilon$ is sufficiently small, say $0<\varepsilon \leq \varepsilon_{0}$ with $\varepsilon_{0}$ depending on $T$. Here $a_{0}$ is some positive constant depending on $T$ and $\varepsilon_{0}$ but independent of $\varepsilon$.

There exists a large amount of literature on singularly perturbed initial value problems for ordinary differential equations. Most authors investigate these problems by writing them as a system of first order equations, see for instance HOPPENSTEADT [24], O'MALLEY [38, chapter 4], PONZO \& WAX [39], VASIL'EVA [47], VASIL'EVA \& VOLOSOV [48] and WASOW [52, chapter 10]. The problems considered by these authors are generally nonlinear. 
Singularly perturbed initial value problems in the form of a single differential equation of higher order are less frequently encountered in literature. We mention here the work of GRADSTEIN [19], O'MALLEY [37], SMITH [45], VOLOSOV [50], [51] and WEINSTEIN \& SMITH [54].

In spite of this wide variety of literature we have thought it appropriate to devote the first two chapters of this thesis to singularly perturbed initial value problems for linear and nonlinear ordinary differential equations of order two. Our considerations are that initial value problems for hyperbolic differential equations (which form the main subject of our investigations) can be solved essentially in the same way as initial value problems for ordinary differential equations, so the methods that will be used in the analysis of linear and nonlinear hyperbolic equations (chapters III and IV) can best be understood by applying them first to relatively simple problems which are governed by linear and nonlinear ordinary differential equations (chapters I and II).

In the sections 1 and 2 of this chapter we shall study problem (1) with a strictly positive coefficient $a(t ; \varepsilon)$. We shall derive a priori estimates for the solution $u$ of (1) (section 1) and construct an asymptotic expansion into powers of $\varepsilon$ for this solution (section 2).

In section 3 we shall discuss problem (1) in case the coefficient $a(t ; \varepsilon)$ is strictly negative.

\section{A priori estimates}

We consider the initial value problem (1) under the following conditions:

(2)

for each positive constant $T$ there exists a positive constant $\varepsilon_{0}$ (depending on $\mathrm{T}$ ) such that

(i) $\quad a(t ; \varepsilon) \in C^{1}[0, T], \quad b(t ; \varepsilon) \in C^{1}[0, T], \quad f(t ; \varepsilon) \in C^{0}[0, T]$ valid for every value of $\varepsilon$ satisfying $0<\varepsilon \leq \varepsilon_{0}$

(ii) the functions $a(t ; \varepsilon), b(t ; \varepsilon)$ and their first derivatives are uniformly bounded in $0 \leq t \leq T$ for $0<\varepsilon \leq \varepsilon_{0}$, i.e. there exist positive constants $A$ and $B$ (depending on $T$ and $\varepsilon_{0}$ but independent of $\varepsilon)$ such that for all $t \in[0, T]$ and all $\varepsilon \in\left(0, \varepsilon_{0}\right]$ 
(2)

(iii) $a(t ; \varepsilon) \geq a_{0}>0$

valid for all $t \in[0, T]$ and all $\varepsilon \in\left(0, \varepsilon_{0}\right]$, where $a_{0}$ is some constant depending on $T$ and $\varepsilon_{0}$ but independent of $\varepsilon$.

For the moment it will be assumed that the initial conditions are homogeneous, so

$$
C_{1}(\varepsilon)=C_{2}(\varepsilon)=0
$$

In order to derive an estimate for the solution $u(t ; \varepsilon)$ of $(1)$ in an interval $0 \leq t \leq T$ we multiply the differential equation (1) by $2 u$. This yields

$$
\frac{d}{d t}\left(a u^{2}+2 \varepsilon u u_{t}\right)+\left(2 b-a_{t}\right) u^{2}-2 \varepsilon u_{t}^{2}=2 f u
$$

valid for $0 \leq t \leq T$ and $0<\varepsilon \leq \varepsilon_{0}$, where arguments have been omitted for simplicity.

Similarly, multiplication of (1) by $2 a_{t}$ leads to

$$
\frac{d}{d t}\left(\varepsilon a u_{t}^{2}\right)-\varepsilon a_{t} u_{t}^{2}+2 a^{2} u_{t}^{2}+2 a b u u_{t}=2 a f u_{t}
$$

valid for $0 \leq t \leq T$ and $0<\varepsilon \leq \varepsilon_{0}$.

Adding (4) and (5) and using the inequalities

$$
\left\{\begin{array}{l}
-2 a^{2} u_{t}^{2}-2 a b u u_{t}+2 a f u_{t} \leq b^{2} u^{2}+f^{2} \\
2 f u \leq u^{2}+f^{2}
\end{array}\right.
$$

we obtain

$$
\frac{d}{d t}\left(a u^{2}+2 \varepsilon u u_{t}+\varepsilon a u_{t}^{2}\right) \leq\left(1+a_{t}+b^{2}-2 b\right) u^{2}+\varepsilon\left(2+a_{t}\right) u_{t}^{2}+2 f^{2}
$$

valid for $0 \leq t \leq T$ and $0<\varepsilon \leq \varepsilon_{0}$. 
From the positiveness of $a(t ; \varepsilon)$ it follows that for $0 \leq t \leq T$ and $\varepsilon$ sufficiently small, say $0<\varepsilon \leq \varepsilon_{0}$ with $\varepsilon_{0}$ a generic constant

$$
\begin{aligned}
a u^{2}+2 \varepsilon u u_{t}+\varepsilon a u_{t}^{2} & \geq a_{0} u^{2}+2 \varepsilon u u_{t}+\varepsilon a_{0} u_{t}^{2} \\
& \geq\left(a_{0}-\varepsilon^{\frac{1}{2}}\right) u^{2}+\varepsilon\left(a_{0}-\varepsilon^{\frac{1}{2}}\right) u_{t}^{2} \\
& \geq m\left(u^{2}+\varepsilon u_{t}^{2}\right)
\end{aligned}
$$

where the positive constant $\mathrm{m}$ (independent of $\varepsilon$ ) may be defined by

$$
\mathrm{m}=\frac{1}{2} \mathrm{a}_{0}
$$

So integrating (6) with respect to $t$ and using this inequality as well as (3) we obtain

$$
m\left(u^{2}+\varepsilon u_{t}^{2}\right) \leq M \int_{0}^{t}\left(u^{2}+\varepsilon u_{t}^{2}\right) d \tau+M\|f\|_{[0, T]}^{2}
$$

or

$$
\left(u^{2}+\varepsilon u_{t}^{2}\right)-\frac{M}{m} \int_{0}^{t}\left(u^{2}+\varepsilon u_{t}^{2}\right) d \tau \leq \frac{M}{m}\|f\|_{[0, T]}^{2}
$$

valid for $0 \leq t \leq T$ and $0<\varepsilon \leq \varepsilon_{0}$, where the positive constant $M$ (independent of $\varepsilon$ ) is given by

$$
M=\max \left(2+A, 1+A+B^{2}+2 B\right)
$$

and $\|\cdot\|_{[0, T]}$ denotes the $\mathrm{L}_{2}$-norm.

Using GRONWALL's lemma (see for instance RUBINSTEIN $[43$, p.26]) we obtain from (7)

$$
\begin{aligned}
u^{2}+\varepsilon u_{t}^{2} & \leq \frac{M}{m}\|f\|_{[0, T]}^{2} \exp \left(\frac{M t}{m}\right) \\
& \leq \frac{M}{m}\|f\|_{[0, T]}^{2} \exp \left(\frac{M T}{m}\right)
\end{aligned}
$$

and hence 
(8)

$$
\left\{\begin{array}{l}
|u(t ; \varepsilon)| \leq C(T)\|f\|_{[0, T]} \\
\left|\frac{d u}{d t}(t ; \varepsilon)\right| \leq C(T) \varepsilon^{-\frac{1}{2}\|f\|_{[0, T]}}
\end{array}\right.
$$

valid for $0 \leq t \leq T$ and $0<\varepsilon \leq \varepsilon_{0}$, where the positive constant $C(T)$, depending on $T$ but independent of $\varepsilon$, is given by

$$
C(T)=\left(\frac{M}{m}\right)^{\frac{1}{2}} \exp \left(\frac{M T}{2 m}\right)
$$

Note that so far we did not use the differentiability of the coefficient $b(t ; \varepsilon)$ required in $(2, i$ and $i i)$.

Now that we have established estimates for the solution of the homogeneous initial value problem (1) it is not difficult to obtain estimates for the inhomogeneous problem. We put

$$
u(t ; \varepsilon)=w(t ; \varepsilon)+p \exp \left\{-\int_{0}^{t} \frac{b(\tau ; \varepsilon)}{a(\tau ; \varepsilon)} d \tau\right\}-\varepsilon q \exp \left\{-\frac{a(0 ; \varepsilon) t}{\varepsilon}\right\}
$$

where the $\varepsilon$-dependent constants $p$ and $q$ are defined by

$$
\left\{\begin{array}{l}
p=\frac{a(0 ; \varepsilon)\left\{a(0 ; \varepsilon) C_{1}(\varepsilon)+\varepsilon C_{2}(\varepsilon)\right\}}{a^{2}(0 ; \varepsilon)-\varepsilon b(0 ; \varepsilon)}, \\
q=\frac{b(0 ; \varepsilon) C_{1}(\varepsilon)+a(0 ; \varepsilon) C_{2}(\varepsilon)}{a^{2}(0 ; \varepsilon)-\varepsilon b(0 ; \varepsilon)} .
\end{array}\right.
$$

Substitution of (9) into (1) yields

$$
\left\{\begin{array}{l}
\varepsilon \frac{d^{2} w}{d t^{2}}+a(t ; \varepsilon) \frac{d w}{d t}+b(t ; \varepsilon) w=F(t ; \varepsilon), \quad 0<t<\infty \\
w(0 ; \varepsilon)=w_{t}(0 ; \varepsilon)=0
\end{array}\right.
$$

with

$$
\begin{aligned}
F(t ; \varepsilon)=f(t ; \varepsilon) & -\varepsilon p \frac{d^{2}}{d t^{2}} \exp \left\{-\int_{0}^{t} \frac{b(\tau ; \varepsilon)}{a(\tau ; \varepsilon)} d \tau\right\} \\
& -q a(0 ; \varepsilon)\{a(t ; \varepsilon)-a(0 ; \varepsilon)\} \exp \left\{-\frac{a(0 ; \varepsilon) t}{\varepsilon}\right\}+
\end{aligned}
$$




$$
+\varepsilon q b(t ; \varepsilon) \exp \left\{-\frac{a(0 ; \varepsilon) t}{\varepsilon}\right\}
$$

Using the positiveness of $a(t ; \varepsilon)$ it is easily verified that there exists a generic constant $C(T)$, depending on $T$ but independent of $\varepsilon$, such that

$$
\|F\|_{[0, T]} \leq C(T)\left\{\|_{[0, T]}+\varepsilon\left|C_{1}(\varepsilon)\right|+\varepsilon^{3 / 2}\left|C_{2}(\varepsilon)\right|\right\}
$$

So applying the estimates (8) to the function $w$ and using (9) as well as the positiveness of $a(t ; \varepsilon)$ we obtain

$$
\left\{\begin{array}{l}
|u(t ; \varepsilon)| \leq C(T)\left\{\left\|_{f}\right\|_{[0, T]}+\left|C_{1}(\varepsilon)\right|+\varepsilon\left|C_{2}(\varepsilon)\right|\right\} \\
\left|\frac{d u}{d t}(t ; \varepsilon)\right| \leq C(T)\left\{\varepsilon^{-\frac{1}{2}}\left\|_{f}\right\|_{[0, T]}+\left|C_{1}(\varepsilon)\right|+\left|C_{2}(\varepsilon)\right|\right\}
\end{array}\right.
$$

valid for $0 \leq t \leq T$ and $0<\varepsilon \leq \varepsilon_{0}$, with $C(T)$ a generic constant.

We summarize the results in

THEOREM 1. Let $\mathrm{u}(t ; \varepsilon)$ be the solution of the initial value problem (1) under the conditions (2). Then we have in every interval $0 \leq t \leq \mathrm{T}$ the estimates

$$
\left\{\begin{array}{l}
\sup _{[0, T]}|u(t ; \varepsilon)| \leq C(T)\left\{\|f\|_{[0, T]}+\left|C_{1}(\varepsilon)\right|+\varepsilon\left|C_{2}(\varepsilon)\right|\right\} \\
\sup _{[0, T]}\left|\frac{d u}{d t}(t ; \varepsilon)\right| \leq C(T)\left\{\varepsilon^{-\frac{1}{2} \|} \|_{[0, T]}+\left|C_{1}(\varepsilon)\right|+\left|C_{2}(\varepsilon)\right|\right\}
\end{array}\right.
$$

valid for all values of $\varepsilon$ satisfying $0<\varepsilon \leq \varepsilon_{0}$, where $\varepsilon_{0}$ is a constant depending on $\mathrm{T}$ and $\mathrm{C}(\mathrm{T})$ is a positive constant depending on $\mathrm{T}$ but independent of $\varepsilon$.

\section{REMARKS.}

1. The differentiability of the coefficient $b(t ; \varepsilon)$ can be disposed of in case $C_{1}(\varepsilon)=C_{2}(\varepsilon)=0$ (see formula (8)).

2. An estimate for the second derivative $\frac{d^{2} u}{d t^{2}}$ follows by substitution of (10a) and (10b) into the differential equation (1). 


\section{Asymptotic expansions}

As an application of the a priori estimates that have been established in the preceding section we shall now construct an asymptotic expansion into powers of $\varepsilon$ for the solution $u(t ; \varepsilon)$ of the initial value problem (1). In order not to complicate the calculations we shall assume that the functions $a, b$ and $f$ as well as the initial data $c_{1}$ and $c_{2}$ are independent of the parameter $\varepsilon$. Furthermore we shall assume that $a, b$ and $f$ are $c^{\infty}$-functions. So we consider the initial value problem

$$
\left\{\begin{array}{l}
\varepsilon \frac{d^{2} u}{d t^{2}}+a(t) \frac{d u}{d t}+b(t) u=f(t), \quad 0<t<\infty \\
u(0 ; \varepsilon)=C_{1}, \quad u_{t}(0 ; \varepsilon)=C_{2}
\end{array}\right.
$$

with $0<\varepsilon<1$ and

$$
\left\{\begin{aligned}
a(t) \in C^{\infty} \overline{\left(\mathbb{I R}^{+}\right)}, \quad b(t) \in C^{\infty} \overline{\left(\mathbb{I R}^{+}\right)}, \quad f(t) \in C^{\infty} \overline{\left(\mathbb{R}^{+}\right)} \\
a(t)>0 \text { for } 0 \leq t<\infty .
\end{aligned}\right.
$$

Expanding the functions $a(t)$ and $b(t)$ into a Taylor series we obtain for any $\mathrm{n} \in \mathbb{I N} \cup\{0\}$

$$
\left\{\begin{array}{l}
a(t)=\sum_{k=0}^{n} a_{k} t^{k}+a_{n+1}(t) t^{n+1} \\
b(t)=\sum_{k=0}^{n} b_{k} t^{k}+b_{n+1}(t) t^{n+1}
\end{array}\right.
$$

with $a_{0}=a(0), b_{0}=b(0)$ etc.

We define the differential operator $L^{(\varepsilon)}$ by

$$
L^{(\varepsilon)}[u]=\varepsilon \frac{d^{2} u}{d t^{2}}+a(t) \frac{d u}{d t}+b(t) u .
$$

Putting $\tau=\frac{t}{\varepsilon}$ and expanding $L^{(\varepsilon)}$ into powers of $\varepsilon$ we obtain

$$
\begin{aligned}
L^{(\varepsilon)}[u]=\varepsilon^{-1} \frac{d^{2} u}{d \tau^{2}} & +\varepsilon^{-1}\left\{\sum_{k=0}^{n} a_{k} \varepsilon^{k} \tau^{k}+a_{n+1}(\varepsilon \tau) \varepsilon^{n+1} \tau^{n+1}\right\} \frac{d u}{d \tau} \\
& +\sum_{k=0}^{n} b_{k} \varepsilon^{k} \tau^{k} u+b_{n+1}(\varepsilon \tau) \varepsilon^{n+1} \tau^{n+1} u .
\end{aligned}
$$


We construct now a formal approximation $\tilde{u}(t ; \varepsilon)$ of $u(t ; \varepsilon)$ in the form

$$
\tilde{u}(t ; \varepsilon)=\sum_{k=0}^{n} \varepsilon^{k} w_{k}(t)+\sum_{k=0}^{n} \varepsilon^{k+1} v_{k}(\tau)
$$

where the functions $w_{k}$ and $v_{k}(k=0,1, \ldots, n)$ have to be determined. A calculation shows that

$$
\begin{aligned}
L^{(\varepsilon)}[\tilde{u}]= & L^{(\varepsilon)}\left[\sum_{k=0}^{n} \varepsilon^{k} w_{k}\right]+L^{(\varepsilon)}\left[\sum_{k=0}^{n} \varepsilon^{k+1} v_{k}\right] \\
= & \left\{a(t) \frac{d w_{0}}{d t}+b(t) w_{0}\right\}+\left\{\frac{d^{2} v_{0}}{d \tau^{2}}+a(0) \frac{d v_{0}}{d \tau}\right\} \\
& +\sum_{k=1}^{n} \varepsilon^{k}\left\{a(t) \frac{d w_{k}}{d t}+b(t) w_{k}+\frac{d^{2} w_{k-1}}{d t^{2}}\right\} \\
& +\sum_{k=1}^{n} \varepsilon^{k}\left\{\frac{d^{2} v_{k}}{d \tau^{2}}+a(0) \frac{d v_{k}}{d \tau}+\sum_{j=1}^{k} a_{j} \tau^{j} \frac{d v_{k-j}}{d \tau}\right. \\
& \left.+\sum_{j=0}^{k-1} b_{j} \tau^{j} v_{k-j-1}\right\}+R(t ; \varepsilon)
\end{aligned}
$$

where the function $R(t ; \varepsilon)$ is defined by

$$
\begin{aligned}
R(t ; \varepsilon)= & \varepsilon^{n+1} \frac{d^{2} w_{n}}{d t^{2}}+\sum_{k=n+1}^{2 n} \varepsilon^{k}\left(\sum_{j=k-n}^{n} a_{j} \tau^{j} \frac{d v_{k-j}}{d \tau}\right) \\
& +a_{n+1}(t) \varepsilon^{n+1} \tau^{n+1} \sum_{k=0}^{n} \varepsilon^{k} \frac{d v_{k}}{d \tau}+\sum_{k=n}^{2 n} \varepsilon^{k+1}\left(\sum_{j=k-n}^{n} b_{j} \tau^{j} v_{k-j}\right) \\
& +b_{n+1}(t) \varepsilon^{n+1} \tau^{n+1} \sum_{k=0}^{n} \varepsilon^{k+1} v_{k} .
\end{aligned}
$$

N.B. Empty sums, i.e. sums of which the upper limit of summation is less than the lower limit, are to be interpreted as zero.

Putting $t=0$ in (13) we get 
(15)

$$
\left\{\begin{array}{l}
\tilde{u}(0 ; \varepsilon)=w_{0}(0)+\sum_{k=1}^{n} \varepsilon^{k}\left\{w_{k}(0)+v_{k-1}(0)\right\}+\varepsilon^{n+1} v_{n}(0) \\
\tilde{u}_{t}(0 ; \varepsilon)=\sum_{k=0}^{n} \varepsilon^{k}\left\{\frac{d w_{k}}{d t}(0)+\frac{d v_{k}}{d \tau}(0)\right\} .
\end{array}\right.
$$

The formal approximation $\tilde{u}$ of the solution $u$ of (11) is now obtained by solving the following set of initial value problems

$$
\begin{aligned}
& \left\{\begin{array}{l}
a(t) \frac{d w_{0}}{d t}+b(t) w_{0}=f(t), \quad 0<t<\infty \\
w_{0}(0)=c_{1}
\end{array}\right. \\
& \left\{\begin{array}{l}
\frac{d^{2} v_{0}}{d \tau^{2}}+a(0) \frac{d v_{0}}{d \tau}=0, \quad 0<\tau<\infty \\
\frac{d v_{0}}{d \tau}(0)=c_{2}-\frac{d w_{0}}{d t}(0) \\
\lim _{\tau \rightarrow \infty} v_{0}(\tau)=0
\end{array}\right.
\end{aligned}
$$

and for $\mathrm{k}=1,2, \ldots, \mathrm{n}$

$$
\begin{aligned}
& \left\{\begin{array}{l}
a(t) \frac{d w_{k}}{d t}+b(t) w_{k}=-\frac{d^{2} w_{k-1}}{d t^{2}}, \quad 0<t<\infty \\
w_{k}(0)=-v_{k-1}(0)
\end{array}\right. \\
& \left\{\begin{array}{l}
\frac{d^{2} v_{k}}{d \tau^{2}}+a(0) \frac{d v_{k}}{d \tau}=-\sum_{j=1}^{k} a_{j} \tau^{j} \frac{d v_{k-j}}{d \tau}-\sum_{j=0}^{k-1} b_{j} \tau^{j} v_{k-j-1}, \quad 0<\tau<\infty \\
\frac{d v_{k}}{d \tau}(0)=-\frac{d w_{k}}{d t}(0) \\
\lim _{\tau \rightarrow \infty} v_{k}(\tau)=0 .
\end{array}\right.
\end{aligned}
$$

It is easily established that the functions $v_{k}(\tau)$, being of the form

$$
\mathrm{v}_{\mathrm{k}}(\tau)=\mathrm{P}_{\mathrm{k}}(\tau) \exp \{-\mathrm{a}(0) \tau\}
$$


with $P_{k}(\tau)$ a polynomial in $\tau$, are typical boundary layer functions and also that (14) and (15) can be written as

$$
\left\{\begin{array}{l}
\mathrm{L}^{(\varepsilon)}[\tilde{u}]=f(t)+R(t ; \varepsilon), \quad 0<t<\infty \\
\tilde{u}(0 ; \varepsilon)=C_{1}+\varepsilon^{n+1} v_{n}(0) \\
\tilde{u}_{t}(0 ; \varepsilon)=C_{2} .
\end{array}\right.
$$

Finally it is easily verified that for any nonnegative number $T$

$$
R(t ; \varepsilon)=O\left(\varepsilon^{n+1}\right), \quad \varepsilon \downarrow 0
$$

uniformly valid in $0 \leq t \leq T$. Hence the function $\tilde{u}$ provides a formal approximation (up to order $\varepsilon^{n+1}$ ) for the solution $u$ of problem (11).

Introducing the remainder term $z(t ; \varepsilon)$ by

$$
z(t ; \varepsilon)=u(t ; \varepsilon)-\tilde{u}(t ; \varepsilon)
$$

it follows from (11) and (21) that

$$
\left\{\begin{array}{l}
\mathrm{L}^{(\varepsilon)}[\mathrm{z}]=-\mathrm{R}(t ; \varepsilon), \quad 0<t<\infty \\
\mathrm{z}(0 ; \varepsilon)=-\varepsilon^{\mathrm{n}+1} \mathrm{v}_{\mathrm{n}}(0) \\
z_{t}(0 ; \varepsilon)=0
\end{array}\right.
$$

so we obtain from theorem 1

$$
\begin{cases}z(t ; \varepsilon)=0\left(\varepsilon^{n+1}\right), & \varepsilon+0 \\ \frac{d z}{d t}(t ; \varepsilon)=0\left(\varepsilon^{n+\frac{1}{2}}\right), & \varepsilon+0\end{cases}
$$

uniformly valid in $0 \leq t \leq T$.

Since $\varepsilon^{n+1} v_{n}(\tau)$ is uniformly of order $\varepsilon^{n+1}$ in $0 \leq \tau<\infty$ it follows from (13), (22) and (23) that 


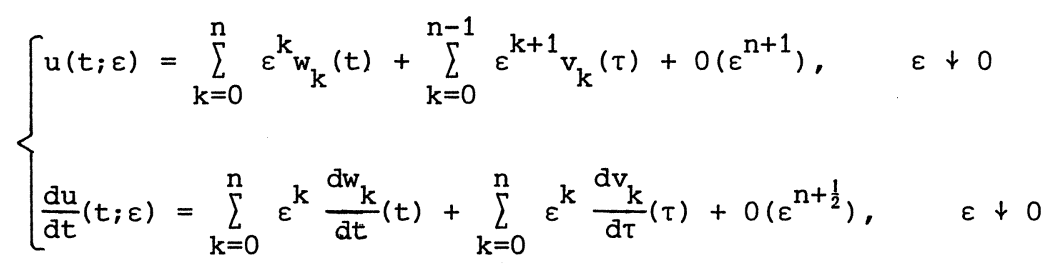

uniformly valid in $0 \leq t \leq T$.

The accuracy in the expansion for $\frac{d u}{d t}$ can be improved by applying the formula with $\mathrm{n}$ replaced by $\mathrm{n}+1$ and using the fact that the terms

$$
\varepsilon^{n+1} \frac{d w_{n+1}}{d t}(t) \quad \text { and } \quad \varepsilon^{n+1} \frac{d v_{n+1}}{d \tau}(\tau)
$$

are uniformly of order $\varepsilon^{n+1}$. In this way we obtain

THEOREM 2. Let $u(t ; \varepsilon)$ be the solution of the initial value problem (11) under the conditions (12). Then we have for any $\mathrm{n} \in \mathbb{I N} \cup\{0\}$

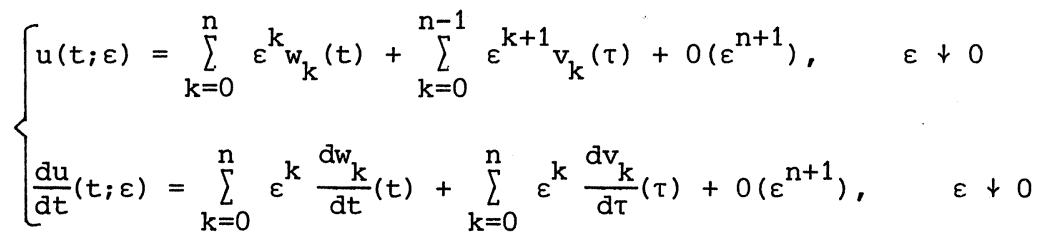

uniformly valid in every bounded interval $0 \leq t \leq T$, where $\tau=\frac{t}{\varepsilon}$ and the functions $\mathrm{w}_{\mathrm{k}}$ and $\mathrm{v}_{\mathrm{k}}(\mathrm{k}=0,1, \ldots, \mathrm{n})$ follow successively from (16), (17), (18) and (19).

In case $\mathrm{n}=0$ empty sums are to be interpreted as zero.

COROLLARY 1. It follows from (24) that first approximations $(\mathrm{n}=0)$ for the solution $\mathrm{u}$ of (11) and its derivative are given by

$$
\left\{\begin{array}{l}
u(t ; \varepsilon)=w_{0}(t)+0(\varepsilon), \quad \varepsilon+0 \\
\frac{d u}{d t}(t ; \varepsilon)=\frac{d w_{0}}{d t}(t)+\left\{c_{2}-\frac{d w_{0}}{d t}(0)\right\} \exp \left[-a(0) \frac{t}{\varepsilon}\right]+0(\varepsilon), \quad \varepsilon+0
\end{array}\right.
$$

uniformly valid in every bounded interval $0 \leq t \leq T$, where $\mathrm{w}_{0}$ is the solution of the so-called reduced differential equation (16) which follows from (11) by putting $\varepsilon=0$. 
COROLLARY 2. It follows from (24) and (20) that for any $\mathrm{n} \in \mathbb{I N} \cup\{0\}$

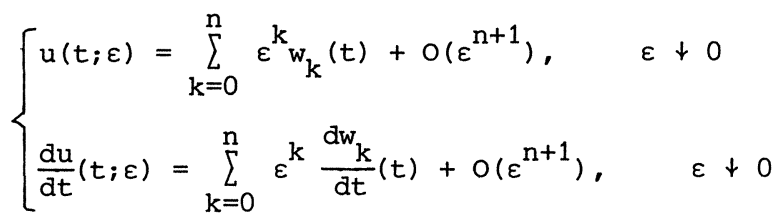

uniformly valid in every bounded interval $0<\delta \leq t \leq T$, where $\delta$ is an arbitrarily small positive constant independent of $\varepsilon$.

REMARK. If the condition of infinite smoothness of the functions $a, b$ and $\mathrm{f}$ is weakened, then the expansions (24) are valid for all $\mathrm{n} \in \mathbb{I} \cup\{0\}$ with $\mathrm{n} \leq \mathrm{n}_{0}$, the bound $\mathrm{n}_{0}$ being determined by the differentiability of the functions $a, b$ and $f$.

\section{The case $a(t ; \varepsilon) \leq-a_{0} \leq 0$}

In this section we shall study problem (1) with a strictly negative coefficient $a(t ; \varepsilon)$. In order not to complicate the calculations it will be assumed that the functions $a, b$ and $f$ are independent of $\varepsilon$; without loss of generality it will further be assumed that $C_{1}(\varepsilon)=C_{2}(\varepsilon)=0$. Thus we consider the initial value problem

$$
\left\{\begin{array}{l}
\varepsilon \frac{d^{2} u}{d t^{2}}+a(t) \frac{d u}{d t}+b(t) u=f(t), \quad 0<t<\infty \\
u(0 ; \varepsilon)=u_{t}(0 ; \varepsilon)=0
\end{array}\right.
$$

with $0<\varepsilon<1$ and

$$
\left\{\begin{array}{c}
a(t) \in C^{2}\left(\overline{\mathbb{I R}^{+}}\right), \quad b(t) \in \mathrm{C}^{1} \overline{\left(\mathbb{I R}^{+}\right)}, \mathrm{f}(t) \in \mathrm{C}^{1} \overline{\left(\mathbb{I R}^{+}\right)} \\
a(t)<0 \text { for } 0 \leq t<\infty .
\end{array}\right.
$$

Without loss of generality we may assume that there exists no interval $\left[0, t_{0}\right)$ with the property that $f(t) \equiv 0$ for $0 \leq t<t_{0}$. Otherwise we could take the largest interval with this property, say $\left[0, t_{0}^{*}\right)$, and formulate a similar initial value problem for $u$ in $t_{0}^{*}<t<\infty$ with horiogeneous initial conditions at $t=t_{0}^{*}$, the solution in $\left[0, t_{0}^{*}\right)$ being given by $u(t ; \varepsilon) \equiv 0$. 
In the preceding section we have shown that the solution $u(t ; \varepsilon)$ of problem (25) with a positive coefficient a(t) satisfies

$$
\lim _{\varepsilon \downarrow 0} u(t ; \varepsilon)=w(t)
$$

uniformly in $0 \leq t \leq T$, where $w$ is the solution of the reduced problem

$$
\left\{\begin{array}{l}
a(t) \frac{d w}{d t}+b(t) w=f(t), \quad 0<t<\infty \\
w(0)=0 .
\end{array}\right.
$$

It will turn out that the solution $u(t ; \varepsilon)$ of problem (25) with a negative coefficient $a(t)$ behaves quite differently. We shall show that for every value of $t$ with $t \geq \delta>0$ ( $\delta$ arbitrarily small but independent of $\varepsilon$ ) the solution $u(t ; \varepsilon)$ (and hence also the difference $u(t ; \varepsilon)-w(t)$ ) increases exponentially as $\varepsilon$ tends to zero.

In order to analyse the solution $u$ of (25) we put

$$
u(t ; \varepsilon)=w(t)+u(t ; \varepsilon) \exp \left\{-\frac{1}{\varepsilon} \int_{0}^{t} a(\tau) d \tau\right\}
$$

where $\mathrm{w}$ is the solution of the reduced problem (27) which is given by

$$
w(t)=\int_{0}^{t} \frac{f(\tau)}{a(\tau)} \exp \left\{-\int_{\tau}^{t} \frac{b(\xi)}{a(\xi)} d \xi\right\} d \tau \text {. }
$$

Then we obtain for $U$ the initial value problem

$$
\left\{\begin{array}{l}
\varepsilon \frac{d^{2} U}{d t^{2}}-a(t) \frac{d U}{d t}+\left\{b(t)-a^{\prime}(t)\right\} U=\varepsilon \Psi_{1}(t) \exp \left\{\frac{1}{\varepsilon} \int_{0}^{t} a(\tau) d \tau\right\}, \quad 0<t<\infty \\
U(0 ; \varepsilon)=0, \quad U_{t}(0 ; \varepsilon)=-\frac{f(0)}{a(0)}
\end{array}\right.
$$

with

$$
\Psi_{1}=-\frac{d}{d t} \frac{f}{a}+\frac{b f}{a^{2}}+\left(\frac{d}{d t} \frac{b}{a}-\frac{b^{2}}{a^{2}}\right) w,
$$

the prime denoting differentiation with respect to $t$. Defining the function $v(t ; \varepsilon)$ by 


$$
v(t ; \varepsilon)=-\int_{0}^{t} \frac{f(\tau)}{a(\tau)} \exp \left[\int_{\tau}^{t}\left\{\frac{b(\xi)-a^{\prime}(\xi)}{a(\xi)}\right\} d \xi+\frac{1}{\varepsilon} \int_{0}^{\tau} a(\xi) d \xi\right] d \tau
$$

$$
=-\frac{1}{a(t)} \int_{0}^{t} f(\tau) \exp \left\{\int_{\tau}^{t} \frac{b(\xi)}{a(\xi)} d \xi+\frac{1}{\varepsilon} \int_{0}^{\tau} a(\xi) d \xi\right\} d \tau
$$

it is easily established that $\mathrm{v}$ satisfies the initial value problem

$$
\left\{\begin{array}{l}
\varepsilon \frac{d^{2} v}{d t^{2}}-a(t) \frac{d v}{d t}+\left\{b(t)-a^{\prime}(t)\right\} v=-\varepsilon \Phi(t) v(t ; \varepsilon) \\
+\varepsilon \Psi_{2}(t) \exp \left\{\frac{1}{\varepsilon} \int_{0}^{t} a(\tau) d \tau\right\} \\
v(0 ; \varepsilon)=0, \quad v_{t}(0 ; \varepsilon)=-\frac{f(0)}{a(0)}
\end{array}\right.
$$

where

$$
\left\{\begin{array}{l}
\Phi=-\frac{d}{d t}\left(\frac{b-a^{\prime}}{a}\right)-\left(\frac{b-a^{\prime}}{a}\right)^{2} \\
\Psi_{2}=-\frac{d}{d t} \frac{f}{a}+\left(\frac{a^{\prime}-b}{a^{2}}\right) f
\end{array}\right.
$$

So putting

$$
U(t ; \varepsilon)=v(t ; \varepsilon)+z(t ; \varepsilon)
$$

we obtain from (30) and (32)

$$
\left\{\begin{array}{l}
\varepsilon \frac{d^{2} z}{d t^{2}}-a(t) \frac{d z}{d t}+\left\{b(t)-a^{\prime}(t)\right\} z=F(t ; \varepsilon), \quad 0<t<\infty \\
z(0 ; \varepsilon)=z_{t}(0 ; \varepsilon)=0
\end{array}\right.
$$

with

$$
\left\{\begin{array}{l}
F(t ; \varepsilon)=\varepsilon \Phi(t) v(t ; \varepsilon)+\varepsilon \Psi(t) \exp \left\{\frac{1}{\varepsilon} \int_{0}^{t} a(\tau) d \tau\right\} \\
\Psi=\Psi_{1}-\Psi_{2}=\left(\frac{2 b-a^{\prime}}{a^{2}}\right) f+\left(\frac{d}{d t} \frac{b}{a}-\frac{b^{2}}{a^{2}}\right) w .
\end{array}\right.
$$

We shall show that $z(t ; \varepsilon)$ is relatively small with respect to $v(t ; \varepsilon)$, or more precisely 


$$
\frac{z(t ; \varepsilon)}{v(t ; \varepsilon)}=O\left(\varepsilon^{\frac{1}{2}}\right), \quad \varepsilon+0
$$

uniformly in each interval $0<\delta \leq t \leq T$, where $\delta$ is an arbitrarily small positive constant independent of $\varepsilon$.

Let us assume for the moment that $\mathrm{f}(0)=\ell \neq 0$ (without loss of generality we may assume the constant $\ell$ to be positive). From the differentiability of $f$ it follows that there exists an interval $\left[0, t_{0}\right]$ such that

$$
\frac{1}{2} l \leq f(t) \leq \frac{3}{2} l \quad \text { for } 0 \leq t \leq t_{0} \text {. }
$$

Using this inequality we obtain from (31) the following estimates for $v(t ; \varepsilon)$ which are valid for sufficiently small values of $\varepsilon$ :

$$
\begin{cases}0 \leq v(t ; \varepsilon) \leq A \varepsilon & \text { for } 0 \leq t \leq T \\ v(t ; \varepsilon) \geq B \varepsilon & \text { for } 0<\delta \leq t \leq T .\end{cases}
$$

Here $A$ and $B$ are positive constants independent of $\varepsilon$, and $\delta$ is some arbitrarily small positive constant independent of $\varepsilon$. Furthermore it is easily established that the function F defined by (36), (29) and (33) satisfies

$$
|F(t ; \varepsilon)| \leq C(T) \varepsilon\left\{v(t ; \varepsilon)+|f(t ; \varepsilon)| \exp \left(-\frac{a_{0} t}{\varepsilon}\right)\right.
$$

$$
\left.+\int_{0}^{t}|f(\tau ; \varepsilon)| d \tau \exp \left(-\frac{a_{0} t}{\varepsilon}\right)\right\}
$$

valid for $0 \leq t \leq T$, where $C(T)$ is some generic constant depending on $T$ but not on $\varepsilon$, and $a_{0}=\min [0, T]|a(t)|$.

From this inequality we obtain with the aid of (38) and (39) an estimate for the $\mathrm{L}_{2}$-norm of $\mathrm{F}$, namely

$$
\|F\|_{[0, T]}=0\left(\varepsilon^{3 / 2}\right), \quad \varepsilon+0
$$

so it follows from theorem 1 that the solution $z$ of (35) satisfies

$$
z(t ; \varepsilon)=0\left(\varepsilon^{3 / 2}\right), \quad \varepsilon \downarrow 0
$$

uniformly in $0 \leq t \leq \mathrm{T}$. 
Finally we obtain from (39) and (41)

$$
\frac{z(t ; \varepsilon)}{v(t ; \varepsilon)}=O\left(\varepsilon^{\frac{1}{2}}\right), \quad \varepsilon \downarrow 0
$$

uniformly valid for $0<\delta \leq t \leq T$.

Let us now consider the case where $f(0)=0$.

We assume that there exists an interval $\left(0, t_{0}\right]$ with the property that

$f(t) \neq 0$ for $0<t \leq t_{0}$ (without loss of generality we may assume $f(t)$ to be positive for $0<t \leq t_{0}$ ). In addition it will be assumed that there exist positive constants $C_{1}, C_{2}$ and $\alpha$ such that

$$
c_{1} t^{\alpha} \leq f(t) \leq c_{2} t^{\alpha} \quad \text { for } 0 \leq t \leq t_{0}
$$

(note that necessarily $\alpha \geq 1$ because of the differentiability of $f$ at $t=0$ ). Using (42) we obtain from (31) the following estimates for $v(t ; \varepsilon)$ which are valid for sufficiently small values of $\varepsilon$ :

$$
\begin{cases}0 \leq \mathrm{v}(t ; \varepsilon) \leq A \varepsilon^{\alpha+1} & \text { for } 0 \leq t \leq T \\ \mathrm{v}(t ; \varepsilon) \geq B \varepsilon^{\alpha+1} & \text { for } 0<\delta \leq t \leq \mathrm{T} .\end{cases}
$$

Here $A$ and $B$ are positive constants independent of $\varepsilon$, and $\delta$ is some arbitrarily small positive constant independent of $\varepsilon$. We also obtain from (40), (42) and (43) the estimate

$$
\|F\|_{[0, T]}=0\left(\varepsilon^{\alpha+3 / 2}\right), \quad \varepsilon \downarrow 0
$$

so theorem 1 yields

$$
z(t ; \varepsilon)=0\left(\varepsilon^{\alpha+3 / 2}\right), \quad \varepsilon+0
$$

uniformly valid in $0 \leq t \leq T$.

Finally it follows from (43) and (44) that

$$
\frac{z(t ; \varepsilon)}{v(t ; \varepsilon)}=0\left(\varepsilon^{\frac{1}{2}}\right), \quad \varepsilon \downarrow 0
$$


uniformly valid for $0<\delta \leq t \leq T$.

Using the formulae (28), (31), (34) and (37) we obtain

$$
u(t ; \varepsilon)=w(t)+\left\{1+o\left(\varepsilon^{\frac{1}{2}}\right)\right\} v(t ; \varepsilon) \exp \left[-\frac{1}{\varepsilon} \int_{0}^{t} a(\tau) d \tau\right]
$$

$$
=w(t)-\frac{1+O\left(\varepsilon^{\frac{1}{2}}\right)}{a(t)} \int_{0}^{t} f(\tau) \exp \left\{\int_{\tau}^{t} \frac{b(\xi)}{a(\xi)} d \xi-\frac{1}{\varepsilon} \int_{\tau}^{t} a(\xi) d \xi\right\} d \tau
$$

uniformly valid in every bounded interval $0<\delta \leq t \leq T$.

REMARK. One easily verifies that the solution $w(t)$ of the reduced problem (27) provides a formal approximation for $u(t ; \varepsilon)$ in case $f(0)=0$. However, it follows from formula (45) that $w(t)$ does not approximate $u(t ; \varepsilon)$ for small values of $\varepsilon$ since the difference $u-w$ increases exponentially as $\varepsilon$ tends to zero. Hence we conclude that a formal approximation for the solution $u$ of problem (25) with a negative coefficient $a(t)$ is not necessarily a good approximation.

Finally we obtain from (45)

THEOREM 3. Let $u(t ; \varepsilon)$ be the solution of the initial value problem (25) under the conditions (26) and let the function $f(t)$ satisfy

$$
c_{1} t^{\alpha} \leq f(t) \leq c_{2} t^{\alpha}
$$

in some interval $0 \leq t \leq t_{0}$, where the constants $C_{1}$ and $c_{2}$ are either both positive or both negative and the constant $\alpha$ is nonnegative. Then we have

$$
u(t ; \varepsilon)=-\frac{1+0\left(\varepsilon^{\frac{1}{2}}\right)}{a(t)} \int_{0}^{t} f(\tau) \exp \left\{\int_{\tau}^{t} \frac{b(\xi)}{a(\xi)} d \xi-\frac{1}{\varepsilon} \int_{\tau}^{t} a(\xi) d \xi\right\} d \tau, \quad \varepsilon \downarrow 0
$$

uniformly valid in every bounded interval $0<\delta \leq t \leq T$, where $\delta$ is an arbitrarily small positive constant independent of $\varepsilon$.

COROLLARY. It follows from (39), (43) and (45) that the solution $u(t ; \varepsilon)$ of (25) under the conditions (26) and (46) satisfies

$$
\mathrm{K}_{1} \varepsilon^{\alpha+1} \exp \left\{-\frac{1}{\varepsilon} \int_{0}^{t} a(\tau) d \tau\right\} \leq u(t ; \varepsilon) \leq \mathrm{K}_{2} \varepsilon^{\alpha+1} \exp \left\{-\frac{1}{\varepsilon} \int_{0}^{t} a(\tau) d \tau\right\}
$$


valid for $0<\delta \leq t \leq \mathrm{T}$ and $\varepsilon$ sufficiently small. Here $\mathrm{K}_{1}$ and $\mathrm{K}_{2}$ are $\varepsilon$-independent constants which are either both positive or both negative.

REMARK. Using a result of SMITH [45, p.245] (see also formula (49)) it is possible to replace the term $O\left(\varepsilon^{\frac{1}{2}}\right)$ in the right-hand side of $(47)$ by $O(\varepsilon)$.

\section{Discussion}

The a priori estimates for $u$ and $\frac{d u}{d t}$ that were established in theorem 1 are, under the conditions stated, the sharpest possible in the sense that the $\varepsilon$-dependent factors in front of the quantities $\left\|_{f}\right\|_{[0, T]}\left|C_{1}(\varepsilon)\right|$ and $\left|C_{2}(\varepsilon)\right|$ are the smallest possible. This can easily be checked by considering appropriate initial value problems. As an example we shall prove that the factor $\varepsilon^{-\frac{1}{2}}$ in the right-hand side of $(10 \mathrm{~b})$ is optimal.

Let $\mathrm{u}$ be the solution of the initial value problem

$$
\left\{\begin{array}{l}
\varepsilon \frac{d^{2} u}{d t^{2}}+\frac{d u}{d t}=f(t ; \varepsilon), \quad 0<t<\infty \\
u(0 ; \varepsilon)=u_{t}(0 ; \varepsilon)=0
\end{array}\right.
$$

with

$$
f(t ; \varepsilon)=\exp \left(-\frac{t}{\varepsilon}\right)
$$

It is easily verified that this solution satisfies

$$
\frac{d u}{d t}(t ; \varepsilon)=\frac{t}{\varepsilon} \exp \left(-\frac{t}{\varepsilon}\right)
$$

so it follows from the relations

$$
\left\{\begin{array}{l}
\|f\|_{[0, T]}=0\left(\varepsilon^{\frac{1}{2}}\right), \quad \varepsilon+0 \\
\sup _{[0, T]}\left|\frac{d u}{d t}(t ; \varepsilon)\right|=0(1), \quad \varepsilon+0
\end{array}\right.
$$

that the factor $\varepsilon^{-\frac{1}{2}}$ in the right-hand side of (10b) is the smallest possible. In a similar way the other factors in (10a) and (10b) can be shown to be optimal. 
Alternative methods for obtaining a priori estimates have been given by O'MALLEY [37] and SMITH [45]: an estimate for the solution $u$ of (1) (with a positive coefficient $a(t ; \varepsilon)$ ) is derived by integrating the differential equation twice and then either solving the resulting volterra equation by successive approximations (O'MALLEY) or applying GRONWALL's lemma (SMITH). The latter method yields the estimate

$$
\sup _{[0, T]}|u(t ; \varepsilon)| \leq C(T)\left\{\|f\|_{1,[0, T]}+\left|C_{1}(\varepsilon)\right|+\varepsilon\left|C_{2}(\varepsilon)\right|\right\}
$$

where

$$
\|f\|_{1,[0, T]}=\int_{0}^{T}|f(\tau ; \varepsilon)| d \tau .
$$

This estimate may for certain problems (e.g. problem (48)) be sharper than (10a).

Estimates which are valid in the infinite interval $0 \leq t<\infty$ have been derived by WEINSTEIN \& SMITH [54] for an equation of the type (1) and by HOPPENSTEADT [24] for a nonlinear system of first order equations.

The estimates obtained in section 1 for solutions of linear differential equations will turn out to play an important role in the investigations of nonlinear initial value problems. We shall deal with these problems in chapter II.

The energy integral method underlying the proof of theorem 1 can be generalized to yield a priori estimates for solutions of singularly perturbed initial value problems for hyperbolic differential equations and asymptotic expansions for these solutions can be obtained by a generalization of the expansion technique developed in section 2. This subject will be discussed in chapter III. 
CHAPTER II

INITIAL VALUE PROBLEMS FOR NONLINEAR

ORDINARY DIFFERENTIAL EQUATIONS

In this chapter we shall extend the linear theory developed in chapter I to nonlinear initial value problems. In particular we shall investigate the quasilinear problem

$$
\left\{\begin{array}{l}
\varepsilon \frac{d^{2} u}{d t^{2}}+a(t, u ; \varepsilon) \frac{d u}{d t}+b(t, u ; \varepsilon)=f(t ; \varepsilon), \quad 0<t<\infty \\
u(0 ; \varepsilon)=u_{t}(0 ; \varepsilon)=0
\end{array}\right.
$$

where $\varepsilon$ is a small positive parameter, i.e. $0<\varepsilon<1$.

Under appropriate conditions on the data we shall establish in section 1 the existence of a solution for problem (1), together with an a priori estimate for this solution.

In section 2 we shall construct a formal approximation $\tilde{u}$ for the solution $\mathrm{u}$ of (1). The correctness of this approximation will then be verified with the aid of the results of section 1 .

\section{Existence and a priori estimates}

We shall start this section with the formulation of a theorem on nonlinear operators defined in some normed linear space. This theorem is a modified version of a theorem by VAN HARTEN [23, p.190] and its proof is based on a contraction mapping principle for operators in a Banach space.

Let us define the following spaces:

$\left\{\begin{array}{l}\mathrm{R}: \text { a normed linear space with norm }\|\cdot\| \\ \text { B: a Banach space with norm }|\cdot|\end{array}\right.$ 
Let $\bar{\rho}$ be a certain positive number and let $\Omega(\bar{\rho}) \subset \mathrm{R}$ denote the ball with radius $\bar{\rho}$, that is

$$
\Omega(\bar{\rho})=\{\omega \in R \mid\|\omega\| \leq \bar{\rho}\} .
$$

We consider a nonlinear operator $\mathrm{F}$ from $\Omega(\bar{\rho})$ into B satisfying

$$
F[\varnothing]=\varnothing
$$

where $\varnothing$ denotes the zero element in $\mathrm{R}$ or $\mathrm{B}$.

It is assumed that $\mathrm{F}$ can be split up into a linear part $\mathrm{L}$ and a nonlinear part $\Psi$ :

$$
F[u]=L[u]+\Psi[u] .
$$

The linear operator $L$ can be extended to a map from $R$ into $B$ and this extension will also be denoted by $L$. In addition we assume that the following conditions for the operators $L$ and $\Psi$ are satisfied:

Condition a. $L: R \rightarrow B$ is bijective and the inverse operator $L^{-1}: B \rightarrow R$ is continuous, that is

$$
\left\|L_{L}^{-1}[\mathrm{v}]\right\| \leq \ell^{-1}|\mathrm{v}| \quad \text { for all } \mathrm{v} \in \mathrm{B}
$$

where $l$ is some positive constant.

Condition b. $\Psi: \Omega(\bar{\rho}) \rightarrow$ B satisfies the Lipschitz condition

$$
\left|\Psi\left[\omega_{1}\right]-\Psi\left[\omega_{2}\right]\right| \leq \mathrm{m}(\rho)\left\|\omega_{1}-\omega_{2}\right\|
$$

valid for all $\omega_{1}, \omega_{2} \in \Omega(\rho)$ and all $\rho \in[0, \bar{p}]$.

Here $m(\rho)$ is supposed to be continuous for $\rho \in[0, \bar{\rho}]$ and monotonically decreasing for decreasing $\rho$ with

$$
\lim _{\rho \nmid 0} m(\rho)=0 .
$$

Our problem is to determine an element $u \in \Omega(\bar{\rho})$ which satisfies 


$$
F[u]=f
$$

for a given $f \in B$.

Putting $u=L^{-1}[v]$ we obtain the following equivalence:

$$
F[u]=f, u \in \Omega(\bar{\rho}), f \in B \Leftrightarrow T[v]=v, v \in L[\Omega(\bar{\rho})], \quad f \in B
$$

where the nonlinear operator $\mathrm{T}: \mathrm{L}[\Omega(\bar{\rho})] \rightarrow \mathrm{B}$ is given by

$$
T[v]=f-\Psi \circ L^{-1}[v] .
$$

After these preparations we can formulate

THEOREM 1. Suppose that the nonlinear operator $F: \Omega(\bar{\rho}) \rightarrow B$ with $\Omega(\bar{\rho})$ a closed ball with radius $\bar{\rho}$ in a normed linear space $\mathrm{R}$ (norm $\|\cdot\|$ ) and $\mathrm{B}$ a Banach space (norm $|\cdot|$ ) satisfies the conditions (2) and (3) and the conditions $\mathrm{a}$ and $\mathrm{b}$.

Let $\rho_{0}$ be defined as the largest number $\rho \in[0, \bar{\rho}]$ for which

$$
\mathrm{m}\left(\rho_{0}\right) \leq \frac{1}{2} \ell .
$$

Then problem (4) has for sufficiently small $f \in B$, viz.

$$
|f| \leq \frac{1}{2} \ell_{\rho} \rho^{\prime}
$$

a solution $u$ in the ball $\Omega\left(\rho_{0}\right)$ and this solution satisfies the estimate

$$
\|u\| \leq 2 \ell^{-1}|f|
$$

PROOF. Since problem (4) is equivalent to

$$
T[v]=v, \quad v \in L[\Omega(\bar{\rho})], \quad f \in B
$$

it can be considered as a search for a fixed point of an operator $T$ from $L[\Omega(\bar{\rho})] \subset \mathrm{B}$ into $\mathrm{B}$. The existence of such a fixed point can easily be established. Following VAN HARTEN $\left[23\right.$, p.191] we show that for $2 \ell^{-1}|f| \leq \rho \leq \rho_{0}$ 
(i) $T$ is defined in the ball $B(\ell \rho)=\{v \in B|| v \mid \leq \ell \rho\}$,

(ii) $T$ is a strict contraction on $B\left(\ell_{\rho}\right)$,

(iii) $T\left[B\left(\ell_{\rho}\right)\right] \subset B\left(\ell_{\rho}\right)$.

Ad (i): for any $v \in B\left(l_{p}\right)$ we have

$$
v=L \circ L^{-1}[v] \text { and }\left\|L^{-1}[v]\right\| \leq \ell^{-1}|v| \leq \rho \leq \rho_{0} \leq \bar{\rho}
$$

so it follows that $B\left(\ell_{\rho}\right) \subset L[\Omega(\bar{\rho})]$. Since $T$ is defined in $L[\Omega(\bar{\rho})]$ it is also defined in $B\left(\ell_{\rho}\right)$.

Ad (ii): for $v_{1}, v_{2} \in B\left(l_{p}\right)$ we have

$$
\begin{aligned}
\left|T\left[v_{1}\right]-T\left[v_{2}\right]\right| & \leq\left|\Psi \circ L^{-1}\left[v_{1}\right]-\Psi \circ L^{-1}\left[v_{2}\right]\right| \\
& \leq m(\rho)\left\|L^{-1}\left[v_{1}\right]-L^{-1}\left[v_{2}\right]\right\| \\
& \leq \ell^{-1} m(\rho)\left|v_{1}-v_{2}\right| \\
& \leq \frac{1}{2}\left|v_{1}-v_{2}\right| .
\end{aligned}
$$

Ad (iii): for $v \in B(\ell \rho)$ we have

$$
\begin{aligned}
|\mathrm{T}[\mathrm{v}]| & \leq|f|+\left|\Psi \circ L^{-1}[\mathrm{v}]\right| \\
& \leq \frac{1}{2} l \rho+\mathrm{m}(\rho)\left\|_{L}^{-1}[\mathrm{v}]\right\| \\
& \leq \frac{1}{2} l \rho+\mathrm{m}(\rho) \rho \\
& \leq \ell \rho .
\end{aligned}
$$

It is well known that a strictly contractive map $T$ from a sphere (in a Banach space) into itself has exactly one fixed point in that sphere. This leads to the unique existence of a solution $v \in B(\ell \rho)$. Due to the equivalence there exists also a solution $u \in \Omega(\rho)$ of problem (4); actually we have

$$
\|\mathrm{u}\|=\left\|L_{L}^{-1}[\mathrm{v}]\right\| \leq \ell^{-1}|\mathrm{v}| \leq \rho .
$$


Note that uniqueness of $u$ has not been established in the whole ball $\Omega(\rho)$, but only in $L^{-1}[B(\ell \rho)] \subset \Omega(\rho)$.

Finally, choosing $\rho=\rho_{0}$ we obtain the largest domain for which uniqueness can be guaranteed; choosing $\rho=2 \ell^{-1}|f|$ we find the estimate (5), which concludes the proof of theorem 1 .

The proof given above is, apart from the definition of the operator $T$, identical to the proof of a similar theorem by VAN HARTEN [23, p.191]. The difference between VAN HARTEN's theorem and ours is formed by the definition of the operator $\mathrm{F}$ : in our case $\mathrm{F}$ maps a ball belonging to a normed linear space into a Banach space, whereas VAN HARTEN considers an operator $F$ which maps a (linear subspace of a) Banach space into a normed linear space.

Our formulation has the advantage that the theory is applicable to more general operators $F$, since $F$ is allowed to be defined only in a ball instead of in a linear subspace. This fact will enable us for instance to consider quasilinear differential operators $F$ with coefficients having singularities when regarded as functions of $u$.

Another advantage is lying in the fact that $F$ is defined in a subset of a normed linear space instead of in a subset of a Banach space. This will become clear when we apply theorem 1 to kyperbolic differential equations containing a quasilinear first order part (see chapter IV, section 1).

After these preliminaries we turn our attention to the initial value problem (1). We impose the following conditions on the data:

there exists a positive constant $T_{1}$ such that for each positive constant $T$ satisfying $T<T_{1}$ there exists a positive constant $\varepsilon_{1}$ (depending on $T$ ) such that

(6)

(i) $\quad a(t, 0 ; \varepsilon) \in c^{1}[0, T], \quad b_{u}(t, 0 ; \varepsilon) \in C^{0}[0, T], \quad f(t ; \varepsilon) \in C^{0}[0, T]$ valid for every value of $\varepsilon$ satisfying $0<\varepsilon \leq \varepsilon_{1}$

(ii) the function $a(t, 0 ; \varepsilon)$ and its first derivative $\frac{\partial a}{\partial t}(t, 0 ; \varepsilon)$ as well as the function $b_{u}(t, 0 ; \varepsilon)$ are uniformly bounded in $0 \leq t \leq T$ for $0<\varepsilon \leq \varepsilon_{1}$ 

(6) $\mid \begin{array}{ll}\text { (iii) } a(t, 0 ; \varepsilon) \geq a_{0}>0 \\ \text { valid for all } t \in[0, T] \text { and all } \varepsilon \epsilon\left(0, \varepsilon_{1}\right], \text { where } a_{0} \text { is some } \\ \text { constant depending on } T \text { and } \varepsilon_{1} \text { but independent of } \varepsilon\end{array}$

there exists a positive constant $\mathrm{T}_{2}$ such that for each positive constant $T$ satisfying $T<T_{2}$ there exist a positive constant $U_{0}$ and $a$ positive constant $\varepsilon_{2}$ (depending on $T$ and $U_{0}$ ) such that

(i) $\quad a(t, u ; \varepsilon) \in c^{0}[0, T], \quad b(t, u ; \varepsilon) \in c^{0}[0, T]$

valid for all $u \in\left[-\mathrm{U}_{0}, \mathrm{U}_{0}\right]$ and all $\varepsilon \in\left(0, \varepsilon_{2}\right]$

(7)

(ii) the derivative $a_{u}(t, u ; \varepsilon)$ exists and is uniformly bounded in $[0, T] \times\left[-U_{0}, U_{0}\right]$ for $0<\varepsilon \leq \varepsilon_{2}$

(iii) the derivative $b_{u}(t, u ; \varepsilon)$ exists in $[0, T] \times\left[-U_{0}, U_{0}\right]$ for $0<\varepsilon \leq \varepsilon_{2}$ and satisfies a Lipschitz condition with exponent $\alpha>0$, viz.

$$
\left|b_{u}(t, u ; \varepsilon)-b_{u}(t, 0 ; \varepsilon)\right| \leq C\left(T, U_{0}, \varepsilon_{2}\right)|u|^{\alpha}
$$

valid for all $(t, u) \in[0, T] \times\left[-U_{0}, U_{0}\right]$ and all $\varepsilon \in\left(0, \varepsilon_{2}\right]$. Here $C\left(T, U_{0}, \varepsilon_{2}\right)$ is some positive constant depending on $T, U_{0}$ and $\varepsilon_{2}$ but independent of $t$, $u$ or $\varepsilon$.

It follows from the conditions $(6, i)$ and $(7, i)$ that we may assume without loss of generality that

$$
b(t, 0 ; \varepsilon) \equiv 0 \text { for } 0 \leq t<\infty \text { and } 0<\varepsilon<<1 \text {. }
$$

The existence of a solution for problem (1) can now be established by applying theorem 1. In terms of this theorem we introduce for any positive $T$ with $T<T_{0}=\min \left(T_{1}, T_{2}\right)$ and for any fixed value of $\varepsilon$ with $0<\varepsilon \leq \varepsilon_{0}=\min \left(\varepsilon_{1}, \varepsilon_{2}\right)$ the Banach space

$$
B=\left\{u \mid u \in C^{0}[0, T]\right\}
$$




$$
|u|_{0}=\sup _{[0, T]}|u|
$$

and the normed linear space

$$
R=\left\{u \mid u \in C^{2}[0, T], u(0)=u_{t}(0)=0\right\}
$$

with norm

$$
|u|_{1}^{(\varepsilon)}=\sup _{[0, T]}|u|+\varepsilon^{\frac{1}{2}} \sup _{[0, T]}\left|u_{t}\right|
$$

Choosing $\bar{\rho}=\mathrm{U}_{0}$ we define the operator $\mathrm{F}: \Omega(\bar{\rho}) \rightarrow \mathrm{B}$ by

$$
F[u]=\varepsilon \frac{d^{2} u}{d t^{2}}+a(t, u ; \varepsilon) \frac{d u}{d t}+b(t, u ; \varepsilon)
$$

which according to (8) satisfies the relation (2). For the operator $L: R \rightarrow B$ we take the linearization of $\mathrm{F}$ in the origin, thus

$$
L[u]=\varepsilon \frac{d^{2} u}{d t^{2}}+a(t, 0 ; \varepsilon) \frac{d u}{d t}+b_{u}(t, 0 ; \varepsilon) u \text {. }
$$

Then the operator $\Psi: \Omega(\bar{\rho}) \rightarrow$ B is given, by

$$
\begin{aligned}
\Psi[u]=F[u]-L[u]= & \{a(t, u ; \varepsilon)-a(t, 0 ; \varepsilon)\} \frac{d u}{d t} \\
& +b(t, u ; \varepsilon)-b_{u}(t, 0 ; \varepsilon) u
\end{aligned}
$$

Using the conditions (6) we obtain from theorem 1 in chapter I (and from the first remark following that theorem)

$$
\left|L^{-1}[v]\right|_{1}^{(\varepsilon)} \leq M(T)|v|_{0}
$$

valid for all $\varepsilon \in\left(0, \varepsilon_{0}\right]$ and all $v \in B$, where $M(T)$ is some positive constant depending on $T$ but independent of $\varepsilon$, and $\varepsilon_{0}$ is some generic constant. So in terms of theorem 1 we have

$$
\ell=\frac{1}{M(T)}
$$

It can easily be verified that the operator $\Psi$ satisfies a Lipschitz condition. Using the conditions (7) we obtain for every $\rho \in[0, \bar{\rho}]$ and every 
$\mathrm{v}, \mathrm{w} \in \mathrm{R}$ with $|\mathrm{v}|_{1}^{(\varepsilon)} \leq \rho,|\mathrm{w}|_{1}^{(\varepsilon)} \leq \rho$ the following relation

$$
\begin{aligned}
\Psi[v]-\Psi[w]= & \{a(t, v ; \varepsilon)-a(t, 0 ; \varepsilon)\}\left(v_{t}-w_{t}\right) \\
& +w_{t}\{a(t, v ; \varepsilon)-a(t, w ; \varepsilon)\} \\
& +b(t, v ; \varepsilon)-b(t, w ; \varepsilon)-b_{u}(t, 0 ; \varepsilon)(v-w) \\
= & v a_{u}(t, \theta, v ; \varepsilon)\left(v_{t}-w_{t}\right)+w_{t} a_{u}\left(t, w+\theta_{2}(v-w) ; \varepsilon\right)(v-w) \\
& +\left\{b_{u}\left(t, w+\theta_{3}(v-w) ; \varepsilon\right)-b_{u}(t, 0 ; \varepsilon)\right\}(v-w)
\end{aligned}
$$

with $0<\theta_{i}<1(i=1,2,3)$. Hence it follows that

$$
\begin{aligned}
|\Psi[\mathrm{v}]-\Psi[\mathrm{w}]|_{0} & \leq \mathrm{C}(\mathrm{T})\left\{\rho \sup _{[0, \mathrm{~T}]}\left|\mathrm{v}_{t}-\mathrm{w}_{t}\right|+\max \left(\varepsilon^{-\frac{1}{2}} \rho, \rho^{\alpha}\right) \sup _{[0, \mathrm{~T}]}|\mathrm{v}-\mathrm{w}|\right\} \\
& \leq C(T) \max \left(\varepsilon^{-\frac{1}{2} \rho, \rho^{\alpha}}\right)|\mathrm{v}-\mathrm{w}|_{1}^{(\varepsilon)}
\end{aligned}
$$

valid for $0<\varepsilon \leq \varepsilon_{0}$. Here $C(T)$ is a positive constant which depends (apart from $\mathrm{U}_{0}$ and $\varepsilon_{0}$ ) on $\mathrm{T}$ but not on $\varepsilon$.

So still using the terminology of theorem 1 we conclude that

$$
m(\rho)=C(T) \max \left(\varepsilon^{-\frac{1}{2}} \rho, \rho^{\alpha}\right)
$$

N.B. The following simplifications are easily established:

if a does not depend on $u$ then $m(\rho)=C(T) \rho^{\alpha}$,

if $b$ is linear in $u$ then $m(\rho)=C(T) \varepsilon^{-\frac{1}{2}} \rho$.

Solving $m\left(\rho_{0}\right)=\frac{1}{2} l$ we obtain

$$
\rho_{0}=N(T) \varepsilon^{\lambda}
$$

with

$$
\lambda= \begin{cases}0 & \text { if a does not depend on } u \\ \frac{1}{2} & \text { otherwise }\end{cases}
$$


and $\mathrm{N}(\mathrm{T})$ some positive constant depending on $\mathrm{T}$ but independent of $\varepsilon$.

Finally, applying theorem 1 we obtain

THEOREM 2. Let the functions $\mathrm{a}(t, \mathrm{u} ; \varepsilon), \mathrm{b}(t, \mathrm{u} ; \varepsilon)$ and $\mathrm{f}(t ; \varepsilon)$ satisfy the conditions (6), (7) and (8), and let $\mathrm{T}$ be some positive constant satisfying $\mathrm{T}<\mathrm{T}_{0}=\min \left(\mathrm{T}_{1}, \mathrm{~T}_{2}\right)$. Then there exist positive constants $\varepsilon_{0}, \mathrm{M}(\mathrm{T})$ and $\mathrm{N}(\mathrm{T})$ (depending on $\mathrm{T}$ but independent of $\varepsilon$ ) such that for $0 \leq t \leq \mathrm{T}<\mathrm{T}_{0}$ and $0<\varepsilon \leq \varepsilon_{0}$ problem (1) has a solution $u \in R$, provided that $f$ is sufficiently small, viz.

$$
\sup _{[0, T]}|f| \leq \frac{N(T)}{2 M(T)} \varepsilon^{\lambda} .
$$

Here the normed linear space $\mathrm{R}$ is given by (9) and (10), and the nonnegative constant $\lambda$ is given by (11).

The solution $\mathrm{u}$ satisfies the estimate

$$
|u|_{1}^{(\varepsilon)} \leq 2 M(T) \sup _{[0, T]}|f|
$$

REMARKS.

1. Note that the coefficients $a(t, u ; \varepsilon)$ and $b(t, u ; \varepsilon)$ are allowed to have singularities in $u$. For instance, if

$$
a(t, u ; \varepsilon)=\frac{1}{1-u}
$$

we choose the positive constant $U_{0}$ mentioned in ( 7 ) such that $U_{0}<1$. With this choice the conditions (6) and (7) for $a(t, u ; \varepsilon)$ are fulfilled, as one easily verifies.

2. A similar theorem can be formulated for more general nonlinear initial value problems of the type

$$
\left\{\begin{array}{l}
\varepsilon \frac{d^{2} u}{d t^{2}}+F(t, \vec{u} ; \varepsilon)=f(t ; \varepsilon), \quad 0<t<\infty \\
u(0 ; \varepsilon)=u_{t}(0 ; \varepsilon)=0
\end{array}\right.
$$

(where $\vec{u}$ denotes the vector $\left(u, u_{t}\right)$ ) provided that suitable conditions are imposed on the nonlinear function $F$, namely 
(i)

$$
F(t, \overrightarrow{0} ; \varepsilon) \equiv 0 \text { for } 0 \leq t<\infty \text { and } 0<\varepsilon<1 \text {, }
$$

(ii) in order that theorem 1 of chapter I is applicable to the linearized problem one must require that $(6)$ holds, with $a(t, 0 ; \varepsilon)$ and $b_{u}(t, 0 ; \varepsilon)$ replaced by $F_{u_{t}}(t, \overrightarrow{0} ; \varepsilon)$ respectively $F_{u}(t, \overrightarrow{0} ; \varepsilon)$,

(iii) in order that the nonlinear part $\Psi$ given by

$$
\Psi[u]=F(t, \vec{u} ; \varepsilon)-F_{u_{t}}(t, \overrightarrow{0} ; \varepsilon) \frac{d u}{d t}-F_{u}(t, \overrightarrow{0} ; \varepsilon) u
$$

satisfies a Lipschitz condition it is sufficient to require that there exist positive constants $T, U_{0}$ and $\varepsilon_{0}$ such that the functions $F, F_{u u^{\prime}} F_{u_{u}}$ and $F_{u_{t} u_{t}}$ are continuous and uniformly bounded in $0 \leq t \leq T,|u| \leq U_{0},\left|u_{t}\right| \leq U_{0}$ for $0<\varepsilon \leq \varepsilon_{0}$.

\section{Asymptotic expansions}

As an application of the a priori estimates obtained in the foregoing section we shall construct an asymptotic expansion into powers of $\varepsilon$ for the solution $u$ of the quasilinear initial value problem

$$
\left\{\begin{array}{l}
L^{(\varepsilon)}[u] \equiv \varepsilon \frac{d^{2} u}{d t^{2}}+a(t, u) \frac{d u}{d t}+b(t, u)=0, \quad 0<t<\infty \\
u(0 ; \varepsilon)=C_{1}, \quad u_{t}(0 ; \varepsilon)=C_{2} .
\end{array}\right.
$$

For simplicity it will be assumed that all data are $C^{\infty}$-functions, that is

$$
\left.a(t, u) \in C^{\infty} \overline{\left(\mathbb{R}^{+}\right.} \times \mathbb{R}\right), \quad b(t, u) \in C^{\infty}\left(\overline{\mathbb{R}^{+}} \times \mathbb{R}\right)
$$

In addition we assume that

$$
a(t, u)>0 \quad \text { for } 0 \leq t<\infty \text { and }-\infty<u<\infty \text {. }
$$

However, we emphasize that the conditions (13) and (14) are not necessary and can be weakened considerably (see the remarks following theorem 3 of this section).

According to a standard procedure in singular perturbation theory a formal approximation $\tilde{u}$ for the solution $u$ of problem (12) can be constructed by 
putting

$$
\tilde{u}(t ; \varepsilon)=w_{0}(t)+\varepsilon v_{0}(\tau)+\varepsilon w_{1}(t)+\varepsilon^{2} v_{1}(\tau)
$$

where $\tau=\frac{t}{\varepsilon}$ and $w_{k}$ and $v_{k}(k=0,1)$ are functions which have to be determined. For the moment we shall assume that these functions have the following properties

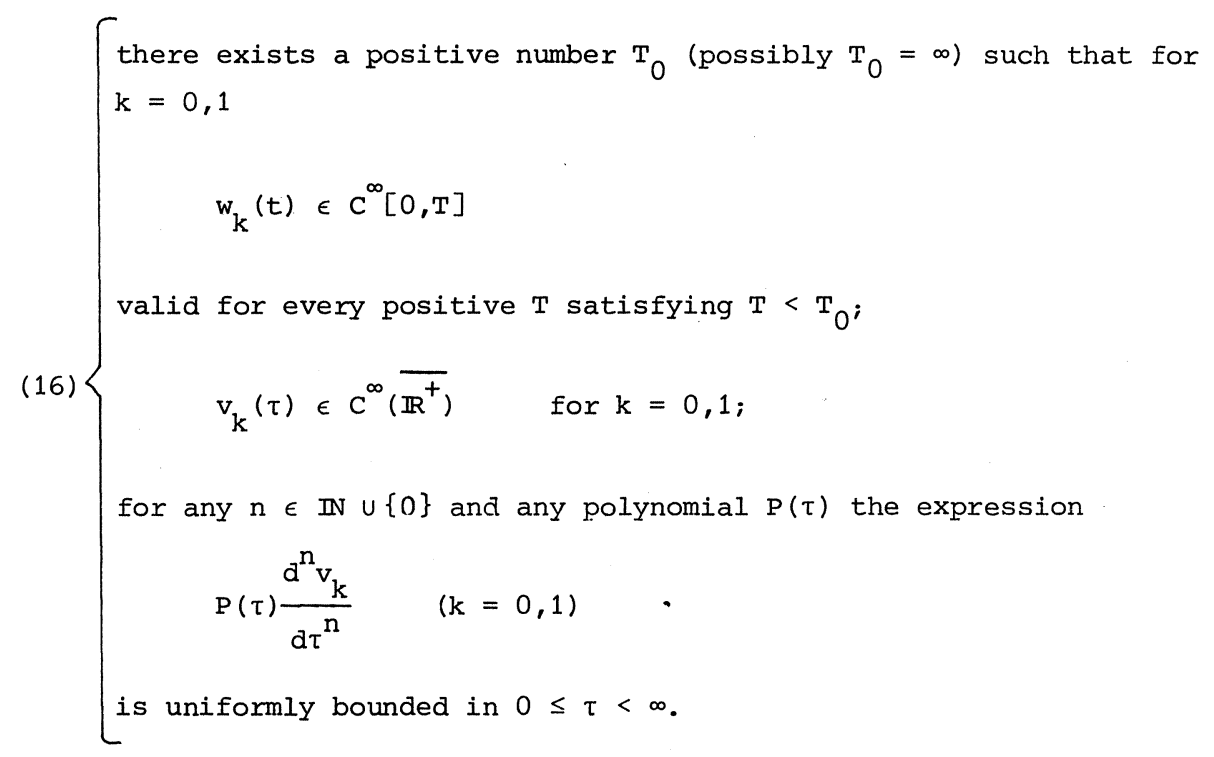

We shall verify the validity of these properties later on, when the functions $\mathrm{w}_{\mathrm{k}}$ and $\mathrm{v}_{\mathrm{k}}$ have been determined explicitly.

Let $\mathrm{T}$ be some positive number satisfying $\mathrm{T}<\mathrm{T}_{0}$. Expanding the coefficient $a(t, \tilde{u})$ we obtain using (15) and (16)

$$
\begin{aligned}
a(t, \tilde{u}) & =a\left(t, w_{0}+\varepsilon v_{0}+\varepsilon w_{1}+\varepsilon^{2} v_{1}\right) \\
& =a\left(t, w_{0}\right)+\varepsilon\left(v_{0}+w_{1}\right) \frac{\partial a}{\partial u}\left(t, w_{0}\right)+o\left(\varepsilon^{2}\right)
\end{aligned}
$$

uniformly valid in $0 \leq t \leq \mathrm{T}<\mathrm{T}_{0}$.

Using

$$
\frac{d \tilde{u}}{d t}=\frac{d w_{0}}{d t}+\frac{d v_{0}}{d \tau}+\varepsilon \frac{d w_{1}}{d t}+\varepsilon \frac{d v_{1}}{d \tau}
$$


we get

$$
\begin{aligned}
a(t, \tilde{u}) \frac{d \tilde{u}}{d t}= & a\left(t, w_{0}\right) \frac{d w_{0}}{d t}+a\left(t, w_{0}\right) \frac{d v_{0}}{d \tau} \\
& +\varepsilon\left\{a\left(t, w_{0}\right) \frac{d w_{1}}{d t}+a\left(t, w_{0}\right) \frac{d v_{1}}{d \tau}\right\} \\
& +\varepsilon\left(w_{1}+v_{0}\right)\left(\frac{d w_{0}}{d t}+\frac{d v_{0}}{d \tau}\right) \frac{\partial a}{\partial u}\left(t, w_{0}\right) \\
& +o\left(\varepsilon^{2}\right)
\end{aligned}
$$

uniformly valid in $0 \leq t \leq T<T_{0}$.

So putting $t=\varepsilon \tau$ and expanding into powers of $\varepsilon$ we arrive at

$$
\begin{aligned}
a(t, \tilde{u}) \frac{d \tilde{u}}{d t}=a\left(t, w_{0}\right) \frac{d w_{0}}{d t}+a\left(0, w_{0}(0)\right) \frac{d v_{0}}{d \tau} \\
+\varepsilon\left[\left\{\frac{\partial a}{\partial t}\left(0, w_{0}(0)\right)+\frac{\partial a}{\partial u}\left(0, w_{0}(0)\right) \frac{d w_{0}}{d t}(0)\right\} \tau \frac{d v_{0}}{d \tau}\right. \\
+a\left(t, w_{0}\right) \frac{d w_{1}}{d t}+a\left(0, w_{0}(0)\right) \frac{d v_{1}}{d \tau} \\
+\frac{\partial a}{\partial u}\left(t, w_{0}\right) w_{1} \frac{d w_{0}}{d t}+\frac{\partial a}{\partial u}\left(0, w_{0}(0)\right) w_{1}(0) \frac{d v_{0}}{d \tau} \\
\left.+\frac{\partial a}{\partial u}\left(0, w_{0}(0)\right) \frac{d w_{0}}{d t}(0) v_{0}+\frac{\partial a}{\partial u}\left(0, w_{0}(0)\right) v_{0} \frac{d v_{0}}{d \tau}\right] \\
+0\left(\varepsilon^{2}\right)
\end{aligned}
$$

uniformly valid in $0 \leq t \leq T<T_{0}$.

N.B. Unless otherwise stated the argument of $w_{0}, \frac{d w_{0}}{d t}, w_{1}, \frac{d w_{1}}{d t}$ is $t$ and the argument of $v_{0}, \frac{d v_{0}}{d \tau}, v_{1}, \frac{d v_{1}}{d \tau}$ is $\tau$.

A similar expansion of the other terms in $L^{(\varepsilon)}[\tilde{u}]$ leads to the following expansion of the operator: 


$$
\begin{aligned}
L^{(\varepsilon)}[\tilde{u}]= & a\left(t, w_{0}\right) \frac{d w_{0}}{d t}+b\left(t, w_{0}\right)+\frac{d^{2} v_{0}}{d \tau^{2}}+a\left(0, w_{0}(0)\right) \frac{d v_{0}}{d \tau} \\
& +\varepsilon\left[a\left(t, w_{0}\right) \frac{d w_{1}}{d t}+\tilde{b}\left(t, w_{0}\right) w_{1}+\Phi_{1}(t)\right] \\
& +\varepsilon\left[\frac{d^{2} v_{1}}{d \tau^{2}}+a\left(0, w_{0}(0)\right) \frac{d v_{1}}{d \tau}+\Psi_{1}(\tau)\right]+0\left(\varepsilon^{2}\right)
\end{aligned}
$$

uniformly valid in $0 \leq t \leq T<T_{0}$, where

$$
\left\{\begin{aligned}
\tilde{b}\left(t, w_{0}\right) & =\frac{\partial a}{\partial u}\left(t, w_{0}\right) \frac{d w_{0}}{d t}+\frac{\partial b}{\partial u}\left(t, w_{0}\right) \\
\Phi_{1}(t)= & \frac{d^{2} w_{0}}{d t^{2}} \\
\Psi_{1}(\tau)= & \left\{\frac{\partial a}{\partial u}\left(0, w_{0}(0)\right) \frac{d w_{0}}{d t}(0)+\frac{\partial b}{\partial u}\left(0, w_{0}(0)\right)\right\} v_{0} \\
& +\left[\left\{\frac{\partial a}{\partial t}\left(0, w_{0}(0)\right)+\frac{\partial a}{\partial u}\left(0, w_{0}(0)\right) \frac{d w_{0}}{d t}(0)\right\} \tau\right. \\
& \left.+\frac{\partial a}{\partial u}\left(0, w_{0}(0)\right) w_{1}(0)\right] \frac{d v_{0}}{d \tau} \\
& +\frac{\partial a}{\partial u}\left(0, w_{0}(0)\right) v_{0} \frac{d v_{0}}{d \tau} \cdot
\end{aligned}\right.
$$

Now we take for $w_{0}$ the solution of the nonlinear initial value problem

$$
\left\{\begin{array}{l}
a\left(t, w_{0}\right) \frac{d w_{0}}{d t}+b\left(t, w_{0}\right)=0, \quad t>0 \\
w_{0}(0)=c_{1} .
\end{array}\right.
$$

Note that this solution does not necessarily belong to the class $C^{\infty}\left(\overline{\mathbb{R}^{+}}\right)$but may have singularities for finite values of $t$. As an example consider the case where

$$
a\left(t, w_{0}\right) \equiv 1, \quad b\left(t, w_{0}\right)=-w_{0}^{2}, c_{1}=1
$$

The solution is given by $w_{0}=\frac{1}{1-t}$ and has a singularity at $t=1$.

We shall denote by $T_{0}$ the smallest value of $t$ for which the solution $w_{0}$ of (19) becomes singular $\left(\mathrm{T}_{0}=\infty\right.$ if $\mathrm{w}_{0}$ has no singularities). Then we have 
$\mathrm{w}_{0} \in \mathrm{C}^{\infty}[0, \mathrm{~T}]$ for each positive $\mathrm{T}$ with $\mathrm{T}<\mathrm{T}_{0}$.

The functions $v_{0}, w_{1}$ and $v_{1}$ can now be obtained by solving the following set of initial value problems

(20) $\left\{\begin{array}{l}\frac{d^{2} v_{0}}{d \tau^{2}}+a\left(0, w_{0}(0)\right) \frac{d v_{0}}{d \tau}=0, \quad 0<\tau<\infty \\ \frac{d v_{0}}{d \tau}(0)=c_{2}-\frac{d w_{0}}{d t}(0) \\ \lim _{\tau \rightarrow \infty} v_{0}(\tau)=0\end{array}\right.$

(21)

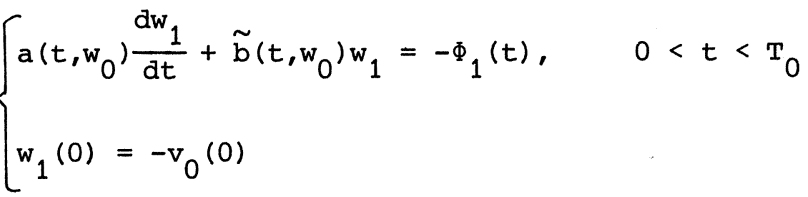

$$
\left\{\begin{array}{l}
\frac{d^{2} v_{1}}{d \tau^{2}}+a\left(0, w_{0}(0)\right) \frac{d v_{1}}{d \tau}=-\Psi_{1}(\tau), \quad 0<\tau<\infty \\
\frac{d v_{1}}{d \tau}(0)=-\frac{d w_{1}}{d t}(0) \\
\lim _{\tau \rightarrow \infty} v_{1}(\tau)=0 .
\end{array}\right.
$$

Note that only the first term $w_{0}$ of the expansion of $\tilde{u}$ follows from the solution of a nonlinear initial value problem; the remaining functions $v_{0}, w_{1}$ and $v_{1}$ satisfy linear equations which can easily be solved.

It is not difficult to verify that the conditions (16) are satisfied. In particular, the functions $v_{0}$ and $v_{1}$ have the form

$$
\left\{\begin{array}{l}
v_{0}(\tau)=\frac{\frac{d w_{0}}{d t}(0)-c_{2}}{a\left(0, w_{0}(0)\right)} \exp \left[-a\left(0, w_{0}(0)\right) \tau\right] \\
v_{1}(\tau)=P_{1}(\tau) \exp \left[-a\left(0, w_{0}(0)\right) \tau\right]+P_{2}(\tau) \exp \left[-2 a\left(0, w_{0}(0)\right) \tau\right]
\end{array}\right.
$$


with $\mathrm{P}_{\mathrm{k}}(\tau) \quad(\mathrm{k}=1,2)$ polynomials in $\tau$. So we see that $\mathrm{v}_{0}$ and $\mathrm{v}_{1}$ are typical boundary layer functions.

Finally, we obtain from (15), (17), (19), (20), (21) and (22) the result

$$
\left\{\begin{array}{l}
L^{(\varepsilon)}[\tilde{u}]=R(t ; \varepsilon), \quad 0<t<T<T_{0} \\
\tilde{u}(0 ; \varepsilon)=C_{1}+\varepsilon^{2} v_{1}(0) \\
\tilde{u}_{t}(0 ; \varepsilon)=C_{2}
\end{array}\right.
$$

where $R(t ; \varepsilon)$ is some function satisfying

$$
R(t ; \varepsilon)=O\left(\varepsilon^{2}\right), \quad \varepsilon+0
$$

uniformly in $0 \leq t \leq T<T_{0}$.

Hence the function $\tilde{u}(t ; \varepsilon)$ defined by $(15),(18),(19),(20),(21)$ and (22) provides a formal approximation (up to order $\varepsilon^{2}$ ) for the solution $u$ of problem (12).

Higher order approximations can be obtained by considering

$$
\tilde{u}(t ; \varepsilon)=\sum_{k=0}^{n} \varepsilon^{k} w_{k}(t)+\sum_{k=0}^{n} \varepsilon^{k+1} v_{k}(\tau), \quad n \in \mathbb{N}, \quad n \geq 2 .
$$

In that case it can be shown that $w_{k}$ and $v_{k}(k=0,1)$ are given by (18), (19), (20), (21) and (22), whereas $w_{k}$ and $v_{k}(k \geq 2)$ follow from the linear problems

$$
\left\{\begin{array}{l}
a\left(t, w_{0}\right) \frac{d w_{k}}{d t}+\tilde{b}\left(t, w_{0}\right) w_{k}=-\Phi_{k}(t), \quad 0<t<T_{0} \\
w_{k}(0)=-v_{k-1}(0)
\end{array}\right.
$$

respectively

$$
\left\{\begin{array}{l}
\frac{d^{2} v_{k}}{d \tau^{2}}+a\left(0, w_{0}(0)\right) \frac{d v_{k}}{d \tau}=-\Psi_{k}(\tau), \quad 0<\tau<\infty \\
\frac{d v_{k}}{d \tau}(0)=-\frac{d w_{k}}{d t}(0) \\
\lim _{\tau \rightarrow \infty} v_{k}(\tau)=0,
\end{array}\right.
$$


the functions $\Phi_{k}$ and $\Psi_{k}(k \in \mathbb{N}, k \geq 2)$ being completely determined by the functions $\mathrm{w}_{0}, \mathrm{w}_{1}, \ldots, \mathrm{w}_{\mathrm{k}-1}$ respectively the functions $\mathrm{w}_{0}, \mathrm{w}_{1}, \ldots, \mathrm{w}_{\mathrm{k}}$ and $\mathrm{v}_{0}, \mathrm{v}_{1}, \ldots, \mathrm{v}_{\mathrm{k}-1}$.

The functions $v_{k}(\tau)(k \in \mathbb{N} \cup\{0\})$ are boundary layer functions of the type

$$
v_{k}(\tau)=\sum_{j=1}^{k+1} P_{j}^{(k)}(\tau) \exp \left[-j a\left(0, w_{0}(0)\right) \tau\right]
$$

with $\mathrm{P}_{j}^{(\mathrm{k})}(\tau)$ a polynomial in $\tau$.

It follows that

$$
\left\{\begin{array}{l}
L^{(\varepsilon)}[\tilde{u}]=R(t ; \varepsilon), \quad 0<t<T<T_{0} \\
\tilde{u}(0 ; \varepsilon)=C_{1}+\varepsilon^{n+1} v_{n}(0) \\
\tilde{u}_{t}(0 ; \varepsilon)=C_{2}
\end{array}\right.
$$

with

$$
R(t ; \varepsilon)=0\left(\varepsilon^{n+1}\right), \quad \varepsilon \downarrow 0
$$

uniformly valid in $0 \leq t \leq T<T_{0}$.

Next we shall prove that a formal approximation $\tilde{u}$ is indeed a good approximation for the solution $u$ of (12). To this end we put

$$
z(t ; \varepsilon)=u(t ; \varepsilon)-\tilde{u}(t ; \varepsilon)
$$

Then we obtain from (12) and (27)

$$
\left\{\begin{array}{l}
\varepsilon \frac{d^{2} z}{d t^{2}}+a(t, z+\tilde{u}) \frac{d z}{d t}+F(t, z ; \varepsilon)=-R(t ; \varepsilon), 0<t<T<T_{0} \\
z(0 ; \varepsilon)=-\varepsilon^{n+1} v_{n}(0) \\
z_{t}(0 ; \varepsilon)=0
\end{array}\right.
$$

where

$$
F(t, z ; \varepsilon)=\{a(t, z+\tilde{u})-a(t, \tilde{u})\} \frac{d \tilde{u}}{d t}+b(t, z+\tilde{u})-b(t, \tilde{u}) .
$$


Defining a function $\tilde{z}(t ; \varepsilon)$ by

$$
\tilde{z}(t ; \varepsilon)=z(t ; \varepsilon)+\varepsilon^{n+1} v_{n}(0)
$$

we obtain

$$
\left\{\begin{array}{l}
\varepsilon \frac{d^{2} \tilde{z}}{d t^{2}}+a^{*}(t, \tilde{z} ; \varepsilon) \frac{d \tilde{z}}{d t}+b^{*}(t, \tilde{z} ; \varepsilon)=-R^{*}(t ; \varepsilon), \quad 0<t<T<T_{0} \\
\tilde{z}(0 ; \varepsilon)=\tilde{z}_{t}(0 ; \varepsilon)=0
\end{array}\right.
$$

where

$$
\left\{\begin{aligned}
a^{*}(t, \tilde{z} ; \varepsilon)= & a\left(t, \tilde{z}+\tilde{u}-\varepsilon^{n+1} v_{n}(0)\right) \\
b^{*}(t, \tilde{z} ; \varepsilon)= & \left\{a\left(t, \tilde{z}+\tilde{u}-\varepsilon^{n+1} v_{n}(0)\right)-a\left(t, \tilde{u}-\varepsilon v^{n+1} v_{n}(0)\right)\right\} \frac{d \tilde{u}}{d t} \\
& +b\left(t, \tilde{z}+\tilde{u}-\varepsilon^{n+1} v_{n}(0)\right)-b\left(t, \tilde{u}-\varepsilon^{n+1} v_{n}(0)\right) \\
R^{*}(t ; \varepsilon)= & R(t ; \varepsilon)+\left\{a\left(t, \tilde{u}-\varepsilon^{n+1} v_{n}(0)\right)-a(t, \tilde{u})\right\} \frac{d \tilde{u}}{d t} \\
& +b\left(t, \tilde{u}-\varepsilon{ }^{n+1} v_{n}(0)\right)-b(t, \tilde{u}) .
\end{aligned}\right.
$$

It is easily established that the functions $a^{*}, b^{*}$ and $R^{*}$ satisfy the conditions (6), (7) and (8) of theorem 2 (with Lipschitz exponent $\alpha=1$ ) and that

$$
R^{*}(t ; \varepsilon)=0\left(\varepsilon^{n+1}\right), \quad \varepsilon+0
$$

uniformly valid in $0 \leq t \leq T<\mathrm{T}_{0}$.

So according to theorem 2 there exists for sufficiently small values of $\varepsilon$ a solution $\tilde{z}$ of (30) which satisfies

$$
|\tilde{z}|_{1}^{(\varepsilon)} \leq C(T) \varepsilon^{n+1}
$$

Here $C(T)$ is some positive constant depending on $T$ but independent of $\varepsilon$. Using (23), (28) and (29) we obtain from the estimate (31) the following theorem, which is a nonlinear generalization of theorem 2 of chapter I: 
THEOREM 3. Let the functions $\mathrm{a}(t, \mathrm{u})$ and $\mathrm{b}(t, \mathrm{u})$ satisfy the conditions (13) and (14), and let $\mathrm{w}_{0}$ be the solution of the nonlinear initial value problem (19). Let $\mathrm{T}_{0}$ denote the smallest value of $t$ for which $\mathrm{w}_{0}$ becomes singular $\left(\mathrm{T}_{0}=\infty\right.$ if $\mathrm{w}_{0}$ has no singularities).

Then there exists for $0 \leq \mathrm{t} \leq \mathrm{T}<\mathrm{T}_{0}$ and for sufficiently small values of $\varepsilon$ a solution $\mathrm{u}$ of problem (12). This solution and its derivative satisfy for any $\mathrm{n} \in \mathbb{I N} \cup\{0\}$ the relations

$$
\begin{cases}u(t ; \varepsilon)=\sum_{k=0}^{n} \varepsilon^{k} w_{k}(t)+\sum_{k=0}^{n-1} \varepsilon^{k+1} v_{k}(\tau)+0\left(\varepsilon^{n+1}\right), & \varepsilon \downarrow 0 \\ \frac{d u}{d t}(t ; \varepsilon)=\sum_{k=0}^{n} \varepsilon^{k} \frac{d w_{k}}{d t}(t)+\sum_{k=0}^{n} \varepsilon^{k} \frac{d v_{k}}{d \tau}(\tau)+0\left(\varepsilon^{n+1}\right), & \varepsilon+0\end{cases}
$$

uniformly in every bounded interval $0 \leq \mathrm{t} \leq \mathrm{T}<\mathrm{T}_{0}$; the functions $\mathrm{w}_{\mathrm{k}}$ and $\mathrm{v}_{\mathrm{k}}$ $(\mathrm{k}=0,1, \ldots, \mathrm{n})$ follow successively from $(19),(20),(21),(22),(24)$ and (25) where $\tau$ is defined by $\tau=\frac{t}{\varepsilon}$.

In case $\mathrm{n}=0$ empty sums are to be interpreted as zero.

COROLLARY 1. It follows from (32) that first approximations ( $\mathrm{n}=0$ ) for the solution u of (12) and its derivative are given by

$$
\left\{\begin{array}{l}
u(t ; \varepsilon)=w_{0}(t)+0(\varepsilon), \quad \varepsilon+0 \\
\frac{d u}{d t}(t ; \varepsilon)=\frac{d w_{0}}{d t}(t)+\left\{c_{2}-\frac{d w_{0}}{d t}(0)\right\} \exp \left[-a\left(0, w_{0}(0)\right) \frac{t}{\varepsilon}\right]+0(\varepsilon), \quad \varepsilon \downarrow 0
\end{array}\right.
$$

uniformly valid in every bounded interval $0 \leq \mathrm{t} \leq \mathrm{T}<\mathrm{T}_{0}$, where $\mathrm{w}_{0}$ is the solution of the reduced problem (19).

COROLLARY 2. It follows from (32) and (26) that for any $\mathrm{n} \in \mathbb{I N} \cup\{0\}$

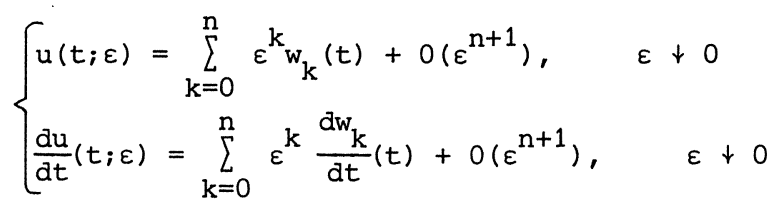

uniformly valid in every bounded interval $0<\delta \leq t \leq \mathrm{T}<\mathrm{T}_{0}$, where $\delta$ is an arbitrarily small positive constant independent of $\varepsilon$. 
REMARK 1. The statements of theorem 3 remain valid if the conditions (13) and (14) are replaced by the less stringent requirements

$$
\left\{\begin{array}{l}
a(t, u) \in C^{\infty}\left(\left[0, T_{1}\right] \times I\right), b(t, u) \in C^{\infty}\left(\left[0, T_{1}\right] \times I\right) \\
a(t, u)>0 \text { for } 0 \leq t \leq T_{1} \text { and } u \in I
\end{array}\right.
$$

where $T_{1}$ is some positive constant with $T_{1}<T_{0}$ and $I$ is some open subset of $\mathbb{R}$ containing the range $R_{w_{0}}$ of $w_{0}$ :

$$
I \supset \mathrm{R}_{\mathrm{w}_{0}}=\left\{\left.\mathrm{y} \in \mathbb{R}\right|_{t \in\left[0, \mathrm{~T}_{1}\right]} ^{\exists} \mathrm{y}=\mathrm{w}_{0}(t)\right\} .
$$

In that case the relations (32) are uniformly valid in every bounded interval $0 \leq t \leq T$ with $T \leq T_{1}$.

The conditions (33) have the advantage that they allow the functions $a(t, u)$ and $b(t, u)$ to have singularities in $u$, provided that these singulari.ties do not belong to $R_{w_{0}}$. As an illustration we consider the nonlinear initial value problem

$$
\left\{\begin{array}{l}
\varepsilon \frac{d^{2} u}{d t^{2}}+\frac{1}{u} \frac{d u}{d t}+u=0, \quad 0<t<\infty \\
u(0 ; \varepsilon)=C_{1}>0, \quad u_{t}(0 ; \varepsilon)=C_{2} .
\end{array}\right.
$$

It is easily established that

$$
w_{0}(t)=\frac{C_{1}}{1+C_{1} t} \quad \text { for } 0 \leq t<\infty
$$

and also that

$$
\frac{C_{1}}{1+C_{1} T_{1}} \leq w_{0}(t) \leq C_{1}
$$

valid for $0 \leq t \leq \mathrm{T}_{1}$, where $\mathrm{T}_{1}$ is an arbitrarily large positive constant. Hence it follows that the conditions (33) can be fulfilled by choosing

$$
I=\left\{u \in \mathbb{R} \mid \frac{C_{1}}{2\left(1+C_{1} T_{1}\right)}<u<C_{1}+1\right\},
$$

and we obtain 


$$
u(t ; \varepsilon)=\frac{C_{1}}{1+C_{1} t}+O(\varepsilon), \quad \varepsilon+0
$$

uniformly valid in every bounded interval $0 \leq t \leq \mathrm{T}$.

REMARK 2. The condition of infinite smoothness of the data can be weakened. In that case the expansions (32) are valid for all $\mathrm{n} \in \mathbb{I N} \cup\{0\}$ with $\mathrm{n} \leq \mathrm{n}_{0}$, the bound $n_{0}$ being determined by the differentiability of the data.

REMARK 3. Since the solution $u$ of an initial value problem which is governed by a differential equation of the type

$$
\frac{d u}{d t}=F(t, u)
$$

(with $\mathrm{u}$ and $\mathrm{F}$ vector-valued functions) is uniquely determined if $\mathrm{F}$ is continuous with respect to $t$ and locally lipschitzian with respect to $u$ (PICARD-LINDELÖF theorem), we may conclude that the solution $u$ of which the existence has been established in theorem 3 is unique. A similar remark can be made for the solution $u$ mentioned in theorem 2 . 
INITIAL VALUE PROBLEMS FOR LINEAR HYPERBOLIC DIFFERENTIAL EQUATIONS

In this chapter we shall investigate the initial value problem (singular perturbation problem)

1)

$$
\begin{cases}\varepsilon L_{2}[u]+L_{1}^{(\varepsilon)}[u]=f(x, t ; \varepsilon), \quad(x, t) \in D \\ u(x, 0 ; \varepsilon)=g(x ; \varepsilon), & -\infty<x<\infty \\ u_{t}(x, 0 ; \varepsilon)=h(x ; \varepsilon), & -\infty<x<\infty\end{cases}
$$

where $\varepsilon$ is a small positive parameter, i.e. $0<\varepsilon<1$, the region $D$ is the upper half-plane

$$
D=\{(x, t) \mid-\infty<x<\infty, 0<t<\infty\}
$$

and the differential operators $L_{2}$ and $L_{1}^{(\varepsilon)}$ are defined by

$$
L_{2}[u]=\frac{\partial^{2} u}{\partial t^{2}}+\left\{c_{1}(x, t)+c_{2}(x, t)\right\} \frac{\partial^{2} u}{\partial x \partial t}+c_{1}(x, t) c_{2}(x, t) \frac{\partial^{2} u}{\partial x^{2}}
$$

respectively

$$
L_{1}^{(\varepsilon)}[u]=a(x, t ; \varepsilon) \frac{\partial u}{\partial t}+b(x, t ; \varepsilon) \frac{\partial u}{\partial x}+d(x, t ; \varepsilon) u .
$$

The functions $a, b, d, f, g$ and $h$ depend on the variables $x$ and/or $t$ and on the parameter $\varepsilon$; the functions $c_{1}$ and $c_{2}$ satisfy

$$
c_{1}(x, t)<c_{2}(x, t) \text { in } \bar{D},
$$

which implies that $\mathrm{L}_{2}$ is a hyperbolic operator. 
Furthermore it will be assumed that the coefficient $a(x, t ; \varepsilon)$ is strictly positive, i.e.

$$
a(x, t ; \varepsilon) \geq a_{0}>0 \quad \text { in } \Omega
$$

valid for each compact subset $\Omega \subset \bar{D}$ provided that $\varepsilon$ is sufficiently small, say $0<\varepsilon \leq \varepsilon_{0}$. Here $a_{0}$ is some positive constant depending on $\Omega$ and on $\varepsilon_{0}$ but independent of $\varepsilon$.

Compared to singular perturbations of elliptic type there exists relatively few literature on singular perturbations of hyperbolic type. BLONDEL [3], [4], DZAVADOV [7], DE JAGER [26], SMITH [44] and SMITH \& PALMER [46] studied singularly perturbed initial value problems for linear hyperbolic differential equations, whereas singularly perturbed initial-boundary value problems were investigated by DZAVADOV [8] and GENET \& PUPION [18] (linear problems) and by GENET \& MADAUNE [17] and MADAUNE [30] (nonlinear problems). Singular perturbations of hyperbolic type are also discussed in the books by COLE [6, pp.129-140], ROSEAU [41, chapter 6] and WHITHAM [55, chapters 3 and 10]. The energy integral method that will be applied in this chapter to linear initial value problems of the type (1) is an extension of a similar method developed by DE JAGER [26] and differs basically from the methods used in $[3],[4],[7],[44]$ and [46]. A brief discussion of these papers together with a comparison of the results will be given in section 4 .

Section 1 of this chapter will be devoted to some preliminaries. In section 2 we shall derive a priori estimates for the solution $u$ of (1) and in section 3 we shall construct an asymptotic expansion into powers of $\varepsilon$ for this solution.

\section{Preliminaries}

We bring into mind that the characteristics of the operator $\mathrm{L}_{2}$ defined by (2) follow from the equations

$$
\frac{d x}{d t}=c_{1}(x, t) \quad \text { and } \quad \frac{d x}{d t}=c_{2}(x, t) .
$$

We shall assume that $c_{1}$ and $c_{2}$ are $c^{1}$-functions. Under this condition it is possible to draw at each point $\left(x_{0}, t_{0}\right)$ two vectors, tangent to the 
characteristics through $\left(x_{0}, t_{0}\right)$ and pointing in the direction of increasing $t$ (see figure 1). These vectors define the so-called characteristic directions at $\left(x_{0}, t_{0}\right)$.

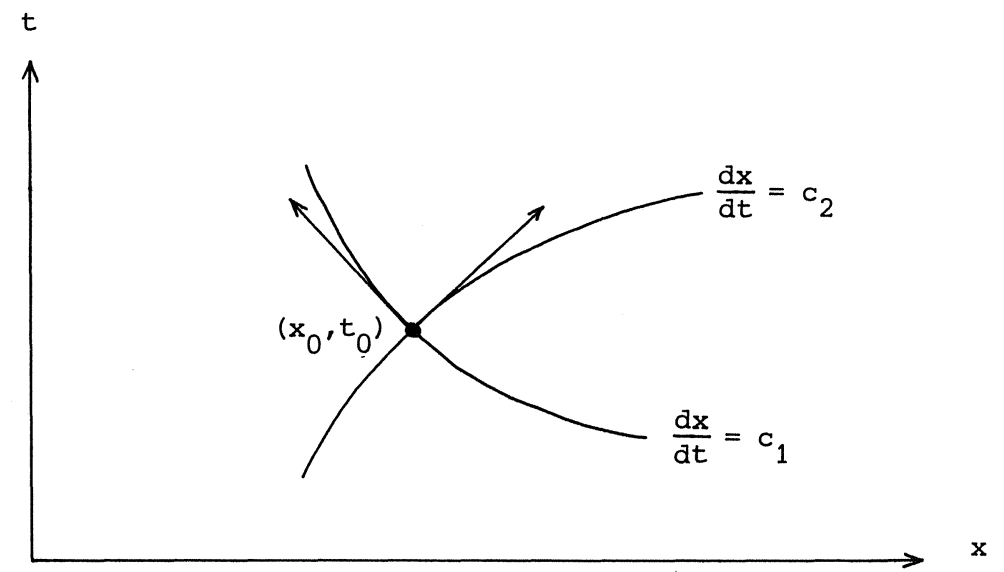

\section{Figure 1: Characteristic directions}

The characteristics of the operator $\mathrm{L}_{1}^{(\varepsilon)}$ defined by (3) follow from the equation

$$
\frac{d x}{d t}=\frac{b(x, t ; \varepsilon)}{a(x, t ; \varepsilon)}
$$

In order not to mix up the characteristics of $L_{2}$ and $L_{1}^{(\varepsilon)}$ we shall refer to the latter as subcharacteristics.

In the analysis of the initial value problem (1) the position of the subcharacteristics of the unperturbed operator $L_{1}(\varepsilon)$ with respect to the characteristics of the perturbing operator $\mathrm{L}_{2}$ plays an important role. Before entering into this subject we shall give a definition.

DEFINITION 1. Let $\phi(x, t)$ be a $C^{1}$-function satisfying $\phi_{x}^{2}+\phi_{t}^{2}>0$. The curve $\phi(x, t)=0$ has at one of its points $\left(x_{0}, t_{0}\right)$ a spacelike (resp. timelike resp. characteristic) direction with respect to the characteristic directions connected with $\mathrm{L}_{2}$ iff 


$$
\left(\phi_{t}+c_{1} \phi_{x}\right)\left(\phi_{t}+c_{2} \phi_{x}\right)>0 \quad(\text { resp. }<0 \text { resp. }=0) \text { at }\left(x_{0}, t_{0}\right)
$$

The curve $\phi(x, t)=0$ is said to be spacelike (resp. timelike resp. characteristic) if it has a spacelike (resp. timelike resp. characteristic) direction at each of its points.

This definition admits the following geometrical interpretation. Let the characteristic directions be described by the vectors $\vec{p}=\left(\begin{array}{c}C_{1} \\ 1\end{array}\right)$ and $\vec{q}=\left(\begin{array}{c}c_{2} \\ 1\end{array}\right)$. The direction of the tangent to the curve $\phi(x, t)=0$ can be characterized by a vector $\vec{r}=\left(\begin{array}{c}\phi_{t} \\ -\phi_{X}\end{array}\right)$. Evaluating cross products we obtain

$$
(\vec{r} \times \vec{p}) \cdot(\vec{r} \times \vec{q})=\left(\phi_{t}+c_{1} \phi_{x}\right)\left(\phi_{t}+c_{2} \phi_{x}\right)
$$

the dot denoting the scalar product.

Hence it follows that the curve $\phi(x, t)=0$ has a spacelike (resp. timelike) direction at $\left(x_{0}, t_{0}\right)$ iff the vectors $\vec{p}$ and $\vec{q}$ are lying on the same side (resp. on different sides) of the tangent to the curve at $\left(x_{0}, t_{0}\right)$ (see figure 2).

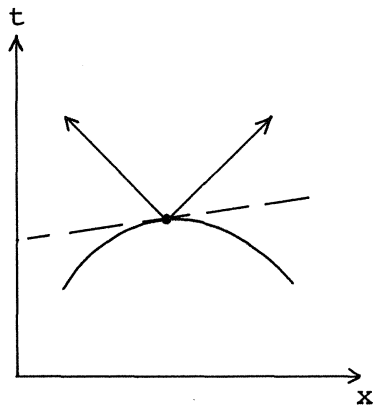

Spacelike

direction

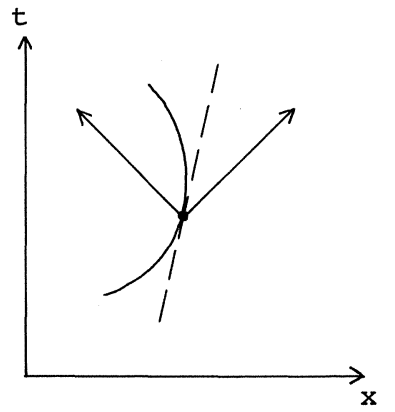

Timelike

direction

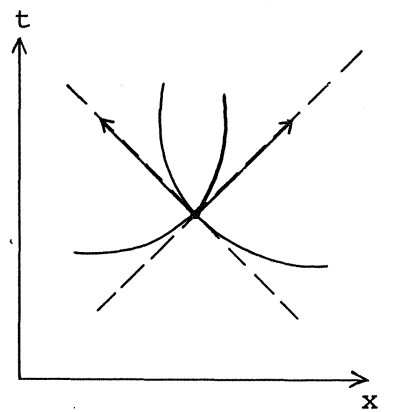

Characteristic

directions

Figure 2: Direction of a curve

With this knowledge we turn our attention to the following model problem (cf. COLE $[6, p .129]$ ) 
(4)

$$
\left\{\begin{array}{l}
\varepsilon\left\{\frac{\partial^{2} u}{\partial t^{2}}+\left(c_{1}+c_{2}\right) \frac{\partial^{2} u}{\partial x \partial t}+c_{1} c_{2} \frac{\partial^{2} u}{\partial x^{2}}\right\}+a \frac{\partial u}{\partial t}+b \frac{\partial u}{\partial x}=0, \quad(x, t) \in D \\
u(x, 0 ; \varepsilon)=g(x), \quad-\infty<x<\infty \\
u_{t}(x, 0 ; \varepsilon)=0, \quad-\infty<x<\infty
\end{array}\right.
$$

where $a, b, c_{1}$ and $c_{2}$ are constants with $a>0$ and $c_{1}<c_{2}$ and $g$ is a $c^{\infty}-$ function.

We shall investigate under which conditions the solution $w(x, t)$ of the reduced problem

$$
\begin{cases}a \frac{\partial w}{\partial t}+b \frac{\partial w}{\partial x}=0, & (x, t) \in D \\ w(x, 0)=g(x), & -\infty<x<\infty\end{cases}
$$

which is given by

$$
w(x, t)=g\left(x-\frac{b}{a} t\right)
$$

provides for small values of $\varepsilon$ an approximation for the solution $u(x, t ; \varepsilon)$ of (4).

We shall distinguish between problems with spacelike subcharacteristics and problems with timelike subcharacteristics.

1. The subcharacteristics are spacelike.

In this case, according to definition 1, the coefficients satisfy $\left(b-a c_{1}\right)\left(b-a c_{2}\right)>0$, that is

$$
\text { either } \frac{b}{a}<c_{1} \text { or } \frac{b}{a}>c_{2} \text {. }
$$

A possible situation is sketched in figure 3 . Note that the subcharacteristic through $P$ lies outside the characteristic triangle PRS. 


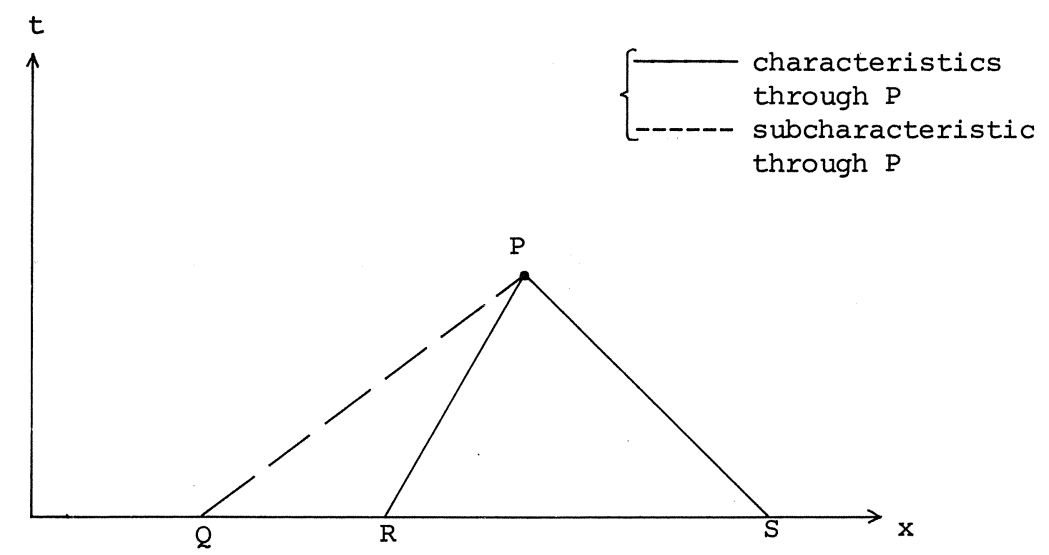

Figure 3: Spacelike subcharacteristics

From the theory of hyperbolic differential equations it is known (see for instance GARABEDIAN [16, p. 105]) that the value of the solution $u(x, t ; \varepsilon)$ at the point $P$ depends only on the values of $g(x)$ with $x$ in the segment RS. On the other hand the value of $w(x, t)$ at the point $P$ depends only on the value of $g$ at the point $Q$ (cf. (5)). Since the value of $g$ at $Q$ is independent of the values of $g(x)$ with $x$ in RS (in fact we assumed that $g(x)$ belongs to $C^{\infty}(I R)$, but not that $g(x)$ is analytic in IR) we expect that generally $w(x, t)$ does not approximate $u(x, t ; \varepsilon)$ for small values of $\varepsilon$. This is confirmed by the following example.

EXAMPLE 1. Consider problem (4) with $g(x)$ some $C^{\infty}$-function satisfying

$$
\left\{\begin{array}{l}
g(x)=x \text { for } x \leq-1 \\
g(x)=0 \text { for } x \geq 0 \\
-1<g(x)<0 \text { for }-1<x<0 .
\end{array}\right.
$$

The coefficients are supposed to satisfy $a>0, c_{1}<c_{2}$ and $0<c_{2}<\frac{b}{a}$, which implies that the subcharacteristics are spacelike.

It follows from the theory of hyperbolic differential equations that 


$$
u(x, t ; \varepsilon) \equiv 0 \text { for } 0 \leq c_{2} t \leq x<\infty \text {. }
$$

On the other hand the solution of the reduced problem satisfies

$$
w(x, t)=g\left(x-\frac{b}{a} t\right)<0 \text { for } 0<x<\frac{b}{a} t<\infty
$$

so the function $w(x, t)$ does not approximate $u(x, t ; \varepsilon)$ in the region $0<c_{2} t<x<\frac{b}{a} t<\infty$ (see figure 4).

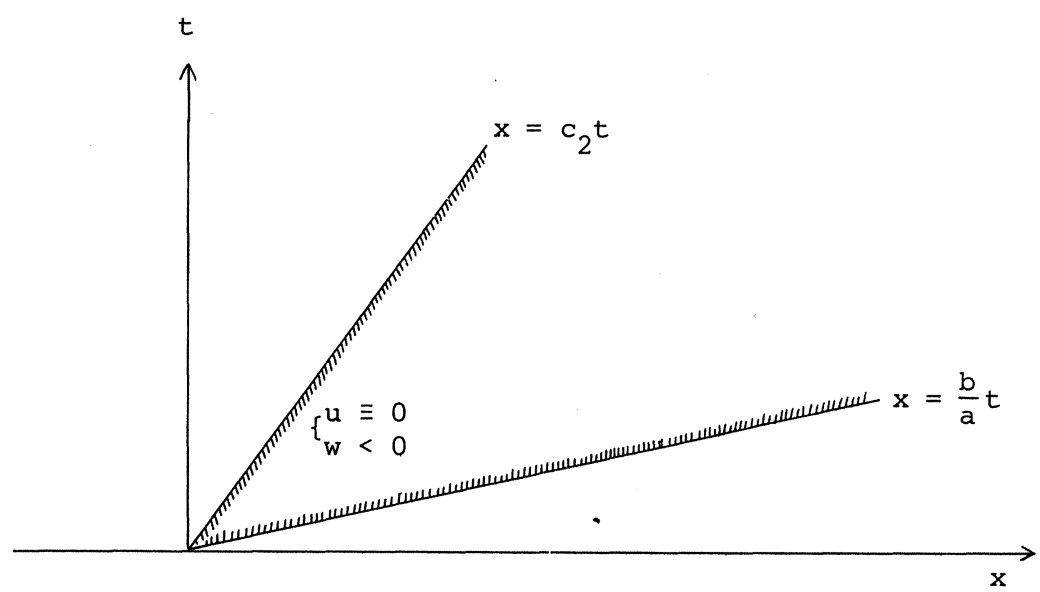

Figure 4

Hence we conclude that generally in the case of spacelike subcharacteristics the solution $w(x, t)$ of the reduced problem turns out to be a bad approximation for the solution $u(x, t ; \varepsilon)$ of $(4)$. However, $w(x, t)$ does not necessarily provide a bad approximation. This is illustrated by

EXAMPLE 2. Consider problem (4) with $g(x)=x$ and $a>0, c_{1}<c_{2}$. No other conditions are imposed on the coefficients.

It is easily verified that the solution of this problem is given by

$$
u(x, t ; \varepsilon)=x-\frac{b}{a} t+\varepsilon \frac{b}{a}\left\{1-\exp \left(-\frac{a t}{\varepsilon}\right)\right\} .
$$

Since $w(x, t)$ is given by

$$
w(x, t)=x-\frac{b}{a} t
$$


it follows that

$$
u(x, t ; \varepsilon)=w(x, t)+O(\varepsilon), \quad \varepsilon+0
$$

uniformly valid in $\bar{D}$, so in this example $w(x, t)$ approximates $u(x, t ; \varepsilon)$ for small values of $\varepsilon$, whether the subcharacteristics are spacelike or not. Note that if in this example the condition a $>0$ is replaced by a $<0$ the solution $u$ increases exponentially for all $(x, t)$ with $t \geq \delta>0$ if $\varepsilon$ tends to zero, regardless whether the subcharacteristics are timelike or spacelike. In this case $\mathrm{w}$ does not approximate $\mathrm{u}$ for small values of $\varepsilon$.

2. The subcharacteristics are timelike.

In this case the coefficients satisfy $\left(b-a c_{1}\right)\left(b-a c_{2}\right)<0$, that is

$$
c_{1}<\frac{b}{a}<c_{2}
$$

A typical situation is sketched in figure 5. Note that the subcharacteristic through $\mathrm{P}$ lies inside the characteristic triangle PRS.

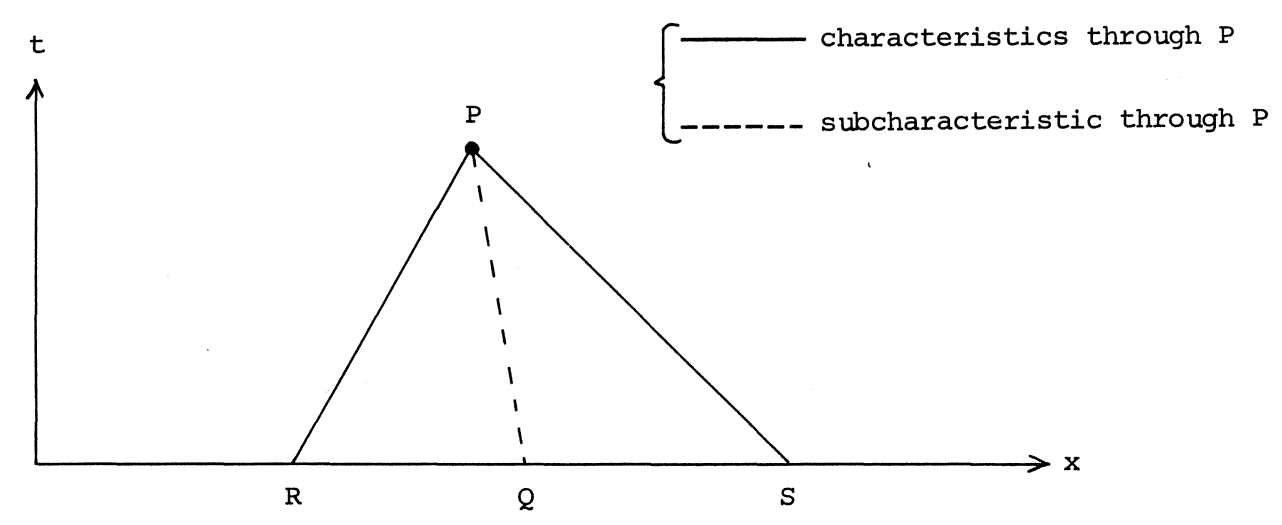

\section{Figure 5: Timelike subcharacteristics}

Using the same arguments as before (see text following figure 3 ) we have no reason to suspect $\mathrm{w}(\mathrm{x}, \mathrm{t})$ of being a bad approximation for $u(x, t ; \varepsilon)$. However, it follows from example 2 that the timelike condition (6) alone does not 
guarantee that $\mathrm{w}$ is a good approximation for $\mathrm{u}$; it appears that we have to impose an additional condition and by the results of example 2 we get the suggestion to impose $\mathrm{a}>0$ as additional condition. Therefore we shall confine ourselves from now on to problems with timelike subcharacteristics and a positive coefficient a. It will be shown in the sections 2 and 3 that these restrictions are sufficient in order that the solution $w$ of the reduced problem approximates the solution $u$ for small values of $\varepsilon$, not only for problems of type (4) with constant coefficients but also for more general problems of type (1). For a discussion of initial value problems with timelike subcharacteristics and a negative coefficient a we refer to BLONDEL [3], [4] (see also section 4).

We shall end this section with a physical interpretation of the conditions

$$
\mathrm{a}>0, \mathrm{c}_{1}<\frac{\mathrm{b}}{\mathrm{a}}<\mathrm{c}_{2} \text {. }
$$

Introducing characteristic coordinates by

$$
\left\{\begin{array}{l}
r=x-c_{1} t \\
s=x-c_{2} t
\end{array}\right.
$$

we may write the differential equation

$$
\varepsilon\left\{\frac{\partial^{2} u}{\partial t^{2}}+\left(c_{1}+c_{2}\right) \frac{\partial^{2} u}{\partial x \partial t}+c_{1} c_{2} \frac{\partial^{2} u}{\partial x^{2}}\right\}+a \frac{\partial u}{\partial t}+b \frac{\partial u}{\partial x}=0
$$

(with constant coefficients and $c_{1}<c_{2}$ ) in the form

$$
\varepsilon\left(c_{2}-c_{1}\right)^{2} \frac{\partial^{2} u}{\partial r \partial s}-\left(b-a c_{1}\right) \frac{\partial u}{\partial r}-\left(b-a c_{2}\right) \frac{\partial u}{\partial s}=0 .
$$

Let us consider a jump $k$ in the derivative $\frac{\partial u}{\partial s}$ across the characteristic $\mathbf{s}=\mathbf{s}_{0}:$

$$
\kappa=\kappa(r)=\frac{\partial u}{\partial s}\left(r, s_{0}+0\right)-\frac{\partial u}{\partial s}\left(r, s_{0}-0\right)
$$

We shall exanine the propagation of $\kappa$ along $s=s_{0}$.

Assuming that $u$ itself is continuous across $s=s_{0}$ we can evaluate 
equation (9) at $s=s_{0}+0$ and $s=s_{0}-0$ and form the difference to obtain

$$
\varepsilon\left(c_{2}-c_{1}\right)^{2} \frac{\partial \kappa}{\partial r}=\left(b-a c_{2}\right) k
$$

and hence

$$
\kappa=\kappa_{0} \exp \left[\frac{b-a c_{2}}{\varepsilon\left(c_{2}-c_{1}\right)^{2}}\left(r-r_{0}\right)\right] .
$$

Here $\kappa_{0}=\kappa\left(r_{0}\right)$ is the jump across $s=s_{0}$ at the point $\left(r_{0}, s_{0}\right)$. It follows that a jump across a characteristic propagates to infinity along that characteristic; in case $\mathrm{b}-\mathrm{ac}_{2}<0$ we have for $t \rightarrow \infty$ exponential decay, whereas for $b-a c_{2}>0$ we have exponential growth.

A similar discussion for jumps in $\frac{\partial u}{\partial r}$ across a characteristic $r=r_{0}$ shows that $b-a c_{1}>0$ implies exponential decay for $t \rightarrow \infty$, whereas $b-a c_{1}<0$ leads to exponential growth.

Hence we conclude that necessary and sufficient conditions for exponential decay are given by

$$
\left\{\begin{array}{l}
b-a c_{1}>0, \\
b-a c_{2}<0 .
\end{array}\right.
$$

Together with the condition $c_{1}<c_{2}$ these conditions are equivalent to

$$
a>0, c_{1}<\frac{b}{a}<c_{2} \text {. }
$$

Thus we have shown that the conditions (7) are in fact necessary and sufficient conditions for exponential decay of jumps in the first derivatives. We may expect these conditions to be fulfilled for any problem describing some physical system.

Another way to obtain the conditions (7) by physical arguments is the following (see also ROSEAU [41, p. 198]). Let us look for solutions of the equation (8) which are periodic in the space variable and exponentially decaying for $t \rightarrow \infty$. To this end we set 


$$
u=\exp (i k x-\lambda t)
$$

and we require that

$$
\begin{cases}\mathrm{k} \in \mathbb{R}, & \mathrm{k}>0 \\ \lambda \in \mathbb{C}, & \operatorname{Re} \lambda>0 .\end{cases}
$$

Substitution into the differential equation yields the following relation between $\lambda$ and $\mathrm{k}$

$$
f(\lambda)=\varepsilon\left(i k c_{1}-\lambda\right)\left(i k c_{2}-\lambda\right)+(i k b-a \lambda)=0 .
$$

The roots $\lambda$ of this equation will have a positive real part if and only if the quantity argf(iy) decreases by $2 \pi$ as $y$ increases from $-\infty$ to $+\infty$. For $y$ running through these values the point

$$
f(i y)=-\varepsilon\left(k c_{1}-y\right)\left(k c_{2}-y\right)+i(k b-a y)
$$

describes a parabola in the complex plane (see figure 6).

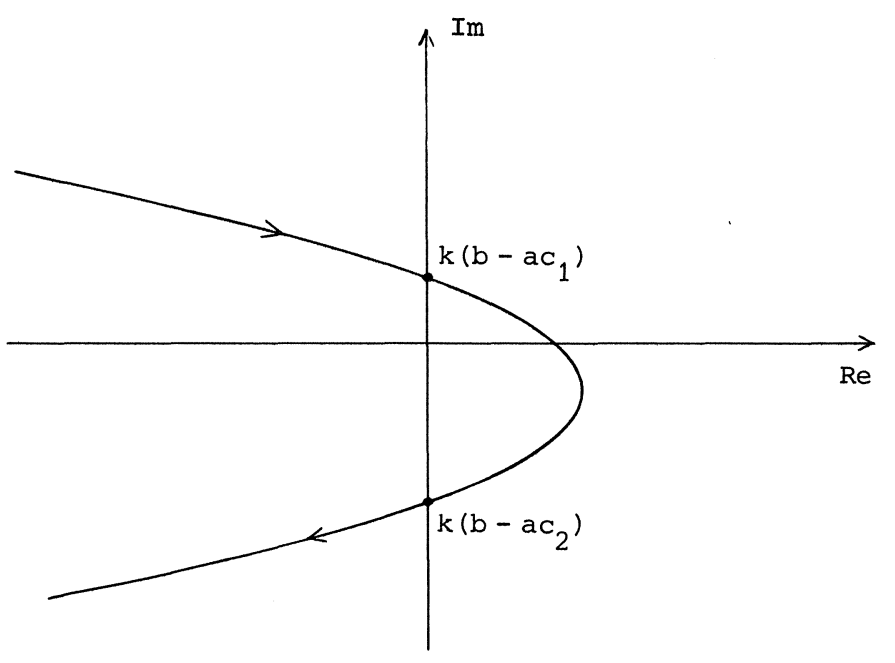

Figure 6: Position of $f(i y)$ 
It is easily verified that we have exponential decay if and only if a $>0$ (in which case the point $f(i y)$ travels in the proper direction along the parabola) and the origin lies inside the parabola. By calculating the points of intersection with the imaginary axis we see that the latter condition is equivalent to

$$
c_{1}<\frac{b}{a}<c_{2}
$$

indicating that the conditions (7) can also be considered as necessary and sufficient conditions for the equation (8) to admit exponentially decaying periodic solutions.

Now that we have established the physical relevancy of the conditions (7) we are going to derive in the sections 2 and 3 a priori estimates and asymptotic expansions for the solution of problem (1) under the conditions (7).

\section{A priori estimates}

We consider the initial value problem (1) under the following conditions:

(10)

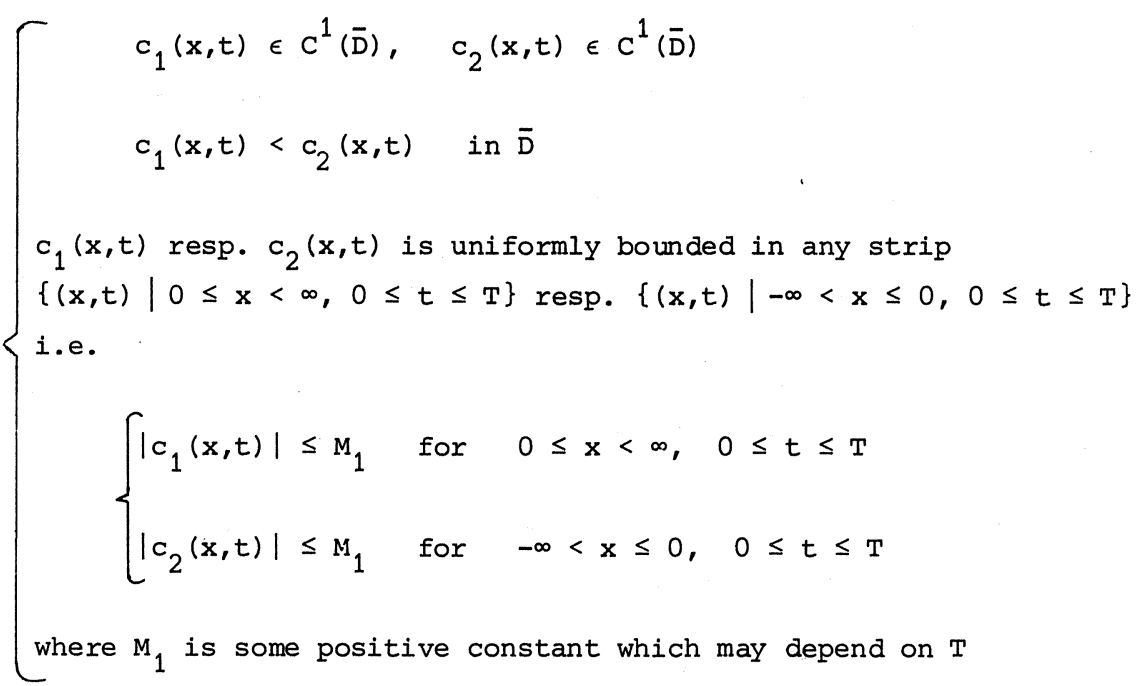




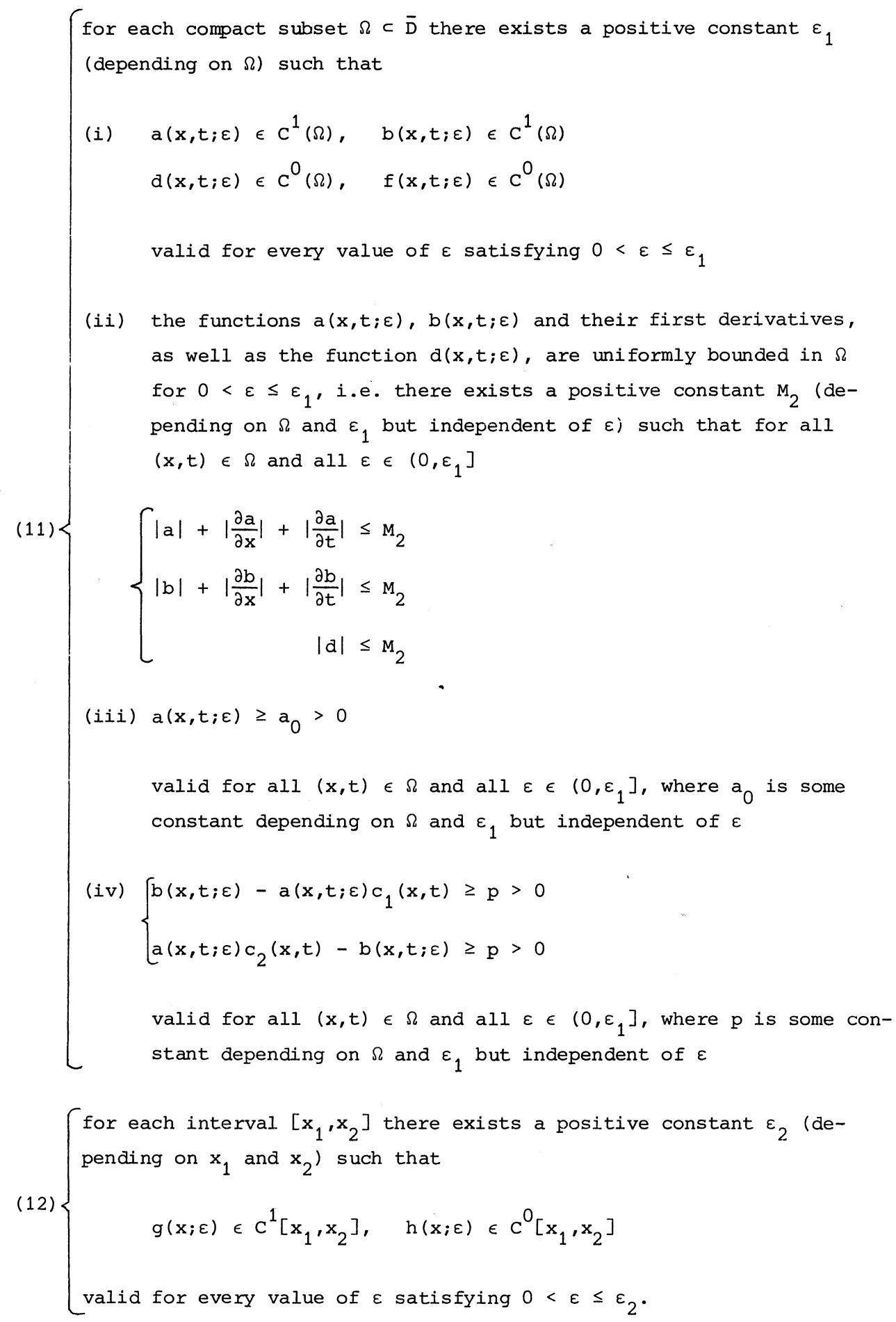


REMARK. The conditions $(11, i v)$ imply that the subcharacteristics of $L_{1}^{(\varepsilon)}$ are, for $\varepsilon$ sufficiently small, timelike with respect to the characteristic directions connected with $\mathrm{L}_{2}$.

In order to derive a priori estimates for the solution $u(x, t ; \varepsilon)$ of (1) in some compact subset $S \subset \bar{D}$ we enclose $S$ by a compact subset $\Omega \subset \bar{D}$ in the following way (see figure 7 ):

we draw the line $t=T$ where $T \geq \sup (x, t) \in S^{t}$.

Next we draw a characteristic $\left\{(x, t) \mid x=\gamma_{1}(t)\right\}$ satisfying $\frac{d x}{d t}=c_{1}$ and lying to the right of $S$. This characteristic intersects the lines $t=0$ resp. $t=T$ at the points $B$ resp. $C$.

Likewise we draw a characteristic $\left\{(x, t) \mid x=\gamma_{2}(t)\right\}$ to the left of $s$, satisfying $\frac{d x}{d t}=c_{2}$ and intersecting $t=0$ resp. $t=T$ at $A$ resp. D. It is assumed that $C$ lies to the right of $D$.

Then $\Omega$ is defined as the closure of the region $A B C D$.

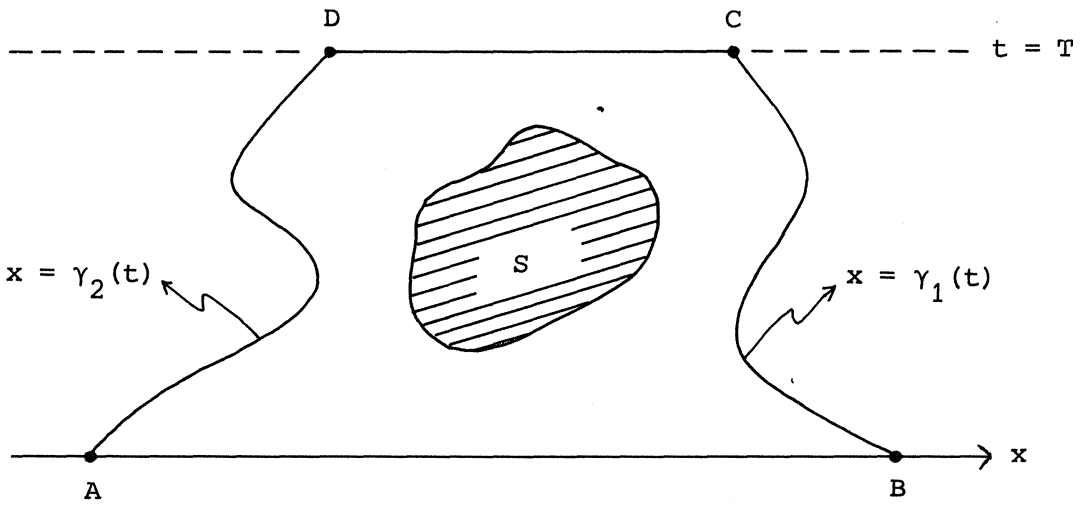

Figure 7: Construction of $\Omega$

Note that the construction of $\Omega$ is possible because of the boundedness of the functions $c_{1}$ and $c_{2}$, which has been postulated in (10).

Now that $\Omega$ has been determined we multiply the differential equation (1) by $2 \mathrm{u}$ to obtain (omitting arguments) 


$$
\begin{aligned}
& \frac{\partial}{\partial t}\left\{a u^{2}+2 \varepsilon u u_{t}\right\}+\frac{\partial}{\partial x}\left\{b u^{2}+2 \varepsilon\left(c_{1}+c_{2}\right) u_{t}+2 \varepsilon c_{1} c_{2} u_{x}\right\}= \\
& u^{2}\left\{-2 d+\frac{\partial a}{\partial t}+\frac{\partial b}{\partial x}\right\}+2 \varepsilon u_{t}^{2}+2 \varepsilon\left(c_{1}+c_{2}\right) u_{t} u_{x}+2 \varepsilon c_{1} c_{2} u_{x}^{2} \\
& +2 \varepsilon u u_{t} \frac{\partial\left(c_{1}+c_{2}\right)}{\partial x}+2 \varepsilon u u_{x} \frac{\partial\left(c_{1} c_{2}\right)}{\partial x}+2 f u
\end{aligned}
$$

valid for $(x, t) \in \Omega$ and $0<\varepsilon \leq \varepsilon_{0}=\min \left(\varepsilon_{1}, \varepsilon_{2}\right)$, the values of $\varepsilon_{1}$ and $\varepsilon_{2}$ depending on $\Omega$ (see the conditions $(11, i)$ and $(12)$ ).

Multiplication of (1) by $2 a u_{t}$ yields

$$
\begin{aligned}
& \frac{\partial}{\partial t}\left\{\varepsilon a u_{t}^{2}-\varepsilon a c_{1} c_{2} u_{x}^{2}\right\}+\frac{\partial}{\partial x}\left\{\varepsilon a\left(c_{1}+c_{2}\right) u_{t}^{2}+2 \varepsilon a c_{1} c_{2} u_{t} u_{x}\right\}= \\
& \varepsilon u_{t}^{2}\left\{\frac{\partial a}{\partial t}+\frac{\partial a\left(c_{1}+c_{2}\right)}{\partial x}\right\}+2 \varepsilon u_{t} u_{x} \frac{\partial\left(a c_{1} c_{2}\right)}{\partial x}-\varepsilon u_{x}^{2} \frac{\partial\left(a c_{1} c_{2}\right)}{\partial t} \\
& -2 a d u u_{t}-2 a^{2} u_{t}^{2}-2 a b u_{t} u_{x}+2 a f u_{t}
\end{aligned}
$$

valid for $(x, t) \in \Omega$ and $0<\varepsilon \leq \varepsilon_{0}$.

Finally, multiplication of (1) by $2 b_{x}$ leads to

$$
\begin{aligned}
& \frac{\partial}{\partial t}\left\{\varepsilon b\left(c_{1}+c_{2}\right) u_{x}^{2}+2 \varepsilon b u_{t} u_{x}\right\}-\frac{\partial}{\partial x}\left\{\varepsilon b u_{t}^{2}-\varepsilon b c_{1} c_{2} u_{x}^{2}\right\}= \\
& -\varepsilon u_{t}^{2} \frac{\partial b}{\partial x}+2 \varepsilon u_{t} u_{x} \frac{\partial b}{\partial t}+\varepsilon u_{x}^{2}\left\{\frac{\partial b\left(c_{1}+c_{2}\right)}{\partial t}+\frac{\partial\left(b c_{1} c_{2}\right)}{\partial x}\right\} \\
& -2 b d u u_{x}-2 a b u_{t} u_{x}-2 b^{2} u_{x}^{2}+2 b f u_{x}
\end{aligned}
$$

likewise valid for $(x, t) \in \Omega$ and $0<\varepsilon \leq \varepsilon_{0}$.

Adding (13), (14) and (15) and using the inequalities

$$
\begin{aligned}
& -2 a d u u_{t}-2 b d u u_{x}-2 a^{2} u_{t}^{2}-4 a b u_{t} u_{x}-2 b^{2} u_{x}^{2}+2 f u+2 a f u_{t}+2 b f u_{x}= \\
& -2\left(a u_{t}+b u_{x}\right)^{2}-2 d u\left(a u_{t}+b u_{x}\right)+2 f\left(a u_{t}+b u_{x}\right)+2 f u \leq \\
& \left(1+d^{2}\right) u^{2}+2 f^{2}
\end{aligned}
$$


and

$$
\begin{aligned}
& 2 \varepsilon u_{t} \frac{\partial\left(c_{1}+c_{2}\right)}{\partial x}+2 \varepsilon u u_{x} \frac{\partial\left(c_{1} c_{2}\right)}{\partial x} \leq \\
& 2 \varepsilon u^{2}+\varepsilon u_{t}^{2}\left\{\frac{\partial\left(c_{1}+c_{2}\right)}{\partial x}\right\}^{2}+\varepsilon u_{x}^{2}\left\{\frac{\partial\left(c_{1} c_{2}\right)}{\partial x}\right\}^{2} \leq \\
& 2 u^{2}+\varepsilon u_{t}^{2}\left\{\frac{\partial\left(c_{1}+c_{2}\right)}{\partial x}\right\}^{2}+\varepsilon u_{x}^{2}\left\{\frac{\partial\left(c_{1} c_{2}\right)}{\partial x}\right\}^{2}
\end{aligned}
$$

we obtain the relation

$$
\frac{\partial}{\partial t} Q_{1}+\frac{\partial}{\partial x} Q_{2} \leq Q_{3}+2 \dot{f}^{2}
$$

valid for $(x, t) \in \Omega$ and $0<\varepsilon \leq \varepsilon_{0}$, where the expressions $Q_{1}, Q_{2}$ and $Q_{3}$ (quadratic in $u, u_{t}$ and $u_{x}$ ) are defined by

$$
\left\{\begin{array}{c}
Q_{1}=a u^{2}+2 \varepsilon u u_{t}+\varepsilon a u_{t}^{2}+2 \varepsilon b u_{t} u_{x}+\varepsilon\left\{b\left(c_{1}+c_{2}\right)-a c_{1} c_{2}\right\} u_{x}^{2} \\
Q_{2}=b u^{2}+2 \varepsilon\left(c_{1}+c_{2}\right) u_{t}+2 \varepsilon c_{1} c_{2} u u_{x} \\
\quad+\varepsilon\left\{a\left(c_{1}+c_{2}\right)-b\right\} u_{t}^{2}+2 \varepsilon a c_{1} c_{2} u_{t} u_{x}+\varepsilon b c_{1} c_{2} u_{x}^{2} \\
Q_{3}=\gamma_{11} u^{2}+\varepsilon \gamma_{22} u_{t}^{2}+2 \varepsilon \gamma_{23} u_{t} u_{x}+\varepsilon \gamma_{33} u_{x}^{2}
\end{array}\right.
$$

with

$$
\left\{\begin{array}{l}
\gamma_{11}=3-2 d+d^{2}+\frac{\partial a}{\partial t}+\frac{\partial b}{\partial x} \\
\gamma_{22}=2+\frac{\partial a}{\partial t}-\frac{\partial b}{\partial x}+\frac{\partial a\left(c_{1}+c_{2}\right)}{\partial x}+\left\{\frac{\partial\left(c_{1}+c_{2}\right)}{\partial x}\right\}^{2} \\
\gamma_{23}=c_{1}+c_{2}+\frac{\partial b}{\partial t}+\frac{\partial\left(a c_{1} c_{2}\right)}{\partial x} \\
\gamma_{33}=2 c_{1} c_{2}+\frac{\partial b\left(c_{1}+c_{2}\right)}{\partial t}-\frac{\partial\left(a c_{1} c_{2}\right)}{\partial t}+\frac{\partial\left(b c_{1} c_{2}\right)}{\partial x}+\left\{\frac{\partial\left(c_{1} c_{2}\right)}{\partial x}\right\}^{2} .
\end{array}\right.
$$

Integrating (16) over $\Omega$ and using GREEN's theorem in the plane we obtain (see figure 7) 


$$
\int_{D}^{C} Q_{1} d x+\int_{B}^{C}\left(Q_{2}-c_{1} Q_{1}\right) d t+\int_{A}^{D}\left(c_{2} Q_{1}-Q_{2}\right) d t \leq
$$

$$
\iint_{\Omega} Q_{3} d x d t+2 \iint_{\Omega} f^{2} d x d t+\int_{A}^{B} Q_{1} d x
$$

valid for $0<\varepsilon \leq \varepsilon_{0}$.

In order to obtain a priori estimates for $u_{,} u_{x}$ and $u_{t}$ in $\Omega$ it is important that for $\varepsilon$ sufficiently small the following crucial requirements are fulfilled:

(i) the expression $Q_{1}$, quadratic in $u_{,} u_{t}$ and $u_{x^{\prime}}$ is positive definite in $\Omega$

(ii) the expression $\Omega_{2}-c_{1} Q_{1}$, quadratic in $u$ and $u_{t}+c_{1} u_{x}$, is positive definite in $\Omega$

(iii) the expression $c_{2} Q_{1}-Q_{2}$, quadratic in $u$ and $u_{t}+c_{2} u_{x}$, is positive definite in $\Omega$.

It is easily verified from (17) that (i) is fulfilled iff

$$
\left\{\begin{array}{l}
a>0 \quad \text { in } \Omega \\
\left(b-a c_{1}\right)\left(a c_{2}-b\right)>0 \quad \text { in } \Omega .
\end{array}\right.
$$

These conditions were supposed to be satisfied (see the conditions (11, iii and iv)).

REMARK. Note that these conditions are just the conditions (7) discussed at the end of section 1, which follow here in a natural way from the mathematical analysis.

Since we have for $\varepsilon$ sufficiently small

$$
b\left(c_{1}+c_{2}\right)-a c_{1} c_{2}=\frac{b^{2}+\left(b-a c_{1}\right)\left(a c_{2}-b\right)}{a} \geq \frac{b^{2}+p^{2}}{a}>0 \quad \text { in } \Omega
$$

it follows that 
(19)

$$
\begin{aligned}
& Q_{1}=a u^{2}+2 \varepsilon u u_{t}+\varepsilon a u_{t}^{2}+2 \varepsilon b u_{t} u_{x}+\varepsilon\left\{b\left(c_{1}+c_{2}\right)-a c_{1} c_{2}\right\} u_{x}^{2} \\
& \geq\left(a-\varepsilon^{\frac{1}{2}}\right) u^{2}+\varepsilon\left\{a-\frac{b^{2}+\frac{1}{2} p^{2}}{b\left(c_{1}+c_{2}\right)-a c_{1} c_{2}}-\varepsilon^{\frac{1}{2}}\right\} u_{t}^{2} \\
&+\varepsilon\left(1-\frac{b^{2}}{b^{2}+\frac{1}{2} p^{2}}\right)\left\{b\left(c_{1}+c_{2}\right)-a c_{1} c_{2}\right\} u_{x}^{2} \\
& \geq\left(a-\varepsilon^{\left.\frac{1}{2}\right) u^{2}}+\varepsilon\left(\frac{1}{2} \frac{a p^{2}}{b^{2}+p^{2}}-\varepsilon^{\frac{1}{2}}\right) u_{t}^{2}+\frac{1}{2} \varepsilon \frac{p^{2}}{a} u_{x}^{2}\right. \\
& \geq \frac{1}{2} a_{0} u^{2}+\frac{1}{4} \varepsilon \frac{a_{0} p^{2}}{b^{2}+p^{2}} u_{t}^{2}+\frac{1}{2} \varepsilon \frac{p^{2}}{a} u_{x}^{2} .
\end{aligned}
$$

It is easily established from (17) that

$$
q_{2}-c_{1} Q_{1}=\left(b-a c_{1}\right) u^{2}+2 \varepsilon c_{2} u\left(u_{t}+c_{1} u_{x}\right)+\varepsilon\left(a c_{2}-b\right)\left(u_{t}+c_{1} u_{x}\right)^{2}
$$

so we find for $\varepsilon$ sufficiently small

$$
Q_{2}-c_{1} Q_{1} \geq \frac{1}{2} p u^{2}+\frac{1}{2} \varepsilon p\left(u_{t}+c_{1} u_{x}\right)^{2}
$$

Similarly we obtain for $\varepsilon$ sufficiently small

$$
c_{2} Q_{1}-Q_{2} \geq \frac{1}{2} p u^{2}+\frac{1}{2} \varepsilon p\left(u_{t}+c_{2} u_{x}\right)^{2}
$$

Hence, using (19), (20) and (21) we arrive at

$$
\left\{\begin{array}{c}
Q_{1} \geq m\left(u^{2}+\varepsilon u_{t}^{2}+\varepsilon u_{x}^{2}\right) \\
Q_{2}-c_{1} Q_{1} \geq m\left\{u^{2}+\varepsilon\left(u_{t}+c_{1} u_{x}\right)^{2}\right\} \\
c_{2} Q_{1}-Q_{2} \geq m\left\{u^{2}+\varepsilon\left(u_{t}+c_{2} u_{x}\right)^{2}\right\}
\end{array}\right.
$$

valid for $(x, t) \in \Omega$ and $\varepsilon$ sufficiently small, say $0<\varepsilon \leq \varepsilon_{0}$, where $\varepsilon_{0}$ is some generic constant and the positive constant $\mathrm{m}$, depending on $\Omega$ but independent of $\varepsilon$, is given by

$$
m=\frac{1}{4} p \min \left(2, \frac{a_{0} p}{p^{2}+m_{2}^{2}}, \frac{2 p}{M_{2}}\right) .
$$


It is easily established that there exists a constant $M \geq 2$, depending on $\Omega$ but independent of $\varepsilon$, such that

$$
\left|Q_{1}\right|+\left|Q_{3}\right| \leq M\left(u^{2}+\varepsilon u_{t}^{2}+\varepsilon u_{x}^{2}\right)
$$

valid for $(x, t) \in \Omega$ and $0<\varepsilon \leq \varepsilon_{0}$.

Using (22) and (23) we obtain from (18)

$$
\begin{aligned}
m \int_{D}^{C}\left(u^{2}+\varepsilon u_{t}^{2}+\varepsilon u_{x}^{2}\right) d x & +m \int_{B}^{C}\left\{u^{2}+\varepsilon\left(u_{t}+c_{1} u_{x}\right)^{2}\right\} d t \\
& +m \int_{A}^{D}\left\{u^{2}+\varepsilon\left(u_{t}+c_{2} u_{x}\right)^{2}\right\} d t \leq
\end{aligned}
$$$$
M \iint_{\Omega}\left(u^{2}+\varepsilon u_{t}^{2}+\varepsilon u_{x}^{2}\right) d x d t+M_{1}^{2}(\Omega, \varepsilon)
$$

valid for $0<\varepsilon \leq \varepsilon_{0}$, where the positive constant $\mathrm{K}_{1}(\Omega, \varepsilon)$, depending on $\Omega$ and $\varepsilon$, is defined by

$$
K_{1}(\Omega, \varepsilon)=\|f\|_{\Omega}+\|g\|_{I}+\varepsilon^{-\frac{1}{2}}\left\|_{g_{X}}\right\|_{I}+\varepsilon^{\frac{1}{2}}\left\|_{h}\right\|_{I^{\prime}}
$$

I denoting the segment $A B=\left[\gamma_{2}(0), \gamma_{1}(0)\right]$ (see figure 7 ).

One easily verifies that formula (24) with the same values of $m$ and $M$ is also valid for any similar region $\Omega^{\prime} \subset \Omega$ with $A^{\prime} B^{\prime} \subset A B, D^{\prime} C^{\prime} / / D C$ and $B^{\prime} C^{\prime}$ and $A^{\prime} D^{\prime}$ characteristic segments with directions $c_{1}$ resp. $c_{2}$ (see figure 8 ).

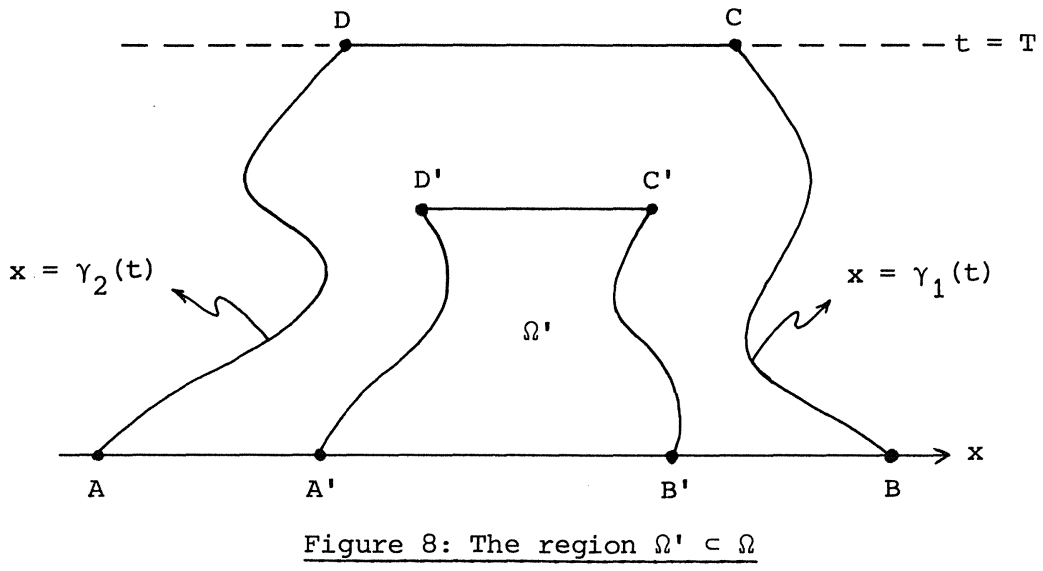


So applying (24) to regions $\Omega^{\prime} \subset \Omega$ with $A^{\prime}=A, B^{\prime}=B$ and $D^{\prime} C^{\prime} / / D C$ we obtain

$$
\int_{\gamma_{2}(t)}^{\gamma_{1}(t)}\left(u^{2}+\varepsilon u_{t}^{2}+\varepsilon u_{x}^{2}\right) d x-\frac{M}{m} \int_{0}^{t} \int_{\gamma_{2}(\tau)}^{\gamma_{1}(\tau)}\left(u^{2}+\varepsilon u_{t}^{2}+\varepsilon u_{x}^{2}\right) d x d \tau \leq \frac{M}{m} k_{1}^{2}(\Omega, \varepsilon)
$$

valid for $0 \leq t \leq T$ and $0<\varepsilon \leq \varepsilon_{0}$.

Using GRONWALL's lemma we derive from (25) the estimate

$$
\int_{\gamma_{2}(t)}^{\gamma_{1}(t)}\left(u^{2}+\varepsilon u_{t}^{2}+\varepsilon u_{x}^{2}\right) d x \leq C(\Omega) k_{1}^{2}(\Omega, \varepsilon)
$$

valid for $0 \leq t \leq T$ and $0<\varepsilon \leq \varepsilon_{0}$, where the positive constant $C(\Omega)$, depending on $\Omega$ but independent of $\varepsilon$, is given by

$$
C(\Omega)=\frac{M}{m} \exp \left(\frac{M T}{m}\right)
$$

Applying (24) to a region $\Omega^{\prime}$ inside $\Omega$ and using the estimate $(26)$ we obtain

$$
\left\{\begin{array}{l}
\int_{B^{\prime}}^{C^{\prime}}\left\{u^{2}+\varepsilon\left(u_{t}+c_{1} u_{x}\right)^{2}\right\} d t \leq^{-C}(\Omega) K_{1}^{2}(\Omega, \varepsilon) \\
\int_{A^{\prime}}^{D^{\prime}}\left\{u^{2}+\varepsilon\left(u_{t}+C_{2} u_{x}\right)^{2}\right\} d t \leq C(\Omega) K_{1}^{2}(\Omega, \varepsilon)
\end{array}\right.
$$

valid for all characteristic segments $B^{\prime} C^{\prime}$ and $A^{\prime} D^{\prime}$ iying inside $\Omega$ (with directions $c_{1}$ resp. $c_{2}$ ) and all $\varepsilon$ satisfying $0<\varepsilon \leq \varepsilon_{0}$. Here $C(\Omega)$ denotes some generic positive constant, depending on $\Omega$ but independent of $\varepsilon$.

Now that we have established estimates in $\mathrm{L}_{2}$-norm it is not difficult to derive pointwise estimates for $u$ and its first derivatives. Using (26) we obtain for every $(x, t) \in \Omega$ and $0<\varepsilon \leq \varepsilon_{0}$ 
(28)

$$
\begin{aligned}
& \left|u^{2}(x, t ; \varepsilon)-u^{2}\left(\gamma_{2}(t), t ; \varepsilon\right)\right|= \\
& \left|2 \int_{\gamma_{2}(t)}^{x} u(\xi, t ; \varepsilon) u_{x}(\xi, t ; \varepsilon) d \xi\right| \leq \\
& 2 \int_{\gamma_{1}(t)}\left|u(\xi, t ; \varepsilon) u_{x}(\xi, t ; \varepsilon)\right| d \xi \leq \\
& \left.2\left[\int_{\gamma_{2}(t)}^{(t)} u^{2}(\xi, t ; \varepsilon) d \xi\right]_{\gamma_{2}(t)}^{\gamma_{1}(t)} u_{x}^{2}(\xi, t ; \varepsilon) d \xi\right]^{\frac{1}{2}} \leq \\
& C(\Omega) \varepsilon^{-\frac{1}{2}} K_{1}^{2}(\Omega, \varepsilon)
\end{aligned}
$$

or

$$
u^{2}\left(\gamma_{2}(t), t ; \varepsilon\right) \leq u^{2}(x, t ; \varepsilon)+C(\Omega) \varepsilon^{-\frac{1}{2}} K_{1}^{2}(\Omega, \varepsilon)
$$

Integrating this inequality with respect to $x$ between the limits $\gamma_{2}(t)$ and $\gamma_{1}(t)$ we obtain

$$
\Theta_{1} u^{2}\left(\gamma_{2}(t), t ; \varepsilon\right) \leq C(\Omega) K_{1}^{2}(\Omega, \varepsilon)+\Theta_{2} C(\Omega) \varepsilon^{-\frac{1}{2}} K_{1}^{2}(\Omega)
$$

with

$$
\left\{\begin{array}{l}
\Theta_{1}=\inf _{[0, T]}^{\operatorname{Tn}}\left\{\gamma_{1}(t)-\gamma_{2}(t)\right\}>0 \\
\Theta_{2}=\sup _{[0, T]}\left\{\gamma_{1}(t)-\gamma_{2}(t)\right\}>0
\end{array}\right.
$$

so we derive from (28) and (29) the pointwise estimate

$$
|u(x, t ; \varepsilon)| \leq C(\Omega) \varepsilon^{-\frac{1}{4}} K_{1}(\Omega, \varepsilon)
$$

valid for $(x, t) \in \Omega$ and $0<\varepsilon \leq \varepsilon_{0}$, where $C(\Omega)$ is a positive constant depending on $\Omega$ but independent of $\varepsilon$, and $\varepsilon_{0}$ is some generic constant.

In order to obtain pointwise estimates for $\frac{\partial u}{\partial x}$ and $\frac{\partial u}{\partial t}$ in $\Omega$ we proceed as follows (see figure 9): 


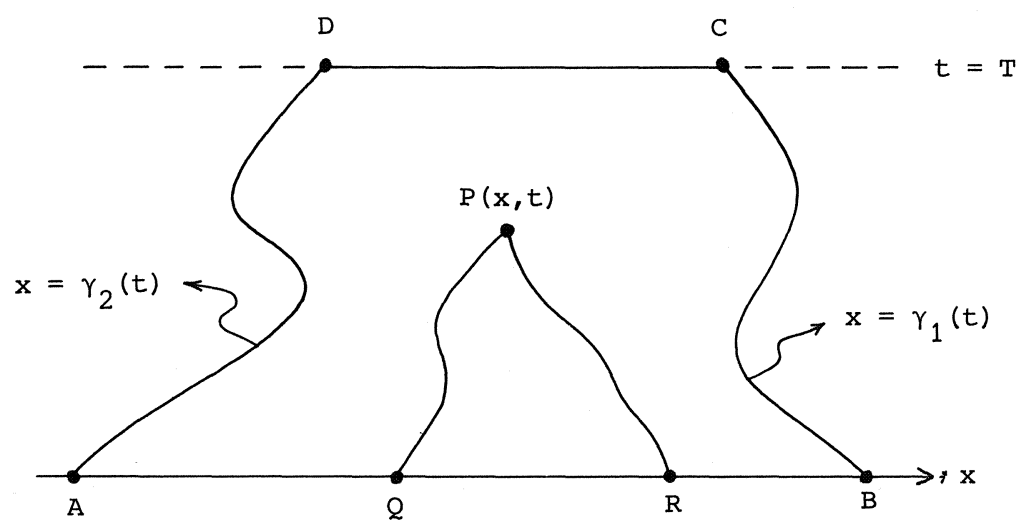

Figure 9

Let $\mathrm{P}(\mathrm{x}, \mathrm{t})$ be an arbitrary point in $\Omega$. We draw two characteristics through $P$, one with direction $c_{2}$ and intersecting the line $t=0$ at $Q$, the other with direction $c_{1}$ and intersecting the initial line at $R$.

We multiply the differential equation (1) by $2\left(u_{t}+c_{1} u_{x}\right)$ to obtain after some calculations

$$
\begin{aligned}
\varepsilon\left(\frac{\partial}{\partial t}+c_{2} \frac{\partial}{\partial x}\right)\left(u_{t}+c_{1} u_{x}\right)^{2}= & -\frac{2\left(a c_{2}-\tilde{b}\right)}{c_{2}-c_{1}}\left(u_{t}+c_{1} u_{x}\right)^{2} \\
& -\frac{2\left(\tilde{b}-a c_{1}\right)}{c_{2}-c_{1}}\left(u_{t}+c_{2} u_{x}\right)\left(u_{t}+c_{1} u_{x}\right) \\
& -2 d u\left(u_{t}+c_{1} u_{x}\right)+2 f\left(u_{t}+c_{1} u_{x}\right)
\end{aligned}
$$

valid for $(x, t) \in \Omega$ and $0<\varepsilon \leq \varepsilon_{0}$, where

$$
\tilde{b}=b-\varepsilon\left(\frac{\partial c_{1}}{\partial t}+c_{2} \frac{\partial c_{1}}{\partial x}\right) \text {. }
$$

From the timelike condition $(11, i v)$ we conclude that for $\varepsilon$ sufficiently small, say $0<\varepsilon \leq \varepsilon_{0}$ 


$$
a(x, t ; \varepsilon) c_{2}(x, t)-\tilde{b}(x, t ; \varepsilon) \geq \frac{1}{2} p \quad \text { in } \Omega
$$

Hence if follows from (30) that

$$
\begin{aligned}
& \varepsilon\left(\frac{\partial}{\partial t}+c_{2} \frac{\partial}{\partial x}\right)\left(u_{t}+c_{1} u_{x}\right)^{2} \leq \\
& -\frac{p}{c_{2}-c_{1}}\left(u_{t}+c_{1} u_{x}\right)^{2}-\frac{2}{c_{2}-c_{1}}\left(u_{t}+c_{1} u_{x}\right)\left(\tilde{b}-a_{1}\right)\left(u_{t}+c_{2} u_{x}\right) \\
& \frac{1}{p\left(c_{2}-c_{1}\right)}\left\{\left(\tilde{b}_{-a c_{1}}\right)\left(u_{t}+\frac{2}{c_{2}-c_{1}}\left(u_{t}+c_{1} u_{x}\right)+a\left(c_{2}-c_{1}\right) u-\left(c_{2}-c_{1}\right) f\right\} \leq\right. \\
& \frac{3\left(\tilde{b}_{-a c_{1}}\right)^{2}}{p\left(c_{2}-c_{1}\right)}\left(u_{t}+c_{2} u_{x}\right)^{2}+\frac{\left.3 c_{1}\right) u-\left(c_{2}-c_{1}\right)}{p} u^{2}+\frac{3\left(c_{2}-c_{1}\right)}{p} f^{2} \leq
\end{aligned}
$$

valid for $(x, t) \in \Omega$ and $0<\varepsilon \leq \varepsilon_{0}$.

Integrating this inequality with respect to $t$ along the characteristic $Q P$ and using the second estimate in (27) we obtain

$$
\left|\left(u_{t}+c_{1} u_{x}\right)(x, t ; \varepsilon)\right| \leq C(\Omega) \varepsilon^{-1} K_{2}(\Omega, \varepsilon)
$$

valid for $(\mathrm{x}, \mathrm{t}) \in \Omega$ and $0<\varepsilon \leq \varepsilon_{\mathrm{O}^{\prime}}$, where $\mathrm{C}(\Omega)$ is a positive constant (depending on $\Omega$ but independent of $\varepsilon$ ) and the positive constant $\mathrm{K}_{2}(\Omega, \varepsilon)$ (depending on both $\Omega$ and $\varepsilon$ ) is given by

$$
\mathrm{K}_{2}(\Omega, \varepsilon)=\sup _{\Omega}|f|+\|g\|_{I}+\varepsilon^{\frac{1}{2}} \sup _{I}\left|g_{\mathrm{x}}\right|+\varepsilon^{\frac{1}{2}} \sup _{I}|\mathrm{~h}| .
$$

Likewise, multiplying (1) by $2\left(u_{t}+c_{1} u_{x}\right)$ and integrating along the characteristic RP we obtain

$$
\left|\left(u_{t}+c_{2} u_{x}\right)(x, t ; \varepsilon)\right| \leq C(\Omega) \varepsilon^{-1} K_{2}(\Omega, \varepsilon)
$$

valid for $(x, t) \in \Omega$ and $0<\varepsilon \leq \varepsilon_{0}$.

Finally, it follows from (31) and (32) that 


$$
\left\{\begin{array}{l}
\left|\frac{\partial u}{\partial x}(x, t ; \varepsilon)\right| \leq C(\Omega) \varepsilon^{-1} K_{2}(\Omega, \varepsilon) \\
\left|\frac{\partial u}{\partial t}(x, t ; \varepsilon)\right| \leq C(\Omega) \varepsilon^{-1} K_{2}(\Omega, \varepsilon)
\end{array}\right.
$$

valid for $(x, t) \in \Omega$ and $0<\varepsilon \leq \varepsilon_{0}$.

We summarize the results in

THEOREM 1. Let $\mathrm{u}(\mathrm{x}, \mathrm{t} ; \varepsilon)$ be the solution of the initial value problem (1) under the conditions (10), (11) and (12), and let the region $\Omega$ be given as in figure 7.

Then we have for $\varepsilon$ sufficiently small, say $0<\varepsilon \leq \varepsilon_{0}$ with $\varepsilon_{0}$ depending on $\Omega$, the following estimates in $\mathrm{L}_{2}$-norm

$$
\left\{\begin{array}{l}
\int_{r_{2}(t)}^{r_{1}(t)}\left(u^{2}+\varepsilon u_{t}^{2}+\varepsilon u_{x}^{2}\right) d x \leq C(\Omega) k_{1}^{2}(\Omega, \varepsilon) \quad \text { valid for } 0 \leq t \leq T \\
\int_{B^{\prime}}^{C^{\prime}}\left\{u^{2}+\varepsilon\left(u_{t}+c_{1} u_{x}\right)^{2}\right\} d t \leq C(\Omega) k_{1}^{2}(\Omega, \varepsilon) \\
\int_{A^{\prime}}^{D^{\prime}}\left\{u^{2}+\varepsilon\left(u_{t}+c_{2} u_{x}\right)^{2}\right\} d t \leq C(\Omega) k_{1}^{2}(\Omega, \varepsilon)
\end{array}\right.
$$

where $\mathrm{B}^{\prime} \mathrm{C}^{\prime}$ and $\mathrm{A}^{\prime} \mathrm{D}^{\prime}$ are arbitrary characteristic segments in $\Omega$ with directions $\mathrm{c}_{1}$ resp. $\mathrm{c}_{2}$. The positive constant $\mathrm{C}(\Omega)$ depends on $\Omega$ but not on the parameter $\varepsilon$. The positive constant $\mathrm{K}_{1}(\Omega, \varepsilon)$, depending on both $\Omega$ and $\varepsilon$, is given by

$$
\mathrm{K}_{1}(\Omega, \varepsilon)=\|f\|_{\Omega}+\|\mathrm{g}\|_{I}+\varepsilon^{\frac{1}{2}}\left\|_{g_{X}}\right\|_{I}+\varepsilon^{\frac{1}{2}}\left\|_{h}\right\|_{I}
$$

with $I=\Omega \cap\{(x, t) \mid t=0\}$.

We also have for $\varepsilon$ sufficiently small the following pointwise estimates

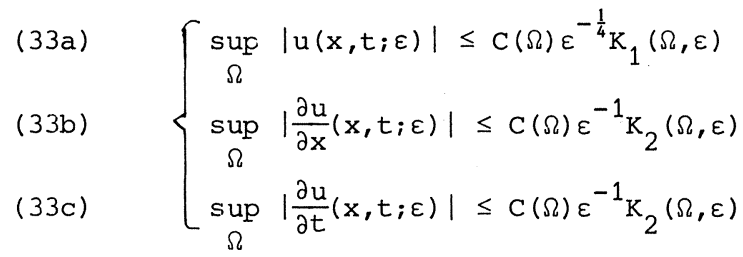


where again the positive constant $\mathrm{C}(\Omega)$ depends on $\Omega$ but not on $\varepsilon$. The positive constant $\mathrm{K}_{2}(\Omega, \varepsilon)$, depending on both $\Omega$ and $\varepsilon$, is given by

$$
\mathrm{K}_{2}(\Omega, \varepsilon)=\sup _{\Omega}|f|+\|g\|_{I}+\varepsilon^{\frac{1}{2}} \sup _{I}\left|g_{X}\right|+\varepsilon^{\frac{1}{2}} \sup _{I}|h| .
$$

\section{Asymptotic expansions}

As an application of the a priori estimates that we derived in the preceding section we shall now construct an asymptotic expansion into powers of $\varepsilon$ for the solution $u(x, t ; \varepsilon)$ of the initial value problem

$$
\left\{\begin{array}{l}
\varepsilon L_{2}[u]+L_{1}[u]=f(x, t), \quad(x, t) \in D \\
u(x, 0 ; \varepsilon)=g(x), \quad-\infty<x<\infty \\
u_{t}(x, 0 ; \varepsilon)=h(x), \quad-\infty<x<\infty
\end{array}\right.
$$

where $0<\varepsilon \ll 1$ and

$$
\left\{\begin{array}{l}
L_{2}[u]=\frac{\partial^{2} u}{\partial t^{2}}+\left\{c_{1}(x, t)+c_{2}(x, t)\right\} \frac{\partial^{2} u}{\partial x \partial t}+c_{1}(x, t) c_{2}(x, t) \frac{\partial^{2} u}{\partial x^{2}} \\
L_{1}[u]=a(x, t) \frac{\partial u}{\partial t}+b(x, t) \frac{\partial u}{\partial x}+d(x, t) u .
\end{array} .\right.
$$

In order not to complicate the calculations it will be assumed that all functions belong to the class $c^{\infty}$. In addition we shall require

(35) $\left\{\begin{array}{c}c_{1}(x, t)<c_{2}(x, t) \text { in } \bar{D} \\ c_{1}(x, t) \text { uniformly bounded in any strip }\{(x, t) \mid 0 \leq x<\infty, 0 \leq t \leq T\} \\ c_{2}(x, t) \text { uniformly bounded in any strip }\{(x, t) \mid-\infty<x \leq 0,0 \leq t \leq T\} \\ a(x, t)>0 \quad \text { in } \bar{D} \\ a(x, t) c_{1}(x, t)<b(x, t)<a(x, t) c_{2}(x, t) \quad \text { in } \bar{D} .\end{array}\right.$

The construction of the formal approximation is similar to the construction given in chapter I, section 2 for ordinary differential equations. Expanding the coefficients into a Taylor series we obtain for any $n \in \mathbb{N} \cup\{0\}$ 


$$
\left\{\begin{array}{l}
a(x, t)=\sum_{k=0}^{n} a_{k}(x) t^{k}+a_{n+1}(x, t) t^{n+1} \\
b(x, t)=\sum_{k=0}^{n} b_{k}(x) t^{k}+b_{n+1}(x, t) t^{n+1} \\
c_{1}(x, t)+c_{2}(x, t)=\sum_{k=0}^{n} \tilde{c}_{k}(x) t^{k}+\tilde{c}_{n+1}(x, t) t^{n+1} \\
c_{1}(x, t) c_{2}(x, t)=\sum_{k=0}^{n} c_{k}^{*}(x) t^{k}+c_{n+1}^{*}(x, t) t^{n+1} \\
d(x, t)=\sum_{k=0}^{n} a_{k}(x) t^{k}+d_{n+1}(x, t) t^{n+1}
\end{array}\right.
$$

where $a_{0}(x)=a(x, 0)$ etc.

We define the differential operator $L^{(\varepsilon)}$ by

$$
L^{(\varepsilon)}=\varepsilon L_{2}+L_{1}
$$

Putting $\tau=\frac{t}{\varepsilon}$ and expanding $L^{(\varepsilon)}$ into powers of $\varepsilon$ we obtain

$$
\begin{aligned}
& L^{(\varepsilon)}=\varepsilon^{-1} \frac{\partial^{2}}{\partial \tau^{2}}+\left\{\sum_{k=0}^{n} \tilde{c}_{k}(x) \varepsilon^{k} \tau^{k}+\tilde{c}_{n+1}(x, \varepsilon \tau) \varepsilon^{n+1} \tau^{n+1}\right\} \frac{\partial^{2}}{\partial x \partial \tau} \\
& +\varepsilon\left\{\sum_{k=0}^{n} c_{k}^{*}(x) \varepsilon^{k} \tau^{k}+c_{n+1}^{*}(x, \varepsilon \tau) \varepsilon^{n+1} \tau^{n+1}\right\} \frac{\partial^{2}}{\partial x^{2}} \\
& +\varepsilon^{-1}\left\{\sum_{k=0}^{n} a_{k}(x) \varepsilon^{k} \tau^{k}+a_{n+1}(x, \varepsilon \tau) \varepsilon^{n+1} \tau^{n+1}\right\} \frac{\partial}{\partial \tau} \\
& +\left\{\sum_{k=0}^{n} b_{k}(x) \varepsilon^{k} \tau^{k}+b_{n+1}(x, \varepsilon \tau) \varepsilon^{n+1} \tau^{n+1}\right\} \frac{\partial}{\partial x} \\
& +\left\{\sum_{k=0}^{n} d_{k}(x) \varepsilon^{k} \tau^{k}+d_{n+1}(x, \varepsilon \tau) \varepsilon^{n+1} \tau^{n+1}\right\} .
\end{aligned}
$$

We shall construct a formal approximation $\tilde{u}(x, t ; \varepsilon)$ of $u(x, t ; \varepsilon)$ in the form

$$
\tilde{u}(x, t ; \varepsilon)=\sum_{k=0}^{n} \varepsilon^{k} w_{k}(x, t)+\sum_{k=0}^{n} \varepsilon^{k+1} v_{k}(x, \tau)
$$


where the functions $w_{k}$ and $v_{k}(k=0,1, \ldots, n)$ have to be determined. A calculation shows that

$$
\begin{aligned}
L^{(\varepsilon)}[\tilde{u}]= & L^{(\varepsilon)}\left[\sum_{k=0}^{n} \varepsilon^{k} w_{k}\right]+L^{(\varepsilon)}\left[\sum_{k=0}^{n} \varepsilon^{k+1} v_{k}\right] \\
= & L_{1}\left[w_{0}\right]+\left\{\frac{\partial^{2} v_{0}}{\partial \tau^{2}}+a(x, 0) \frac{\partial v_{0}}{\partial \tau}\right\} \\
& +\sum_{k=1}^{n} \varepsilon^{k}\left(L_{1}\left[w_{k}\right]+L_{2}\left[w_{k-1}\right]\right) \\
& +\sum_{k=1}^{n} \varepsilon^{k}\left\{\frac{\partial^{2} v_{k}}{\partial \tau^{2}}+a(x, 0) \frac{\partial v_{k}}{\partial \tau}+\Phi_{k}(x, \tau)\right\} \\
& +R(x, t ; \varepsilon)
\end{aligned}
$$

where the functions $\Phi_{k}(x, \tau)$ and $R(x, t ; \varepsilon)$ are defined by

$$
\begin{aligned}
\Phi_{k}(x, \tau)= & \sum_{j=0}^{k-1} \tilde{c}_{j}(x) \tau^{j} \frac{\partial^{2} v_{k-j}-1}{\partial x \partial \tau}+\sum_{j=0}^{k-2} c_{j}^{*}(x) \tau^{j} \frac{\partial^{2} v_{k-j-2}}{\partial x^{2}} \\
& +\sum_{j=1}^{k} a_{j}(x) \tau^{j} \frac{\partial v_{k-j}}{\partial \tau}+\sum_{j=0}^{k-1} b_{j}(x) \tau^{j} \frac{\partial v_{k-j-1}}{\partial x} \\
& +\sum_{j=0}^{k-1} a_{j}(x) \tau^{j} v_{k-j-1}
\end{aligned}
$$

and

$$
\begin{aligned}
R(x, t ; \varepsilon)= & \varepsilon^{n+1} L_{2}\left[w_{n}\right]+\sum_{k=n}^{2 n} \varepsilon^{k+1}\left\{\sum_{j=k-n}^{n} \tilde{c}_{j}(x) \tau^{j} \frac{\partial^{2} v_{k-j}}{\partial x \partial \tau}\right\} \\
& +\varepsilon^{n+1} \sum_{j=0}^{n-1} c_{j}^{*}(x) \tau^{j} \frac{\partial^{2} v_{n-j-1}}{\partial x^{2}} \\
& +\sum_{k=n}^{2 n} \varepsilon^{k+2}\left\{\sum_{j=k-n}^{n} c_{j}^{*}(x) \tau^{j} \frac{\partial^{2} v_{k-j}}{\partial x^{2}}\right\} \\
& +\tilde{c}_{n+1}(x, t) \varepsilon^{n+1} \tau^{n+1} \sum_{k=0}^{n} \varepsilon^{k+1} \frac{\partial^{2} v_{k}}{\partial x \partial \tau}+
\end{aligned}
$$




$$
\begin{aligned}
& +c_{n+1}^{*}(x, t) \varepsilon^{n+1} \tau^{n+1} \sum_{k=0}^{n} \varepsilon^{k+2} \frac{\partial^{2} v_{k}}{\partial x^{2}} \\
& +\sum_{k=n+1}^{2 n} \varepsilon^{k}\left\{\sum_{j=k-n}^{n} a_{j}(x) \tau^{j} \frac{\partial v_{k-j}}{\partial \tau}\right\} \\
& +a_{n+1}(x, t) \varepsilon^{n+1} \tau^{n+1} \sum_{k=0}^{n} \varepsilon^{k} \frac{\partial v_{k}}{\partial \tau} \\
& +\sum_{k=n}^{2 n} \varepsilon^{k+1}\left\{\sum_{j=k-n}^{n} b_{j}(x) \tau^{j} \frac{\partial v_{k-j}}{\partial x}\right\} \\
& +b_{n+1}(x, t) \varepsilon^{n+1} \tau^{n+1} \sum_{k=0}^{n} \varepsilon^{k+1} \frac{\partial v_{k}}{\partial x} \\
& \left.+\sum_{k=n}^{2 n} \varepsilon^{k+1} \sum_{j=k-n}^{n} d_{j}(x) \tau^{j} v_{k-j}\right\} \\
& +d_{n+1}(x, t) \varepsilon^{n+1} \tau^{n+1} \sum_{k=0}^{n} \varepsilon^{k+1} v_{k} \cdot
\end{aligned}
$$

N.B. Empty sums are to be interpreted as zero.

Putting $t=0$ in (36) we get

$$
\left\{\begin{array}{l}
\tilde{u}(x, 0 ; \varepsilon)=w_{0}(x, 0)+\sum_{k=1}^{n} \varepsilon^{k}\left\{w_{k}(x, 0)+v_{k-1}(x, 0)\right\}+\varepsilon^{n+1} v_{n}(x, 0) \\
\tilde{u}_{t}(x, 0 ; \varepsilon)=\sum_{k=0}^{n} \varepsilon^{k}\left\{\frac{\partial w_{k}}{\partial t}(x, 0)+\frac{\partial v_{k}}{\partial \tau}(x, 0)\right\}
\end{array}\right.
$$

The formal approximation $\tilde{u}$ of the solution $u$ of (34) is now obtained by solving the following set of initial value problems

$$
\begin{aligned}
& \left\{\begin{array}{l}
a(x, t) \frac{\partial w_{0}}{\partial t}+b(x, t) \frac{\partial w_{0}}{\partial x}+d(x, t) w_{0}=f(x, t), \quad(x, t) \in D \\
w_{0}(x, 0)=g(x), \quad-\infty<x<\infty
\end{array}\right. \\
& \left\{\begin{array}{l}
\frac{\partial^{2} v_{0}}{\partial \tau^{2}}+a(x, 0) \frac{\partial v_{0}}{\partial \tau}=0, \quad(x, \tau) \in D \\
\frac{\partial v_{0}}{\partial \tau}(x, 0)=h(x)-\frac{\partial w_{0}}{\partial t}(x, 0), \quad-\infty<x<\infty \\
\lim _{\tau \rightarrow \infty} v_{0}(x, \tau)=0, \quad-\infty<x<\infty
\end{array}\right.
\end{aligned}
$$


and for $k=1,2, \ldots, n$

$$
\begin{aligned}
& \left\{\begin{array}{l}
a(x, t) \frac{\partial w_{k}}{\partial t}+b(x, t) \frac{\partial w_{k}}{\partial x}+d(x, t) w_{k}=-L_{2}\left[w_{k-1}\right], \quad(x, t) \in D \\
w_{k}(x, 0)=-v_{k-1}(x, 0), \quad-\infty<x<\infty
\end{array}\right. \\
& \left\{\begin{array}{l}
\frac{\partial^{2} v_{k}}{\partial \tau^{2}}+a(x, 0) \frac{\partial v_{k}}{\partial \tau}=-\Phi_{k}(x, \tau), \quad(x, \tau) \in D \\
\frac{\partial v_{k}}{\partial \tau}(x, 0)=-\frac{\partial w_{k}}{\partial t}(x, 0), \quad-\infty<x<\infty \\
\lim _{\tau \rightarrow \infty} v_{k}(x, \tau)=0, \quad-\infty<x<\infty .
\end{array}\right.
\end{aligned}
$$

It is easily established that the functions $v_{k}(x, \tau)$ are of the form

$$
v_{k}(x, \tau)=P_{k}(\tau ; x) \exp \{-a(x, 0) \tau\}
$$

with $\mathrm{P}_{\mathrm{k}}(\tau ; \mathrm{x})$ some polynomial in $\tau$ with coefficients depending on $\mathrm{x}$; they are typical so-called boundary layer functions.

The formulae (37) and (38) can now be written as

$$
\left\{\begin{array}{l}
L^{(\varepsilon)}[\tilde{u}]=f(x, t)+R(x, t ; \varepsilon), \quad(x, t) \in D \\
\tilde{u}(x, 0 ; \varepsilon)=g(x)+\varepsilon^{n+1} v_{n}(x, 0), \quad-\infty<x<\infty \\
\tilde{u}_{t}(x, 0 ; \varepsilon)=h(x), \quad-\infty<x<\infty .
\end{array}\right.
$$

One easily verifies that for every compact subset $\Omega \subset \bar{D}$

$$
R(x, t ; \varepsilon)=O\left(\varepsilon^{n+1}\right), \quad \varepsilon \downarrow 0
$$

uniformly valid in $\Omega$. Hence the function $\tilde{u}$ provides a formal approximation (up to order $\varepsilon^{n+1}$ ) for the solution $u$ of problem (34).

REMARK. Note that so far we did not use the timelike condition $\mathrm{ac}_{1}<\mathrm{b}<\mathrm{ac}_{2}$. Apparently a formal approximation can also be constructed if the subcharacteristics are spacelike. It is only in the proof of the correctness of the formal approximation (which follows presently) that the timelike condition becomes essential. 
Introducing the remainder term $z(x, t ; \varepsilon)$ by

(46)

$$
z(x, t ; \varepsilon)=u(x, t ; \varepsilon)-\tilde{u}(x, t ; \varepsilon)
$$

we obtain from (34) and (44)

$$
\left\{\begin{array}{l}
L^{(\varepsilon)}[z]=-R(x, t ; \varepsilon), \quad(x, t) \in D \\
z(x, 0 ; \varepsilon)=-\varepsilon^{n+1} v_{n}(x, 0), \quad-\infty<x<\infty \\
z_{t}(x, 0 ; \varepsilon)=0, \quad-\infty<x<\infty
\end{array}\right.
$$

so using (45) we get from theorem 1

$$
\begin{cases}z(x, t ; \varepsilon)=0\left(\varepsilon^{n+\frac{3}{4}}\right), & \varepsilon \downarrow 0 \\ z_{x}(x, t ; \varepsilon)=0\left(\varepsilon^{n}\right), & \varepsilon+0 \\ z_{t}(x, t ; \varepsilon)=0\left(\varepsilon^{n}\right), & \varepsilon+0\end{cases}
$$

uniformly valid in any compact subset $\Omega \dot{\bar{D}}$.

Hence it follows from (36) and (46) that

$$
\begin{cases}u(x, t ; \varepsilon)=\sum_{k=0}^{n} \varepsilon^{k} w_{k}(x, t)+\sum_{k=0}^{n-1} \varepsilon^{k+1} v_{k}(x, \tau)+0\left(\varepsilon^{n+\frac{3}{4}}\right), & \varepsilon \downarrow 0 \\ u_{x}(x, t ; \varepsilon)=\sum_{k=0}^{n-1} \varepsilon^{k} \frac{\partial w_{k}}{\partial x}(x, t)+\sum_{k=0}^{n-2} \varepsilon^{k+1} \frac{\partial v_{k}}{\partial x}(x, \tau)+0\left(\varepsilon^{n}\right), & \varepsilon \downarrow 0 \\ u_{t}(x, t ; \varepsilon)=\sum_{k=0}^{n-1} \varepsilon^{k} \frac{\partial w_{k}}{\partial t}(x, t)+\sum_{k=0}^{n-1} \varepsilon^{k} \frac{\partial v_{k}}{\partial \tau}(x, \tau)+0\left(\varepsilon^{n}\right), & \varepsilon \downarrow 0\end{cases}
$$

uniformly valid in any compact subset $\Omega \subset \overline{\mathrm{D}}$. Applying these estimates with $\mathrm{n}$ replaced by $\mathrm{n}+1$ we finally obtain

THEOREM 2. Let $u(x, t ; \varepsilon)$ be the solution of the initial value problem $(34)$, where all data are $\mathrm{C}^{\infty}$-functions which in addition satisfy the conditions (35). Then we have for any $\mathrm{n} \in \mathbb{N} \cup\{($ ) $\}$ 


$$
\left\{\begin{array}{l}
u(x, t ; \varepsilon)=\sum_{k=0}^{n} \varepsilon^{k} w_{k}(x, t)+\sum_{k=0}^{n-1} \varepsilon^{k+1} v_{k}(x, \tau)+0\left(\varepsilon^{n+1}\right), \\
u_{x}(x, t ; \varepsilon)=\sum_{k=0}^{n} \varepsilon^{k} \frac{\partial w_{k}}{\partial x}(x, t)+\sum_{k=0}^{n-1} \varepsilon^{k+1} \frac{\partial v_{k}}{\partial x}(x, \tau)+0\left(\varepsilon^{n+1}\right), \varepsilon \downarrow 0 \\
u_{t}(x, t ; \varepsilon)=\sum_{k=0}^{n} \varepsilon^{k} \frac{\partial w_{k}}{\partial t}(x, t)+\sum_{k=0}^{n} \varepsilon^{k} \frac{\partial v_{k}}{\partial \tau}(x, \tau)+0\left(\varepsilon^{n+1}\right), \varepsilon+0
\end{array}\right.
$$

uniformly valid in any compact subset $\Omega \subset \bar{D}$, where $\tau=\frac{t}{\varepsilon}$ and the functions $\mathrm{w}_{\mathrm{k}}$ and $\mathrm{v}_{\mathrm{k}}(\mathrm{k}=0,1, \ldots, \mathrm{n})$ follow successively from $(39),(40),(41)$ and (42).

In case $\mathrm{n}=0$ empty sums are to be interpreted as zero.

COROLLARY 1. It follows from (47) that first approximations $(\mathrm{n}=0)$ for the solution $\mathrm{u}$ of (34) and its first derivatives are given by

$$
\left\{\begin{array}{l}
u(x, t ; \varepsilon)=w_{0}(x, t)+0(\varepsilon), \quad \varepsilon \downarrow 0 \\
u_{x}(x, t ; \varepsilon)=\frac{\partial w_{0}}{\partial x}(x, t)+0(\varepsilon), \quad \varepsilon \downarrow 0 \\
u_{t}(x, t ; \varepsilon)=\frac{\partial w_{0}}{\partial t}(x, t)+\left\{h(x)-\frac{\partial w_{0}}{\partial t}(x, 0)\right\} \exp \left[-a(x, 0) \frac{t}{\varepsilon}\right]+0(\varepsilon), \\
\varepsilon \downarrow 0
\end{array}\right.
$$

uniformly valid in any compact subset $\Omega \subset \overline{\mathrm{D}}$, where $\mathrm{w}_{0}$ is the solution of the reduced problem (39).

COROLLARY 2. It follows from (47) and (43) that for any $\mathrm{n} \in \mathbb{I N} \cup\{0\}$

$$
\begin{cases}u(x, t ; \varepsilon)=\sum_{k=0}^{n} \varepsilon^{k} w_{k}(x, t)+o\left(\varepsilon^{n+1}\right), & \varepsilon+0 \\ u_{x}(x, t ; \varepsilon)=\sum_{k=0}^{n} \varepsilon^{k} \frac{\partial w_{k}}{\partial x}(x, t)+o\left(\varepsilon^{n+1}\right), & \varepsilon+0 \\ u_{t}(x, t ; \varepsilon)=\sum_{k=0}^{n} \varepsilon^{k} \frac{\partial w_{k}}{\partial t}(x, t)+o\left(\varepsilon^{n+1}\right), & \varepsilon+0\end{cases}
$$

uniformly valid in any compact subset $\Omega \subset\{(\mathrm{x}, \mathrm{t}) \mid-\infty<\mathrm{x}<\infty, 0<\delta \leq \mathrm{t}<\infty\}$ where $\delta$ is an arbitrarily small positive constant independent of $\varepsilon$. 
REMARK. If the condition of infinite smoothness of the data is weakened then the expansions (47) are valid for all $n \in \mathbb{I N} \cup\{0\}$ with $n \leq n_{0}$, the bound $n_{0}$ being determined by the differentiability of the data.

\section{Discussion}

The a priori estimate (33a) that has been established in theorem 1 is, under the conditions stated, the sharpest possible in the sense that the factor $\varepsilon^{-\frac{1}{4}}$ in front of the constant $K_{1}$ is the smallest possible. This can easily be verified by considering the initial value problem.

$$
\left\{\begin{array}{l}
\varepsilon\left(\frac{\partial^{2} u}{\partial t^{2}}-\frac{\partial^{2} u}{\partial x^{2}}\right)+\frac{\partial u}{\partial t}=f(x, t ; \varepsilon), \quad(x, t) \in D \\
u(x, 0 ; \varepsilon)=u_{t}(x, 0 ; \varepsilon)=0, \quad-\infty<x<\infty
\end{array}\right.
$$

with

$$
f(x, t ; \varepsilon)=\left\{\varepsilon+t+\left(1-\frac{2 x^{2}}{\varepsilon}\right) t^{2}\right\} \exp \left(-\frac{x^{2}}{\varepsilon}\right) .
$$

Its solution is given by

$$
u(x, t ; \varepsilon)=\frac{1}{2} t^{2} \exp \left(-\frac{x^{2}}{\varepsilon}\right) \text {. }
$$

Defining the compact subset $\Omega$ by

$$
\Omega=\{(x, t) \mid t-2 \leq x \leq 2-t, 0 \leq t \leq 1\}
$$

we have

$$
\left\{\begin{array}{l}
\|f\|_{\Omega}=O\left(\varepsilon^{\frac{1}{4}}\right), \quad \varepsilon \downarrow 0 \\
\sup _{\Omega}|u|=O(1), \quad \varepsilon \downarrow 0
\end{array}\right.
$$

so it follows that the factor $\varepsilon^{-\frac{1}{4}}$ in the right-hand side of (33a) is optimal.

The energy integral method presented in section 2 is an extension of a similar method developed by DE JAGER [26], which will now briefly be 
discussed. DE JAGER considered the initial value problem

$$
\left\{\begin{array}{l}
\varepsilon\left\{\frac{\partial^{2} u}{\partial t^{2}}-c^{2}(x, t) \frac{\partial^{2} u}{\partial x^{2}}\right\}+a(x, t) \frac{\partial u}{\partial t}+b(x, t) \frac{\partial u}{\partial x}=f(x, t), \quad(x, t) \in D \\
u(x, 0 ; \varepsilon)=g(x), \quad-\infty<x<\infty \\
u_{t}(x, 0 ; \varepsilon)=h(x), \quad-\infty<x<\infty
\end{array}\right.
$$

under the conditions

$$
\left\{\begin{array}{l}
a(x, t)>0 \text { in } \bar{D}, \quad c(x, t)>0 \text { in } \bar{D} \\
\frac{|b(x, t)|}{a(x, t)}<c(x, t) \text { in } \bar{D} .
\end{array}\right.
$$

A formal approximation $\tilde{u}$ for the solution $u$ of this problem can be constructed as in section 3. In order to prove the correctness of this approximation one needs an a priori estimate for the remainder term $\mathrm{z}$ which is a solution of the initial value problem

$$
\left\{\begin{array}{l}
\varepsilon\left\{\frac{\partial^{2} z}{\partial t^{2}}-c^{2}(x, t) \frac{\partial^{2} z}{\partial x^{2}}\right\}+a(x, t) \frac{\partial z}{\partial t}+b(x, t) \frac{\partial z}{\partial x}=F(x, t ; \varepsilon), \quad(x, t) \in D \\
z(x, 0 ; \varepsilon)=z_{t}(x, 0 ; \varepsilon)=0, \quad-\infty<x<\infty
\end{array}\right.
$$

with $F(x, t ; \varepsilon)=O(\varepsilon), \varepsilon \downarrow 0$, uniformly valid in any compact subset $\Omega \subset \bar{D}$. Multiplying the differential equation by a special linear combination of $z_{t}$ and $z_{x^{\prime}}$ namely $a z_{t}+b z_{x}$ (which represents the derivative of $z$ along a subcharacteristic) and integrating the resulting formula over a suitably chosen rectangle $\Omega=\left\{(x, t) \mid x_{1} \leq x \leq x_{2}, 0 \leq t \leq T\right\}$ one obtains the pointwise estimate

$$
\sup _{\Omega}|z(x, t ; \varepsilon)| \leq C(\Omega) \varepsilon^{-\frac{1}{2}}\left\|_{F}\right\|_{\Omega}
$$

from which the correctness of the formal approximation follows.

Compared to DE JAGER's method the extended energy integral method used in section 2 yields some obvious improvements. Firstly, by multiplying the differential equation (48) not only by $a z_{t}+b z_{x}$ but also by $z$ we obtain an estimate which is sharper than (49), namely (cf. theorem 1) 


$$
\sup _{\Omega}|z(x, t ; \varepsilon)| \leq C(\Omega) \varepsilon^{-\frac{1}{4}}\left\|_{F}\right\|_{\Omega^{\prime}}
$$

a result which has been shown to be optimal.

Secondly, by integrating over a region with characteristic boundaries instead of over a rectangle it is possible to obtain pointwise estimates for the derivatives $\frac{\partial z}{\partial x}$ and $\frac{\partial z}{\partial t}$, namely (cf. theorem 1)

$$
\sup _{\Omega}\left|\frac{\partial z}{\partial x}\right|+\sup _{\Omega}\left|\frac{\partial z}{\partial t}\right| \leq C(\Omega) \varepsilon^{-1} \sup _{\Omega}|F|
$$

Finally, the extended energy integral method enables us to deal with more general differential equations which compared to (48) contain an additional term $d(x, t) z$ in the left-hand side.

We shall end this section with a discussion of some other work in the same field.

The singularly perturbed initial value problem (1) has also been investigated by BLONDEL [3]. The following general result was established: if the solution $u$ of (1) tends for $\varepsilon \downarrow 0$ pointwise to a continuously differentiable limit function $w$ in a compact subset $\Omega \subset \bar{D}$ which is bounded by the line $t=0$ and two characteristics (in case of timelike subcharacteristics) or by the line $t=0$, a characteristic and a subcharacteristic (in case of spacelike subcharacteristics) (see figure 10 ) then $w \equiv w_{0}$ in $\Omega$, where $\mathrm{w}_{0}$ is the solution of the reduced problem.

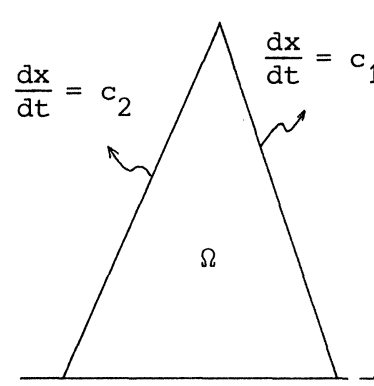

Timelike subcharacteristics

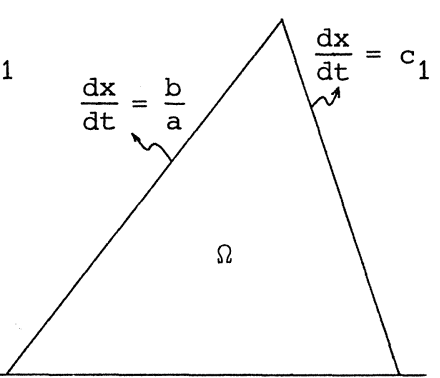

Spacelike

subcharacteristics

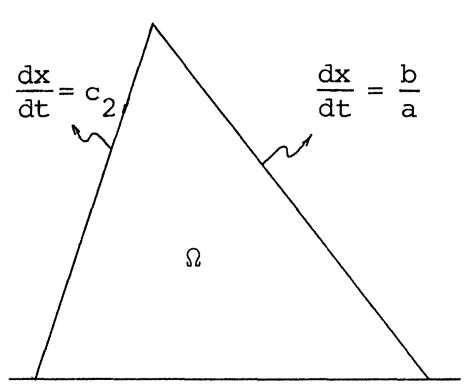

Spacelike

subcharacteristics

Figure 10: The region $\Omega$ 
From this result BLONDEL concluded that the solution $u$ of problem (1) with spacelike subcharacteristics cannot converge pointwise to a $C^{1}$-function in a region $\Omega$ as described above, unless all data are suitably chosen constants. Since example 2 at page 46 contradicts this conclusion a further investigation of problem (1) with spacelike subcharacteristics is justified. For timelike subcharacteristics and a positive coefficient a BLONDEL showed that

$$
u(x, t ; \varepsilon)=w_{0}(x, t)+o\left(\varepsilon^{1 / 8}\right), \quad \varepsilon+0
$$

uniformly valid in any compact subset $\Omega \subset \overline{\mathrm{D}}$, where $\mathrm{w}_{0}$ denotes the solution of the reduced problem. Note that this result is considerably weaker than the result mentioned in corollary 1 at page 70 .

It was also shown in [3] that the derivatives $\frac{\partial u}{\partial x}$ and $\frac{\partial u}{\partial t}$ converge in $L_{2}$-norm to the corresponding derivatives $\frac{\partial \mathrm{w}_{0}}{\partial \mathrm{x}}$ and $\frac{\partial \mathrm{w}_{0}}{\partial t}$ of the solution of the reduced problem as $\varepsilon$ tends to zero. However, pointwise convergence of these derivatives remained an open question. This question seems to have been answered for the first time by SMITH \& PALMER [46] and SMITH [44] for hyperbolic systems with constant coefficients (see further on). Our results (47) for hyperbolic equations with variable coefficients correspond with those obtained by SMITH and PALMER.

BLONDEL [3], [4] investigated also the behaviour of the solution $u$ of (1) with timelike subcharacteristics and a negative coefficient a. It was shown that if the functions $f, g$ and $h$ in (1) are given by $f(x, t ; \varepsilon) \equiv F(x)$, $g(x ; \varepsilon) \equiv h(x ; \varepsilon) \equiv 0$ with $F$ a smooth positive-valued function independent of $t$ and $\varepsilon$, then the solution $u$ of (1) tends exponentially to infinity in the interior of $\bar{D}$ as $\varepsilon$ approaches zero. A consequence of this result is the following: suppose that there exists for suitably chosen functions $f, g$ and $\mathrm{h}$ a solution $\mathrm{u}$ of (1) which converges pointwise to a limit function as $\varepsilon$ tends to zero. Then it suffices to replace $f$ by $f+F$ (with $F$ independent of $t$ and $\varepsilon$, positive and arbitrarily small) in order that the solution of the resulting initial value problem tends exponentially to infinity as $\varepsilon$ approaches zero. In other words, the convergence of $u$ is a phenomenon which is unstable with respect to the right-hand side function $f$.

We strongly believe that the solution $u$ of problem (1) with a negative coefficient a tends exponentially to infinity in every point $(x, t)$ with 
$t \geq \delta>0$ as $\varepsilon$ tends to zero, regardless whether the subcharacteristics are spacelike or timelike (cf. chapter I, section 3); an exception has to be made for very special choices of the functions $f, g$ and $h$ (for instance $\mathrm{f} \equiv \mathrm{g} \equiv \mathrm{h} \equiv 0$ ). However, so far we have not been able to prove this conjecture.

Singularly perturbed initial value problems in more than one space variable have been studied by BLONDEL [4] and DZAVADOV [7]. It was shown by these authors that the solution $u$ and its first derivatives converge in $\mathrm{L}_{2}$-norm to the solution $\mathrm{w}_{0}$ of the reduced problem and the corresponding derivatives of $w_{0}$ as $\varepsilon$ tends to zero.

SMITH [44] studied the singularly perturbed hyperbolic system

$$
\begin{cases}\varepsilon\left(\frac{\partial u}{\partial t}+\frac{\partial u}{\partial x}\right)+A_{1} u+B_{1} v=f_{1}(x, t), & (x, t) \in D \\ \varepsilon\left(\frac{\partial v}{\partial t}-\frac{\partial v}{\partial x}\right)+A_{2} u+B_{2} v=f_{2}(x, t), & (x, t) \in D\end{cases}
$$

with $u(x, 0 ; \varepsilon)$ and $v(x, 0 ; \varepsilon)$ prescribed for $-\infty<x<\infty$.

It was shown that if the constant coefficients $A_{1}, B_{1}, A_{2}$ and $B_{2}$ satisfy the conditions

$$
A_{1} \geq 0, \quad B_{2} \geq 0, \quad A_{1}+B_{2}>0, \quad 0 \leq A_{2} B_{1} \leq A_{1} B_{2}
$$

then the solution $u, v$ of (50) is for small values of $\varepsilon$ approximately given by a solution of the reduced problem obtained from $(50)$ by putting $\varepsilon=0$.

A simplified version of (50) with $A_{1}=B_{1}=A_{2}=B_{2}=\frac{1}{2}$ and $f_{1}=f_{2}=f$ was investigated by SMITH \& PALMER [46].

There is an overlap between our results and those of SMITH. One easily verifies that with the special choice

$$
\left\{\begin{array}{l}
A_{1}=A_{2}=\frac{1}{2}(a-b), \quad B_{1}=B_{2}=\frac{1}{2}(a+b) \\
f_{1}(x, t)=f_{2}(x, t)=f(x, t) \\
u=\frac{\partial \phi}{\partial t}-\frac{\partial \phi}{\partial x}, \quad v=\frac{\partial \phi}{\partial t}+\frac{\partial \phi}{\partial x}
\end{array}\right.
$$


(50) is equivalent to the second order equation

$$
\varepsilon\left(\frac{\partial^{2} \phi}{\partial t^{2}}-\frac{\partial^{2} \phi}{\partial x^{2}}\right)+a \frac{\partial \phi}{\partial t}+b \frac{\partial \phi}{\partial x}=f(x, t), \quad(x, t) \in D
$$

with $\phi(x, 0 ; \varepsilon)$ and $\phi_{t}(x, 0 ; \varepsilon)$ prescribed for $-\infty<x<\infty$.

In this case the conditions (51) take the form

$$
a>0, \quad|b| \leq a
$$

so for constant coefficients our results correspond with those of SMITH.

Results obtained by means of a comparison technique have recently been published by WEINSTEIN \& SMITH [55].

The a priori estimates obtained in theorem 1 will play an important role in the investigations of nonlinear initial value problems. We shall deal with these problems in chapter IV.

Moreover, the energy integral method underlying the proof of theorem 1 can be modified to yield a priori estinates for solutions of singularly perturbed initial-boundary value problems. The chapters V and VI will be devoted to this subject. 
CHAPTER IV

INITIAL VALUE PROBLEMS FOR NONLINEAR

HYPERBOLIC DIFFERENTIAL EQUATIONS

In this chapter we shall extend the linear theory developed in chapter III to nonlinear initial value problems. In particular we shall investigate the quasilinear problems

$$
\left\{\begin{array}{l}
\varepsilon L_{2}[u]+M_{1}^{(\varepsilon)}[u]=f(x, t ; \varepsilon), \quad(x, t) \in D \\
u(x, 0 ; \varepsilon)=u_{t}(x, 0 ; \varepsilon)=0, \quad-\infty<x<\infty
\end{array}\right.
$$

and

$$
\begin{cases}\varepsilon L_{2}[u]+M_{1}[u]=0, & (x, t) \in D \\ u(x, 0 ; \varepsilon)=f(x), & -\infty<x<\infty \\ u_{t}(x, 0 ; \varepsilon)=g(x), & -\infty<x<\infty\end{cases}
$$

where $\varepsilon$ is a small positive parameter, i.e. $0<\varepsilon<<1$, and the region $\mathrm{D}$ is the upper half-plane

$$
D=\{(x, t) \mid-\infty<x<\infty, 0<t<\infty\} .
$$

Here the hyperbolic operator $\mathrm{I}_{2}$ is defined by

$$
L_{2}[u]=\frac{\partial^{2} u}{\partial t^{2}}-c^{2}(x, t) \frac{\partial^{2} u}{\partial x^{2}}
$$

with $c(x, t)>0$ in $\bar{D}$, and the quasilinear operators $M_{1}^{(\varepsilon)}$ and $M_{1}$ are given by 
(4a)

$$
\left\{\begin{array}{l}
M_{1}^{(\varepsilon)}[u]=a(x, t, u ; \varepsilon) \frac{\partial u}{\partial t}+b(x, t, u ; \varepsilon) \frac{\partial u}{\partial x}+d(x, t, u ; \varepsilon) \\
M_{1}[u]=a(x, t, u) \frac{\partial u}{\partial t}+b(x, t, u) \frac{\partial u}{\partial x}+d(x, t, u) .
\end{array}\right.
$$

Under appropriate conditions on the data we shall establish in section 1 the existence of a solution for problem (1); among other things it will be required that

$$
\varepsilon L_{2}[0]+M_{1}^{(\varepsilon)}[0]=0
$$

and also that the right-hand side function $f(x, t ; \varepsilon)$ is of sufficiently small order in $\varepsilon$. The existence proof is similar to the proof that we gave in section 1 of chapter II for ordinary differential equations: using a priori estimates for the solution of the linearized problem (which can be obtained from theorem 1 in chapter III) we can apply theorem 1 of chapter II which leads to the existence of a solution for problem (1), together with an estimate for this solution.

In section 2 we shall construct a formal approximation $\tilde{u}$ for the solution $u$ of (2). The correctness of this approximation will then be verified by applying the results of section 1 to the remainder term.

Section 3 will be devoted to a further discussion of the construction of the formal approximation.

In section 4 we shall deal with two special cases of problem (2) in which the coefficients $a$ and $b$ are independent of $u$.

REMARK. It is possible to consider the problems (1) and (2) with a more general hyperbolic operator $\mathrm{L}_{2}$ defined by

$$
L_{2}[u]=\frac{\partial^{2} u}{\partial t^{2}}+\left\{c_{1}(x, t)+c_{2}(x, t)\right\} \frac{\partial^{2} u}{\partial x \partial t}+c_{1}(x, t) c_{2}(x, t) \frac{\partial^{2} u}{\partial x^{2}}
$$

where $c_{1}(x, t)<c_{2}(x, t)$ in $\bar{D}$ (cf. chapter III). Although we restrict ourselves for reasons of simplicity to the case where $\mathrm{L}_{2}$ represents the wave operator, that is

$$
-c_{1}(x, t) \equiv c_{2}(x, t) \equiv c(x, t)>0 \quad \text { in } \bar{D},
$$

it is emphasized that more general problems with $\mathrm{L}_{2}$ given by (6) can be 
treated in a similar way.

\section{Existence and a priori estimates}

We consider the initial value problem (1) where the operators $\mathrm{L}_{2}$ and $M_{1}^{(\varepsilon)}$ are defined by (3) and (4a). In order to comply with the condition (5) (cf. theorem 1 of chapter II) we assume that

$$
d(x, t, 0 ; \varepsilon) \equiv 0 \text { for }(x, t) \in \bar{D} \text { and } 0<\varepsilon<1
$$

This assumption can be made without loss of generality. Apart from (7) we impose the following conditions on the data:

(8)

$$
\left\{\begin{array}{l}
c(x, t) \in C^{1}(\bar{D}) \\
c(x, t)>0 \text { in } \bar{D} \\
c(x, t) \text { is uniformly bounded in any strip } \\
\{(x, t) \mid-\infty<x<\infty, \quad 0 \leq t \leq T\}
\end{array}\right.
$$

for each compact subset $\Omega \subset \bar{D}$ there exists a positive constant $\varepsilon_{1}$ (depending on $\Omega$ ) such that

(i) $\quad a(x, t, 0 ; \varepsilon) \in C^{1}(\Omega), \quad b(x, t, 0 ; \varepsilon) \in c^{1}(\Omega)$

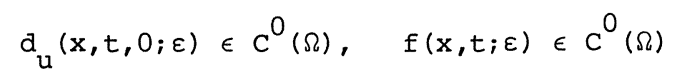

valid for every value of $\varepsilon$ satisfying $0<\varepsilon \leq \varepsilon_{1}$

(ii) the functions $a(x, t, 0 ; \varepsilon), b(x, t, 0 ; \varepsilon)$ and their first derivatives as well as the function $d_{u}(x, t, 0 ; \varepsilon)$ are uniformly bounded in $\Omega$ for $0<\varepsilon \leq \varepsilon_{1}$

(iii) $\quad a(x, t, 0 ; \varepsilon) \geq a_{0}>0$

valid for all $(x, t) \in \Omega$ and all $\varepsilon \in\left(0, \varepsilon_{1}\right]$, where $a_{0}$ is some constant depending on $\Omega$ and $\varepsilon_{1}$ but independent of $\varepsilon$ 
(9)

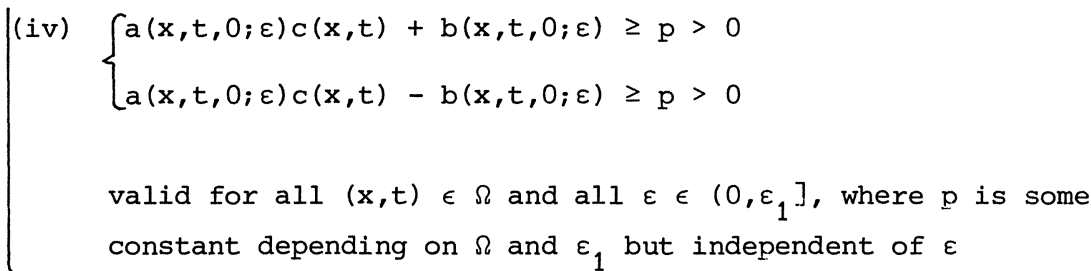

for each compact subset $\Omega \subset \bar{D}$ there exist a positive constant $U_{0}$ and a positive constant $\varepsilon_{2}$ (depending on $\Omega$ and $U_{0}$ ) such that

(i) $\quad a(x, t, u ; \varepsilon) \in c^{0}(\Omega), \quad b(x, t, u ; \varepsilon) \in c^{0}(\Omega), \quad d(x, t, u ; \varepsilon) \in c^{0}(\Omega)$

valid for all $u \in\left[-\mathrm{U}_{0}, \mathrm{U}_{0}\right]$ and all $\varepsilon \in\left(0, \varepsilon_{2}\right]$

(ii) the derivatives $a_{u}(x, t, u ; \varepsilon)$ and $b_{u}(x, t, u ; \varepsilon)$ exist and are uniformly bounded in $\Omega \times\left[-\mathrm{U}_{0}, \mathrm{U}_{0}\right]$ for $0<\varepsilon \leq \varepsilon_{2}$

(10)

(iii) the derivative $d_{u}(x, t, u ; \varepsilon)$ exists in $\Omega \times\left[-U_{0}, U_{0}\right]$ for $0<\varepsilon \leq \varepsilon_{2}$ and satisfies a Lipschitz condition with exponent $\alpha>0$, viz.

$\left|d_{u}(x, t, u ; \varepsilon)-d_{u}(x, t, 0 ; \varepsilon)\right| \leq c\left(\Omega, u_{0}, \varepsilon_{2}\right)|u|^{\alpha}$

valid for all $(\mathrm{x}, \mathrm{t}, \mathrm{u}) \in \Omega \times\left[-\mathrm{U}_{0}, \mathrm{u}_{0}\right]$ and all $\varepsilon \in\left(0, \varepsilon_{2}\right]$. Here $\mathrm{C}\left(\Omega, \mathrm{U}_{0}, \varepsilon_{2}\right)$ is some positive constant depending on $\Omega, \mathrm{U}_{0}$ and $\varepsilon_{2}$ but independent of $x, t$, $u$ or $\varepsilon$.

In order to establish the existence of a solution for problem (1) we consider a compact subset $\Omega \subset \bar{D}$ which is bounded by the lines $t=0$ and $t=T$ $(T>0)$ and by two characteristics of the operator $\mathrm{L}_{2}$, one with a negative slope, the other with a positive slope (see figure 1). 


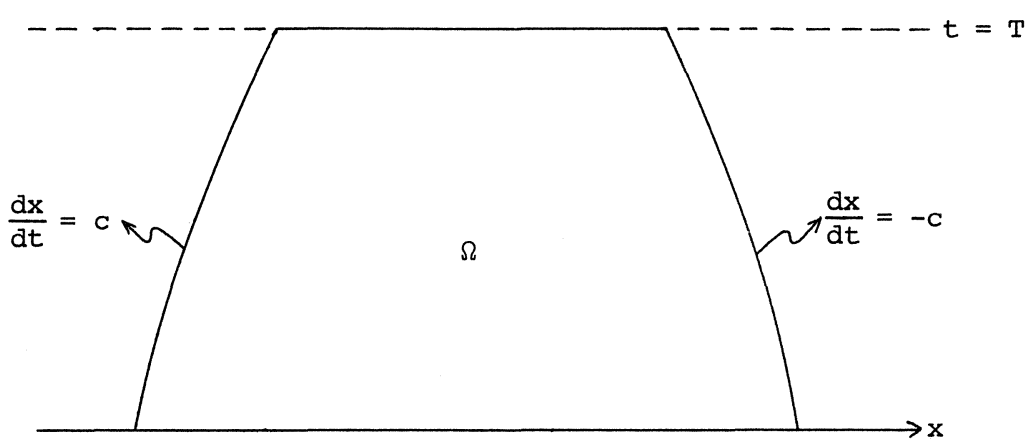

Figure 1

The existence proof will be based on theorem 1 of chapter II. In terms of this theorem we introduce for any fixed value of $\varepsilon$ with $0<\varepsilon \leq \varepsilon_{0}=\min \left(\varepsilon_{1}, \varepsilon_{2}\right)$ the Banach space

$$
B=\left\{u \mid u \in C^{0}(\Omega)\right\}
$$

with norm

$$
|u|_{0}=\sup _{\Omega}|u|
$$

and the normed linear space

$$
R=\left\{u \mid u \in C^{1}(\Omega), L_{2}[u] \in C^{0}(\Omega), u(x, 0)=u_{t}(x, 0)=0\right\}
$$

with norm

$$
|u|_{1}^{(\varepsilon)}=\sup _{\Omega}|u|+\varepsilon^{\frac{3}{4}} \sup _{\Omega}\left|\frac{\partial u}{\partial x}\right|+\varepsilon^{\frac{3}{4}} \sup _{\Omega}\left|\frac{\partial u}{\partial t}\right| .
$$

We state here explicitly that the expression $\mathrm{L}_{2}[\mathrm{u}]$ in (11) may contain weak second derivatives.

Let $B(\rho)$ be a ball in $R$ with radius $\rho$, that is

$$
B(\rho)=\left\{\left.u \in R|| u\right|_{1} ^{(\varepsilon)} \leq \rho\right\} .
$$


Choosing $\bar{\rho}=U_{0}$ we define the operators $F: B(\bar{\rho}) \rightarrow B, L: R \rightarrow B$ and $\Psi: B(\bar{\rho}) \rightarrow B$ by

$$
\left\{\begin{aligned}
& F[u]= \varepsilon L_{2}[u]+M_{1}^{(\varepsilon)}[u] \\
& L[u]= \varepsilon L_{2}[u]+a(x, t, 0 ; \varepsilon) \frac{\partial u}{\partial t}+b(x, t, 0 ; \varepsilon) \frac{\partial u}{\partial x}+d_{u}(x, t, 0 ; \varepsilon) u \\
& \Psi[u]=\{a(x, t, u ; \varepsilon)-a(x, t, 0 ; \varepsilon)\} \frac{\partial u}{\partial t}+\{b(x, t, u ; \varepsilon)-b(x, t, 0 ; \varepsilon)\} \frac{\partial u}{\partial x} \\
&+d(x, t, u ; \varepsilon)-d_{u}(x, t, 0 ; \varepsilon) u .
\end{aligned}\right.
$$

Using the conditions (8) and (9) we obtain from theorem 1 in chapter III

$$
\left|\mathrm{L}^{-1}[\mathrm{v}]\right|_{1}^{(\varepsilon)} \leq \mathrm{M}(\Omega) \varepsilon^{-\frac{1}{4}}|\mathrm{v}|_{0}
$$

valid for all $\varepsilon \in\left(0, \varepsilon_{0}\right]$ and all $\mathrm{v} \in \mathrm{B}$, where $M(\Omega)$ is some positive constant depending on $\Omega$ but independent of $\varepsilon$, and $\varepsilon_{0}$ is some generic constant. So in terms of theorem 1 in chapter II we have

$$
\ell=\frac{\varepsilon^{\frac{1}{4}}}{M(\Omega)}
$$

It can easily be verified that the operator $\Psi$ satisfies a Lipschitz condition. Using the conditions $(10)$ we have for every $\rho \in[0, \bar{\rho}]$ and every $\mathrm{u}, \mathrm{v} \in \mathrm{R}$ with $|\mathrm{u}|_{1}^{(\varepsilon)} \leq \rho,|\mathrm{v}|_{1}^{(\varepsilon)} \leq \rho$ the following relation

$$
\begin{aligned}
& \Psi[u]-\psi[v]= \\
& \{a(x, t, u ; \varepsilon)-a(x, t, 0 ; \varepsilon)\}\left(u_{t}-v_{t}\right)+v_{t}\{a(x, t, u ; \varepsilon)-a(x, t, v ; \varepsilon)\} \\
& \quad+\{b(x, t, u ; \varepsilon)-b(x, t, 0 ; \varepsilon)\}\left(u_{x}-v_{x}\right)+v_{x}\{b(x, t, u ; \varepsilon)-b(x, t, v ; \varepsilon)\} \\
& \quad+d(x, t, u ; \varepsilon)-d(x, t, v ; \varepsilon)-d_{u}(x, t, 0 ; \varepsilon)(u-v)= \\
& u_{u}\left(x, t, \theta_{1} u ; \varepsilon\right)\left(u_{t}-v_{t}\right)+v_{t} a_{u}\left(x, t, v+\theta_{2}(u-v) ; \varepsilon\right)(u-v) \\
& \quad+u b_{u}\left(x, t, \theta_{3} u ; \varepsilon\right)\left(u_{x}-v_{x}\right)+v_{x} b_{u}\left(x, t, v+\Theta_{4}(u-v) ; \varepsilon\right)(u-v) \\
& \quad+\left\{d_{u}\left(x, t, v+\Theta_{5}(u-v) ; \varepsilon\right)-d_{u}(x, t, 0 ; \varepsilon)\right\}(u-v)
\end{aligned}
$$


with $0<\theta_{i}<1(i=1,2, \ldots, 5)$. Hence we obtain, again using (10)

$$
\begin{aligned}
|\Psi[\mathrm{u}]-\Psi[\mathrm{v}]|_{0} \leq & \mathrm{A}(\Omega) \varepsilon^{-\frac{3}{4}} \rho\left\{\varepsilon^{\frac{3}{4}} \sup _{\Omega}\left|\mathrm{u}_{\mathrm{x}}-\mathrm{v}_{\mathrm{x}}\right|+\varepsilon_{\Omega}^{\frac{3}{4}} \sup _{\Omega}\left|\mathrm{u}_{t}-\mathrm{v}_{t}\right|\right\} \\
& +\mathrm{B}(\Omega) \max \left(\varepsilon^{-\frac{3}{4}} \rho, \rho^{\alpha}\right) \sup _{\Omega}|\mathrm{u}-\mathrm{v}| \\
\leq & \mathrm{C}(\Omega) \max \left(\varepsilon^{-\frac{3}{4}} \rho, \rho^{\alpha}\right)|\mathrm{u}-\mathrm{v}|_{1}^{(\varepsilon)}
\end{aligned}
$$

valid for $0<\varepsilon \leq \varepsilon_{0}$. Here $A(\Omega), B(\Omega)$ and $C(\Omega)$ are positive constants which depend (apart from $U_{0}$ and $\varepsilon_{0}$ ) on the region $\Omega$. They do not, however, depend on $\varepsilon$.

Using the terminology of theorem 1 in chapter II we conclude from the foregoing that

$$
m(\rho)=C(\Omega) \max \left(\varepsilon^{-\frac{3}{4}} \rho, \rho^{\alpha}\right)
$$

N.B. The following simplifications are easily established: if $\mathrm{a}$ and $\mathrm{b}$ do not depend on $u$ then $\mathrm{m}(\rho)=\mathrm{c}(\Omega) \rho^{\alpha}$; if $\mathrm{d}$ is linear in $\mathrm{u}$ then $\mathrm{m}(\rho)=\mathrm{C}(\Omega) \varepsilon^{-\frac{3}{4}} \rho$.

Solving $m\left(\rho_{0}\right)=\frac{1}{2} l$ we obtain

$$
\rho_{0}=N(\Omega) \varepsilon^{\lambda}
$$

with

$$
\lambda= \begin{cases}1 & \text { if } \mathrm{d} \text { is linear in } \mathrm{u} \\ \frac{1}{4 \alpha} & \text { if } \mathrm{a} \text { and } \mathrm{b} \text { do not depend on } \mathrm{u} \\ \max \left(1, \frac{1}{4 \alpha}\right) & \text { otherwise }\end{cases}
$$

and $N(\Omega)$ some positive constant depending on $\Omega$ but independent of $\varepsilon$. Finally, applying theorem 1 of chapter II we obtain

THEOREM 1. Let $\Omega$ be some compact subset of $\overline{\mathrm{D}}$ as shown in figure 1 . Then there exist positive constants $\varepsilon_{0}, \mathrm{M}(\Omega)$ and $\mathrm{N}(\Omega)$ (depending on $\Omega$ but independent of $\varepsilon$ ) such that for $(\mathrm{x}, \mathrm{t}) \in \Omega$ and $0<\varepsilon \leq \varepsilon_{0}$ problem (1) under 
the conditions (7), (8), (9) and (10) has a solution $u \in R$, provided that $f$ is sufficiently small, viz.

$$
\sup _{\Omega}|f| \leq \frac{N(\Omega)}{2 M(\Omega)} \varepsilon^{\lambda+\frac{1}{4}}
$$

Here the normed linear space $\mathrm{R}$ is given by (11) and (12), and the positive constant $\lambda$ is given by (13).

The solution u satisfies the estimate

$$
|\mathrm{u}|_{1}^{(\varepsilon)} \leq 2 \mathrm{M}(\Omega) \varepsilon^{-\frac{1}{4}} \sup _{\Omega}|\mathrm{f}|
$$

REMARKS.

1. Since theorem 1 of chapter II applies to nonlinear operators F mapping a (ball belonging to a) normed linear space into a Banach space it was sufficient to verify that the linear space $R$ defined by (11) and (12) is a normed linear space; we did not have to prove the linear space $R$ (supplied with some suitable norm) to be a Banach space. Such a proof would have been necessary if we had applied the theory developed by VAN HARTEN [23, chapter 7] for nonlinear operators F mapping a (linear subspace of a) Banach space into a normed linear space.

2. Note that in theorem 1 the functions $a(x, t, u ; \varepsilon), b(x, t, u ; \varepsilon)$ and $d(x, t, u ; \varepsilon)$ are allowed to have singularities in $u$ (cf. chapter II).

3. A similar theorem can be formulated for more general nonlinear initial value problems of the type

$$
\left\{\begin{array}{l}
\varepsilon L_{2}[u]+F(x, t, \vec{u} ; \varepsilon)=f(x, t ; \varepsilon), \quad(x, t) \in D \\
u(x, 0 ; \varepsilon)=u_{t}(x, 0 ; \varepsilon)=0, \quad-\infty<x<\infty
\end{array}\right.
$$

(where $\vec{u}$ denotes the vector $\left(u_{,} u_{t}, u_{x}\right)$ ) provided that suitable conditions are imposed on the nonlinear function $F$, namely

(i) $\quad F(x, t, \overrightarrow{0} ; \varepsilon) \equiv 0$ for $(x, t) \in \bar{D}$ and $0<\varepsilon<1$,

(ii) in order that theorem 1 of chapter III is applicable to the linearized problem one must require that (9) holds, with $a(x, t, 0 ; \varepsilon), b(x, t, 0 ; \varepsilon)$ and $d_{u}(x, t, 0 ; \varepsilon)$ replaced by $F_{u_{t}}(x, t, \overrightarrow{0} ; \varepsilon)$, 


$$
F_{u_{x}}(x, t, \overrightarrow{0} ; \varepsilon) \text { resp. } F_{u}(x, t, \overrightarrow{0} ; \varepsilon)
$$

(iii) in order that the nonlinear part $\Psi$ given by

$$
\begin{array}{r}
\Psi(x, t, \vec{u} ; \varepsilon)=F(x, t, \vec{u} ; \varepsilon)-F_{u_{t}}(x, t, \overrightarrow{0} ; \varepsilon) \frac{\partial u}{\partial t}-F_{u_{x}}(x, t, \overrightarrow{0} ; \varepsilon) \frac{\partial u}{\partial x} \\
-F_{u}(x, t, \overrightarrow{0} ; \varepsilon) u
\end{array}
$$

satisfies a Lipschitz condition it is sufficient to require that there exist for every compact subset $\Omega \subset \bar{D}$ positive constants $U_{0}$ and $\varepsilon_{0}$ (depending on $\Omega$ ) such that the function $F$ and its second derivatives with respect to $u_{,} u_{t}$ and $u_{x}$ are continuous and uniformly bounded for $(x, t) \in \Omega,|u| \leq U_{0},\left|u_{t}\right| \leq U_{0},\left|u_{x}\right| \leq U_{0}$ and $0<\varepsilon \leq \varepsilon_{0}$.

\section{Asymptotic expansions}

As an application of the a priori estimates obtained in the foregoing section we shall construct an asymptotic expansion into powers of $\varepsilon$ for the solution $u$ of the quasilinear initial value problem

$$
\left\{\begin{array}{l}
L^{(\varepsilon)}[u] \equiv \varepsilon L_{2}[u]+M_{1}[u]=0, \quad(x, t) \in D \\
u(x, 0 ; \varepsilon)=f(x), \\
u_{t}(x, 0 ; \varepsilon)=g(x), \quad-\infty<x<\infty
\end{array}\right.
$$

where the operators $L_{2}$ and $M_{1}$ are defined by (3) and (4b). For simplicity it will be assumed that all data are $c^{\infty}$-functions, that. is

$$
\left\{\begin{array}{l}
a(x, t, u) \in C^{\infty}(\bar{D} \times I R), \quad b(x, t, u) \in C^{\infty}(\bar{D} \times I R), \\
c(x, t) \in C^{\infty}(\bar{D}), \quad d(x, t, u) \in C^{\infty}(\bar{D} \times I R), \\
f(x) \in C^{\infty}(I R), \quad g(x) \in C^{\infty}(I R) .
\end{array}\right.
$$

In addition we require that 
(17)

$$
\left\{\begin{array}{l}
c(x, t)>0 \quad \text { in } \bar{D} \\
c(x, t) \text { is uniformly bounded in any strip } \\
\{(x, t) \mid-\infty<x<\infty, 0 \leq t \leq T\} \\
a(x, t, u)>0 \quad \text { in } \bar{D} \times \mathbb{R} \\
|b(x, t, u)|<a(x, t, u) c(x, t) \quad \text { in } \bar{D} \times \mathbb{I R} .
\end{array}\right.
$$

A formal approximation $\tilde{u}$ for the solution $u$ of problem (15) can be constructed as follows: we put

$$
\tilde{u}(x, t ; \varepsilon)=w_{0}(x, t)+\varepsilon v_{0}(x, \tau)+\varepsilon w_{1}(x, t)+\varepsilon^{2} v_{1}(x, \tau)
$$

where $\tau=\frac{t}{\varepsilon}$ and $w_{k}$ and $v_{k}(k=0,1)$ are functions which have to be determined. For the moment we shall assume that the functions $w_{k}$ and $v_{k}(k=0,1)$ have the following properties

$$
\left\{\begin{array}{l}
w_{k}(x, t) \in C^{\infty}(\bar{D}), \quad v_{k}(x, \tau) \in C^{\infty}(\bar{D}) \\
\text { for any } n, m \in \mathbb{I N} u\{0\} \text { and any polynomial } P(\tau ; x) \text { in } \tau \text { with } \\
\text { coefficients depending continuously on } x \text { the expression } \\
P(\tau ; x) \frac{\partial^{n+m} v_{k}}{\partial \tau^{n} \partial x^{m}} \\
\text { is uniformly bounded in every semi-infinite strip } \\
x_{1} \leq x \leq x_{2}, 0 \leq \tau<\infty .
\end{array}\right.
$$

We shall verify the validity of these properties later on, when the functions $w_{k}$ and $v_{k}$ have been determined explicitly.

Expanding the coefficient $a(x, t, \tilde{u})$ we obtain

$$
\begin{aligned}
a(x, t, \tilde{u}) & =a\left(x, t, w_{0}+\varepsilon v_{0}+\varepsilon w_{1}+\varepsilon^{2} v_{1}\right) \\
& =a\left(x, t, w_{0}\right)+\varepsilon\left(v_{0}+w_{1}\right) \frac{\partial a}{\partial u}\left(x, t, w_{0}\right)+o\left(\varepsilon^{2}\right)
\end{aligned}
$$


uniformly valid in any compact subset $\Omega \subset \bar{D}$.

Using

$$
\frac{\partial \tilde{u}}{\partial t}=\frac{\partial w_{0}}{\partial t}+\frac{\partial v_{0}}{\partial \tau}+\varepsilon \frac{\partial w_{1}}{\partial t}+\varepsilon \frac{\partial v_{1}}{\partial \tau}
$$

we obtain

$$
\begin{aligned}
a(x, t, \tilde{u}) \frac{\partial \tilde{u}}{\partial t}= & a\left(x, t, w_{0}\right) \frac{\partial w_{0}}{\partial t}+a\left(x, t, w_{0}\right) \frac{\partial v_{0}}{\partial \tau} \\
& +\varepsilon\left[a\left(x, t, w_{0}\right) \frac{\partial w_{1}}{\partial t}+a\left(x, t, w_{0}\right) \frac{\partial v_{1}}{\partial \tau}\right. \\
& \left.+\left(w_{1}+v_{0}\right)\left(\frac{\partial w_{0}}{\partial t}+\frac{\partial v_{0}}{\partial \tau}\right) \frac{\partial a}{\partial u}\left(x, t, w_{0}\right)\right] \\
& +o\left(\varepsilon^{2}\right)
\end{aligned}
$$

uniformly valid in any compact subset $\Omega \subset \bar{D}$.

So putting $t=\varepsilon \tau$ and expanding into powers of $\varepsilon$ we arrive at

$$
\begin{aligned}
& a(x, t, \tilde{u}) \frac{\partial \tilde{u}}{\partial t}= \\
& a\left(x, t, w_{0}\right) \frac{\partial w_{0}}{\partial t}+a\left(x, 0, w_{0}(x, 0)\right) \frac{\partial v_{0}}{\partial \tau} \\
& +\varepsilon\left[\left\{\frac{\partial a}{\partial t}\left(x, 0, w_{0}(x, 0)\right)+\frac{\partial a}{\partial u}\left(x, 0, w_{0}(x, 0)\right) \frac{\partial w_{0}}{\partial t}(x, 0)\right\} \tau \frac{\partial v_{0}}{\partial \tau}\right. \\
& +a\left(x, t, w_{0}\right) \frac{\partial w_{1}}{\partial t}+a\left(x, 0, w_{0}(x, 0)\right) \frac{\partial v_{1}}{\partial \tau} \\
& +\frac{\partial a}{\partial u}\left(x, t, w_{0}\right) w_{1} \frac{\partial w_{0}}{\partial t}+\frac{\partial a}{\partial u}\left(x, 0, w_{0}(x, 0)\right) w_{1}(x, 0) \frac{\partial v_{0}}{\partial \tau} \\
& \left.+\frac{\partial a}{\partial u}\left(x, 0, w_{0}(x, 0)\right) \frac{\partial w_{0}}{\partial t}(x, 0) v_{0}+\frac{\partial a}{\partial u}\left(x, 0, w_{0}(x, 0)\right) v_{0} \frac{\partial v_{0}}{\partial \tau}\right] \\
& +0\left(\varepsilon^{2}\right)
\end{aligned}
$$

uniformly valid in each compact subset. $\Omega \subset \overline{\mathrm{D}}$. 
$N . B$. Unless otherwise stated the arguments are $x, t$ or $x, \tau$. So $w_{0}$ stands for $w_{0}(x, t), v_{0}$ stands for $v_{0}(x, \tau)$ etc.

A similar expansion of the other terms in $L^{(\varepsilon)}[\tilde{u}]$ leads to the following expansion of the operator:

$$
\begin{aligned}
L^{(\varepsilon)}[\tilde{u}]= & a\left(x, t, w_{0}\right) \frac{\partial w_{0}}{\partial t}+b\left(x, t, w_{0}\right) \frac{\partial w_{0}}{\partial x}+d\left(x, t, w_{0}\right) \\
& +\frac{\partial^{2} v_{0}}{\partial \tau^{2}}+a\left(x, 0, w_{0}(x, 0)\right) \frac{\partial v_{0}}{\partial \tau} \\
& +\varepsilon\left[a\left(x, t, w_{0}\right) \frac{\partial w_{1}}{\partial t}+b\left(x, t, w_{0}\right) \frac{\partial w_{1}}{\partial x}+\tilde{d}\left(x, t, w_{0}\right) w_{1}+\Phi_{1}(x, t)\right] \\
& +\varepsilon\left[\frac{\partial^{2} v_{1}}{\partial \tau^{2}}+a\left(x, 0, w_{0}(x, 0)\right) \frac{\partial v_{1}}{\partial \tau}+\Psi_{1}(x, \tau)\right]+0\left(\varepsilon^{2}\right)
\end{aligned}
$$

uniformly valid in each compact subset $\Omega \subset \bar{D}$, where

$$
\left\{\begin{aligned}
\tilde{d}\left(x, t, w_{0}\right) & =\frac{\partial a}{\partial u}\left(x, t, w_{0}\right) \frac{\partial w_{0}}{\partial t}+\frac{\partial b}{\partial u}\left(x, t, w_{0}\right) \frac{\partial w_{0}}{\partial x}+\frac{\partial d}{\partial u}\left(x, t, w_{0}\right) \\
\Phi_{1}(x, t)= & \frac{\partial^{2} w_{0}}{\partial t^{2}}-c^{2}(x, t) \frac{\partial^{2} w_{0}}{\partial x^{2}} \\
\Psi_{1}(x, \tau)= & \left\{\frac{\partial a}{\partial u}\left(x, 0, w_{0}(x, 0)\right) \frac{\partial w_{0}}{\partial t}(x, 0)+\frac{\partial b}{\partial u}\left(x, 0, w_{0}(x, 0)\right) \frac{\partial w_{0}}{\partial x}(x, 0)\right. \\
& \left.+\frac{\partial d}{\partial u}\left(x, 0, w_{0}(x, 0)\right)\right\} v_{0} \\
& +\left[\left\{\frac{\partial a}{\partial t}\left(x, 0, w_{0}(x, 0)\right)+\frac{\partial a}{\partial u}\left(x, 0, w_{0}(x, 0)\right) \frac{\partial w_{0}}{\partial t}(x, 0)\right\} \tau\right. \\
& \left.+\frac{\partial a}{\partial u}\left(x, 0, w_{0}(x, 0)\right) w_{1}(x, 0)\right] \frac{\partial v_{0}}{\partial \tau} \\
& +b\left(x, 0, w_{0}(x, 0)\right) \frac{\partial v_{0}}{\partial x}+\frac{\partial a}{\partial u}\left(x, 0, w_{0}(x, 0)\right) v_{0} \frac{\partial v_{0}}{\partial \tau} .
\end{aligned}\right.
$$

The functions $w_{k}$ and $v_{k}(k=0,1)$ can now be obtained by solving the following set of initial value problems: 

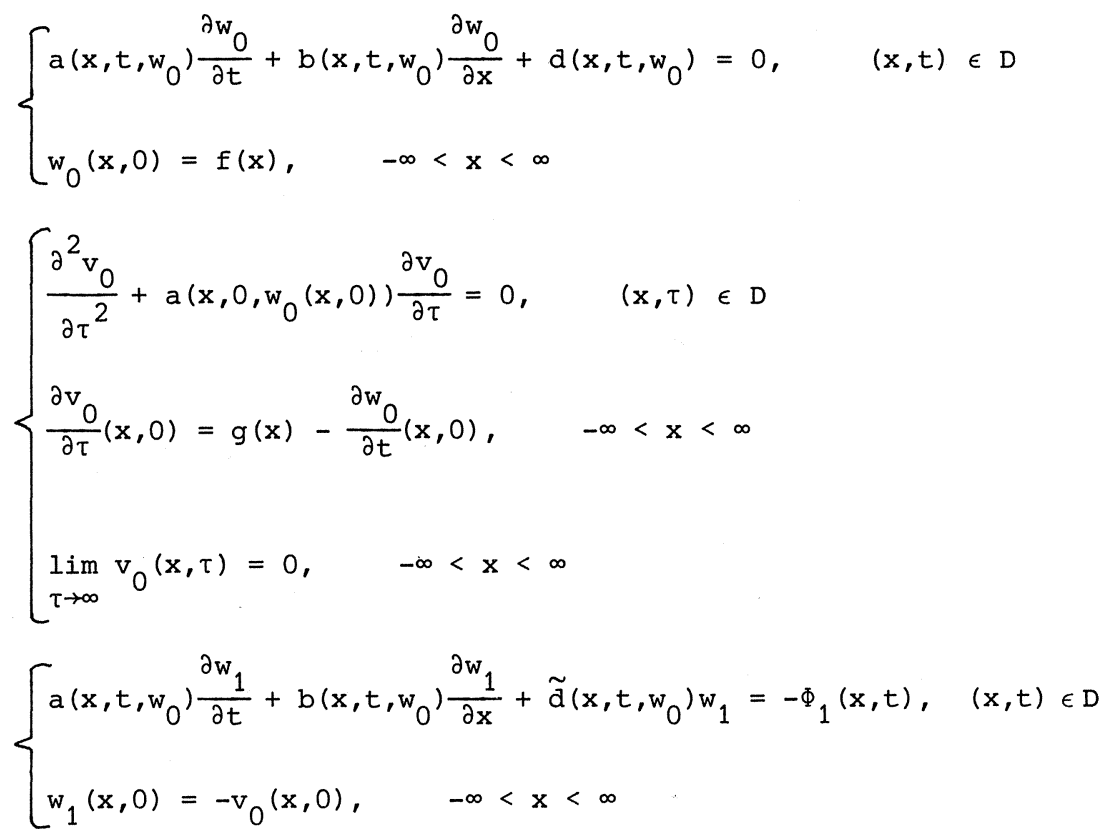

$$
\left\{\begin{array}{l}
\frac{\partial^{2} v_{1}}{\partial \tau^{2}}+a\left(x, 0, w_{0}(x, 0)\right) \frac{\partial v_{1}}{\partial \tau}=-\Psi_{1}(x, \tau), \quad(x, \tau) \in D \\
\frac{\partial v_{1}}{\partial \tau}(x, 0)=-\frac{\partial w_{1}}{\partial t}(x, 0), \quad-\infty<x<\infty \\
\lim _{\tau \rightarrow \infty} v_{1}(x, \tau)=0, \quad-\infty<x<\infty .
\end{array}\right.
$$

Note that only the first approximation $w_{0}$ follows from the solution of a nonlinear problem; the remaining functions $v_{0}, w_{1}$ and $v_{1}$ satisfy linear equations which can easily be solved.

In order to verify the properties (19) we make the crucial assumption

$$
w_{0}(x, t) \in C^{\infty}(\bar{D})
$$

which is only true in case the reduced quasilinear equation (22) is of a very special kind. The regularity of $w_{0}(x, t)$ for general quasilinear first order differential equations will be discussed in section 3 .

It follows from (26) that the conditions (19) are satisfied. In particular, 
the functions $v_{0}$ and $v_{1}$ have the form

$$
\left\{\begin{aligned}
v_{0}(x, \tau)= & \frac{\frac{\partial w_{0}}{\partial t}(x, 0)-g(x)}{a\left(x, 0, w_{0}(x, 0)\right)} \exp \left[-a\left(x, 0, w_{0}(x, 0)\right) \tau\right] \\
v_{1}(x, \tau)= & P_{1}(\tau ; x) \exp \left[-a\left(x, 0, w_{0}(x, 0)\right) \tau\right] \\
& +P_{2}(\tau ; x) \exp \left[-2 a\left(x, 0, w_{0}(x, 0)\right) \tau\right]
\end{aligned}\right.
$$

with $P_{k}(\tau ; x)(k=1,2)$ polynomials in $\tau$ with coefficients depending on $x$. So we see that $v_{0}$ and $v_{1}$ are typical boundary layer functions.

Finally, we obtain from (18), (20), (22), (23), (24) and (25) the result

$$
\left\{\begin{array}{l}
L^{(\varepsilon)}[\tilde{u}]=R(x, t ; \varepsilon), \quad(x, t) \in D \\
\tilde{u}(x, 0 ; \varepsilon)=f(x)+\varepsilon^{2} v_{1}(x, 0), \quad-\infty<x<\infty \\
\tilde{u}_{t}(x, 0 ; \varepsilon)=g(x), \quad-\infty<x<\infty
\end{array}\right.
$$

where $R(x, t ; \varepsilon)$ is some $C^{\infty}$-function satisfying

$$
R(x, t ; \varepsilon)=O\left(\varepsilon^{2}\right), \quad \varepsilon \downarrow 0
$$

uniformly in any compact subset $\Omega \subset \bar{D}$.

Hence the function $\tilde{u}(x, t ; \varepsilon)$ defined by $(18),(21),(22),(23),(24)$ and (25) provides a formal approximation (up to order $\varepsilon^{2}$ ) for the solution $u$ of problem (15).

Higher order approximations can be obtained by considering

$$
\tilde{u}(x, t ; \varepsilon)=\sum_{k=0}^{n} \varepsilon^{k} w_{k}(x, t)+\sum_{k=0}^{n} \varepsilon^{k+1} v_{k}(x, \tau), \quad n \in \mathbb{N}, \quad n \geq 2 .
$$

In that case $w_{k}$ and $v_{k}(k=0,1)$ are given by $(21),(22),(23),(24)$ and (25), whereas $w_{k}$ and $v_{k}(k \geq 2)$ follow from the linear problems 
(28)

$$
\left\{\begin{array}{l}
a\left(x, t, w_{0}\right) \frac{\partial w_{k}}{\partial t}+b\left(x, t, w_{0}\right) \frac{\partial w_{k}}{\partial x}+\tilde{d}\left(x, t, w_{0}\right) w_{k}=-\Phi_{k}(x, t), \quad(x, t) \in D \\
w_{k}(x, 0)=-v_{k-1}(x, 0), \quad-\infty<x<\infty
\end{array}\right.
$$

respectively

$$
\left\{\begin{array}{l}
\frac{\partial^{2} v_{k}}{\partial \tau^{2}}+a\left(x, 0, w_{0}(x, 0)\right) \frac{\partial v_{k}}{\partial \tau}=-\Psi_{k}(x, \tau), \quad(x, \tau) \in D \\
\frac{\partial v_{k}}{\partial \tau}(x, 0)=-\frac{\partial w_{k}}{\partial t}(x, 0), \quad-\infty<x<\infty \\
\lim _{\tau \rightarrow \infty} v_{k}(x, \tau)=0, \quad-\infty<x<\infty
\end{array}\right.
$$

the functions $\Phi_{k}$ and $\Psi_{k}(k \in \mathbb{N}, k \geq 2)$ being completely determined by the functions $\mathrm{w}_{0}, \mathrm{w}_{1}, \ldots, \mathrm{w}_{\mathrm{k}-1}$ respectively the functions $\mathrm{w}_{0}, \mathrm{w}_{1}, \ldots, \mathrm{w}_{\mathrm{k}}$ and $\mathrm{v}_{0}, \mathrm{v}_{1}, \ldots, \mathrm{v}_{\mathrm{k}-1}$. The functions $\mathrm{v}_{\mathrm{k}}(\mathrm{x}, \tau)(\mathrm{k} \in \mathbb{I N} \cup\{0\})$ are boundary layer functions of the type

$$
v_{k}(x, \tau)=\sum_{j=1}^{k+1} P_{j}^{(k)}(\tau ; x) \exp \left[-j a\left(x, 0, w_{0}(x, 0)\right) \tau\right]
$$

with $\mathrm{P}_{j}^{(k)}(\tau ; \mathrm{x})$ a polynomial in $\tau$ with coefficients depending on $\mathrm{x}$.

It follows that

$$
\left\{\begin{array}{l}
\mathrm{u}^{(\varepsilon)}[\tilde{u}]=R(x, t ; \varepsilon), \quad(x, t) \in D \\
\tilde{u}(x, 0 ; \varepsilon)=f(x)+\varepsilon^{n+1} v_{n}(x, 0), \quad-\infty<x<\infty \\
\tilde{u}_{t}(x, 0 ; \varepsilon)=g(x), \quad-\infty<x<\infty
\end{array}\right.
$$

with $R(x, t ; \varepsilon)$ some $C^{\infty}$-function satisfying

$$
R(x, t ; \varepsilon)=O\left(\varepsilon^{n+1}\right), \quad \varepsilon+0
$$

uniformly in any compact subset $\Omega \subset \bar{D}$.

Before entering into a discussion of the condition (26) we shall prove that a formal approximation $\tilde{u}$ is indeed a good approximation for the solution $u$ of (15). To this end we put 


$$
z(x, t ; \varepsilon)=u(x, t ; \varepsilon)-\tilde{u}(x, t ; \varepsilon) .
$$

Then we obtain from (15) and (31)

$$
\begin{aligned}
& {\left[\begin{array}{rl}
\varepsilon\left\{\frac{\partial^{2} z}{\partial t^{2}}-c^{2}(x, t) \frac{\partial^{2} z}{\partial x^{2}}\right\} & +a(x, t, z+\tilde{u}) \frac{\partial z}{\partial t}+b(x, t, z+\tilde{u}) \frac{\partial z}{\partial x} \\
& +\{a(x, t, z+\tilde{u})-a(x, t, \tilde{u})\} \frac{\partial \tilde{u}}{\partial t}
\end{array}\right.} \\
& +\{b(x, t, z+\tilde{u})-b(x, t, \tilde{u})\} \frac{\partial \tilde{u}}{\partial x} \\
& +d(x, t, z+\tilde{u})-d(x, t, \tilde{u})=-R(x, t ; \varepsilon),(x, t) \in D \\
& \begin{array}{l}
z(x, 0 ; \varepsilon)=-\varepsilon^{n+1} v_{n}(x, 0), \quad-\infty<x<\infty \\
z_{t}(x, 0 ; \varepsilon)=0, \quad-\infty<x<\infty .
\end{array}
\end{aligned}
$$

Defining a function $\tilde{z}(x, t ; \varepsilon)$ by

$$
\tilde{z}(x, t ; \varepsilon)=z(x, t ; \varepsilon)+\varepsilon^{n+1} v_{n}(x, 0)
$$

we get

$$
\left\{\begin{aligned}
& \varepsilon\left\{\frac{\partial^{2 \tilde{z}}}{\partial t^{2}}-c^{2}(x, t) \frac{\partial^{2 \tilde{z}}}{\partial x^{2}}\right\}+a^{*}(x, t, \tilde{z} ; \varepsilon) \frac{\partial \tilde{z}}{\partial t}+b^{*}(x, t, \tilde{z} ; \varepsilon) \frac{\partial \tilde{z}}{\partial x} \\
&+d^{*}(x, t, \tilde{z} ; \varepsilon)=-R^{*}(x, t ; \varepsilon), \quad(x, t) \in D \\
& \tilde{z}(x, 0 ; \varepsilon)=\tilde{z}_{t}(x, 0 ; \varepsilon)=0, \quad-\infty<x<\infty
\end{aligned}\right.
$$

where 


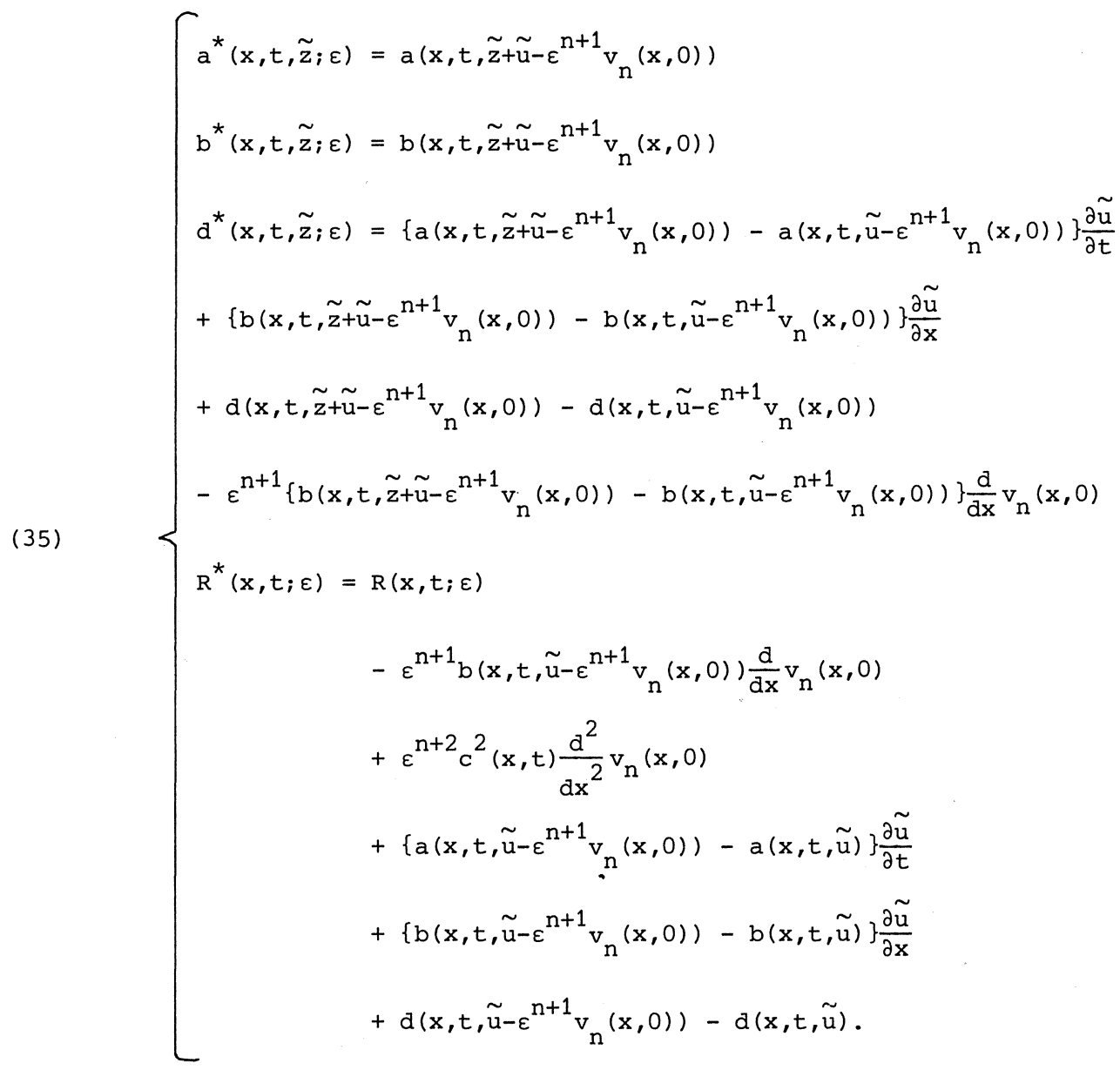

It is easily established that the functions $a^{*}, b^{*}, d^{*}$ and $R^{*}$ satisfy the conditions (7), (9) and (10) of theorem 1 (with Lipschitz exponent $\alpha=1$ ) and that

$$
R^{*}(x, t ; \varepsilon)=O\left(\varepsilon^{n+1}\right), \quad \varepsilon \downarrow 0
$$

uniformly valid in each compact subset $\Omega \subset \bar{D}$.

So taking for $\Omega$ some characteristic region as shown in figure 1 we conclude from theorem 1 that there exists for sufficiently small values of $\varepsilon$ a solution $\tilde{z}$ of (34), provided that (cf. (14))

$$
\mathrm{n}+1>\lambda+\frac{1}{4}
$$


where the constant $\lambda$, according to (13), is given by

$$
\lambda= \begin{cases}\frac{1}{4} & \text { if } a \text { and } b \text { do not depend on } u \\ 1 & \text { otherwise. }\end{cases}
$$

The condition (36) can be simplified to yield $n \geq 0$ (if $a$ and $b$ are independent of $u$ ) or $n \geq 1$ (otherwise).

According to theorem 1 the solution $\tilde{z}$ satisfies the estimate

$$
|\tilde{z}|_{1}^{(\varepsilon)} \leq C(\Omega) \varepsilon^{n+\frac{3}{4}}
$$

where $C(\Omega)$ is some positive constant depending on $\Omega$ but independent of $\varepsilon$. Using (27), (32) and (33) we obtain from (37) the following theorem which is a nonlinear generalization of theorem 2 of chapter III:

THEOREM 2. If the solution $\mathrm{w}_{0}(\mathrm{x}, \mathrm{t})$ of the nonlinear problem (22) belongs to the class $C^{\infty}(\bar{D})$ then there exists for sufficiently small values of $\varepsilon$ a solution $\mathrm{u}$ of problem (15) under the conditions (16) and (17).

This solution and its first derivatives satisfy for any $\mathrm{n} \in \mathbb{I N} \cup\{0\}$ the relations

$$
\begin{cases}u(x, t ; \varepsilon)=\sum_{k=0}^{n} \varepsilon^{k} w_{k}(x, c)+\sum_{k=0}^{n-1} \varepsilon^{k+1} v_{k}(x, \tau)+0\left(\varepsilon^{n+1}\right), & \varepsilon \downarrow 0 \\ \frac{\partial u}{\partial x}(x, t ; \varepsilon)=\sum_{k=0}^{n} \varepsilon^{k} \frac{\partial w_{k}}{\partial x}(x, t)+\sum_{k=0}^{n-1} \varepsilon^{k+1} \frac{\partial v_{k}}{\partial x}(x, \tau)+0\left(\varepsilon^{n+1}\right), & \varepsilon \downarrow 0 \\ \frac{\partial u}{\partial t}(x, t ; \varepsilon)=\sum_{k=0}^{n} \varepsilon^{k} \frac{\partial w_{k}}{\partial t}(x, t)+\sum_{k=0}^{n} \varepsilon^{k} \frac{\partial v_{k}}{\partial \tau}(x, \tau)+0\left(\varepsilon^{n+1}\right), & \varepsilon \downarrow 0\end{cases}
$$

uniformly in any compact subset $\Omega \subset \overline{\mathrm{D}}$; the functions $\mathrm{w}_{\mathrm{k}}$ and $\mathrm{v}_{\mathrm{k}}$ $(\mathrm{k}=0,1, \ldots, \mathrm{n})$ follow successively from $(22),(23),(24),(25),(28)$ and (29) where $\tau$ is defined by $\tau=\frac{t}{\varepsilon}$.

In case $\mathrm{n}=0$ empty sums are to be interpreted as zero.

COROLLARY 1. It follows from the proof of theorem 2 that in case $\mathrm{w}_{0}(\mathrm{x}, \mathrm{t}) \in \mathrm{C}^{\infty}(\Omega)$, with $\Omega$ some characteristic region as shown in figure 1 , the results of theorem 2 remain valid for any compact subset $\Omega^{\prime} \subset \Omega$. 
COROLLARY 2. It follows from (38) that first approximations $(\mathrm{n}=0$ ) for the solution $u$ of (15) and its first derivatives are given by

$$
\left\{\begin{array}{l}
u(x, t ; \varepsilon)=w_{0}(x, t)+0(\varepsilon), \quad \varepsilon \downarrow 0 \\
u_{x}(x, t ; \varepsilon)=\frac{\partial w_{0}}{\partial x}(x, t)+0(\varepsilon), \quad \varepsilon \downarrow 0 \\
u_{t}(x, t ; \varepsilon)=\frac{\partial w_{0}}{\partial t}(x, t)+\left\{g(x)-\frac{\partial w_{0}}{\partial t}(x, 0)\right\} \exp \left[-a\left(x, 0, w_{0}(x, 0)\right) \frac{t}{\varepsilon}\right]+0(\varepsilon), \\
\varepsilon \downarrow 0
\end{array}\right.
$$

uniformly valid in any compact subset $\Omega \subset \overline{\mathrm{D}}$, where $\mathrm{w}_{0}$ is the $\mathrm{C}^{\infty}$-solution of the reduced problem (22).

COROLLARY 3. It follows from (38) and (30) that for any $\mathrm{n} \in \mathbb{I N} \cup\{0\}$

$$
\begin{cases}u(x, t ; \varepsilon)=\sum_{k=0}^{n} \varepsilon^{k} w_{k}(x, t)+0\left(\varepsilon^{n+1}\right), & \varepsilon \downarrow 0 \\ u_{x}(x, t ; \varepsilon)=\sum_{k=0}^{n} \varepsilon^{k} \frac{\partial w_{k}}{\partial x}(x, t)+0\left(\varepsilon^{n+1}\right), & \varepsilon \downarrow 0 \\ u_{t}(x, t ; \varepsilon)=\sum_{k=0}^{n} \varepsilon^{k} \frac{\partial w_{k}}{\partial t}(x, t)+0\left(\varepsilon^{n+1}\right), & \varepsilon \downarrow 0\end{cases}
$$

uniformly valid in any compact subset $\Omega \subset\{(\mathrm{x}, \mathrm{t}) \mid-\infty<\mathrm{x}<\infty, 0<\delta \leq t<\infty\}$ where $\delta$ is an arbitrarily small positive constant independent of $\varepsilon$.

\section{REMARKS.}

1. It is not necessary that the conditions (16) and (17) hold for all $u \in \mathbb{R}$. It is sufficient if these conditions are fulfilled for $u \in I$ with $I$ some open subset of $\mathbb{R}$ containing the range $R_{w_{0}}$ of $w_{0}$. This implies that the functions $a(x, t, u), b(x, t, u)$ and $d(x, t, u)$ are allowed to have singularities in $u$, provided that these singularities are not in $R_{w_{0}}$ (cf. the first remark following theorem 3 in chapter II).

2. The condition of infinite smoothness of the data (and hence also the condition that $w_{0}$ belongs to $\left.C^{\infty}(\bar{D})\right)$ can be weakened. In that case the expansions (38) are valid for all $\mathrm{n} \in \mathbb{I N} \cup\{0\}$ with $\mathrm{n} \leq \mathrm{n}_{0}$, the bound $\mathrm{n}_{0}$ being determined by the differentiability of the data (see also subsection 4.2). 


\section{Generalized solutions of the reduced problem}

In the preceding section we saw that for the construction of a first approximation ( $\mathrm{n}=0$ in theorem 2 ) it is necessary that the solution $\mathrm{w}_{0}$ of the reduced problem belongs at least to the class $c^{2}(\bar{D})$. However, in nonlinear problems of the type (15) where the coefficients $a$ and $b$ depend on $\mathrm{u}$ this necessary condition is often violated, even if the data are $C^{\infty}$-functions. This is caused by the possible multivaluedness of $\mathrm{w}_{0}$, a phenomenon which will now briefly be discussed. For more details we refer to LAX [27].

We look for a solution $u$ of the differential equation

$$
\frac{\partial u}{\partial t}+\Phi(u) \frac{\partial u}{\partial x}=0, \quad(x, t) \in D
$$

which also satisfies the initial condition

$$
u(x, 0)=f(x), \quad-\infty<x<\infty .
$$

We require that

$$
\Phi(u) \in C^{1}(I R), \quad f(x) \in C^{1}(I R) .
$$

It follows from the differential equation (39) that $u$ is constant along characteristics $x=x(t)$ satisfying

(41) $\quad \frac{\mathrm{dx}}{\mathrm{dt}}=\Phi(\mathrm{u})$.

Using the constancy of $u$ we conclude from (41) that the characteristics are straight lines. Geometrically the solution u of (39), (40) can now be constructed by drawing through each point $\left(x_{0}, 0\right)$ a straight line with slope $\frac{d t}{d x}=\frac{1}{\Phi\left(f\left(x_{0}\right)\right)}$. Along this line $u$ has the constant value $f\left(x_{0}\right)$. One easily verifies that for sufficiently small values of $t$ an analytical form of the solution is implicitly given by

$$
u-f(x-t \Phi(u))=0
$$

since we obtain from this formula 
(42)

$$
\left\{\begin{array}{l}
\frac{\partial u}{\partial t}=-\frac{\Phi(u) f^{\prime}(x-t \Phi(u))}{1+t \Phi^{\prime}(u) f^{\prime}(x-t \Phi(u))} \\
\frac{\partial u}{\partial x}=\frac{f^{\prime}(x-t \Phi(u))}{1+t \Phi^{\prime}(u) f^{\prime}(x-t \Phi(u))}
\end{array}\right.
$$

the prime denoting differentiation with respect to the argument.

Let us now assume that $\Phi$ is monotonically increasing, i.e.

$$
\Phi^{\prime}(u)>0 \quad \text { for } u \in \mathbb{R} \text {. }
$$

If $f$ is a monotonically increasing function then it follows from (42) that $\frac{\partial u}{\partial t}$ and $\frac{\partial u}{\partial x}$ remain bounded for all $t>0$. On the other hand, if $f$ is a decreasing function then $\frac{\partial u}{\partial t}$ and $\frac{\partial u}{\partial x}$ tend to infinity as the denominators in (42) approach zero.

This is also illustrated by the geometrical construction mentioned before: for increasing $f$ the characteristics issuing from $t=0$ diverge in the direction of positive $t$ and $u$ remains single-valued for all $t>0$; for decreasing $f$ the characteristics intersect after some time $t=t_{B}$ and $u$ becomes multivalued at the points of intersection (see figure 2).

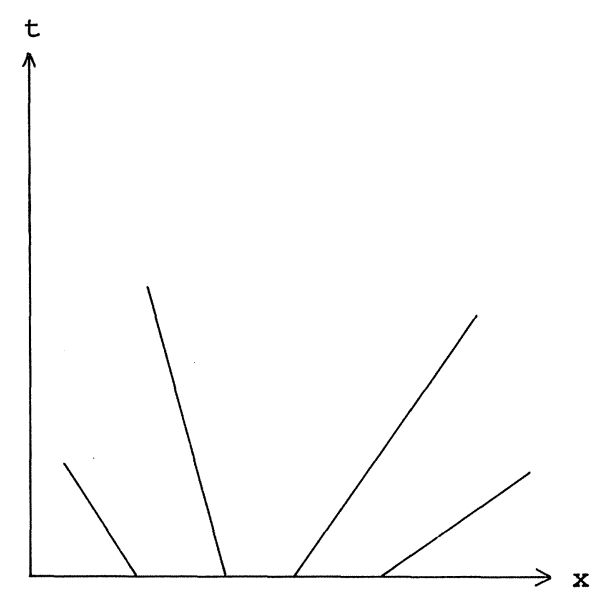

Increasing $f$, diverging characteristics

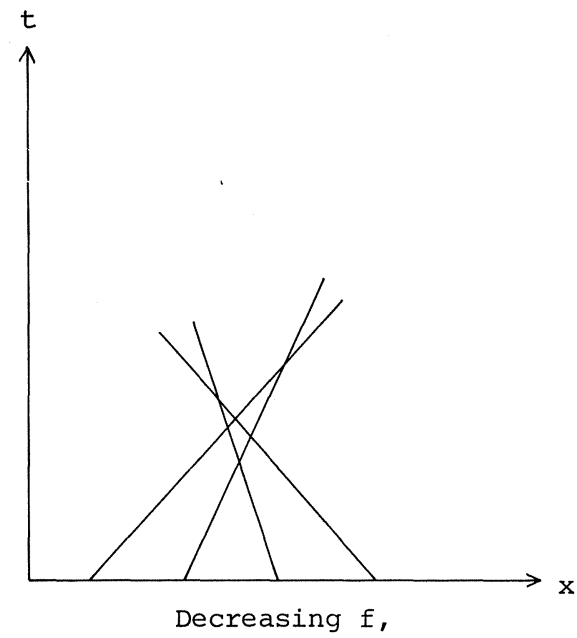

converging characteristics 
We can think of $u$ as a wave with propagation velocity $\Phi(u)$, higher values of $u$ travelling faster than lower ones. If the propagation velocity is a decreasing function of $x$ (which happens for instance if $\Phi^{\prime}(u)>0, f^{\prime}(x)<0$ ) the wave will break after some time $t=t_{B}$ to give a multivalued function $u(x, t)$ (see figure 3 ). It follows that for $t \geq t_{B}$ the derivatives $\frac{\partial u}{\partial t}$ and $\frac{\partial u}{\partial x}$ may become infinite.

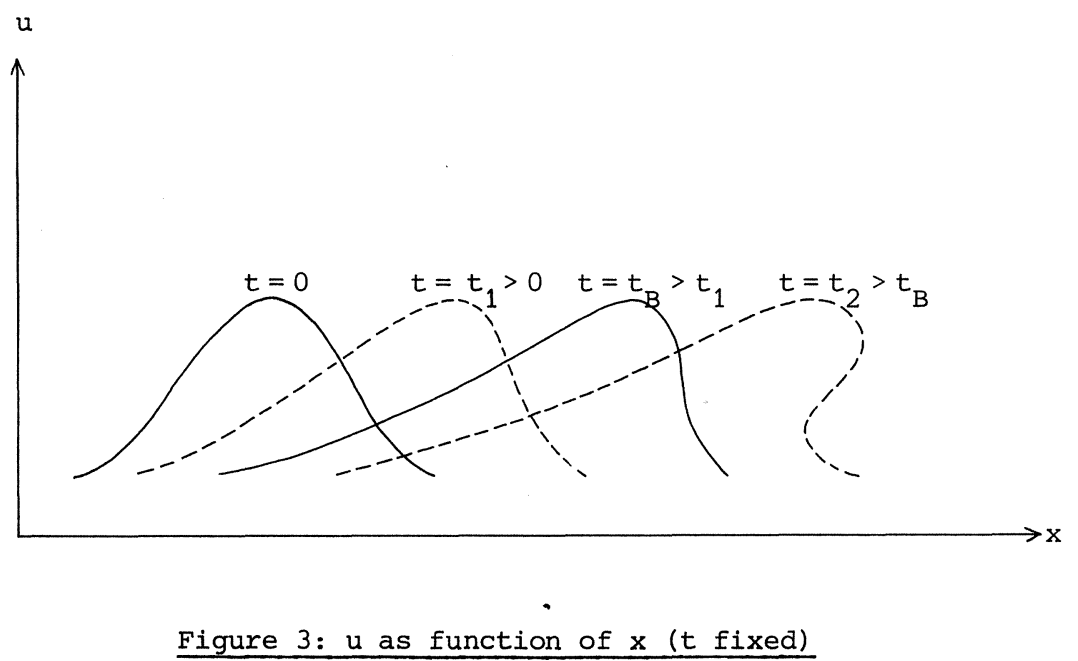

Both from the analytical and the geometrical point of view it will be clear that if the function $f$ is not increasing then no continuous function $u(x, t)$ exists which solves (39), (40) for all $t \geq 0$ in the classical sense. It seems therefore natural to introduce generalized solutions of (39).

DEFINITION 1. A piecewise continuous function $u(x, t)$, continuously differentiable at its points of continuity, is a generalized (distributional, weak) solution of (39) iff

(i) u satisfies (39) in the classical sense at all points of continuity and

(ii) the jump condition

$$
\left(u_{\ell}-u_{r}\right) \frac{d x}{d t}=\int_{u_{r}}^{u_{\ell}} \Phi(\xi) d \xi
$$

holds at all lines of discontinuity $x=x(t)$, where $u_{\ell}$ and $u_{r}$ are defined by 


$$
\left\{\begin{aligned}
u_{\ell}=u_{\ell}(x, t)= & \lim u(y, t) \\
& y \uparrow x \\
u_{r}=u_{r}(x, t)= & \lim _{y \downarrow x} u(y, t) .
\end{aligned}\right.
$$

A generalized solution of (39), (40) with $f$ a piecewise continuous function is not necessarily uniquely determined. However, if we restrict ourselves to generalized solutions which in addition satisfy

$$
u_{\ell}>u_{r}
$$

at every point of discontinuity $(x, t)$ then it can be shown that there exists a uniquely determined (single-valued) function $u(x, t)$ which solves (39), (40) for all $t \geq 0$ in generalized sense. For a discussion of the condition (44) we refer to LAX [27].

The concept of generalized solution can be extended to include bounded measurable functions.

DEFINITION 2. A bounded, measurable function $u(x, t)$ is a generalized (distributional, weak) solution of (39) iff

$$
\iint_{D}\left\{\phi_{t} u+\phi_{x} \int_{0}^{u} \Phi(\xi) d \xi\right\} d x d t=0
$$

for all test functions $\phi \in \mathrm{C}^{1}(\mathrm{D})$ with compact support in $\mathrm{D}$.

It can be shown that for piecewise continuous functions the definitions 1 and 2 are equivalent (cf. LAX [27]).

As before, a generalized solution $u$ of (39), (40) with $f$ a bounded, measurable function is not necessarily uniquely determined. However, also in this case existence and uniqueness can be guaranteed if certain additional conditions are imposed on $u$ (see OLEINIK [33], [35]).

As an illustration we shall give an example of a generalized solution in the sense of definition 1 . 


\section{EXAMPLE 1.}

$$
\left\{\begin{array}{l}
\frac{\partial u}{\partial t}+\arctan u \frac{\partial u}{\partial x}=0, \quad(x, t) \in D \\
u(x, 0)=-x, \quad-\infty<x<\infty .
\end{array}\right.
$$

The solution of this problem is implicitly given by

$$
F(x, t, u) \equiv u-t \arctan u+x=0
$$

and it is easily verified that the lowest value of $t$ for which

$$
F_{u}(x, t, u)=0
$$

is given by $t=1$ (with $x=u=0$ ), so breaking takes place at $t_{B}=1$. This can also be checked graphically. The characteristic through $\left(x_{0}, 0\right)$ is for $t>0$ given by

$$
\arctan x_{0}=\frac{x_{0}-\mathbf{x}}{t}
$$

If we consider (46) as an equation in $x_{0}$ with $x_{0}^{-x}$ and $t$ given then it easily follows from the graphs of arctan $x_{0}$ and $\frac{x_{0}-x}{t}$ that breaking occurs at $(x, t)=(0,1)$ (see figure 4). For $t>1$ and suitably chosen $x$ there are several characteristics passing through $(x, t)$.

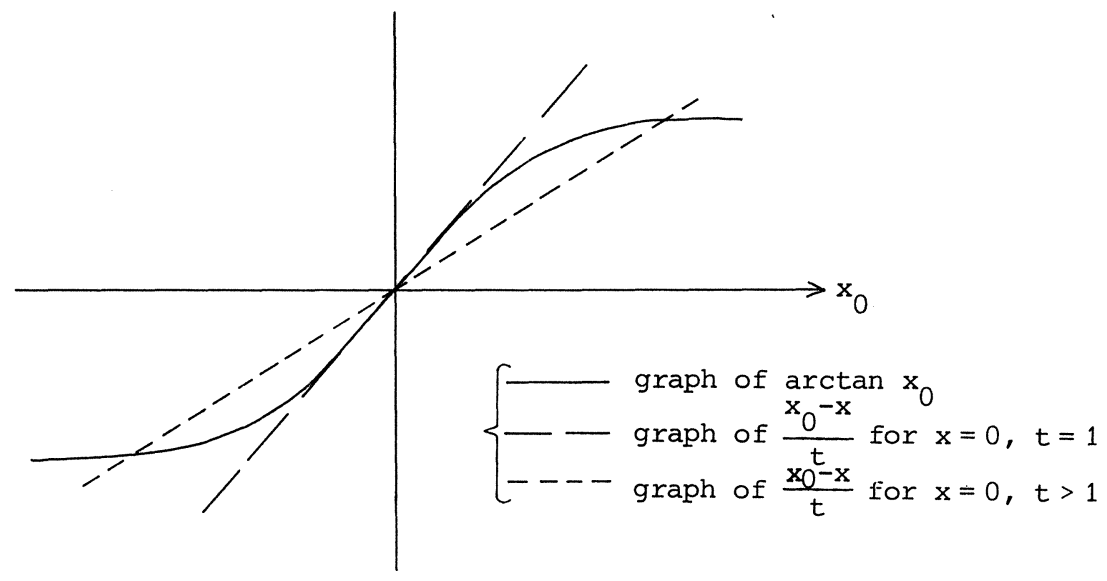

Figure 4 
The region in the $(x, t)$-plane where $u(x, t)$ is multivalued is bounded by the envelope

$$
\left\{\begin{array}{l}
x=\xi-\left(1+\xi^{2}\right) \arctan \xi \\
t=1+\xi^{2}
\end{array} \quad(\xi \in \mathbb{R})\right.
$$

(cf. WHITHAM $[56$, p.23]). We have sketched the situation in figure 5.

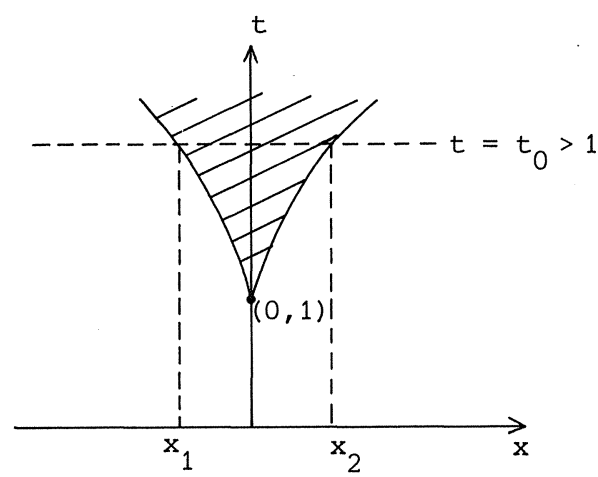

Region of multivaluedness

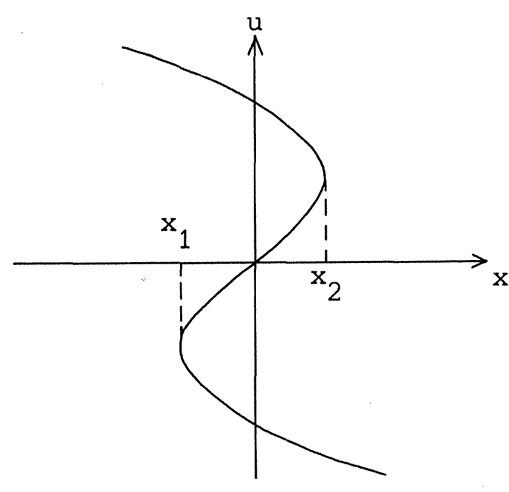

Graph of $u\left(x, t_{0}\right), t_{0}>1$

\section{Figure 5}

In order to obtain from this multivalued function the generalized solution of (45) we have to insert a discontinuity, often referred to as a shock. The line of discontinuity must pass through the point $(0,1)$ and lie in the shaded region (see figure 5). In addition the shock position must be such that the jump condition (43) holds. It is easily verified that the line $\{(x, t) \mid x=0, t \geq 1\}$ satisfies all requirements because of the uneven character of the problem. The generalized piecewise continuous solution is sketched in figure 6 . 


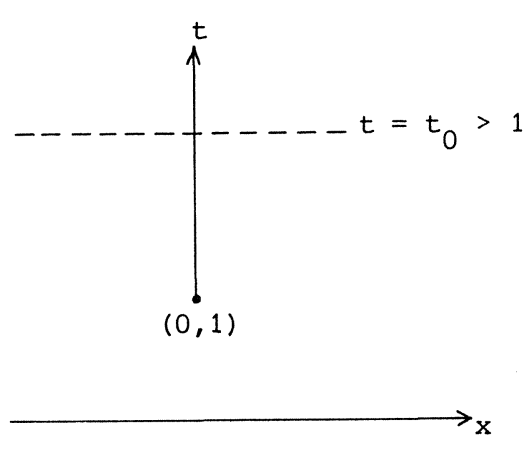

Shock position

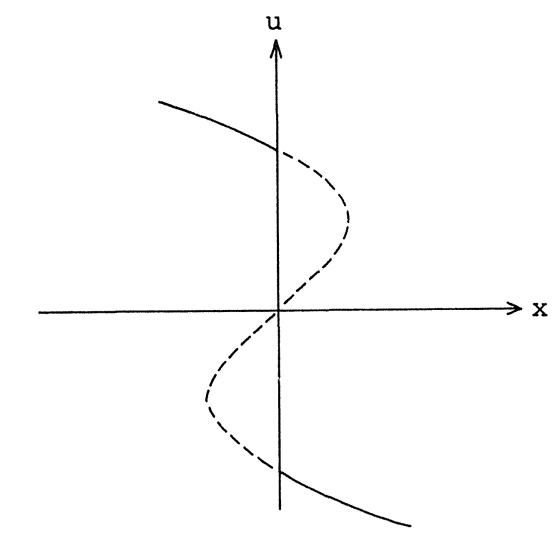

Generalized solution at $t=t_{0}>1$

\section{Figure 6}

From this example one might get the impression that the line of discontinuity necessarily coincides with the characteristic through the breaking point. However, this is not true, as an analysis of the following problem shows:

$$
\begin{cases}\frac{\partial u}{\partial t}+\arctan u \frac{\partial u}{\partial x}=0, & (x, t) \in D \\ u(x, 0)= \begin{cases}-x-\frac{1}{3} x^{3}, & x \geq 0 \\ -x, & x<0 .\end{cases} \end{cases}
$$

The breaking point is again given by $(0,1)$ but one may verify that the characteristic $\mathrm{x}=0$ does not satisfy the jump condition (43), so it cannot coincide with the line of discontinuity.

Finally, it should be remarked that the concept of generalized solutions can be extended to more general initial value problems of the type

$$
\left\{\begin{array}{l}
\frac{\partial u}{\partial t}+\Phi(x, t, u) \frac{\partial u}{\partial x}+\Psi(x, t, u)=0, \quad(x, t) \in D \\
u(x, 0)=f(x), \quad-\infty<x<\infty .
\end{array}\right.
$$


Also the restriction that $\Phi$ be monotonic in $u$ can be disposed of (see OLEINIK [36]).

Let us now return to singular perturbation problems and see what the introduction of generalized solutions leads to. For singular perturbations of parabolic type it is known (OLEINIK [33], [34]) that under appropriate conditions on the data the solution $u$ of

$$
\left\{\begin{array}{l}
\varepsilon \frac{\partial^{2} u}{\partial x^{2}}=\frac{\partial u}{\partial t}+\Phi(x, t, u) \frac{\partial u}{\partial x}+\Psi(x, t, u), \quad(x, t) \in D \\
u(x, 0 ; \varepsilon)=f(x), \quad-\infty<x<\infty
\end{array}\right.
$$

converges for $\varepsilon+0$ in some integral.norm to the generalized solution $w$ of

$$
\left\{\begin{array}{l}
\frac{\partial w}{\partial t}+\Phi(x, t, w) \frac{\partial w}{\partial x}+\Psi(x, t, w)=0, \quad(x, t) \in D \\
w(x, 0)=f(x), \quad-\infty<x<\infty
\end{array}\right.
$$

More strongly, if $\Phi(x, t, u)=u$ and $\Psi(x, t, u) \equiv 0$ we have BURGERS' equation and problem (48) can be solved explicitly -(see BURGERS [5] and WHITHAM [56, chapter 4$]$ ). It turns out that $u$ converges pointwise to $w$ for $\varepsilon \downarrow 0$ with the exception of arbitrarily small neighborhoods of the lines of discontinuity where a smooth transition takes place (see figure 7).
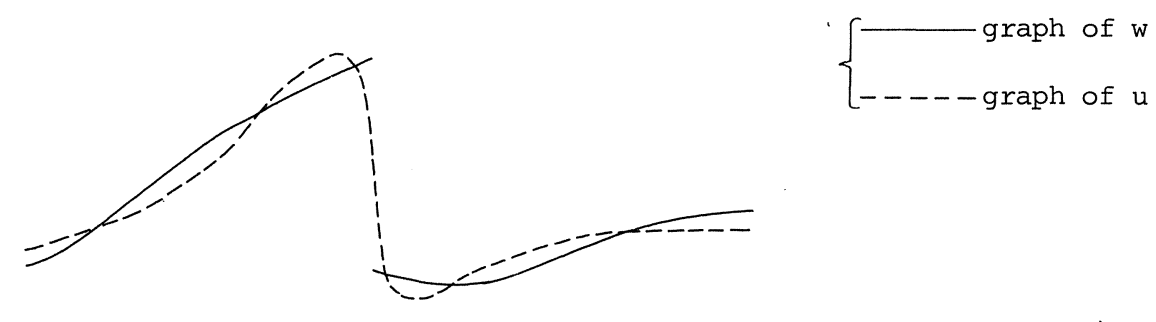
We strongly believe that similar results hold for problems with a singular perturbation of hyperbolic type. A possible strategy to solve this kind of problems will now be illustrated by the following example

$$
\left\{\begin{array}{l}
L^{(\varepsilon)}[u] \equiv \varepsilon\left(\frac{\partial^{2} u}{\partial t^{2}}-c^{2} \frac{\partial^{2} u}{\partial x^{2}}\right)+\frac{\partial u}{\partial t}+\arctan u \frac{\partial u}{\partial x}=0, \\
u(x, 0 ; \varepsilon)=-x, \quad-\infty<x<\infty \\
u_{t}(x, 0 ; \varepsilon)=0, \quad-\infty<x<\infty
\end{array}\right.
$$

where $\mathrm{c}$ is some constant satisfying

$$
c \geq \frac{\pi}{2}
$$

This inequality implies that the timelike condition is fulfilled.

From example 1 we know the shock position and the region of multivaluedness for the solution of the reduced problem. We draw through the breaking point $(0,1)$ two characteristics satisfying $\frac{d x}{d t}= \pm c$. Using the timelike condition (49) it can be shown that these characteristics lie outside the region of multivaluedness (see (47) and figure 8 ).

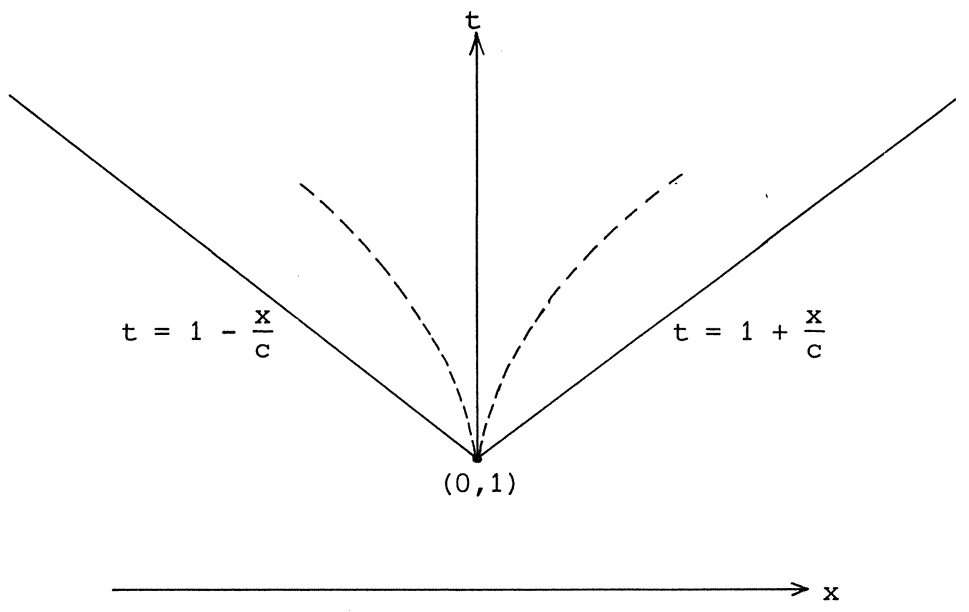

\section{Figure 8}

Since the generalized solution of the reduced problem is infinitely smooth in the region 


$$
D^{*}=\left\{(x, t) \mid 0 \leq t<1+\frac{x}{c} v 0 \leq t<1-\frac{x}{c}\right\}
$$

we have the approximation

$$
u(x, t ; \varepsilon)=w(x, t)+0(\varepsilon), \quad \varepsilon+0
$$

uniformly valid in each compact subset $\Omega \subset D^{*}$ (see corollary 1 of theorem 2 ). Approximations for $u$ in the remaining region $D \backslash D$ * have not been established yet. Since it follows from (43) that shocks originating from the reduced equation necessarily take place along timelike curves in the $(x, t)-p l a n e ~ a$ promising strategy for future investigations seems to be the following (see also chapter VI) :

1. Define for an arbitrarily small positive number $\delta$ (independent of $\varepsilon$ ) the region $\mathrm{D}_{\delta}$ by

$$
D_{\delta}=\left\{(x, t) \mid x>0 \wedge t>1+\frac{x}{C}-\delta\right\}
$$

and consider the Goursat problem $\mathrm{L}^{(\varepsilon)}\left[\mathrm{u}_{\mathrm{l}}\right]=0$ in $\mathrm{D}_{\delta^{\prime}}$ assuming the values of $\mathrm{u}$ along the boundary to be known.

2. Construct a formal approximation $\tilde{u}$ for $u$ in $\bar{D}_{\delta}$, consisting of the generalized solution $\mathrm{w}$ augmented with a boundary layer function at $\mathrm{x}=0$.

3. Prove that $\lim \{u(x, t ; \varepsilon)-\tilde{u}(x, t ; \varepsilon)\}=0$ in $\bar{D}_{\delta}$, using the fact that $\varepsilon \downarrow 0$

$$
u\left(x, 1+\frac{x}{c}-\delta ; \varepsilon\right)-w\left(x, 1+\frac{x}{c}-\delta\right)=0(\varepsilon), \quad \varepsilon+0 \text { for } x \geq 0
$$

(cf. chapter VI).

Since the unknown function $u(0, t ; \varepsilon), t \geq 1$ occurs only in the boundary layer function it follows that

$$
\lim _{\varepsilon \downarrow 0} u(x, t ; \varepsilon)=w(x, t) \text { in }\left\{(x, t) \mid x \geq \delta \wedge t \geq 1+\frac{x}{c}-\delta\right\} .
$$

In a similar way one obtains an estimate in $\left\{(x, t) \mid x \leq-\delta \wedge t \geq 1-\frac{x}{c}-\delta\right\}$. The above mentioned procedure can be carried out if one knows a priori that both $u(x, t ; \varepsilon)$ and its tangential derivative along the shock remain bounded 
as $\varepsilon$ tends to zero. Finally, approximations in regions containing a part of the line along which the shock takes place may be constructed by adding to the generalized solution a free boundary layer giving a smooth transition (depending on $\varepsilon$ ) from values of $w(x, t)$ with $x<0$ to values of $w(x, t)$ with $x>0$.

In the next section we shall discuss two nonlinear initial value problems of the type (15) in which the coefficients $a$ and $b$ are independent of u.

\section{Problems with $\mathrm{a}$ and $\mathrm{b}$ independent of $\mathrm{u}$}

\subsection{The case $d(x, t, u)=d(x, t) \sin u$}

We consider the quasilinear initial value problem

(50)

$$
\begin{cases}\varepsilon\left\{\frac{\partial^{2} u}{\partial t^{2}}-c^{2}(x, t) \frac{\partial^{2} u}{\partial x^{2}}\right\}+a(x, t) \frac{\partial u}{\partial t}+b(x, t) \frac{\partial u}{\partial x}+d(x, t) \sin u=0, & (x, t) \in D \\ u(x, 0 ; \varepsilon)=f(x), \quad-c 2 x<\infty & -\infty<x<\infty\end{cases}
$$

where $\varepsilon$ is a small positive parameter, i.e. $0<\varepsilon<1$.

The following conditions are imposed on the data

(51)

$$
\left\{\begin{array}{l}
c(x, t) \in C^{\infty}(\bar{D}), \quad d(x, t) \in C^{\infty}(\bar{D}) \\
f(x) \in C^{\infty}(I R), \quad g(x) \in C^{\infty}(I R) \\
C(x, t)>0 \text { in } \bar{D} \\
C(x, t) \text { is uniformly bounded in any strip } \\
\{(x, t) \mid-\infty<x<\infty, 0 \leq t \leq T\}
\end{array}\right.
$$

and 


$$
\left\{\begin{array}{l}
a(x, t) \in C^{\infty}(\bar{D}), \quad b(x, t) \in C^{\infty}(\bar{D}) \\
a(x, t)>0 \quad \text { in } \bar{D} \\
|b(x, t)|<a(x, t) c(x, t) \quad \text { in } \bar{D} .
\end{array}\right.
$$

We ask for an approximation of the solution $u$ of (50), valid for small values of $\varepsilon$.

It has been shown in section 2 that the reduced problem

$$
\begin{cases}a(x, t) \frac{\partial w}{\partial t}+b(x, t) \frac{\partial w}{\partial x}+d(x, t) \sin w=0, & (x, t) \in D \\ w(x, 0)=f(x), \quad-\infty<x<\infty & \end{cases}
$$

holds a key position in the construction of an approximation for the solution $u$ of (50); it is in fact the smoothness of the solution w of (52) which determines the highest order of accuracy we can obtain. Since an obvious change of coordinates transforms problem (52) into an equivalent problem of the type

$$
\left\{\begin{array}{l}
\frac{\partial w}{\partial t}+d(x, t) \sin w=0, \quad(x, t) \in D \\
w(x, 0)=f(x), \quad-\infty<x<\infty
\end{array}\right.
$$

it follows that instead of (50) we may consider without loss of generality the simpler problem

$$
\begin{cases}\varepsilon\left\{\frac{\partial^{2} u}{\partial t^{2}}-c^{2}(x, t) \frac{\partial^{2} u}{\partial x^{2}}\right\}+\frac{\partial u}{\partial t}+d(x, t) \sin u=0, & (x, t) \in D \\ u(x, 0 ; \varepsilon)=f(x), & -\infty<x<\infty \\ u_{t}(x, 0 ; \varepsilon)=g(x), \quad-\infty<x<\infty\end{cases}
$$

for as far as the construction of an approximation for $u$ is concerned there is qualitatively no difference between the problems (50) and (54).

An approximation for the solution $u$ of (54) can easily be given in explicit form. We find for the solution w of the reduced problem (53) 


$$
\left\{\begin{aligned}
w(x, t)=2 \arctan \left[\tan \left\{\frac{1}{2} f(x)\right\} \exp \{\phi(x, t)\}\right]+2 k \pi \\
\text { if }(x, t) \in A_{k^{\prime}} k \in \mathbb{Z} \\
w(x, t)=(2 k+1) \pi \quad \text { if }(x, t) \in B_{k^{\prime}} k \in \mathbb{Z}
\end{aligned}\right.
$$

where

$$
\left\{\begin{array}{l}
A_{k}=\{(x, t) \mid(2 k-1) \pi<f(x)<(2 k+1) \pi, 0 \leq t<\infty\}, \quad k \in \mathbb{Z} \\
B_{k}=\{(x, t) \mid f(x)=(2 k+1) \pi, 0 \leq t<\infty\}, \quad k \in \mathbb{Z} \\
\phi(x, t)=-\int_{0}^{t} d(x, \tau) d \tau .
\end{array}\right.
$$

Since $w(x, t) \in C^{\infty}(\bar{D})$ we obtain from corollary 2 of theorem 2

THEOREM 3. For sufficiently small values of $\varepsilon$ there exists a solution $u$ of problem (54) under the conditions (51). This solution satisfies

$$
u(x, t ; \varepsilon)=w(x, t)+O(\varepsilon), \quad \varepsilon \downarrow 0
$$

uniformly in each compact subset $\Omega \subset \dot{\bar{D}}$, where $\mathrm{w}(\mathrm{x}, \mathrm{t})$ is given by (55) and (56).

A sketch of $w$ as function of $x$ for different values of $t$ is given in figure 9 .

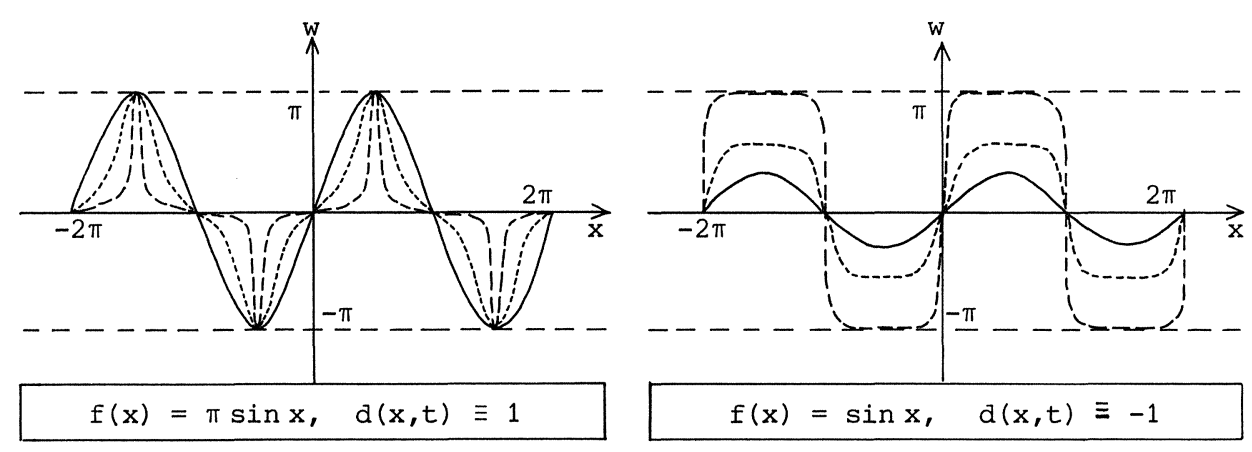

$$
\text { Figure 9: } w \text { as function of } x \text { for }\left\{\begin{array}{l}
t=0 \\
t=t_{1}>0 \\
t=t_{2}>t_{1} \ldots-\ldots
\end{array}\right.
$$


Note that in case $d(x, t) \equiv 1$

$$
\lim _{t \rightarrow \infty} w(x, t)=2 k \pi \quad \text { if } \quad(2 k-1) \pi<f(x)<(2 k+1) \pi, \quad k \in \mathbb{Z}
$$

and in case $d(x, t) \equiv-1$

$$
\lim _{t \rightarrow \infty} w(x, t)=\left\{\begin{array}{llll}
(2 k+1) \pi & \text { if } & 2 k \pi<f(x)<(2 k+1) \pi, & k \in \mathbb{Z} \\
(2 k-1) \pi & \text { if } & (2 k-1) \pi<f(x)<2 k \pi, & k \in \mathbb{Z} .
\end{array}\right.
$$

\subsection{The case $d(x, t, u)=d_{1}(x, t) u+d_{2}(x, t) u|u|^{\nu}$}

We consider the quasilinear initial value problem

(57)

$$
\left\{\begin{aligned}
\varepsilon\left\{\frac{\partial^{2} u}{\partial t^{2}}-c^{2}(x, t) \frac{\partial^{2} u}{\partial x^{2}}\right\} & +a(x, t) \frac{\partial u}{\partial t}+b(x, t) \frac{\partial u}{\partial x} \\
& +d_{1}(x, t) u+d_{2}(x, t) u|u|^{\nu}=0, \quad(x, t) \in D \\
u(x, 0 ; \varepsilon)=f(x), & -\infty<x \leq \infty \\
u_{t}(x, 0 ; \varepsilon)=g(x), & -\infty<x<\infty
\end{aligned}\right.
$$

where $\varepsilon$ is a small positive parameter, i.e. $0<\varepsilon<1$, and $\nu$ is a positive number.

The following conditions are imposed on the data

$$
\left\{\begin{array}{l}
c(x, t) \in C^{\infty}(\bar{D}), \quad f(x) \in C^{\infty}(\mathbb{R}), \quad g(x) \in C^{\infty}(\mathbb{R}) \\
c(x, t)>0 \text { in } \bar{D} \\
c(x, t) \text { is uniformly bounded in any strip } \\
\{(x, t) \mid-\infty<x<\infty, 0 \leq t \leq T\}
\end{array}\right.
$$

and 


$$
\left\{\begin{array}{l}
a(x, t) \in C^{\infty}(\bar{D}), \quad b(x, t) \in C^{\infty}(\bar{D}) \\
a_{1}(x, t) \in C^{\infty}(\bar{D}), \quad d_{2}(x, t) \in C^{\infty}(\bar{D}) \\
a(x, t)>0 \text { in } \bar{D}, \quad d_{2}(x, t)>0 \text { in } \bar{D} \\
|b(x, t)|<a(x, t) c(x, t) \quad \text { in } \bar{D} .
\end{array}\right.
$$

We ask for an approximation of the solution $u$ of (57), valid for small values of $\varepsilon$. Note that in this problem the function $d(x, t, u)$ does not belong to the class $C^{\infty}(\bar{D} \times I R)$, except when $v$ is even.

In order not to complicate the calculations we shall restrict ourselves to the following special form of problem (57):

$$
\begin{cases}L^{(\varepsilon)}[u] \equiv \varepsilon\left\{\frac{\partial^{2} u}{\partial t^{2}}-c^{2}(x, t) \frac{\partial^{2} u}{\partial x^{2}}\right\}+\frac{\partial u}{\partial t}+u|u|^{\nu}=0, & (x, t) \in D \\ u(x, 0 ; \varepsilon)=f(x), & -\infty<x<\infty \\ u_{t}(x, 0 ; \varepsilon)=g(x), & -\infty<x<\infty .\end{cases}
$$

As we pointed out in subsection 4.1 this is no serious limitation as long as we are concerned with approximations of $u$, since the reduced problem

$$
\left\{\begin{array}{l}
a(x, t) \frac{\partial w}{\partial t}+b(x, t) \frac{\partial w}{\partial x}+d_{1}(x, t) w+d_{2}(x, t) w|w|^{\nu}=0, \quad(x, t) \in D \\
w(x, 0)=f(x), \quad-\infty<x<\infty
\end{array}\right.
$$

can always be transformed into an equivalent problem of the type

$$
\begin{cases}\frac{\partial w}{\partial t}+w|w|^{\nu}=0, & (x, t) \in D \\ w(x, 0)=f(x), & -\infty<x<\infty\end{cases}
$$

by means of a LIOUVILLE transformation followed by a transition to characteristic coordinates.

We shall now establish an approximation for the solution $u$ of (59).

Firstly, let us remark that for even values of $v$ the theory of section 2 
may be applied in a straightforward manner, since we have in that case $d(x, t, u) \in C^{\infty}(\bar{D} \times I R)$ and the solution $w$ of $(60)$, given by

$$
w(x, t)=\frac{f(x)}{\left\{1+\nu t|f(x)|^{\nu}\right\}^{1 / \nu}}
$$

belongs to $C^{\infty}(\bar{D})$. So we obtain from corollary 2 of theorem 2

$$
u(x, t ; \varepsilon)=\frac{f(x)}{\left\{1+\nu t|f(x)|^{\nu}\right\}^{1 / \nu}}+o(\varepsilon), \quad \varepsilon+0
$$

for even $\nu$, uniformly valid in each compact subset $\Omega \subset \bar{D}$.

In order to deal with other values of $\nu$ we shall assume for the moment that the function $f$ has no zeroes. Then the solution (61) of the reduced problem belongs again to $C^{\infty}(\bar{D})$. Starting with this function as a first approximation $w_{0}$ it is possible to construct for any $n \in \mathbb{N} \cup\{0\}$ a formal approximation $\tilde{u}$ for $u$ in the form

$$
\tilde{u}(x, t ; \varepsilon)=\sum_{k=0}^{n} \varepsilon^{k} w_{k}(x, t)+\sum_{k=0}^{n} \varepsilon^{k+1} v_{k}(x, \tau)
$$

in the same way as we did in section 2. Note that the higher derivatives of the function $u|u|^{\nu}$ remain bounded for $u$ in a neighborhood of $w_{0}$ due to the fact that $w_{0}$ has no zeroes.

The proof of the correctness of the expansion is similar to the proof given in section 2. The only difference is that in the application of theorem 1 to problem (34) the Lipschitz exponent $\alpha$ mentioned in $(10$, iii) is now given by

$$
\alpha=\min (\nu, 1)
$$

instead of $\alpha=1$, since the function $d^{*}(x, t, \tilde{z} ; \varepsilon)$ defined by (35) satisfies in this case

$$
\frac{\partial d^{*}}{\partial \tilde{z}}(x, t, \tilde{z} ; \varepsilon)=(1+\nu)\left|\tilde{z}+\tilde{u}-\varepsilon^{n+1} v_{n}(x, 0)\right|^{\nu}
$$

As in section 2 the application of theorem 1 to problem (34) leads to the existence of a solution $\tilde{z}$ satisfying (37), provided that (cf. (14)) 


$$
\mathrm{n}+1>\lambda+\frac{1}{4}
$$

Here the constant $\lambda$, according to (13), is given by

$$
\lambda=\frac{1}{4 \min (\nu, 1)} .
$$

The condition (63) can also be written as

$$
\min (\nu, 1)>\frac{1}{4 n+3}
$$

Hence we may conclude that an increase of the number of terms in (62) yields not only better approximations for $u$ but it also enables us to consider smaller values of $\nu$. In particular, letting $n$ tend to infinity we obtain for all $\nu>0$

$$
u(x, t ; \varepsilon)=\frac{f(x)}{\left\{1+\nu t|f(x)|^{\nu}\right\}^{1 / \nu}}+O(\varepsilon), \quad \varepsilon+0
$$

uniformly valid in each compact subset $\Omega \subset \bar{D}$, provided that $f$ has no zeroes. Let us now investigate what happens if the function $f$ has zeroes. Starting with (61) as a first approximation $w_{0}$ it is not possible to construct a formal approximation of arbitrarily high order, because for any fixed value of $v$

(i) the higher derivatives of $u|u|^{\nu}$ may become unbounded in a neighborhood of $w_{0}$, since $w_{0}$ may be equal to zero,

(ii) the higher derivatives of $\mathrm{w}_{0}$ with respect to $\mathrm{x}$ become unbounded in a neighborhood of a zero of $f$.

As for the derivatives of $w_{0}$, a calculation shows that

$$
\left\{\begin{array}{l}
\frac{\partial w_{0}}{\partial x}=\frac{f^{\prime}(x)}{\left\{1+v t|f(x)|^{\nu}\right\}^{1+1 / \nu}} \\
\frac{\partial^{2} w_{0}}{\partial x^{2}}=\frac{f^{\prime \prime}(x)}{\left\{1+v t|f(x)|^{\nu}\right\}^{1+1 / \nu}}-\frac{v(\nu+1) t|f(x)|^{\nu}\left\{f^{\prime}(x)\right\}^{2}}{f(x)\left\{1+v t|f(x)|^{\nu}\right\}^{2+1 / v}}
\end{array}\right.
$$


so if $f$ has in $x=x_{0}$ a zero with multiplicity $k(k \in \mathbb{N})$ then it follows that in a neighborhood of $x=x_{0}$

N.B1. $\frac{\partial^{2} w_{0}}{\partial x^{2}}$ has a jump discontinuity in $x=x_{0}$ if $v=k=1$.

$$
\frac{\partial^{2} w_{0}}{\partial x^{2}} \text { is }\left\{\begin{array}{l}
\text { unbounded if } 0<v<1, k=1 \\
\text { bounded otherwise. }
\end{array}\right.
$$

N.B2. If $f(x) \equiv 0$ in a neighborhood of $x=x_{0}$ then we can think of $x_{0}$ as a zero with infinite multiplicity.

On the other hand, $w_{0}$ is for all positive values of $\nu$ infinitely continuously differentiable with respect to $t$.

From the fact that $\frac{\partial^{2} w_{0}}{\partial x^{2}}$ is unbounded for $0<\nu<1, k=1$ it apparently follows that for small values of $v$ we cannot even start the formal approximation with a first term $w_{0}$ given by (61). Although it will turn out that we can do something about this by some regularization technique it will be clear that we may not expect to obtain expansions of high order. Of course, the highest possible order of the approximation increases as $\nu$ increases, but since we want our results to be valid for as many values of $v$ as possible, including small ones, we shall from now on restrict ourselves to first order approximations. Putting

$$
\begin{aligned}
\tilde{u}(x, t ; \varepsilon) & =w_{0}(x, t)+\varepsilon v_{0}\left(x, \frac{t}{\varepsilon}\right) \\
& =\frac{f(x)}{\left\{1+\nu t|f(x)|^{\nu}\right\}^{1 / \nu}}-\varepsilon\left\{g(x)+f(x)|f(x)|^{\nu}\right\} \exp \left(-\frac{t}{\varepsilon}\right)
\end{aligned}
$$

and excluding for a moment the combination $0<\nu<1, k=1$ we obtain as in section 2 (formula (31) with $\mathrm{n}=0$ )

$$
\left\{\begin{array}{l}
L^{(\varepsilon)}[\tilde{u}]=R(x, t ; \varepsilon), \quad(x, t) \in D \\
\tilde{u}(x, 0 ; \varepsilon)=f(x)-\varepsilon\left\{g(x)+f(x)|f(x)|^{\nu}\right\}, \quad-\infty<x<\infty \\
\tilde{u}_{t}(x, 0 ; \varepsilon)=g(x), \quad-\infty<x<\infty
\end{array}\right.
$$


where $R(x, t ; \varepsilon)$ is a function satisfying

$$
\mathrm{R}(\mathrm{x}, \mathrm{t} ; \varepsilon)=0(\varepsilon), \quad \varepsilon \downarrow 0
$$

uniformly in each compact subset $\Omega \subset \overline{\mathrm{D}}$.

Applying the estimate (37) with $\mathrm{n}=0$ we get

$$
u(x, t ; \varepsilon)=\frac{f(x)}{\left\{1+\nu t|f(x)|^{\nu}\right\}^{1 / \nu}}+0\left(\varepsilon^{\frac{3}{4}}\right), \quad \varepsilon \downarrow 0
$$

uniformly valid in each compact subset $\Omega \subset \bar{D}$, provided that (64) holds with $\mathrm{n}=0$, that is

$$
\nu>\frac{1}{3}
$$

Note that for $0<v \leq \frac{1}{3}, k>1$ approximations of higher order are required because of (64); in that case one must also investigate the regularity of the functions $\frac{\partial^{n_{w_{0}}}}{\partial x^{n}}(n \geq 3)$. Since this involves a number of tedious calculations which do not lead to an essential improvement of the results we shall not go further into this matter.

We shall now examine the case $0<1<1, \mathrm{k}=1$.

Let us suppose that $f$ has a certain number of zeroes with multiplicity $k=1$ which are nowhere dense in IR. Since we only consider approximations in bounded subsets of $\bar{D}$ we may assume without loss of generality that this number is finite, say $m$, and we denote the zeroes by $x_{i}(i=1,2, \ldots, m)$ where $x_{i}<x_{j} f_{\partial}{ }^{r} i<j$. Since $\frac{\partial^{2} w_{0}}{\partial x^{2}}$ is unbounded in a neighborhood of each of the zeroes we shall consider instead of (59) the nodified problem

$$
\left\{\begin{array}{l}
\varepsilon\left\{\frac{\partial^{2} U}{\partial t^{2}}-c^{2}(x, t) \frac{\partial^{2} U}{\partial x^{2}}\right\}+\frac{\partial U}{\partial t}+U|U|^{\nu}=0, \quad(x, t) \in D \\
U(x, 0 ; \varepsilon)=\tilde{f}(x ; \varepsilon), \quad-\infty<x<\infty \\
U_{t}(x, 0 ; \varepsilon)=g(x), \quad-\infty<x<\infty
\end{array}\right.
$$

with $0<\nu<1$ and

$$
\tilde{f}(x ; \varepsilon)=f(x) \prod_{i=1}^{m}\left[1-\exp \left\{-\frac{\left(x-x_{i}\right)^{2}}{\varepsilon^{2 p}}\right\} .\right.
$$


Here $\mathrm{p}$ is some positive constant to be determined later on.

Note that the zeroes of $\tilde{\mathrm{f}}$ have multiplicity $\mathrm{k} \geq 2$. Moreover, we obtain from (67)

$$
f(x)-\tilde{f}(x ; \varepsilon)=f(x)\left[1-\prod_{i=1}^{m}\left[1-\exp \left\{-\frac{\left(x-x_{i}\right)^{2}}{\varepsilon^{2 p}}\right\}\right]\right.
$$

$$
f(x) \sum_{i=1}^{m} \exp \left\{-\frac{\left(x-x_{i}\right)^{2}}{\varepsilon^{2 p}}\right\}+o\left(\varepsilon^{N}\right), \quad \varepsilon \downarrow 0
$$

uniformly valid in every compact subset of $I R$, where $N$ is an arbitrarily large positive number. Since $x_{i}(i=1,2, \ldots, m)$ is a zero of $f$ with multiplicity $k=1$ it follows that

$$
f(x)-\tilde{f}(x ; \varepsilon)=0\left(\varepsilon^{p}\right), \quad \varepsilon \downarrow 0
$$

uniformly valid in every compact subset of IR.

For small values of $\varepsilon$ the initial functions $f$ and $\tilde{E}$ differ only slightly, so we may expect the solution $U$ of (66) to differ slightly from the solution u of (59). We shall now try to approximate u by constructing an approximation for $\mathrm{U}$.

A first approximation for $U$ is given by

$$
\tilde{U}(x, t ; \varepsilon)=W_{0}(x, t ; \varepsilon)+\varepsilon V_{0}\left(x, \frac{t}{\varepsilon} ; \varepsilon\right)
$$

where $\mathrm{v}_{0}$ is the boundary layer function

$$
v_{0}\left(x, \frac{t}{\varepsilon} ; \varepsilon\right)=-\left\{g(x)+\tilde{f}(x ; \varepsilon)|\tilde{f}(x ; \varepsilon)|^{\nu}\right\} \exp \left(-\frac{t}{\varepsilon}\right)
$$

and $w_{0}$ is the solution of the reduced problem, viz.

$$
W_{0}(x, t ; \varepsilon)=\frac{\tilde{f}(x ; \varepsilon)}{\left\{1+\nu t|\tilde{f}(x ; \varepsilon)|^{\nu}\right\}^{1 / \nu}} .
$$

This function is now twice continuously differentiable with respect to $\mathrm{x}$. Putting 


$$
\mathrm{Z}(\mathrm{x}, t ; \varepsilon)=U(\mathrm{x}, t ; \varepsilon)-\tilde{U}(\mathrm{x}, t ; \varepsilon)+\varepsilon \mathrm{V}_{0}(\mathrm{x}, 0 ; \varepsilon)
$$

we obtain as in section 2 (cf. formula (34))

$$
\left\{\begin{array}{l}
\varepsilon\left\{\frac{\partial^{2} z}{\partial t^{2}}-c^{2}(x, t) \frac{\partial^{2} z}{\partial x^{2}}\right\}+\frac{\partial z}{\partial t}+d^{*}(x, t, z ; \varepsilon)=-R^{*}(x, t ; \varepsilon) \quad(x, t) \in D \\
z(x, 0 ; \varepsilon)=z_{t}(x, 0 ; \varepsilon)=0, \quad-\infty<x<\infty
\end{array}\right.
$$

where

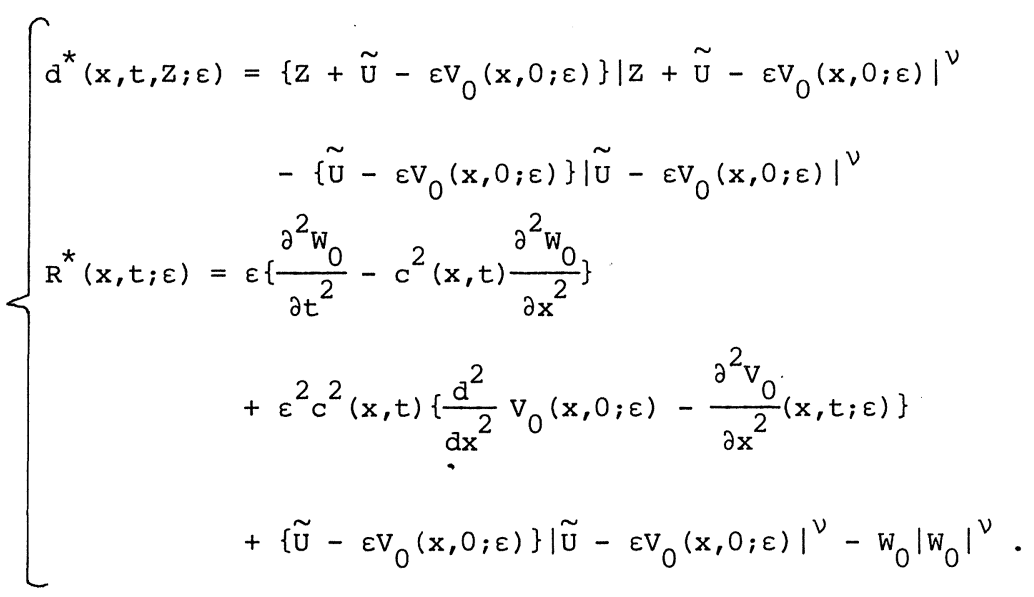

From (67) it follows that

$$
\begin{cases}\tilde{f}^{\prime}(x ; \varepsilon)=0(1), & \varepsilon \downarrow 0 \\ \tilde{f}^{\prime \prime}(x ; \varepsilon)=0\left(\varepsilon^{-p}\right), & \varepsilon \downarrow 0\end{cases}
$$

uniformly valid in each compact subset of $I R$, so using (65) we obtain

$$
\mathrm{R}^{*}(\mathrm{x}, \mathrm{t} ; \varepsilon)=0\left(\varepsilon^{1-\mathrm{p}}\right), \quad \varepsilon \downarrow 0
$$

uniformly valid in each compact subset $\Omega \subset \bar{D}$.

Hence, applying theorem 1 to (73), we obtain

$$
\mathrm{Z}(\mathrm{x}, \mathrm{t} ; \varepsilon)=O\left(\varepsilon^{-\mathrm{p}+\frac{3}{4}}\right), \quad \varepsilon \downarrow 0
$$


uniformly valid in each compact subset $\Omega \subset \bar{D}$, provided that

$$
1-p>\frac{1}{4 \nu}+\frac{1}{4}
$$

Using (70) and (72) we get

$$
U(x, t ; \varepsilon)=W_{0}(x, t ; \varepsilon)+O\left(\varepsilon^{-p+\frac{3}{4}}\right), \quad \varepsilon+0
$$

uniformly valid in each compact subset $\Omega \subset \bar{D}$.

From (61), (69) and (71) we easily derive that

$$
\mathrm{W}_{0}(\mathrm{x}, \mathrm{t} ; \varepsilon)=\mathrm{w}_{0}(\mathrm{x}, \mathrm{t})+0\left(\varepsilon^{\mathrm{p}}\right), \quad \varepsilon \downarrow 0
$$

uniformly valid in each compact subset $\Omega \subset \bar{D}$, whence

$$
\mathrm{U}(\mathrm{x}, \mathrm{t} ; \varepsilon)=\mathrm{w}_{0}(\mathrm{x}, \mathrm{t})+O\left(\varepsilon^{\mathrm{K}}\right), \quad \varepsilon \downarrow 0
$$

uniformly valid in each compact subset $\Omega \subset \overline{\mathrm{D}}$, with

$$
\kappa=\min \left(p,-p+\frac{3}{4}\right) .
$$

In order to estimate the difference between the solution $u$ of the original problem (59) and the solution $U$ of the modified problem (66) we put

$$
z(x, t ; \varepsilon)=u(x, t ; \varepsilon)-\tau(x, t ; \varepsilon)-f(x)+\tilde{f}(x ; \varepsilon) .
$$

Then it follows from (59) and (66) that

$$
\left\{\begin{array}{l}
\varepsilon\left\{\frac{\partial^{2} z}{\partial t^{2}}-c^{2}(x, t) \frac{\partial^{2} z}{\partial x^{2}}\right\}+\frac{\partial z}{\partial t}+\tilde{d}(x, t, z ; \varepsilon)=-\tilde{R}(x, t ; \varepsilon), \quad(x, t) \in D \\
z(x, 0 ; \varepsilon)=z_{t}(x, 0 ; \varepsilon)=0, \quad-\infty<x<\infty
\end{array}\right.
$$

where 
118

$$
\left\{\begin{aligned}
\tilde{d}(x, t, z ; \varepsilon)= & (z+U+f-\tilde{f})|z+U+f-\tilde{f}|^{\nu} \\
& -(U+f-\tilde{f})|U+f-\tilde{f}|^{\nu} \\
\tilde{R}(x, t ; \varepsilon)= & (U+f-\tilde{f})|U+f-\tilde{f}|^{\nu}-U|U|^{\nu} \\
& +\varepsilon c^{2}(x, t)\{\tilde{f} "(x ; \varepsilon)-f "(x)\} .
\end{aligned}\right.
$$

We shall examine the right-hand side function $\widetilde{R}$.

Since the function $w_{0}$ defined by (61) has the same zeroes as $f$ it follows from (68) that

$$
\left|w_{0}(x, t)\right|^{\nu}\{f(x)-\tilde{f}(x ; \varepsilon)\}=0\left(\varepsilon^{p+\nu p}\right), \quad \varepsilon+0
$$

uniformly valid in each compact subset $\Omega \subset \bar{D}$.

Using this result together with the formulae (69) and (75) and the inequality

$$
(x+y)^{\alpha} \leq x^{\alpha}+y^{\alpha} \text { for } x \geq 0, y \geq 0 \text { and } 0<\alpha \leq 1
$$

we obtain for $\varepsilon$ sufficiently small

$$
\begin{aligned}
|(U+f-\tilde{f})| U+f-\left.\tilde{f}\right|^{\nu}-U|U|^{\nu} \mid & =(\nu+1)|f-\tilde{f}| \cdot|U+\theta(f-\tilde{f})|^{\nu} \\
& \leq(\nu+1)|f-\tilde{f}|\left\{|U|^{\nu}+|f-\tilde{f}|^{\nu}\right\} \\
& \leq(\nu+1)|f-\tilde{f}|\left\{\left|w_{0}\right|^{\nu}+|f-\tilde{f}|^{\nu}\right\}+C \varepsilon^{p+\kappa \nu} \\
& \leq C \varepsilon^{p+\kappa \nu}
\end{aligned}
$$

with $0<\theta<1$ and $C$ a generic constant independent of $\varepsilon$.

Hence it follows that

$$
\widetilde{R}(x, t ; \varepsilon)=0\left(\varepsilon^{\lambda}\right), \quad \varepsilon \downarrow 0
$$

uniformly valid in each compact subset $\Omega \subset \bar{D}$, where

$$
\lambda=\min \left\{1-p, p+\nu p, p+\nu\left(\frac{3}{4}-p\right)\right\} .
$$


Applying theorem 1 to (77) we obtain

$$
z(x, t ; \varepsilon)=0\left(\varepsilon^{\lambda-\frac{1}{4}}\right), \quad \varepsilon \downarrow 0
$$

uniformly valid in each compact subset $\Omega \subset \bar{D}$, provided that

$$
\lambda>\frac{1}{4 \nu}+\frac{1}{4} .
$$

Hence, using (69), (75) and (76), we obtain

$$
u(x, t ; \varepsilon)=w_{0}(x, t)+0\left(\varepsilon^{\mu-\frac{1}{4}}\right), \quad \varepsilon+0
$$

uniformly valid in each compact subset $\Omega \subset \bar{D}$, where

$$
\mu=\min \left\{1-p, p+\frac{1}{4}, p+v p, p+v\left(\frac{3}{4}-p\right)\right\}
$$

Next we shall determine the positive constant p. This constant has to be chosen such that the inequalities (74) and (79) are satisfied, where $\lambda$ is given by (78). A straightforward calculation shows that these inequalities can only be fulfilled for some positive value of $p$ if we restrict ourselves to values of $v$ satisfying

$$
\nu>\frac{2}{3}
$$

In that case an optimal value $\mu=\frac{5}{8}$ is obtained if we choose $p=\frac{3}{8}$. So finally we obtain from (80)

$$
u(x, t ; \varepsilon)=w_{0}(x, t)+o\left(\varepsilon^{3 / 8}\right), \quad \varepsilon \downarrow 0
$$

uniformly valid in each compact subset $\Omega \subset \overline{\mathrm{D}}$, provided that $\nu>\frac{2}{3}$.

We summarize the results in

THEOREM 4. Under each of the following restrictions on $\nu$ and $f$ there exists for sufficiently small values of $\varepsilon$ a solution $u$ of problem (59) under the conditions (58), which satisfies

$$
u(x, t ; \varepsilon)=\frac{f(x)}{\left\{1+\nu t|f(x)|^{\nu}\right\}^{1 / \nu}}+0\left(\varepsilon^{\alpha}\right), \quad \varepsilon+0
$$


uniformly in each compact subset $\Omega \subset \overline{\mathrm{D}}$ :

(i) $\quad v=2 \mathrm{k} \quad(\mathrm{k} \in \mathbb{N})$; in this case $\alpha=1$

(ii) $\nu>0$ and $f$ has no zeroes; in this case $\alpha=1$

(iii) $\nu \geq 1$; in this case $\alpha=\frac{3}{4}$

(iv) $\frac{1}{3}<\nu<1$ and zeroes of $f$ have multiplicity $k \geq 2$; in this case $\alpha=\frac{3}{4}$ (v) $\frac{2}{3}<v<1$; in this case $\alpha=\frac{3}{8}$.

Finally we remark that nonlinear initial-boundary value problems in more than one space variable, which are governed by a differential equation of the type discussed in this section, have been studied by GENET \& MADAUNE [17] and MADAUNE [30]. It was shown amongst other things that the solution $\mathrm{u}$ of these kind of problems converges in some integral norm to the solution $\mathrm{w}_{0}$ of the reduced problem as $\varepsilon$ tends to zero.

A discussion of nonlinearities of the type $u|u|^{\nu}$ is also given by LIONS [29]. 


\section{CHAPTER V}

INITIAL - BOUNDARY VALUE PROBLEMS FOR LINEAR HYPERBOLIC DIFFERENTIAL EQUATIONS

In this chapter we shall discuss the initial-boundary value problem (singular perturbation problem)

(1) $\begin{cases}\varepsilon L_{2}[u]+L_{1}^{(\varepsilon)}[u]=f(x, t ; \varepsilon), \quad & 0 \leq x \leq l \\ u(x, 0 ; \varepsilon)=g(x ; \varepsilon), & 0 \leq x \leq l \\ u_{t}(x, 0 ; \varepsilon)=h(x ; \varepsilon), & 0 \leq t<\infty \\ u(0, t ; \varepsilon)=k_{1}(t ; \varepsilon), & 0 \leq t<\infty \\ u(l, t ; \varepsilon)=k_{2}(t ; \varepsilon), & 0 .\end{cases}$

where $\varepsilon$ is a small positive parameter, i.e. $0<\varepsilon<<1$, the region $D$ is the strip

$$
D=\{(x, t) \mid 0<x<\ell, 0<t<\infty\}
$$

and the differential operators $L_{2}$ and $L_{1}^{(\varepsilon)}$ are defined by

$$
\left\{\begin{array}{l}
L_{2}[u]=\frac{\partial^{2} u}{\partial t^{2}}-c^{2}(x, t) \frac{\partial^{2} u}{\partial x^{2}} \\
L_{1}^{(\varepsilon)}[u]=a(x, t ; \varepsilon) \frac{\partial u}{\partial t}+b(x, t ; \varepsilon) \frac{\partial u}{\partial x}+d(x, t ; \varepsilon) u
\end{array}\right.
$$

The functions $a, b, d, f, g, h, k_{1}$ and $k_{2}$ depend on the variables $x$ and/or $t$ and on the parameter $\varepsilon ;$ the function $c$ satisfies

$$
c(x, t)>0 \quad \text { in } \bar{D} \text {, }
$$

which implies that $\mathrm{L}_{2}$ is a hyperbolic operator. 
It will be assumed that the boundary functions $g, k_{1}$ and $k_{2}$ are continuous, no exception being made for the corners of the strip; so we require in particular

$$
\left\{\begin{array}{l}
g(0 ; \varepsilon)=k_{1}(0 ; \varepsilon) \\
g(l ; \varepsilon)=k_{2}(0 ; \varepsilon)
\end{array}\right.
$$

valid for all $\varepsilon$ with $0<\varepsilon \ll 1$.

Furthermore we shall impose the growth conditions discussed in section 1 of chapter III (cf. formula (7) of that chapter), that is, we assume the coefficient $a(x, t ; \varepsilon)$ to be strictly positive and we require that the functions $a, b$ and $c$ are such that for sufficiently small values of $\varepsilon$ the subcharacteristics of $L_{1}^{(\varepsilon)}$ are timelike with respect to the characteristic directions connected with $\mathrm{L}_{2}$.

There exists few literature on singularly perturbed initial-boundary value problems for hyperbolic differential equations. For problems in $\mathrm{IR}^{2}$ with constant coefficients an analysis based on Laplace transforms is given in WHITHAM [56, chapter 10]. Initial-boundary value problems with more than one space variable have been investigated by DZAVADOV [8] and GENET \& PUPION [18] (linear problems) and by GENET \& MADAUNE [17] and MADAUNE [30] (nonlinear problems).

Using a modified version of the energy integral method developed in chapter III we shall derive in section 1 a priori estimates for the solution $\mathrm{u}$ of problem (1). In section 2 we shall construct an approximation for the solution $u$ of a problem of the type (1), in which the parameter $\varepsilon$ occurs only as a factor in front of the operator $\mathrm{L}_{2}$.

REMARK. It is possible to consider problem (1) with a more general hyperbolic operator $\mathrm{L}_{2}$ defined by

$$
L_{2}[u]=\frac{\partial^{2} u}{\partial t^{2}}+\left\{c_{1}(x, t)+c_{2}(x, t)\right\} \frac{\partial^{2} u}{\partial x \partial t}+c_{1}(x, t) c_{2}(x, t) \frac{\partial^{2} u}{\partial x^{2}}
$$

with $c_{1}(x, t)<0<c_{2}(x, t)$ in $\bar{D}$. However, since all essential features of the general theory become manifest in the special case where 


$$
-c_{1}(x, t) \equiv c_{2}(x, t) \equiv c(x, t)>0 \quad \text { in } \bar{D}
$$

we prefer to discuss the latter for reasons of simplicity.

\section{A priori estimates}

We consider the initial-boundary value problem (1) under the following conditions

(2)

$$
\left\{\begin{array}{l}
c(x, t) \in C^{1}(\bar{D}) \\
c(x, t)>0 \text { in } \bar{D}
\end{array}\right.
$$

for each compact subset $\Omega \subset \bar{D}$ there exists a positive constant $\varepsilon_{1}$ (depending on $\Omega$ ) such that

(i) $\quad a(x, t ; \varepsilon) \in C^{1}(\Omega), \quad b(x, t ; \varepsilon) \in C^{1}(\Omega)$

$$
\mathrm{d}(\mathrm{x}, \mathrm{t} ; \varepsilon) \in \mathrm{C}^{0}(\Omega), \quad f(x, t ; \varepsilon) \in \mathrm{C}^{0}(\Omega)
$$

valid for every value of $\varepsilon$ satisfying $0<\varepsilon \leq \varepsilon_{1}$

(ii) the functions $a(x, t ; \varepsilon), b(x, t ; \varepsilon)$ and their first derivatives, as well as the function $d(x, t ; \varepsilon)$, are uniformly bounded in $\Omega$ for

(3) $0<\varepsilon \leq \varepsilon_{1}$

(iii) $a(x, t ; \varepsilon) \geq a_{0}>0$

valid for all $(x, t) \in \Omega$ and all $\varepsilon \in\left(0, \varepsilon_{1}\right]$, where $a_{0}$ is some constant depending on $\Omega$ and $\varepsilon_{1}$ but independent of $\varepsilon$

(iv) $\left\{\begin{array}{l}a(x, t ; \varepsilon) c(x, t)+b(x, t ; \varepsilon) \geq p>0 \\ a(x, t ; \varepsilon) c(x, t)-b(x, t ; \varepsilon) \geq p>0\end{array}\right.$

valid for all $(x, t) \in \Omega$ and all $\varepsilon \in\left(0, \varepsilon_{1}\right]$, where $p$ is some constant depending on $\Omega$ and $\varepsilon_{1}$ but independent of $\varepsilon$ 
for each positive constant $T$ there exists a positive constant $\varepsilon_{2}$ (depending on $T$ ) such that

$$
\begin{aligned}
& g(x ; \varepsilon) \in C^{1}[0, \ell], \quad h(x ; \varepsilon) \in C^{0}[0, \ell] \\
& k_{1}(t ; \varepsilon) \in C^{1}[0, T], \quad k_{2}(t ; \varepsilon) \in C^{1}[0, T]
\end{aligned}
$$

(4) $\{$ and

$$
\left\{\begin{array}{l}
g(0 ; \varepsilon)=\mathrm{k}_{1}(0 ; \varepsilon) \\
g(\ell ; \varepsilon)=\mathrm{k}_{2}(0 ; \varepsilon)
\end{array}\right.
$$

valid for every value of $\varepsilon$ satisfying $0<\varepsilon \leq \varepsilon_{2}$.

In order to derive a priori estimates for the solution $u(x, t ; \varepsilon)$ of (1) in some compact subset $S \subset \bar{D}$ we enclose $S$ by a rectangle

$$
\Omega=\{(x, t) \mid 0 \leq x \leq \ell, 0 \leq t \leq T\}
$$

where $T \geq \sup _{(x, t) \in S} t$ (see figure 1$)$.

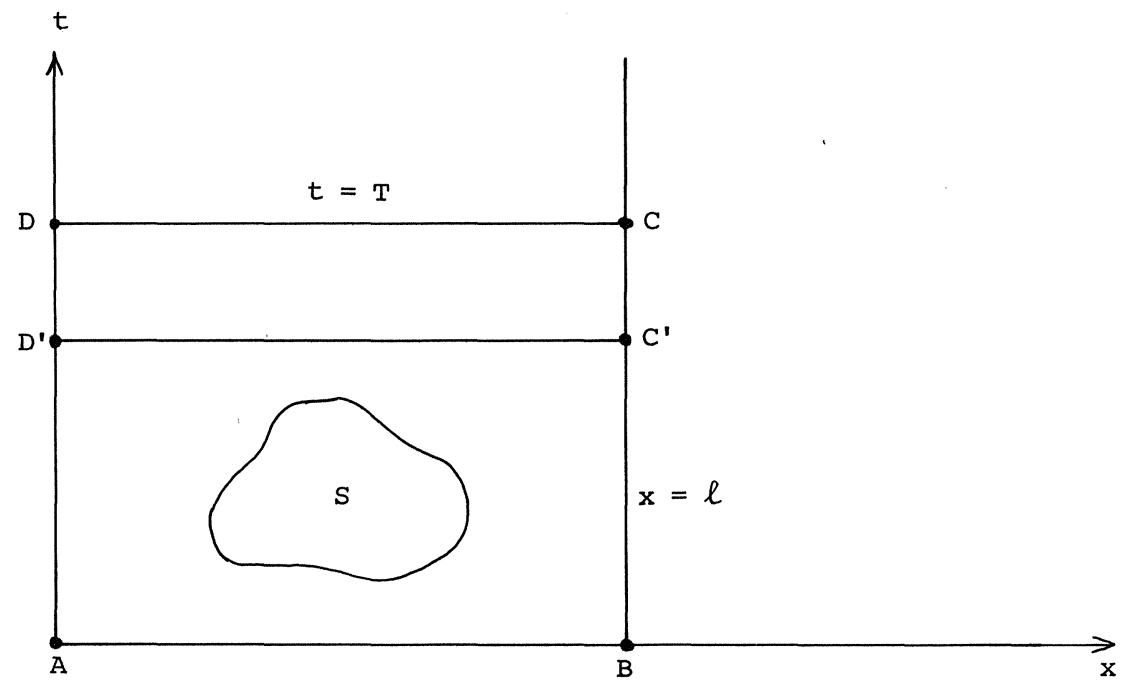

Figure 1 
Let $\alpha$ be some positive constant (independent of $\varepsilon$ ) to be determined later on and let $\beta=\beta(x, t ; \varepsilon)$ and $\gamma=\gamma(x, t ; \varepsilon)$ be continuously differentiable functions (depending on the variables $x$ and $t$ and on the parameter $\varepsilon$ ) likewise to be specified later on.

We multiply the differential equation (1) by 2 aau to obtain

$$
\begin{aligned}
& \frac{\partial}{\partial t}\left\{\alpha a^{2} u^{2}+2 \varepsilon \alpha a u u_{t}\right\}+\frac{\partial}{\partial x}\left\{\alpha a b u^{2}-2 \varepsilon \alpha a c^{2} u u_{x}\right\}= \\
& u^{2}\left\{-2 \alpha a d+\frac{\partial\left(\alpha a^{2}\right)}{\partial t}+\frac{\partial(\alpha a b)}{\partial x}\right\}+2 \varepsilon u u_{t} \frac{\partial(\alpha a)}{\partial t}-2 \varepsilon u u_{x} \frac{\partial\left(\alpha a c^{2}\right)}{\partial x} \\
& +2 \varepsilon \alpha a u_{t}^{2}-2 \varepsilon \alpha a c^{2} u_{x}^{2}+2 \alpha a f u
\end{aligned}
$$

valid for $(x, t) \in \Omega$ and $0<\varepsilon \leq \varepsilon_{0}=\min \left(\varepsilon_{1}, \varepsilon_{2}\right)$, the values of $\varepsilon_{1}$ and $\varepsilon_{2}$ being determined by the region $\Omega$ (see the conditions $(3, i)$ and $(4)$ ).

Multiplication of (1) by $2 \beta u_{t}$ yields

(6)

$$
\begin{aligned}
& \frac{\partial}{\partial t}\left\{\varepsilon \beta u_{t}^{2}+\varepsilon \beta c^{2} u_{x}^{2}\right\}-\frac{\partial}{\partial x}\left\{2 \varepsilon \beta c^{2} u_{t} u_{x}\right\}= \\
& \varepsilon u_{t}^{2} \frac{\partial \beta}{\partial t}-2 \varepsilon u_{t} u_{x} \frac{\partial\left(\beta c^{2}\right)}{\partial x}+\varepsilon u_{x}^{2} \frac{\partial\left(\beta c^{2}\right)}{\partial t} \\
& -2 \beta d u u_{t}-2 \beta a u_{t}^{2}-2 \beta b u_{t} u_{x}+2 \beta f u_{t}
\end{aligned}
$$

valid for $(x, t) \in \Omega$ and $0<\varepsilon \leq \varepsilon_{0}$.

Finally, multiplication of (1) by $2 \gamma u_{x}$ leads to

$$
\begin{aligned}
& \frac{\partial}{\partial t}\left\{2 \varepsilon \gamma u_{t} u_{x}\right\}-\frac{\partial}{\partial x}\left\{\varepsilon \gamma u_{t}^{2}+\varepsilon \gamma c^{2} u_{x}^{2}\right\}= \\
& -\varepsilon u_{t}^{2} \frac{\partial \gamma}{\partial x}+2 \varepsilon u_{t} u_{x} \frac{\partial \gamma}{\partial t}-\varepsilon u_{x}^{2} \frac{\partial\left(\gamma c^{2}\right)}{\partial x} \\
& -2 \gamma d u_{x}-2 \gamma a u_{t} u_{x}-2 \gamma b u_{x}^{2}+2 \gamma f u_{x}
\end{aligned}
$$

likewise valid for $(x, t) \in \Omega$ and $0<\varepsilon \leq \varepsilon_{0}$. 
Adding (5), (6) and (7) we obtain

$$
\frac{\partial}{\partial t} Q_{1}+\frac{\partial}{\partial x} Q_{2}=Q_{3}+Q_{4}
$$

where the expressions $Q_{i}(i=1,2,3,4)$ are given by

(9)

$$
\left\{\begin{aligned}
Q_{1}= & \alpha a^{2} u^{2}+2 \varepsilon \alpha a u_{t}+\varepsilon \beta u_{t}^{2}+2 \varepsilon \gamma u_{t} u_{x}+\varepsilon \beta c^{2} u_{x}^{2} \\
Q_{2}= & -\varepsilon \gamma c^{2} u_{x}^{2}-2 \varepsilon \alpha a c^{2} u_{x}-2 \varepsilon \beta c^{2} u_{t} u_{x}+\alpha a b u^{2}-\varepsilon \gamma u_{t}^{2} \\
Q_{3}= & u^{2}\left\{-2 \alpha a d+\frac{\partial\left(\alpha a^{2}\right)}{\partial t}+\frac{\partial(\alpha a b)}{\partial x}\right\}+2 \varepsilon u u_{t} \frac{\partial(\alpha a)}{\partial t}-2 \varepsilon u u_{x} \frac{\partial\left(\alpha a c^{2}\right)}{\partial x} \\
& +\varepsilon u_{t}^{2}\left\{\frac{\partial \beta}{\partial t}-\frac{\partial \gamma}{\partial x}\right\}+2 \varepsilon u_{t} u_{x}\left\{\frac{\partial \gamma}{\partial t}-\frac{\partial\left(\beta c^{2}\right)}{\partial x}\right\}+\varepsilon u_{x}^{2}\left\{\frac{\partial\left(\beta c^{2}\right)}{\partial t}-\frac{\partial\left(\gamma c^{2}\right)}{\partial x}\right\} \\
Q_{4}= & -2 \beta d u u_{t}-2 \gamma d u u_{x}+2 \alpha a f u+2 \beta f u_{t}+2 \gamma f u_{x} \\
& -2 a(\beta-\varepsilon \alpha) u_{t}^{2}-2(\gamma a+\beta b) u_{t} u_{x}-2\left(\gamma b+\varepsilon \alpha a c^{2}\right) u_{x}^{2} .
\end{aligned}\right.
$$

Integrating (8) over the rectangle $\Omega$ and using GREEN's theorem in the plane we obtain (see figure 1)

$$
\int_{D}^{C} Q_{1} d x=\int_{A}^{B} Q_{1} d x+\int_{A}^{D} \Omega_{2} d t-\int_{B}^{C} \Omega_{2} d t+\iint_{\Omega}\left(Q_{3}+Q_{4}\right) d x d t .
$$

We shall now specify the functions $\beta$ and $\gamma$.

Let $\delta$ be an arbitrarily small positive number (independent of $\varepsilon$ ) satisfying $0<\delta<\frac{1}{2} l$. Let $\Psi(\mathrm{x})$ be some $C^{\infty}$-function defined for $0 \leq \mathrm{x} \leq \ell$ which satisfies

$$
\left\{\begin{array}{l}
\Psi(x)= \begin{cases}1 & \text { for } \delta \leq x \leq \frac{1}{2} l \\
0 & \text { for } 0 \leq x \leq \frac{1}{2} \delta\end{cases} \\
\Psi(x) \text { monotonically increasing for } \frac{1}{2} \delta \leq x \leq \delta \\
\Psi(x)=\Psi(\ell-x) \quad \text { for } \frac{1}{2} l \leq x \leq \ell
\end{array}\right.
$$


(see figure $2 \mathrm{a}$ ) and let the $c^{\infty}$-function $\Phi(\mathrm{x} ; \varepsilon)$ by defined by

$$
\Phi(x ; \varepsilon)=\varepsilon+(1-\varepsilon) \Psi(x) \text { for } 0 \leq x \leq \ell
$$

(see figure $2 \mathrm{~b}$ ).

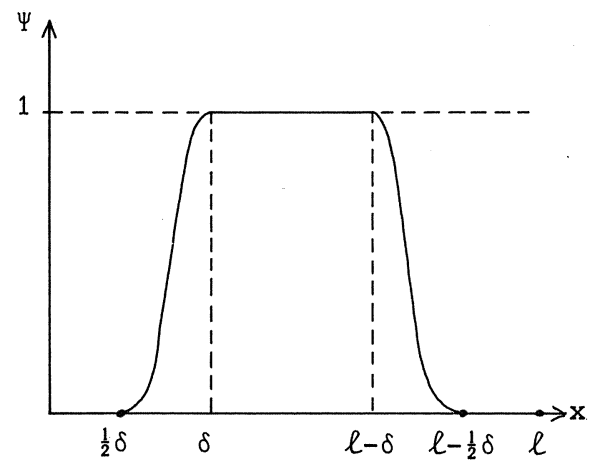

Figure 2a

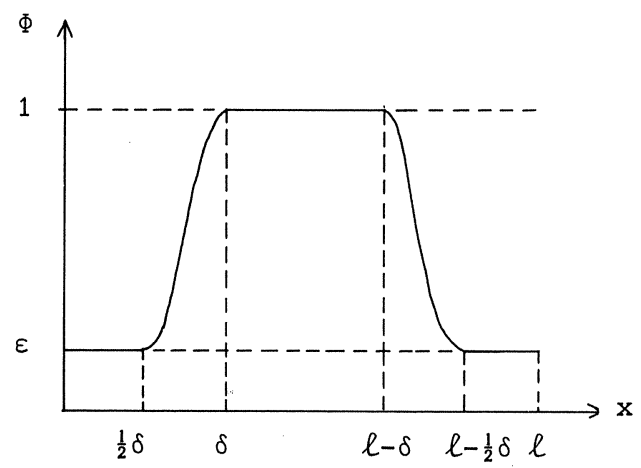

Figure 2b

We now choose the functions $\beta$ and $\gamma$ as follows

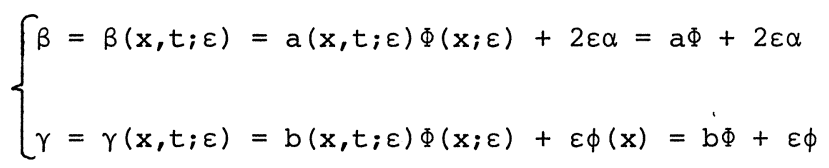

where $\phi(x)$ is some $C^{\infty}$-function defined for $0 \leq x \leq \ell$, which will be specified later on. This leaves the constant $\alpha$ and the function $\phi$ to be determined.

Now that we have chosen $\beta$ and $\gamma$ we shall have a closer look at the expressions $Q_{i}(i=1,2,3,4)$. Let us start with $Q_{2}$. The integrals along $A D$ and $B C$ in the right-hand side of (10) contain in their integrands the function $u_{x}$ that we do not know a priori along $A D$ and $B C$. This difficulty can be overcome by choosing the function $\phi$ such that $\gamma \geq \varepsilon$ along $A D$ and $\gamma \leq-\varepsilon$ along $B C$. Since we have from (11), (12) and (13) 
128

$$
\begin{cases}\gamma(0, t ; \varepsilon)=\varepsilon b(0, t ; \varepsilon)+\varepsilon \phi(0) & \text { along } A D \\ \gamma(l, t ; \varepsilon)=\varepsilon b(l, t ; \varepsilon)+\varepsilon \phi(l) & \text { along } B C\end{cases}
$$

this can be achieved by choosing $\phi$ such that

$$
\left\{\begin{array}{l}
\phi(0) \geq B+1 \\
\phi(\ell) \leq-B-1
\end{array}\right.
$$

where the $\varepsilon$-independent nonnegative constant $B$ is defined by

$$
\begin{aligned}
B= & \sup _{(x, t) \in \Omega}|b(x, t ; \varepsilon)| \\
& 0<\varepsilon \leq \varepsilon_{0}
\end{aligned}
$$

(cf. the condition $(3, i i))$. A possible choice for $\phi$ is given by

$$
\phi(x)=(B+1)\left(1-\frac{2 x}{\ell}\right) \quad \text { for } 0 \leq x \leq \ell
$$

With this choice we obtain along $A D$, using (9), (13) and the inequality $\gamma(0, t ; \varepsilon) \geq \varepsilon$

$$
\begin{aligned}
Q_{2} & \leq-\varepsilon^{2} c^{2} u_{x}^{2}-2 \varepsilon \alpha a c^{2} u_{x}-2 \varepsilon^{2}(a+2 \alpha) c^{2} u_{t} u_{x}+\alpha a b u^{2}-\varepsilon^{2} u_{t}^{2} \\
& =-2 c^{2}\left(\frac{1}{2} \varepsilon u_{x}+\alpha a u\right)^{2}-2 \varepsilon^{2} c^{2}\left\{\frac{1}{2} u_{x}+(a+2 \alpha) u_{t}\right\}^{2}
\end{aligned}
$$

$$
\begin{aligned}
& +\left(\alpha a b+2 \alpha^{2} a^{2} c^{2}\right) u^{2}+\varepsilon^{2}\left\{2(a+2 \alpha)^{2} c^{2}-1\right\} u_{t}^{2} \\
\leq & M_{1}\left(u^{2}+\varepsilon^{2} u_{t}^{2}\right)
\end{aligned}
$$

and similarly along $B C$, using (9), (13) and the inequality $\gamma(\ell, t ; \varepsilon) \leq-\varepsilon$

$$
\begin{aligned}
Q_{2} \geq & \varepsilon^{2} c^{2} u_{x}^{2}-2 \varepsilon \alpha a c^{2} u_{x}-2 \varepsilon^{2}(a+2 \alpha) c^{2} u_{t} u_{x}+\alpha a b u^{2}+\varepsilon^{2} u_{t}^{2} \\
= & 2 c^{2}\left(\frac{1}{2} \varepsilon u_{x}-\alpha a u\right)^{2}+2 \varepsilon^{2} c^{2}\left\{\frac{1}{2} u_{x}-(a+2 \alpha) u_{t}\right\}^{2} \\
& +\left(\alpha a b-2 \alpha^{2} a^{2} c^{2}\right) u^{2}+\varepsilon^{2}\left\{1-2(a+2 \alpha)^{2} c^{2}\right\} u_{t}^{2} \geq
\end{aligned}
$$




$$
\geq-M_{2}\left(u^{2}+\varepsilon^{2} u_{t}^{2}\right)
$$

for certain positive constants $M_{1}$ and $M_{2}$ depending on $\Omega$ but independent of $\varepsilon$. As for the expression $Q_{3}$, it is easily established that there exist positive constants $M_{3}$ (depending on $\Omega$ and $\alpha$ but independent of $\varepsilon$ ) and $M_{4}$ (depending on $\Omega$ but independent of $\alpha$ and $\varepsilon$ ) such that

$$
Q_{3} \leq M_{3}\left(u^{2}+\varepsilon^{2} u_{t}^{2}+\varepsilon^{2} u_{x}^{2}\right)+\varepsilon M_{4}\left(u_{t}^{2}+u_{x}^{2}\right)
$$

valid for $(x, t) \in \Omega$ and $0<\varepsilon \leq \varepsilon_{0}$. The independence of $M_{4}$ with respect to $\alpha$ follows from the observation that the terms $a \Phi$ and $b \Phi$ in (13) are independent of $\alpha$.

Next we consider $Q_{4}$. Substituting (13) into (9) we obtain after some calculations

$$
\begin{aligned}
Q_{4}= & -2 \Phi\left\{\left(a u_{t}+b u_{x}\right)^{2}+(d u-f)\left(a u_{t}+b u_{x}\right)\right\} \\
& -4 \varepsilon \alpha d u u_{t}-2 \varepsilon \phi d u u_{x}+2 \alpha a f u+4 \varepsilon \alpha f u_{t}+2 \varepsilon \phi f u_{x} \\
& -2 \varepsilon \alpha\left\{a u_{t}^{2}+\left(2 b+\frac{a \phi}{\alpha}\right) u_{t} u_{x}+\left(a c^{2}+\frac{b \phi}{\alpha}\right) u_{x}^{2}\right\} .
\end{aligned}
$$

Using $(3, i v)$ it is not difficult to verify that there exists a positive constant $\mathrm{p}_{0}$, independent of $\alpha$ and $\varepsilon$ and satisfying

$$
\begin{gathered}
0<\mathrm{p}_{0}<1-\sup _{\substack{(x, t) \in \Omega \\
0}} \frac{|b|}{a c}, \\
0<\varepsilon \leq \varepsilon_{0}
\end{gathered}
$$

such that for sufficiently large $\alpha$, say $\alpha>\alpha_{1}$

$$
a u_{t}^{2}+\left(2 b+\frac{a \phi}{\alpha}\right) u_{t} u_{x}+\left(a c^{2}+\frac{b \phi}{\alpha}\right) u_{x}^{2} \geq p_{0}\left(a u_{t}^{2}+a c^{2} u_{x}^{2}\right)
$$

valid for $(x, t) \in \Omega$ and $0<\varepsilon \leq \varepsilon_{0}$.

Hence it follows that there exist for $\alpha>\alpha_{1}$ positive constants $M_{5}$ (depending on $\Omega$ and $\alpha$ but independent of $\varepsilon$ ) and $M_{6}$ (depending on $\Omega$ but independent of $\alpha$ and $\varepsilon$ ) such that 


$$
Q_{4} \leq M_{5}\left(u^{2}+\varepsilon^{2} u_{t}^{2}+\varepsilon^{2} u_{x}^{2}+f^{2}\right)-\varepsilon \alpha M_{6}\left(u_{t}^{2}+u_{x}^{2}\right)
$$

valid for $(x, t) \in \Omega$ and $0<\varepsilon \leq \varepsilon_{0}$.

Taking $\alpha$ such that

$$
\alpha>\alpha_{2}=\max \left(\alpha_{1}, \frac{M_{4}}{M_{6}}\right)
$$

we obtain from (16) and (17), using (12)

$$
Q_{3}+Q_{4} \leq\left(M_{3}+M_{5}\right)\left(u^{2}+\varepsilon^{2} u_{t}^{2}+\varepsilon^{2} u_{x}^{2}+f^{2}\right)
$$

$$
\leq\left(M_{3}+M_{5}\right)\left(u^{2}+\varepsilon \Phi u_{t}^{2}+\varepsilon \Phi u_{x}^{2}+f^{2}\right)
$$

valid for $(x, t) \in \Omega$ and $0<\varepsilon \leq \varepsilon_{0}$.

Finally we investigate the expression $Q_{1}$. Substituting (13) into (9) we obtain

$$
\begin{aligned}
Q_{1}= & \alpha a^{2} u^{2}+2 \varepsilon \alpha a u_{t}+2 \varepsilon^{2} \alpha u_{t}^{2}+2 \varepsilon^{2} \phi u_{t} u_{x}+2 \varepsilon^{2} \alpha c^{2} u_{x}^{2} \\
& +\varepsilon a \Phi u_{t}^{2}+2 \varepsilon b \Phi u_{t} u_{x}+\varepsilon a c^{2} \Phi u_{x}^{2} \\
= & \frac{1}{4} \alpha a^{2} u^{2}+\frac{3}{4} \alpha\left(a u+\frac{4}{3} \varepsilon u_{t}\right)^{2}+2 \varepsilon^{2}\left(\frac{1}{3} \alpha u_{t}^{2}+\phi u_{t} u_{x}+\alpha c^{2} u_{x}^{2}\right) \\
& +\varepsilon \Phi\left(a u_{t}^{2}+2 b u_{t} u_{x}+a c^{2} u_{x}^{2}\right) .
\end{aligned}
$$

It follows from the conditions $(3, i i i)$ and $(3, i v)$ that there exists a positive constant $\mathrm{m}^{*}$, depending on $\Omega$ but independent of $\varepsilon$, such that

$$
a u_{t}^{2}+2 b u_{t} u_{x}+a c^{2} u_{x}^{2} \geq m^{*}\left(u_{t}^{2}+u_{x}^{2}\right)
$$

valid for $(x, t) \in \Omega$ and $0<\varepsilon \leq \varepsilon_{0}$. Moreover, the expression

$$
\frac{1}{3} \alpha u_{t}^{2}+\phi u_{t} u_{x}+\alpha c^{2} u_{x}^{2}
$$

is definite positive if we choose $\alpha$ sufficiently large, say $\alpha>\alpha_{3}$. So taking for the constant $\alpha$ 


$$
\alpha=\alpha_{0}=\max \left(\alpha_{2}, \alpha_{3}\right)
$$

and using the condition $(3, i i i)$ we obtain the inequality

$$
Q_{1} \geq m\left(u^{2}+\varepsilon \Phi u_{t}^{2}+\varepsilon \Phi u_{x}^{2}\right)
$$

valid for $(x, t) \in \Omega$ and $0<\varepsilon \leq \varepsilon_{0}$, where $m$ is some positive constant depending on $\Omega$ but independent of $\varepsilon$.

It is also easily established that

$$
Q_{1} \leq M_{7}\left(u^{2}+\varepsilon u_{t}^{2}+\varepsilon u_{x}^{2}\right)
$$

valid for $(x, t) \in \Omega$ and $0<\varepsilon \leq \varepsilon_{0}$ with $M_{7}$ some positive constant depending on $\Omega$ but independent of $\varepsilon$.

Putting

$$
M=\max \left(M_{1}, M_{2}, M_{3}+M_{5}, M_{7}\right)
$$

we obtain from (10), (14), (15), (18), (19) and (20)

$$
m \int_{D}^{C}\left(u^{2}+\varepsilon \Phi u_{t}^{2}+\varepsilon \Phi u_{x}^{2}\right) d x \leq M \iint_{A B C D}\left(u^{2}+\varepsilon \Phi u_{t}^{2}+\varepsilon \Phi u_{x}^{2}\right) d x d t+M K^{2}(\Omega, \varepsilon)
$$

valid for $0<\varepsilon \leq \varepsilon_{0}$ where the positive constant $\mathrm{K}(\Omega, \varepsilon)$, depending on $\Omega$ and $\varepsilon$, is defined by

$$
\begin{aligned}
\mathrm{K}(\Omega, \varepsilon)= & \|f\|_{\Omega}+\|\mathrm{g}\|_{[0, \ell]}+\left\|\mathrm{k}_{1}\right\|_{[0, T]}+\left\|\mathrm{k}_{2}\right\|_{[0, T]} \\
& +\varepsilon \varepsilon^{\frac{1}{2}}\left\|_{\mathrm{X}}\right\|_{[0, \ell]}+\varepsilon\left\|^{\frac{1}{2}}\right\|_{[0, \ell]}+\varepsilon\left\|_{\mathrm{k}_{1}}\right\|_{[0, T]}+\varepsilon\left\|_{\mathrm{k}_{2}}\right\|_{[0, T]}
\end{aligned}
$$

with $\|\cdot\|_{\Omega}$ denoting the $L_{2}$-norm over $\Omega$, etc.

Since (21) with the same values of $\mathrm{m}$ and $\mathrm{M}$ is also valid for every rectangle $\Omega^{\prime}=A A^{\prime} D^{\prime}$ inside $\Omega=A B C D$ (see figure 1 ) we obtain as in section 2 of chapter III, using GRONWALL's lemma, the estimate

$$
\int_{0}^{\ell}\left(u^{2}+\varepsilon \Phi u_{t}^{2}+\varepsilon \Phi u_{x}^{2}\right) d x \leq C(\Omega) k^{2}(\Omega, \varepsilon)
$$


(cf. formula (26) of chapter III), whence particularly

$$
\int_{0}^{\ell}\left(u^{2}+\varepsilon^{2} u_{t}^{2}+\varepsilon^{2} u_{x}^{2}\right) d x \leq C(\Omega) k^{2}(\Omega, \varepsilon)
$$

and

$$
\int_{\delta}^{\ell-\delta}\left(u^{2}+\varepsilon u_{t}^{2}+\varepsilon u_{x}^{2}\right) d x \leq C(\Omega) K^{2}(\Omega, \varepsilon)
$$

valid for $0 \leq t \leq T$ and $0<\varepsilon \leq \varepsilon_{0}$; the constant $C(\Omega)$, depending on $\Omega$ but independent of $\varepsilon$, is given by

$$
C(\Omega)=\frac{M}{m} \exp \left(\frac{M T}{m}\right)
$$

Pointwise estimates for $u(x, t ; \varepsilon)$ can be obtained from (22) and (23) by applying SOBOLEV's inequality (cf. AGMON [1, p.32]; see also formula (33a) of chapter III). We formulate the results in

THEOREM 1. Let $\mathrm{u}(\mathrm{x}, \mathrm{t} ; \varepsilon)$ be the solution of the initial-boundary value problem (1) under the conditions (2), (3) and (4), and let $\Omega$ be the closed rectangle $0 \leq \mathrm{x} \leq \ell, 0 \leq \mathrm{t} \leq \mathrm{T}$. Then we have for $\varepsilon$ sufficiently small, say $0<\varepsilon \leq \varepsilon_{0}$ with $\varepsilon_{0}$ depending on $\Omega$, the following estimates in $\mathrm{L}_{2}$-norm $\left\{\begin{array}{l}\int_{0}^{l}\left(u^{2}+\varepsilon^{2} u_{t}^{2}+\varepsilon^{2} u_{x}^{2}\right) d x \leq c(\Omega) k^{2}(\Omega, \varepsilon) \quad \text { valid for } 0 \leq t \leq T \\ \int_{\delta}^{l-\delta}\left(u^{2}+\varepsilon u_{t}^{2}+\varepsilon u_{x}^{2}\right) d x \leq c(\Omega) k^{2}(\Omega, \varepsilon) \quad \text { valid for } 0 \leq t \leq T .\end{array}\right.$

We have also the following pointwise estimates

(24a) $\quad\left\{\begin{array}{l}\sup _{\Omega}|u(x, t ; \varepsilon)| \leq C(\Omega) \varepsilon^{-\frac{1}{2}} K(\Omega, \varepsilon) \\ \sup _{\delta \leq x \leq \ell-\delta}|u(x, t ; \varepsilon)| \leq C(\Omega) \varepsilon^{-\frac{1}{4}} K(\Omega, \varepsilon) . \\ 0 \leq t \leq T\end{array}\right.$

Here $\mathrm{C}(\Omega)$ is some positive constant depending on $\Omega$ but independent of $\varepsilon, \delta$ is an arbitrarily small positive constant independent of $\varepsilon$ and satisfying 
$0<\delta<\frac{1}{2} \ell$.

The positive constant $\mathrm{K}(\Omega, \varepsilon)$, depending on both $\Omega$ and $\varepsilon$, is given by

$$
\begin{aligned}
\mathrm{K}(\Omega, \varepsilon)= & \|f\|_{\Omega}+\|\mathrm{g}\|_{[0, \ell]}+\left\|\mathrm{k}_{1}\right\|_{[0, \mathrm{~T}]}+\left\|\mathrm{k}_{2}\right\|_{[0, \mathrm{~T}]} \\
& +\varepsilon^{\frac{1}{2}}\left\|_{\mathrm{X}}\right\|_{[0, \ell]}+\varepsilon^{\frac{1}{2} \|}\left\|_{[0, \ell]}+\varepsilon\right\|_{\mathrm{k}_{1}}\left\|_{[0, \mathrm{~T}]}+\varepsilon\right\|_{\mathrm{k}_{2}} \|[0, \mathrm{~T}]
\end{aligned}
$$

with $\|\cdot\|_{\Omega}$ denoting the $\mathrm{L}_{2}$-norm over $\Omega$, etc.

\section{REMARKS.}

1. It is possible to obtain pointwise estimates for the derivatives $u_{x}$ and $u_{t}$ by taking into account integrals along characteristics (cf. chapter III, section 2). It follows after tedious calculations that

$$
\left\{\begin{array}{l}
\sup _{\Omega}\left|\frac{\partial u}{\partial x}(x, t ; \varepsilon)\right| \leq C(\Omega) \varepsilon^{-2} \mathrm{~K}_{1}(\Omega, \varepsilon) \\
\sup _{\Omega}\left|\frac{\partial u}{\partial t}(x, t ; \varepsilon)\right| \leq C(\Omega) \varepsilon^{-2} \mathrm{~K}_{1}(\Omega, \varepsilon),
\end{array}\right.
$$

the positive constant $\mathrm{K}_{1}(\Omega, \varepsilon)$ being given by

$$
\begin{aligned}
\mathrm{K}_{1}(\Omega, \varepsilon)= & \sup _{\Omega}|\mathrm{f}|+\|\mathrm{g}\|_{[0, \ell]}+\left\|\mathrm{k}_{1}\right\|_{[0, \mathrm{~T}]}+\left\|\mathrm{k}_{2}\right\|_{[0, \mathrm{~T}]} \\
& +\varepsilon \sup _{[0, \ell]}\left|g_{\mathrm{x}}\right|+\varepsilon \sup _{[0, \ell]}|\mathrm{h}|+\varepsilon \sup _{[0, \mathrm{~T}]}\left|\mathrm{k}_{1}\right|+\varepsilon \sup _{t}\left|\mathrm{k}_{2}\right|
\end{aligned}
$$

(cf. the formulae (33b), (33c) of chapter III).

2. Other estimates are possible if it is given that

$$
b(0, t ; \varepsilon) \geq b_{0}>0 \text { for } 0 \leq t \leq T \text { and } 0<\varepsilon \leq \varepsilon_{0}
$$

where $b_{0}$ is some constant depending on $T$ and $\varepsilon_{0}$ but independent of $\varepsilon$; this condition implies the absence of a boundary layer at $x=0, t \geq 0$. Replacing (11) by

$$
\left\{\begin{array}{l}
\Psi(x)= \begin{cases}1 & \text { for } 0 \leq x \leq \ell-\delta \\
0 & \text { for } l-\frac{1}{2} \delta \leq x \leq l\end{cases} \\
\Psi(x) \text { monotonically decreasing for } l-\delta \leq x \leq \ell-\frac{1}{2} \delta
\end{array}\right.
$$


and adjusting the proof of theorem 1 we obtain

$$
\left\{\begin{array}{l}
\int_{0}^{\ell-\delta}\left(u^{2}+\varepsilon u_{t}^{2}+\varepsilon u_{x}^{2}\right) d x \leq C(\Omega) K_{2}^{2}(\Omega, \varepsilon) \quad \text { valid for } 0 \leq t \leq T \\
\sup _{0 \leq x \leq l-\delta}|u(x, t ; \varepsilon)| \leq C(\Omega) \varepsilon^{-\frac{1}{4}} K_{2}(\Omega, \varepsilon) \\
0 \leq t \leq T
\end{array}\right.
$$

with

$$
\begin{aligned}
\mathrm{K}_{2}(\Omega, \varepsilon)= & \|f\|_{\Omega}+\|\mathrm{g}\|_{[0, \ell]}+\left\|\mathrm{k}_{1}\right\|_{[0, \mathrm{~T}]}+\left\|\mathrm{k}_{2}\right\|_{[0, \mathrm{~T}]} \\
& +\varepsilon^{\frac{1}{2}}\left\|_{\mathrm{X}}\right\|_{[0, \ell]}+\varepsilon^{\frac{1}{2}}\left\|_{h}\right\|_{[0, \ell]}+\varepsilon^{\frac{1}{2}}\left\|_{\mathrm{k}_{\mathrm{t}}}\right\|_{[0, \mathrm{~T}]}+\varepsilon\left\|_{\mathrm{k}_{2}}\right\|_{[0, \mathrm{~T}]}
\end{aligned}
$$

and $\delta$ an arbitrarily small positive constant independent of $\varepsilon$ and satisfying $0<\delta<\ell$.

Similar refinements are possible if

$$
b(l, t ; \varepsilon) \leq-b_{0}<0 \quad \text { for } 0 \leq t \leq T \text { and } 0<\varepsilon \leq \varepsilon_{0} \text {. }
$$

\section{Approximations}

As an application of the a priori estimates that we derived in the preceding section we shall now construct an approximation for the solution $u(x, t ; \varepsilon)$ of the initial-boundary value problem

$$
\begin{cases}\varepsilon L_{2}[u]+L_{1}[u]=f(x, t), & (x, t) \in D \\ u(x, 0 ; \varepsilon)=g(x), & 0 \leq x \leq l \\ u_{t}(x, 0 ; \varepsilon)=h(x), & 0 \leq x \leq l \\ u(0, t ; \varepsilon)=k_{1}(t), & 0 \leq t<\infty \\ u(l, t ; \varepsilon)=k_{2}(t), & 0 \leq t<\infty\end{cases}
$$

where $0<\varepsilon<1$ and 


$$
\left\{\begin{array}{l}
L_{2}[u]=\frac{\partial^{2} u}{\partial t^{2}}-c^{2}(x, t) \frac{\partial^{2} u}{\partial x^{2}} \\
L_{1}[u]=a(x, t) \frac{\partial u}{\partial t}+b(x, t) \frac{\partial u}{\partial x}+d(x, t) u
\end{array}\right.
$$

In order not to complicate the calculations it will be assumed that all data are $\mathrm{C}^{\infty}$-functions which in addition satisfy

(29a) $\quad\left\{\begin{array}{l}a(x, t)>0, \quad c(x, t)>0 \\ 0<b(x, t)<a(x, t) c(x, t) \\ (29 c)\end{array} \quad\right.$ in $\bar{D} \bar{D}$
$g(0)=k_{1}(0), g(l)=k_{2}(0)$.

REMARK. The assumption $b(x, t)>0$ has been made for reasons of simplicity only. We emphasize that the results to be obtained in this section remain valid if less stringent conditions are imposed on the coefficient $b(x, t)$. These conditions will be specified at the end of this section. For the moment we confine ourselves to the remark that any problem with

$$
-a(x, t) c(x, t)<b(x, t)<0 \text { in } \bar{D}
$$

instead of (29b) can be reduced to a problem of the above mentioned type by means of a transformation of coordinates, namely $x^{\prime}=l-x, t^{\prime}=t$.

As a first approximation for the solution $u$ of (28) we could take the solution $w$ of the reduced problem

$$
\begin{cases}a(x, t) \frac{\partial w}{\partial t}+b(x, t) \frac{\partial w}{\partial x}+d(x, t) w=f(x, t), & (x, t) \in D \\ w(x, 0)=g(x), & 0 \leq x \leq l \\ w(0, t)=k_{1}(t), & 0 \leq t<\infty .\end{cases}
$$

However, the solution of this problem is generally not twice differentiable in $D$, so the quantity $L_{2}[w]$ cannot be evaluated. It will be clear that this difficulty has to be overcome before we can start with the construction of a formal approximation. 


\subsection{A lemma}

It is known that the solution of a problem of the type (30) belongs to the class $C^{n}(\bar{D})$ if the data satisfy certain compatibility conditions (see for instance DZAVADOV [8, p.1402]). In this subsection we state a condition which is necessary and sufficient for the solution w of problem (30) to be continuously differentiable in $\bar{D}$. A consequence is that under this condition the second derivatives of $\mathrm{w}$ (and hence also the expression $\mathrm{L}_{2}[\mathrm{w}]$ ) remain bounded in compact subsets of $\bar{D}$. In that case $w$ can be considered as a first approximation for the solution $u$ of $(28)$, since the quantity $\varepsilon L_{2}[w]$ is of order $\varepsilon$, uniformly in compact subsets of $\bar{D}$. The necessary and sufficient condition to be stated is closely related to the compatibility conditions mentioned before, but by the form in which it is written it has the advantage of being easier applicable. Since a proof of the correctness of compatibility conditions is not to be found in most text-books we shall for reasons of completeness state the results in the form of a lemma and give a detailed proof.

LEMMA 1. Let $\mathrm{u}(\mathrm{x}, \mathrm{y})$ be the solution of the boundary value problem

$$
\begin{cases}a(x, y) \frac{\partial u}{\partial x}+b(x, y) \frac{\partial u}{\partial y}+c(x, y) u=f(x, y), & (x, y) \in D \\ u(x, 0)=g(x), & 0 \leq x<\infty \\ u(0, y)=h(y), & 0 \leq y<\infty\end{cases}
$$

where the region $\mathrm{D}$ is given by

$$
D=\{(x, y) \mid 0<x, y<\infty\}
$$

and all data are supposed to be $\mathrm{C}^{\infty}$-functions which in addition satisfy the conditions

$$
\left\{\begin{array}{l}
a(x, y)>0, \quad b(x, y)>0 \quad \text { in } \bar{D} \\
g(0)=h(0) .
\end{array}\right.
$$

Then the function $\mathrm{u}(\mathrm{x}, \mathrm{y})$ belongs to $\mathrm{C}^{1}(\overline{\mathrm{D}})$ iff 


$$
a(0,0) g^{\prime}(0)+b(0,0) h^{\prime}(0)+c(0,0) g(0)=f(0,0) \text {. }
$$

In that case the second derivatives of $\mathrm{u}(\mathrm{x}, \mathrm{y})$ exist in $\overline{\mathrm{D}}$ and are continuous, with possible exception of the characteristic through the origin where the second derivative in the direction perpendicular to this characteristic may exhibit a finite jump.

PROOF. From the theory of first order partial differential equations it is known that the solution $u$ of (31) under the above mentioned conditions is infinitely differentiable in $\overline{\mathrm{D}}$, with the exception of the characteristic through the origin. Therefore we only have to investigate the behaviour of $\mathrm{u}$ in a neighborhood of this characteristic.

The necessity of the condition (32) follows immediately if we let the point $(x, y)$ tend to $(0,0)$ in the differential equation (31). In order to prove the sufficiency we shall first assume that $c(x, y) \equiv 0$ in $\bar{D}$. In that case problem (31) reduces to

$$
\begin{cases}a(x, y) \frac{\partial u}{\partial x}+b(x, y) \frac{\partial u}{\partial y}=f(x, y) & \text { in } D \\ u(x, 0)=g(x), & 0 \leq x<\infty \\ u(0, y)=h(y), & 0 \leq y<\infty .\end{cases}
$$

Let $\tilde{a}(x, y)$ and $\tilde{b}(x, y)$ be positive functions defined in

$$
\overline{\mathrm{D}} \cup\{(\mathrm{x}, \mathrm{y}) \mid-\infty<\mathrm{x}<0,0 \leq \mathrm{y} \leq 2\}
$$

which satisfy

$$
\tilde{a}(x, y) \equiv a(x, y), \quad \tilde{b}(x, y) \equiv b(x, y) \quad \text { in } \bar{D} \text {. }
$$

It is possible to choose these functions such that they are $\mathrm{n}$ times continuously differentiable ( $\mathrm{n}$ an arbitrarily large positive integer). A possible choice for $\tilde{a}(x, y)$ is for instance

$$
\tilde{a}(x, y)= \begin{cases}\Psi(-3 \delta-x)+\psi(x) \sum_{k=0}^{n} \frac{x^{k}}{k !} \frac{\partial^{k} a}{\partial x^{k}}(0, y) & \text { for }-\infty<x \leq 0,0 \leq y \leq 2 \\ a(x, y) & \text { for } 0 \leq x, y<\infty\end{cases}
$$


where $\delta$ is a sufficiently small positive constant and $\Psi(x)$ is some $C^{\infty}$-function defined in $\mathbb{R}$ which satisfies

$$
\begin{cases}\Psi(x)= \begin{cases}1 & \text { for } x \geq-\delta \\ 0 & \text { for } x \leq-2 \delta\end{cases} \\ \Psi(x) \text { monotonically increasing for }-2 \delta \leq x \leq-\delta .\end{cases}
$$

A similar choice is possible for $\tilde{b}(x, y)$.

Let $x=\Phi_{1}(y), y \geq 0$ denote the monotonically increasing solution of

$$
\frac{d x}{d y}=\frac{\tilde{a}(x, y)}{\tilde{b}(x, y)}, \quad x(1)=0 .
$$

The graph of this solution intersects the $x$-axis at $x=-x_{0}$ (see figure 3 ). Let $\mathrm{x}=\Phi_{2}(\mathrm{y}), \mathrm{y} \geq 0$ denote the monotonically increasing solution of

$$
\frac{d x}{d y}=\frac{a(x, y)}{b(x, y)}, \quad x(0)=x_{0}
$$

Finally, let $x=\Phi(y), y \geq 0$ denote the solution of

$$
\frac{d x}{d y}=\frac{a(x, y)}{b(x, y)}, \quad x(0)=0
$$

This function is likewise monotonically increasing for $y \geq 0$ (see figure 3 ). We are going to apply a coordinate transformation to the region $\Omega$ defined by

$$
\left\{\begin{array}{l}
\Omega_{-}=\Omega_{-} \cup \Omega_{+} \\
\Omega_{-}=\left\{(\mathrm{x}, \mathrm{y}) \mid \max \left(0, \Phi_{1}(\mathrm{y})\right)<\mathrm{x} \leq \Phi(\mathrm{y}), \mathrm{y}>0\right\} \\
\Omega_{+}=\left\{(\mathrm{x}, \mathrm{y}) \mid \Phi(\mathrm{y}) \leq \mathrm{x}<\Phi_{2}(\mathrm{y}), \mathrm{y}>0\right\}
\end{array}\right.
$$

(see figure 3). To this end we introduce functions $\phi$ and $\psi$ which are the solutions of the boundary value problems 
(34)

$$
\left\{\begin{array}{l}
\tilde{a}(x, y) \frac{\partial \phi}{\partial x}+\tilde{b}(x, y) \frac{\partial \phi}{\partial y}=0, \quad \Phi_{1}(y)<x<\Phi_{2}(y), y>0 \\
\phi(x, 0)=x, \quad-x_{0} \leq x \leq x_{0}
\end{array}\right.
$$

and

$$
\left\{\begin{array}{l}
b(x, y) \frac{\partial \psi}{\partial x}-a(x, y) \frac{\partial \psi}{\partial y}=0 \quad \text { in } \Omega \\
\psi(\Phi(y), y)=y, \quad y \geq 0 .
\end{array}\right.
$$

Note that the functions $\Phi, \Phi_{2}$ and $\psi$ are $C^{\infty}$-functions. The functions $\Phi_{1}$ and $\phi$ can be made arbitrarily smooth by choosing the coefficients $\tilde{a}$ and $\tilde{b}$ sufficiently smooth.

We define new coordinates $\xi$ and $n$ by

$$
\begin{cases}\xi=\phi(x, y) & \text { for }(x, y) \in \bar{\Omega} \\ \eta=\psi(x, y) & \text { for }(x, y) \in \bar{\Omega}\end{cases}
$$

This transformation transforms the characteristics of the operator

$$
a(x, y) \frac{\partial}{\partial x}+b(x, y) \frac{\partial}{\partial y}
$$

and their orthogonal trajectories into lines $\xi=$ constant and $\eta=$ constant. The characteristic through the origin is transformed into the line $\xi=0$ (see figure 3 ).

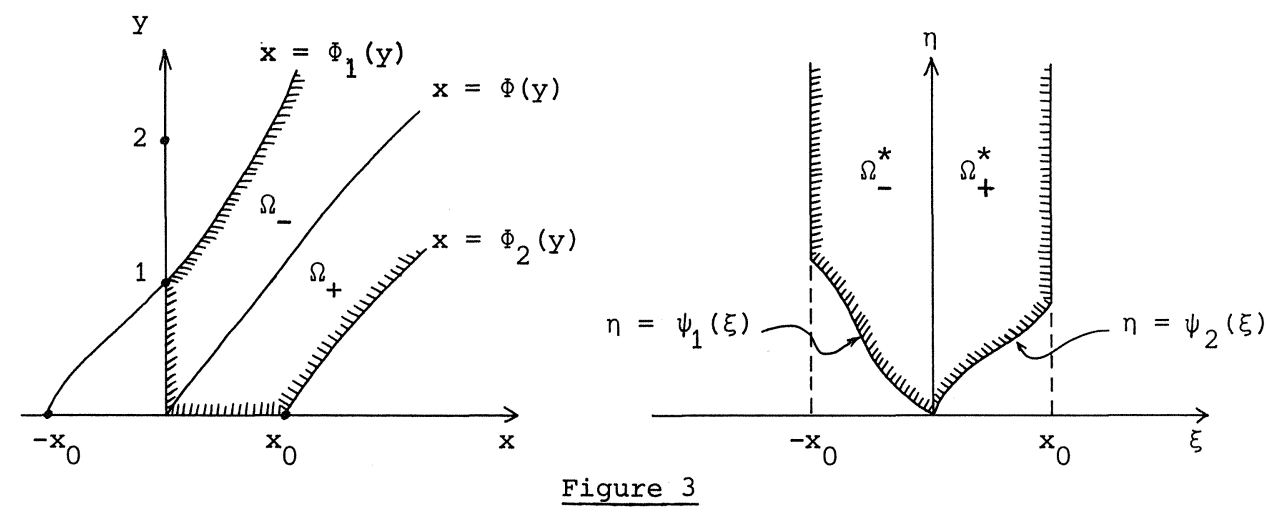

\section{Figure 3}


Problem (33) (restricted to the region $\bar{\Omega}$ ) becomes after the above mentioned transformation

(36)

$$
\begin{cases}\left(a \psi_{x}+b \psi_{y}\right) \frac{\partial u}{\partial \eta}=f & \text { for }(\xi, \eta) \in \Omega^{*} \\ u\left(\xi, \psi_{1}(\xi)\right)=h\left(\phi_{1}^{-1}(\xi)\right), & -x_{0} \leq \xi \leq 0 \\ u\left(\xi, \psi_{2}(\xi)\right)=g\left(\phi_{2}^{-1}(\xi)\right), & 0 \leq \xi \leq x_{0}\end{cases}
$$

where the region $\Omega^{*}$ is given by

$$
\left\{\begin{array}{l}
\Omega^{*}=\Omega_{-}^{*} \cup \Omega_{+}^{*} \\
\Omega_{-}^{*}=\left\{(\xi, n) \mid-x_{0}<\xi \leq 0, n>\psi_{1}(\xi)\right\} \\
\Omega_{+}^{*}=\left\{(\xi, \eta) \mid 0 \leq \xi<x_{0}, \eta>\psi_{2}(\xi)\right\} .
\end{array}\right.
$$

The function $\phi_{1}^{-1}$ is the inverse of the monotonically decreasing function $\phi_{1}(\mathrm{y})=\phi(0, \mathrm{y}), 0 \leq \mathrm{y} \leq 1$; the function $\phi_{2}^{-1}$ is the inverse of the monotonically increasing function $\phi_{2}(x)=\phi(x, 0), 0 \leq x \leq x_{0}$. The functions $\psi_{1}$ and $\psi_{2}$ (monotonically decreasing for $-x_{0} \leq \xi \leq 0$ resp. monotonically increasing for $0 \leq \xi \leq x_{0}$ ) are defined by

$$
\begin{cases}\dot{\psi}_{1}(\xi)=\psi\left(0, \phi_{1}^{-1}(\xi)\right), & -x_{0} \leq \xi \leq 0 \\ \psi_{2}(\xi)=\psi\left(\phi_{2}^{-1}(\xi), 0\right), & 0 \leq \xi \leq x_{0} .\end{cases}
$$

Since $a \psi_{x}+b \psi_{y} \neq 0$ in $\bar{D}$ we obtain from (36) the solution

$$
u(\xi, n)= \begin{cases}\int_{\psi_{1}(\xi)}^{\eta}\left(\frac{f}{a \psi_{x}+b \psi_{y}}\right)(\xi, \tau) d \tau+h\left(\phi_{1}^{-1}(\xi)\right) & \text { in } \overline{\Omega_{-}^{*}} \\ \int_{\psi_{2}(\xi)}^{\eta}\left(\frac{f}{a \psi_{x}+b \psi_{y}}\right)(\xi, \tau) d \tau+g\left(\phi_{2}^{-1}(\xi)\right) & \text { in } \overline{\Omega_{+}^{*}} .\end{cases}
$$

From this formula it follows immediately that $u$ is continuous in $\overline{\Omega^{*}}$ and infinitely differentiable with respect to $n$. It also follows that $u$ is $n$ times differentiable with respect to $\xi$, provided that $\xi \neq 0$ (n an arbitrarily 
large positive integer). We obtain in $\overline{\Omega_{-}^{*}}$

$$
\frac{\partial u}{\partial \xi}=\int_{\psi_{1}(\xi)}^{\eta} \frac{\partial}{\partial \xi}\left(\frac{f}{a \psi_{x}+b \psi_{y}}\right)(\xi, \tau) d \tau+\frac{h^{\prime}\left(\phi_{1}^{-1}(\xi)\right)}{\phi_{y}\left(0, \phi_{1}^{-1}(\xi)\right)}
$$

(38)

$$
-\frac{\psi_{y}\left(0, \phi_{1}^{-1}(\xi)\right)}{\phi_{y}\left(0, \phi_{1}^{-1}(\xi)\right)}\left(\frac{f}{a \psi_{x}+b \psi_{y}}\right)\left(\xi, \psi_{1}(\xi)\right)
$$

and in $\overline{\Omega_{+}^{*}}$

$$
\frac{\partial u}{\partial \xi}=\int_{\psi_{2}}^{\eta} \frac{\partial}{\partial \xi}\left(\frac{f}{a \psi_{x}+b \psi_{y}}\right)(\xi, \tau) d \tau+\frac{g^{\prime}\left(\phi_{2}^{-1}(\xi)\right)}{\phi_{x}\left(\phi_{2}^{-1}(\xi), 0\right)}
$$

(39)

$$
-\frac{\psi_{x}\left(\phi_{2}^{-1}(\xi), 0\right)}{\phi_{x}\left(\phi_{2}^{-1}(\xi), 0\right)}\left(\frac{f}{a \psi_{x}+b \psi_{y}}\right)\left(\xi, \psi_{2}(\xi)\right) .
$$

Using the smoothness of the solutions $\phi$ and $\psi$ we obtain from (34) and (35) after a straightforward calculation

$$
\left\{\begin{array}{l}
\phi_{X}(0,0)=1 \\
\phi_{Y}(0,0)=-\frac{a(0,0)}{b(0,0)} \\
\psi_{X}(0,0)=\frac{a(0,0) b(0,0)}{a^{2}(0,0)+b^{2}(0,0)} \\
\psi_{Y}(0,0)=\frac{b^{2}(0,0)}{a^{2}(0,0)+b^{2}(0,0)}
\end{array}\right.
$$

Hence it follows from (38) and (39) that

$$
\begin{aligned}
\frac{\partial u}{\partial \xi}(+0, n)-\frac{\partial u}{\partial \xi}(-0, n)= & {\left[\frac{\psi_{y}(0,0)}{\phi_{y}(0,0)}-\frac{\psi_{x}(0,0)}{\phi_{x}(0,0)}\right]\left(\frac{f}{a \psi_{x}+b \psi_{y}}\right)(0,0) } \\
& +\frac{g^{\prime}(0)}{\phi_{x}(0,0)}-\frac{h^{\prime}(0)}{\phi_{y}(0,0)} \\
= & \frac{1}{a(0,0)}\left\{a(0,0) g^{\prime}(0)+b(0,0) h^{\prime}(0)-f(0,0)\right\} \\
= & 0
\end{aligned}
$$


because of $(32)$ (with $c(0,0)=0$ ); so $\frac{\partial u}{\partial \xi}$ is continuous in $\overline{\Omega^{*}}$.

It has now been shown that $u_{\xi}$ and $u_{\eta}$ are continuous in $\bar{\Omega}$, whence it follows that $u_{x}$ and $u_{y}$ are continuous in $\bar{\Omega}$. Thus we have established the sufficiency of (32) in case $c(x, y) \equiv 0$ in $\bar{D}$. The other statements of the lemma follow directly from (38) and (39) by differentiation; in particular, it follows that the second derivative $u_{\xi \xi}$ in the direction perpendicular to the characteristic through the origin may exhibit a finite jump across this characteristic. The second derivatives $u_{\xi \eta}$ and $u_{\eta \eta}$ are continuous in $\overline{\Omega^{*}}$.

If $c(x, y) \not \equiv 0$ we put

$$
u=w e^{v}
$$

where $\mathrm{w}$ and $\mathrm{v}$ are the solutions of

$$
\left\{\begin{array}{l}
a(x, y) \frac{\partial v}{\partial x}+b(x, y) \frac{\partial v}{\partial y}=-c(x, y), \quad(x, y) \in D \\
v(x, 0)=-\frac{c(0,0)}{a(0,0)} x, \quad 0 \leq x<\infty \\
v(0, y)=0, \quad 0 \leq y<\infty
\end{array}\right.
$$

and

$$
\begin{cases}a(x, y) \frac{\partial w}{\partial x}+b(x, y) \frac{\partial w}{\partial y}=f(x, y) \exp [-v(x, y)], & (x, y) \in D \\ w(x, 0)=g(x) \exp \left[\frac{c(0,0)}{a(0,0)} x\right], \quad 0 \leq x<\infty \\ w(0, y)=h(y), \quad 0 \leq y<\infty .\end{cases}
$$

These boundary value problems are both of the type (33). It follows from the preceding analysis that $v(x, y) \in C^{1}(\bar{D})$ and also, because of the condition (32), that $w(x, y) \in C^{1}(\bar{D})$ (note that the function $f(x, y) \exp [-v(x, y)]$ in $(40)$ does not belong to $C^{\infty}(\bar{D})$ but has second derivatives which may exhibit a finite jump across the characteristic through the origin; one easily verifies that this does not affect the proof that we gave for the case $c(x, y) \equiv 0)$. Hence we obtain $u(x, y) \in C^{1}(\bar{D})$ which completes the proof of the lemma. 
REMARK. The condition of positiveness of the coefficients $a(x, y)$ and $b(x, y)$ can be disposed of. It can be shown in a similar way as before that the statements of the lemma remain valid if instead of this condition the following requirements are fulfilled:

(i) $\quad a^{2}(x, y)+b^{2}(x, y) \neq 0$ in $\bar{D}$

(ii) the characteristics are nowhere tangent to the boundary, so $a(0, y) \neq 0$ for $y \geq 0$ and $b(x, 0) \neq 0$ for $x \geq 0$

(iii) the characteristic through the origin has a positive slope in $(0,0)$, so $a(0,0) / b(0,0)>0$

(iv) through each point in $\bar{D}$ there passes exactly one characteristic and this characteristic has exactly one point in common with the boundary of $\mathrm{D}$.

Also the condition of infinite smoothness of the data can be weakened.

Now that we have established a condition which is necessary and sufficient in order that the solution w of the reduced problem (30) has bounded second derivatives we can start with the construction of a formal approximation. We might of course impose a compatibility condition of the type (32) on the singular perturbation problem (28) and then take the solution $w$ of (30) as the first term of the formal approximation. However, it is also possible to construct an approximation for the solution $u$ of (28) without imposing such a condition. This construction is carried out in the next subsection.

\subsection{Construction of an approximation}

We consider instead of (30) the so-called modified reduced problem

$$
\left\{\begin{array}{lc}
a(x, t) \frac{\partial \tilde{w}}{\partial t}+b(x, t) \frac{\partial \tilde{w}}{\partial x}+d(x, t) \tilde{w}=f(x, t), & (x, t) \in D \\
\tilde{w}(x, 0 ; \varepsilon)=g(x), & 0 \leq x \leq \ell \\
\tilde{w}(0, t ; \varepsilon)=K(t ; \varepsilon), & 0 \leq t<\infty
\end{array}\right.
$$

where the function $K(t ; \varepsilon)$ is defined by

$$
K(t ; \varepsilon)=k_{1}(t)-C t \exp \left(-\frac{t}{\varepsilon^{p}}\right), \quad 0 \leq t<\infty,
$$


the constant $\mathrm{C}$ is given by

$$
C=\frac{a(0,0) k_{1}^{\prime}(0)+b(0,0) g^{\prime}(0)+d(0,0) g(0)-f(0,0)}{a(0,0)}
$$

and $\mathrm{p}$ is some positive constant to be determined later on.

Note that the expression $L_{2}[\tilde{w}]$ is defined because it follows from lemma 1 that the solution $\tilde{w}(x, t ; \varepsilon)$ of $(41)$ is twice differentiable. Moreover, (41) differs only slightly from (30) because of

$$
\sup _{0 \leq t<\infty} t \exp \left(-\frac{t}{\varepsilon^{p}}\right)=0\left(\varepsilon^{p}\right), \quad \varepsilon+0
$$

so we may expect the difference between $\tilde{w}$ and $w$ to be small, too. Therefore it is reasonable to take the solution $\tilde{w}$ of the modified reduced problem (41) as a first approximation for the solution $u$ of (28).

In order to cope with the remaining boundary conditions in (28) we shall look for an approximation $\tilde{u}(x, t ; \varepsilon)$ of $u(x, t ; \varepsilon)$ in the form

$$
\tilde{u}(x, t ; \varepsilon)=\tilde{w}(x, t ; \varepsilon)+v_{1}(\xi, t ; \varepsilon)+\varepsilon\left\{v_{2}(\xi, t ; \varepsilon)+v_{3}(x, \tau ; \varepsilon)\right\}
$$

where

$$
\xi=\frac{l-x}{\varepsilon}, \tau=\frac{t}{\varepsilon}
$$

Expanding the operator $L^{(\varepsilon)}=\varepsilon L_{2}+L_{1}$ into powers of $\varepsilon$ we obtain

$$
\begin{aligned}
L^{(\varepsilon)}[\tilde{u}]= & f(x, t)-\varepsilon^{-1}\left\{c^{2}(l, t) \frac{\partial^{2} v_{1}}{\partial \xi^{2}}+b(l, t) \frac{\partial v_{1}}{\partial \xi}\right\} \\
& -\left\{c^{2}(l, t) \frac{\partial^{2} v_{2}}{\partial \xi^{2}}+b(l, t) \frac{\partial v_{2}}{\partial \xi}-\phi(\xi, t ; \varepsilon)\right\} \\
& +\left\{\frac{\partial^{2} v_{3}}{\partial \tau^{2}}+a(x, 0) \frac{\partial v_{3}}{\partial \tau}\right\}+\varepsilon \psi(x, t ; \varepsilon) \\
& +\varepsilon^{2}\left\{\frac{\partial^{2} v_{2}}{\partial t^{2}}-c^{2}(x, t) \frac{\partial^{2} v_{3}}{\partial x^{2}}\right\}
\end{aligned}
$$

where 


$$
\phi(\xi, t ; \varepsilon)=2 \xi\left(c c_{x}\right)(l, t) \frac{\partial^{2} v_{1}}{\partial \xi^{2}}+a(l, t) \frac{\partial v_{1}}{\partial t}+\xi b_{x}(l, t) \frac{\partial v_{1}}{\partial \xi}+d(l, t) v_{1}
$$

and

$$
\begin{aligned}
\psi(x, t ; \varepsilon)= & \frac{\partial^{2} \tilde{w}}{\partial t^{2}}-c^{2}(x, t) \frac{\partial \tilde{w}}{\partial x^{2}}+\frac{\partial^{2} v_{1}}{\partial t^{2}}-\xi^{2}\left(c c_{x}\right)\left(l+\Theta_{1}(x-\ell), t\right) \frac{\partial^{2} v_{1}}{\partial \xi^{2}} \\
& -\xi a_{x}\left(l+\Theta_{2}(x-l), t\right) \frac{\partial v_{1}}{\partial t}-\frac{1}{2} \xi^{2} b_{x x}\left(l+\Theta_{3}(x-l), t\right) \frac{\partial v_{1}}{\partial \xi} \\
& -\xi a_{x}\left(l+\theta_{4}(x-\ell), t\right) v_{1}+2 \xi\left(c c_{x}\right)\left(l+\theta_{5}(x-l), t\right) \frac{\partial^{2} v_{2}}{\partial \xi^{2}} \\
& +a(x, t) \frac{\partial v_{2}}{\partial t}+\xi b_{x}\left(l+\Theta_{6}(x-\ell), t\right) \frac{\partial v_{2}}{\partial \xi}+d(x, t) v_{2} \\
& +\tau a_{t}\left(x, \theta_{7} t\right) \frac{\partial v_{3}}{\partial \tau}+b(x, t) \frac{\partial v_{3}}{\partial x}+d(x, t) v_{3}
\end{aligned}
$$

with $0<\theta_{i}<1, i=1,2, \ldots, 7$.

In addition we have

$$
(46) \begin{cases}\tilde{u}(x, 0 ; \varepsilon)=g(x)+v_{1}(\xi, 0 ; \varepsilon)+\varepsilon\left\{v_{2}(\xi, 0 ; \varepsilon)+v_{3}(x, 0 ; \varepsilon)\right\}, & 0 \leq x \leq l \\ \tilde{u}_{t}(x, 0 ; \varepsilon)=\tilde{w}_{t}(x, 0 ; \varepsilon)+\frac{\partial v_{1}}{\partial t}(\xi, 0 ; \varepsilon)+\varepsilon \frac{\partial}{\partial t}(\xi, 0 ; \varepsilon)+\frac{\partial v_{3}}{\partial \tau}(x, 0 ; \varepsilon), & 0 \leq x \leq l \\ \tilde{u}(0, t ; \varepsilon)=k(t ; \varepsilon)+v_{1}\left(\frac{l}{\varepsilon}, t ; \varepsilon\right)+\varepsilon\left\{v_{2}\left(\frac{l}{\varepsilon}, t ; \varepsilon\right)+v_{3}(0, \tau ; \varepsilon)\right\}, & 0 \leq t<\infty \\ \tilde{u}(l, t ; \varepsilon)=\tilde{w}(l, t ; \varepsilon)+v_{1}(0, t ; \varepsilon)+\varepsilon\left\{v_{2}(0, t ; \varepsilon)+v_{3}(l, \tau ; \varepsilon)\right\}, & 0 \leq t<\infty .\end{cases}
$$

We shall now specify the functions $v_{i}(i=1,2,3)$. For the function $v_{1}$ we take the solution of

$$
\left\{\begin{array}{l}
c^{2}(\ell, t) \frac{\partial^{2} v_{1}}{\partial \xi^{2}}+b(l, t) \frac{\partial v_{1}}{\partial \xi}=0, \quad 0<\xi<\infty, \quad 0 \leq t<\infty \\
v_{1}(0, t ; \varepsilon)=k_{2}(t)-\tilde{w}(l, t ; \varepsilon)+\theta \Phi\left(t-t_{0} ; \varepsilon\right), \quad 0 \leq t<\infty \\
\lim _{\xi \rightarrow \infty} v_{1}(\xi, t ; \varepsilon)=0, \quad 0 \leq t<\infty
\end{array}\right.
$$


where $t_{0}$ is the $t$-coordinate of the point of intersection between the line $x=l$ and the subcharacteristic through the origin (see figure 4 ), the $\varepsilon-$ dependent constant $\theta$ is given by

$$
\theta=\frac{1}{2}\left\{\frac{\partial^{2} \tilde{w}}{\partial t^{2}}\left(l, t_{0}+0 ; \varepsilon\right)-\frac{\partial^{2} \tilde{w}}{\partial t^{2}}\left(l, t_{0}-0 ; \varepsilon\right)\right\}
$$

and the function $\Phi(t ; \varepsilon)$ is defined by

$$
\Phi(t ; \varepsilon)= \begin{cases}\frac{1}{2} t^{2} \exp \left(-\frac{t}{\varepsilon}\right), & t \geq 0 \\ -\frac{1}{2} t^{2} \exp \left(\frac{t}{\varepsilon}\right), & t \leq 0\end{cases}
$$

with $\mathrm{q}$ some positive constant to be determined later on.

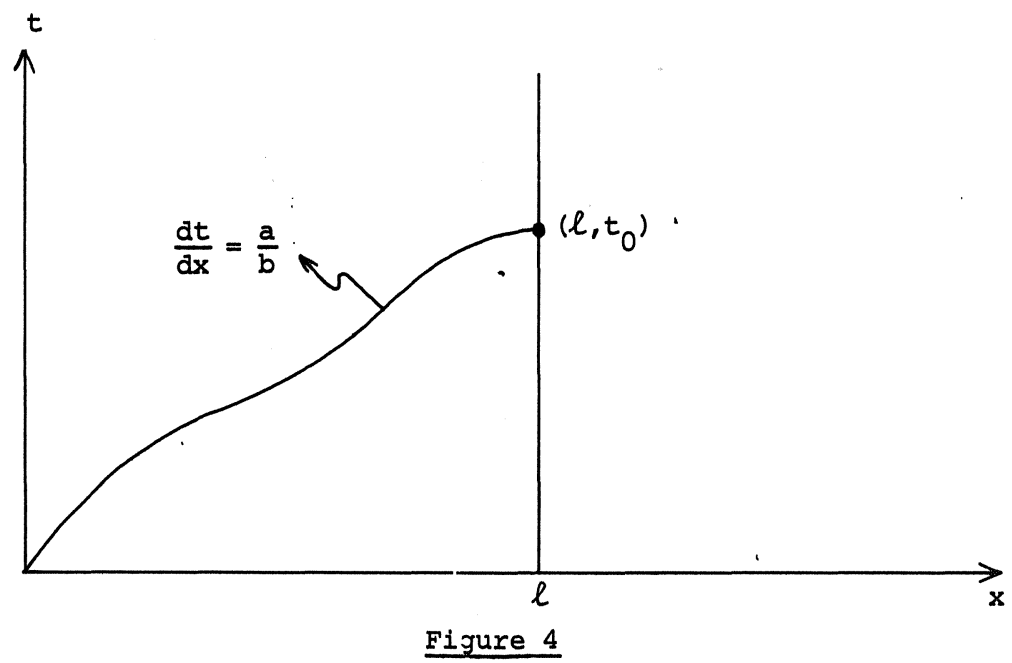

It will turn out in the course of the analysis that the prescribed boundary function $v_{1}(0, t ; \varepsilon)$ should be at least three times differentiable. Therefore the term $\Theta \Phi\left(t-t_{0} ; \varepsilon\right)$ in (47) cannot be omitted, since the remaining function $k_{2}(t)-\tilde{w}(l, t ; \varepsilon)$ is only twice differentiable. One easily verifies that the boundary function

$$
k_{2}(t)-\tilde{w}(l, t ; \varepsilon)+\theta \Phi\left(t-t_{0} ; \varepsilon\right)
$$

is indeed three times differentiable. 
It follows from (47) that

$$
v_{1}(\xi, t ; \varepsilon)=\left\{k_{2}(t)-\tilde{w}(l, t ; \varepsilon)+\theta \Phi\left(t-t_{0} ; \varepsilon\right)\right\} \exp \left[-\frac{b(l, t)}{c^{2}(l, t)} \xi\right]
$$

valid for $0 \leq \xi, t<\infty$.

REMARK. If the subcharacteristic through the origin does not intersect the line $\mathrm{x}=l$ the analysis can be simplified. We leave the details to the interested reader.

For the function $v_{2}$ we take the solution of

$$
\left\{\begin{array}{l}
c^{2}(\ell, t) \frac{\partial^{2} v_{2}}{\partial \xi^{2}}+b(\ell, t) \frac{\partial v_{2}}{\partial \xi}=\phi(\xi, t ; \varepsilon), \quad 0<\xi<\infty, 0 \leq t<\infty \\
v_{2}(0, t ; \varepsilon)=\lim _{\xi \rightarrow \infty} v_{2}(\xi, t ; \varepsilon)=0, \quad 0 \leq t<\infty .
\end{array}\right.
$$

Using (44) and (50) we can write this problem as

$$
\begin{cases}\frac{\partial^{2} v_{2}}{\partial \xi^{2}}+\frac{b(l, t)}{c^{2}(l, t)} \frac{\partial v_{2}}{\partial \xi}=\left\{\frac{\alpha(t ; \varepsilon)}{c^{2}(l, t)} \xi+\frac{\beta(t ; \varepsilon)}{c^{2}(l, t)}\right\} \exp \left[-\frac{b(l, t)}{c^{2}(l, t)} \xi\right], & \begin{array}{l}
0<\xi<\infty, \\
0 \leq t<\infty
\end{array} \\
v_{2}(0, t ; \varepsilon)=\lim _{\xi \rightarrow \infty} v_{2}(\xi, t ; \varepsilon)=0, & 0 \leq t<\infty\end{cases}
$$

with

$$
\left\{\begin{aligned}
\alpha(t ; \varepsilon)= & \left\{\tilde{w}(l, t ; \varepsilon)-\theta \Phi\left(t-t_{0} ; \varepsilon\right)-k_{2}(t)\right\} \times \\
& \times\left\{a(l, t) \frac{d}{d t} \frac{b(l, t)}{c^{2}(l, t)}+\frac{b(l, t) b_{x}(l, t)}{c^{2}(l, t)}-\frac{2\left(c c_{x}\right)(l, t) b^{2}(l, t)}{c^{4}(l, t)}\right\} \\
\beta(t ; \varepsilon)= & d(l, t)\left\{k_{2}(t)-\tilde{w}(l, t ; \varepsilon)+\theta \Phi\left(t-t_{0} ; \varepsilon\right)\right\} \\
& +a(l, t)\left\{k_{2}^{\prime}(t)-\tilde{w}_{t}(l, t ; \varepsilon)+\theta \Phi^{\prime}\left(t-t_{0} ; \varepsilon\right)\right\} .
\end{aligned}\right.
$$

Hence we obtain 


$$
v_{2}(\xi, t ; \varepsilon)=\left\{-\frac{\alpha(t ; \varepsilon)}{2 b(l, t)} \xi^{2}-\frac{\alpha(t ; \varepsilon) c^{2}(l, t)+\beta(t ; \varepsilon) b(l, t)}{b^{2}(l, t)} \xi\right\} \times
$$

$$
\times \exp \left[-\frac{b(\ell, t)}{c^{2}(\ell, t)} \xi\right]
$$

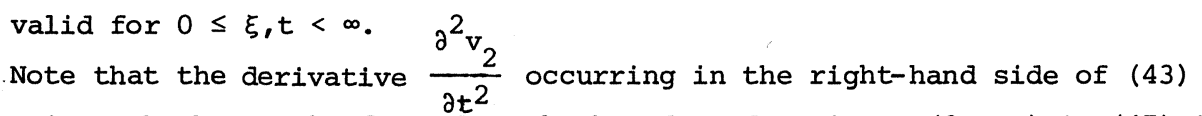
exists thanks to the fact that the boundary function $v_{1}(0, t ; \varepsilon)$ in $(47)$ is three times differentiable..

Finally, we take for the function $v_{3}$ the solution of

$$
\left\{\begin{array}{l}
\frac{\partial^{2} v_{3}}{\partial \tau^{2}}+a(x, 0) \frac{\partial v_{3}}{\partial \tau}=0, \quad 0 \leq x \leq l, 0<\tau<\infty \\
\frac{\partial v_{3}}{\partial \tau}(x, 0 ; \varepsilon)=h(x)-\tilde{w}_{t}(x, 0 ; \varepsilon), \quad 0 \leq x \leq l \\
\lim _{\tau \rightarrow \infty} v_{3}(x, \tau ; \varepsilon)=0, \quad 0 \leq x \leq l
\end{array}\right.
$$

which is given by

$$
v_{3}(x, \tau ; \varepsilon)=\frac{\tilde{w}_{t}(x, 0 ; \varepsilon)-h(x)}{a(x, 0)} \exp [-a(x, 0) \tau], \quad 0 \leq x \leq \ell, 0 \leq \tau<\infty .
$$

Note that $v_{1}, v_{2}$ and $v_{3}$ are boundary layer functions.

It follows from (43), (46), (47), (50), (51) and (53) that

(55)

$$
\left\{\begin{array}{l}
\mathrm{L}^{(\varepsilon)}[\tilde{u}]=f(x, t)+\varepsilon \psi(x, t ; \varepsilon)+\varepsilon^{2}\left\{\frac{\partial^{2} v_{2}}{\partial t^{2}}-c^{2}(x, t) \frac{\partial^{2} v_{3}}{\partial x^{2}}\right\}, \quad(x, t) \in D \\
\tilde{u}(x, 0 ; \varepsilon)=g(x)+\varepsilon\left\{v_{2}(\xi, 0 ; \varepsilon)+v_{3}(x, 0 ; \varepsilon)\right\} \\
\quad+\theta \Phi\left(-t_{0} ; \varepsilon\right) \exp \left[-\frac{b(l, 0)}{c^{2}(l, 0)} \xi\right], \quad 0 \leq x \leq l \\
\tilde{u}_{t}(x, 0 ; \varepsilon)=h(x)+\frac{\partial v_{1}}{\partial t}(\xi, 0 ; \varepsilon)+\varepsilon \frac{\partial v_{2}}{\partial t}(\xi, 0 ; \varepsilon), \quad 0 \leq x \leq l \\
\tilde{u}(0, t ; \varepsilon)=k(t ; \varepsilon)+v_{1}\left(\frac{l}{\varepsilon}, t ; \varepsilon\right)+\varepsilon\left\{v_{2}\left(\frac{l}{\varepsilon}, t ; \varepsilon\right)+v_{3}(0, \tau ; \varepsilon)\right\}, 0 \leq t<\infty \\
\tilde{u}(l, t ; \varepsilon)=k_{2}(t)+\varepsilon v_{3}(l, \tau ; \varepsilon)+\theta \Phi\left(t-t_{0} ; \varepsilon\right), \quad 0 \leq t<\infty .
\end{array}\right.
$$


Defining the remainder term $z(x, t ; \varepsilon)$ by

$$
z(x, t ; \varepsilon)=u(x, t ; \varepsilon)-\tilde{u}(x, t ; \varepsilon)
$$

we obtain from (28) and (55)

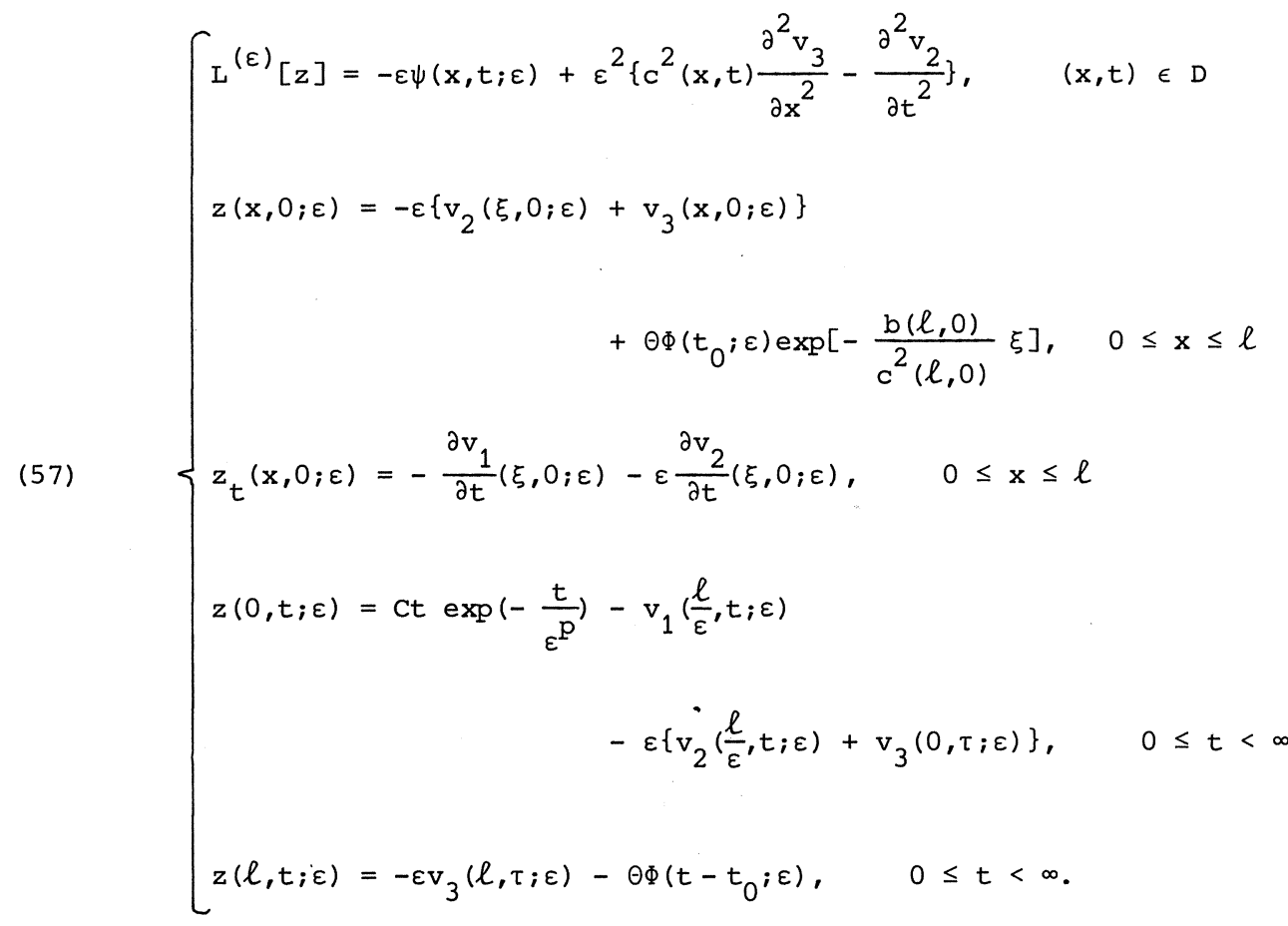

By writing the solution $\tilde{w}$ of (41) in explicit form (af. the proof of lemma 1) it is easily established that the $\varepsilon$-dependence of $\tilde{w}$ and its derivatives is completely determined by the $\varepsilon$-dependence of the boundary function $\mathrm{K}(t ; \varepsilon)$ and $i$ ts derivatives (cf. the formulae (37), (38) and (39)). Bearing this in mind we obtain after tedious calculations

$$
\begin{cases}\left\|\frac{\partial^{2 \tilde{w}}}{\partial t^{2}}\right\|_{\Omega}=O\left(\varepsilon^{-\frac{1}{2} p}\right), & \varepsilon \downarrow 0 \\ \left\|\frac{\partial^{2 \tilde{w}}}{\partial x^{2}}\right\|_{\Omega}=O\left(\varepsilon^{-\frac{1}{2} p}\right), & \varepsilon \downarrow 0 \\ \sup _{\Omega}\left|\frac{\partial^{2} \tilde{w}}{\partial t^{2}}\right|=0\left(\varepsilon^{-p}\right), & \varepsilon \downarrow 0\end{cases}
$$


valid for any compact subset $\Omega \subset \overline{\mathrm{D}}$.

Similarly we obtain from (50) and (52) the results

$$
\begin{cases}\left\|\frac{\partial^{2} v_{1}}{\partial t^{2}}\right\|_{\Omega}=O\left(\varepsilon^{\lambda_{1}}\right), & \varepsilon \downarrow 0 \\ \left\|\frac{\partial^{2} v_{2}}{\partial t^{2}}\right\|_{\Omega}=O\left(\varepsilon^{\lambda_{2}}\right), & \varepsilon \downarrow 0\end{cases}
$$

valid for any compact subset $\Omega \subset \bar{D}$, where

$$
\left\{\begin{array}{l}
\lambda_{1}=\min \left(-\frac{1}{2} p,-p+\frac{1}{2} q\right) \\
\lambda_{2}=\min \left(-\frac{3}{2} p,-p-\frac{1}{2} q\right) .
\end{array}\right.
$$

Hence we obtain from (45)

$$
\|\psi\|_{\Omega}=O\left(\varepsilon^{\lambda}\right), \quad \varepsilon+0
$$

valid for any compact subset $\Omega \subset \bar{D}$.

Applying theorem 1 (and the second remark following that theorem) to (57) it is possible to obtain estimates for the function $z$ and its derivatives. Pointwise estimates for $z$ follow from the formulae (24a), (25) and (26b), (27). Using the formulae (58), (59) and (60) it can be shown that the constants $K(\Omega, \varepsilon)$ and $K_{2}(\Omega, \varepsilon)$ defined by (25) and (27) satisfy

$$
\begin{cases}\mathrm{K}(\Omega, \varepsilon)=0\left(\varepsilon^{\mu}\right), & \varepsilon \downarrow 0 \\ \mathrm{~K}_{2}(\Omega, \varepsilon)=0\left(\varepsilon^{\mu}\right), & \varepsilon \downarrow 0\end{cases}
$$

with

$$
\mu=\min \left(1-\frac{1}{2} p, 1-p+\frac{1}{2} q, 2-\frac{3}{2} p, 2-p-\frac{1}{2} q, \frac{3}{2} p,-p+\frac{5}{2} q\right) .
$$

It is not difficult to show that the value of $\mu$ becomes optimal (that is, maximal) if we choose $\mathrm{p}=\mathrm{q}=\frac{1}{2}$. In that case we obtain $\mu=\frac{3}{4}$, so that according to the formulae $(24 a)$ and $(26 b)$ 
(61)

$$
\left\{\begin{array}{l}
\sup _{0 \leq x \leq \ell}|z(x, t ; \varepsilon)|=0\left(\varepsilon^{\frac{1}{4}}\right), \quad \varepsilon+0 \\
0 \leq t \leq T \\
\sup _{\substack{0 \leq x \leq \ell-\delta \\
0 \leq t \leq T}}|z(x, t ; \varepsilon)|=0\left(\varepsilon^{\frac{1}{2}}\right), \quad \varepsilon+0 .
\end{array}\right.
$$

An estimate in $\mathrm{L}_{2}$-norm for the derivatives $z_{x}$ and $z_{t}$ is furnished by formula (26a). We obtain

$$
\int_{0}^{\ell-\delta}\left(z_{x}^{2}+z_{t}^{2}\right) d x=0\left(\varepsilon^{\frac{1}{2}}\right), \quad \varepsilon+0
$$

uniformly valid for $0 \leq t \leq T$.

REMARK. Note that the right-hand side function of the differential equation (57) does not satisfy the continuity condition $(3, i)$ of theorem 1 but has instead finite jump discontinuities. One easily verifies that this does not affect the validity of the theorem.

It follows from the formulae (48), (49), (52) and (54) that

$$
\left\{\begin{array}{l}
\sup _{0 \leq \xi<\infty}\left|v_{2}(\xi, t ; \varepsilon)\right|=0(1), \quad \varepsilon+0 \\
0 \leq t \leq T \\
\sup _{0 \leq x \leq \ell}\left|v_{3}(x, \tau ; \varepsilon)\right|=0(1), \quad \varepsilon+0 \\
0 \leq \tau<\infty \\
\sup _{0 \leq t \leq T}\left|\Theta \Phi\left(t-t_{0} ; \varepsilon\right)\right|=0\left(\varepsilon^{\frac{1}{2}}\right), \\
\end{array}\right.
$$

Hence we obtain from (42), (50), (56) and (61)

(63) $u(x, t ; \varepsilon)=\tilde{w}(x, t ; \varepsilon)+\left\{k_{2}(t)-\tilde{w}(l, t ; \varepsilon)\right\} \exp \left[\frac{b(l, t)}{c^{2}(\ell, t)} \cdot \frac{x-\ell}{\varepsilon}\right]+0\left(\varepsilon^{\frac{1}{4}}\right), \quad \varepsilon \downarrow 0$ uniformly valid in any compact subset $\Omega \subset \overline{\mathrm{D}}$, and also

$$
u(x, t ; \varepsilon)=\tilde{w}(x, t ; \varepsilon)+O\left(\varepsilon^{\frac{1}{2}}\right), \quad \varepsilon \downarrow 0
$$

uniformly valid in any compact subset $\Omega \subset\{(x, t) \mid 0 \leq x \leq \ell-\delta, 0 \leq t<\infty\}$ with $\delta$ an arbitrarily small positive constant independent of $\varepsilon$ and satisfying $0<\delta<\ell$. 
Putting

$$
z(x, t ; \varepsilon)=w(x, t)-\tilde{w}(x, t ; \varepsilon)
$$

we obtain from (30) and (41)

(66)

$$
\left\{\begin{array}{l}
a(x, t) \frac{\partial z}{\partial t}+b(x, t) \frac{\partial z}{\partial x}+d(x, t) z=0, \quad(x, t) \in D \\
z(x, 0 ; \varepsilon)=0, \quad 0 \leq x \leq l \\
z(0, t ; \varepsilon)=c t \exp \left(-\frac{t}{\sqrt{\varepsilon}}\right), \quad 0 \leq t<\infty .
\end{array}\right.
$$

Multiplying this differential equation by $2 \mathrm{Z}$, integrating along characteristics and applying GRONWALL's lemma we get

$$
\mathrm{z}(\mathrm{x}, \mathrm{t} ; \varepsilon)=0\left(\varepsilon^{\frac{1}{2}}\right), \quad \varepsilon+0
$$

uniformly valid in any compact subset $\Omega \subset \overline{\mathrm{D}}$.

Hence we conclude from (65) and (67) that the formulae (63) and (64) remain valid if we replace $\tilde{w}$ by $w$.

From (62) it follows that

(68)

$$
\begin{cases}\int_{0}^{l-\delta}\left(u_{x}-\tilde{u}_{x}\right)^{2} d x=0\left(\varepsilon^{\frac{1}{2}}\right), & \varepsilon+0 \\ \int_{0}^{l-\delta}\left(u_{t}-\tilde{u}_{t}\right)^{2} d x=0\left(\varepsilon^{\frac{1}{2}}\right), & \varepsilon+0\end{cases}
$$

uniformly valid for $0 \leq t \leq T$. Evaluating $\tilde{u}_{x}$ and $\tilde{u}_{t}$ from (42) and using the formulae

$$
\begin{cases}\int_{0}^{\ell}\left(w_{x}-\tilde{w}_{x}\right)^{2} d x=\int_{0}^{l} z_{x}^{2} d x=0\left(\varepsilon^{\frac{1}{2}}\right), & \varepsilon+0 \\ \int_{0}^{l}\left(w_{t}-\tilde{w}_{t}\right)^{2} d x=\int_{0}^{l} z_{t}^{2} d x=0\left(\varepsilon^{\frac{1}{2}}\right), & \varepsilon+0\end{cases}
$$


uniformly valid for $0 \leq t \leq T$ (these formulae can be established by writing the solution $Z$ of (66) in explicit form, cf. (37), (38) and (39)) we obtain from (68) after a straightforward calculation the estimates

$$
\int_{0}^{\ell-\delta}\left[\frac{\partial u}{\partial x}(x, t ; \varepsilon)-\frac{\partial w}{\partial x}(x, t)\right]^{2} d x=0\left(\varepsilon^{\frac{1}{2}}\right), \quad \varepsilon+0
$$

and

$$
\int_{0}^{\ell-\delta}\left[\frac{\partial u}{\partial t}(x, t ; \varepsilon)-\frac{\partial w}{\partial t}(x, t)+\left\{\frac{\partial w}{\partial t}(x, 0)-h(x)\right\} \exp \left[-a(x, 0) \frac{t}{\varepsilon}\right]\right]^{2} d x=0\left(\varepsilon^{\frac{1}{2}}\right), \quad \varepsilon+0
$$

uniformly valid for $0 \leq t \leq T$.

We summarize the results in

THEOREM 2. Let $u(x, t ; \varepsilon)$ be the solution of the initial-boundary value problem (28) where all data are $C^{\infty}$-functions which in addition satisfy (29).

Then we have

$$
u(x, t ; \varepsilon)=w(x, t)+0\left(\varepsilon^{\frac{1}{2}}\right), \quad \varepsilon+0
$$

uniformly valid in any compact subset $\Omega \subset\{(\mathrm{x}, \mathrm{t}) \mid 0 \leq \mathrm{x} \leq \ell-\delta, 0 \leq \mathrm{t}<\infty\}$ where $\mathrm{w}(\mathrm{x}, \mathrm{t})$ is the solution of the reduced problem $(30)$ and $\delta$ is an arbitrarily small positive constant independent of $\varepsilon$ and satisfying $0<\delta<\ell$. In addition we have

$$
u(x, t ; \varepsilon)=w(x, t)+\left\{k_{2}(t)-w(l, t)\right\} \exp \left[\frac{b(l, t)}{c^{2}(l, t)} \cdot \frac{x-\ell}{\varepsilon}\right]+0\left(\varepsilon^{\frac{1}{4}}\right), \quad \varepsilon+0
$$

uniformly valid in any compact subset $\Omega \subset \overline{\mathrm{D}}$, and also

$$
\int_{0}^{\ell-\delta}\left[\frac{\partial u}{\partial x}(x, t ; \varepsilon)-\frac{\partial w}{\partial x}(x, t)\right]^{2} d x=0\left(\varepsilon^{\frac{1}{2}}\right), \quad \varepsilon \downarrow 0
$$

and

$$
\int_{0}^{\ell-\delta}\left[\frac{\partial u}{\partial t}(x, t ; \varepsilon)-\frac{\partial w}{\partial t}(x, t)+\left\{\frac{\partial w}{\partial t}(x, 0)-h(x)\right\} \exp \left[-a(x, 0) \frac{t}{\varepsilon}\right]\right]^{2} d x=0\left(\varepsilon^{\frac{1}{2}}\right), \quad \varepsilon+0
$$

uniformly valid for $0 \leq t \leq \mathrm{T}$. 
REMARK. The statements of theorem 2 remain valid if the condition (29b) is replaced by

$$
|b(x, t)|<a(x, t) c(x, t) \text { in } \bar{D}
$$

provided that the following requirements are fulfilled

(i) $b(\ell, t)>0$ for $t \geq 0$ (this condition implies that there is a boundary layer at $\mathrm{x}=l, \mathrm{t} \geq 0$ )

(ii) $b(0, t) \neq 0$ for $t \geq 0$

(iii) the subcharacteristic through the origin has a positive slope in $(0,0)$, so $\mathrm{a}(0,0) / \mathrm{b}(0,0)>0$

(iv) through each point in $\bar{D}$ there passes exactly one subcharacteristic and this subcharacteristic has exactly one point in common with the part of the boundary given by $\{(x, t) \mid x=0, t \geq 0\} \cup\{(x, t) \mid t=0,0 \leq x \leq \ell\}$

(compare these requirements with the conditions (ii) to (iv) mentioned in the remark following the proof of lemma 1). It follows from the timelike condition (69) and the requirements (i) to (iv) that necessarily

$$
b(0, t)>0 \text { for } t \geq 0
$$


CHARACTERISTIC BOUNDARY VALUE PROBLEMS FOR LINEAR HYPERBOLIC DIFFERENTIAL EQUATIONS

In this chapter we shall discuss the characteristic boundary value problem (singular perturbation problem)

$$
\left\{\begin{array}{l}
\varepsilon L_{2}[u]+L_{1}^{(\varepsilon)}[u]=f(x, y ; \varepsilon), \quad(x, y) \in D \\
u(x, 0 ; \varepsilon)=g(x ; \varepsilon), \quad 0 \leq x<\infty \\
u(\phi(y), y ; \varepsilon)=h(y ; \varepsilon), \quad 0 \leq y<\infty
\end{array}\right.
$$

where $\varepsilon$ is a small positive parameter, i.e. $0<\varepsilon<1$, and the differential operators $\mathrm{L}_{2}$ and $\mathrm{L}_{1}^{(\varepsilon)}$ are defined by

$$
\left\{\begin{array}{l}
L_{2}[u]=\frac{\partial^{2} u}{\partial x \partial y} \\
L_{1}^{(\varepsilon)}[u]=a(x, y ; \varepsilon) \frac{\partial u}{\partial x}+b(x, y ; \varepsilon) \frac{\partial u}{\partial y}+c(x, y ; \varepsilon) u
\end{array}\right.
$$

The functions $a, b, c, f, g$ and $h$ depend on the variables $x$ and/or $y$ and on the parameter $\varepsilon$.

The region $D$ is defined by

$$
\mathrm{D}=\{(\mathrm{x}, \mathrm{y}) \mid \phi(\mathrm{y})<\mathrm{x}<\infty, 0<\mathrm{y}<\infty\}
$$

where $\phi(y)$ is some non-decreasing differentiable function defined for $0 \leq y<\infty$ and satisfying $\phi(0)=0$. Note that the part of the boundary $\partial D$ given by $y=0, x \geq 0$ is characteristic, whereas the part given by $x=\phi(y)$, $\mathrm{y} \geq 0$ is timelike or characteristic.

It will be assumed that the boundary functions $g$ and $h$ are continuous; we require in particular 


$$
g(0 ; \varepsilon)=h(0 ; \varepsilon)
$$

to be valid for all $\varepsilon$ with $0<\varepsilon<1$.

In addition we shall assume the functions $a$ and $b$ to be strictly positive. This condition can be shown to be equivalent to the growth conditions that were discussed in section 1 of chapter III.

In section 1 we shall derive by means of the energy integral method a priori estimates for the solution $u(x, y ; \varepsilon)$ of problem (1).

In the sections 2 and 3 we shall construct an approximation for the solution $u$ of a problem of the type (1).

\section{A priori estimates}

We consider the characteristic boundary value problem (1) under the following conditions

(2)

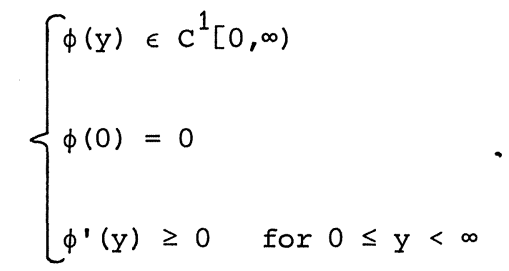

for each compact subset $\Omega \subset \bar{D}$ there exists a positive constant $\varepsilon_{1}$ (depending on $\Omega$ ) such that

(i) $\quad a(x, y ; \varepsilon) \in C^{1}(\Omega), \quad b(x, y ; \varepsilon) \in C^{1}(\Omega)$

$c(x, y ; \varepsilon) \in C^{0}(\Omega), \quad f(x, y ; \varepsilon) \in C^{0}(\Omega)$

(3) valid for every value of $\varepsilon$ satisfying $0<\varepsilon \leq \varepsilon_{1}$

(ii) the functions $a(x, y ; \varepsilon), b(x, y ; \varepsilon)$ and their first derivatives, as well as the function $c(x, y ; \varepsilon)$, are uniformly bounded in $\Omega$ for $0<\varepsilon \leq \varepsilon_{1}$

(iii) $a(x, y ; \varepsilon) \geq p>0, b(x, y ; \varepsilon) \geq p>0$ valid for all $(x, y) \in \Omega$ and all $\varepsilon \in\left(0, \varepsilon_{1}\right]$, where $p$ is some constant depending on $\Omega$ and $\varepsilon_{1}$ but independent of $\varepsilon$ 


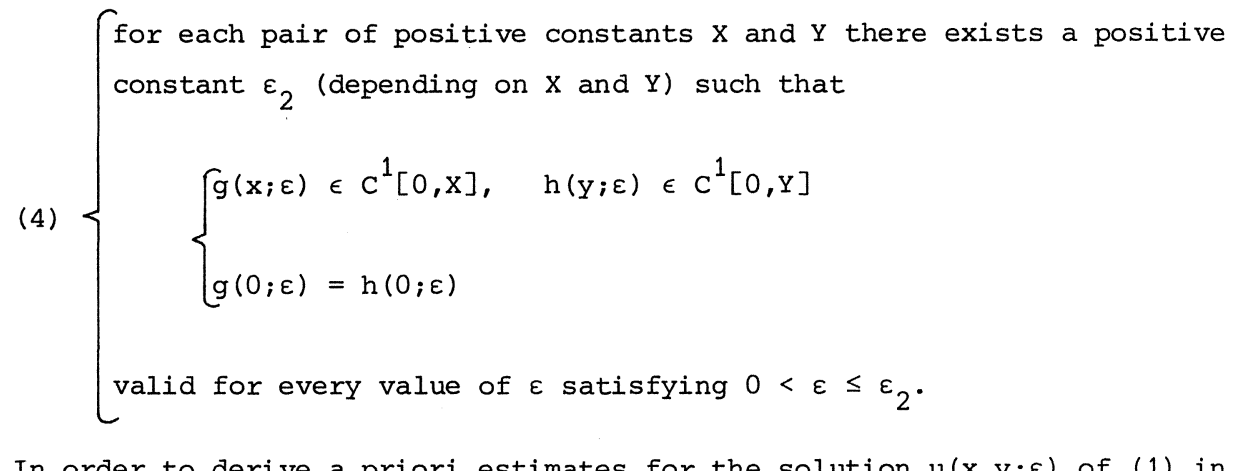
some compact subset $S \subset \bar{D}$ we enclose $S$ by a region

$$
\Omega=\left\{(\mathrm{x}, \mathrm{y}) \mid \phi(\mathrm{y}) \leq \mathrm{x} \leq \mathrm{S}_{\mathrm{x}}+\lambda\left(\mathrm{S}_{\mathrm{y}}-\mathrm{y}\right), \mathrm{y} \geq 0\right\}
$$

where

$$
S_{x}=\sup _{(x, y) \in S} x, \quad S_{y}=\sup _{(x, y) \in S} y
$$

and $\lambda$ is some positive constant, independent of $\varepsilon$, to be determined later on (see figure 1).

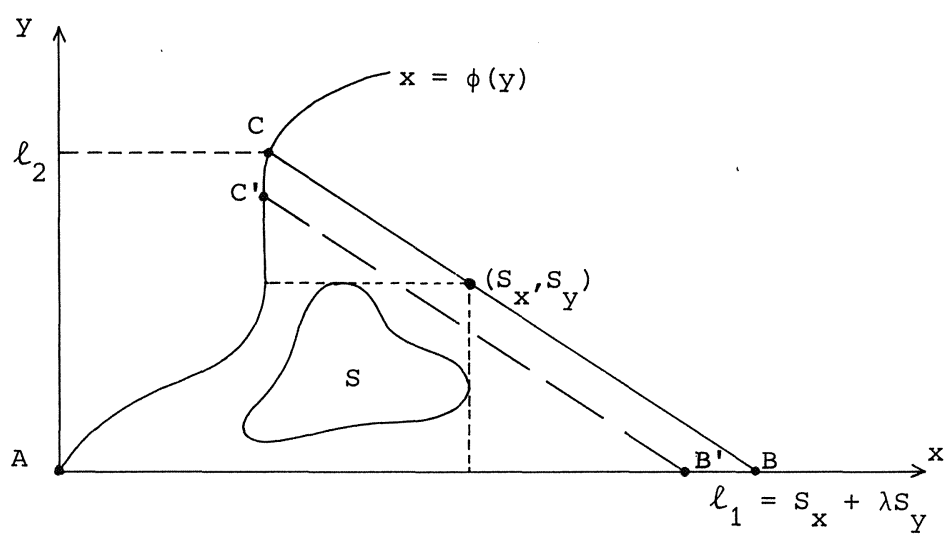

\section{Figure 1: The region $\Omega$}

We define the constant $\ell_{1}$ by $\ell_{1}=s_{x}+\lambda s_{y}$ and we shall denote the $y$-coordinate of the point $\mathrm{c}$ by $\ell_{2}$. 
Let $\alpha=\alpha(x, y ; \varepsilon), \beta=\beta(x, y ; \varepsilon)$ and $\gamma=\gamma(x, y ; \varepsilon)$ be continuously differentiable functions (depending on the variables $x$ and $y$ and on the parameter $\varepsilon$ ) to be specified later on.

We multiply the differential equation (1) by $2 \alpha u$ to obtain

$$
\frac{\partial}{\partial y}\left(\alpha b u^{2}+\varepsilon \alpha u u_{x}\right)+\frac{\partial}{\partial x}\left(\alpha a u^{2}+\varepsilon \alpha u u_{y}\right)=
$$

(6)

$$
\begin{aligned}
& \left\{\frac{\partial(\alpha a)}{\partial x}+\frac{\partial(\alpha b)}{\partial y}-2 \alpha c\right\} u^{2}+\varepsilon \frac{\partial \alpha}{\partial y} u_{x}+\varepsilon \frac{\partial \alpha}{\partial x} u_{y} \\
& +2 \varepsilon \alpha u_{x} u_{y}+2 \alpha f u
\end{aligned}
$$

valid for $(\mathrm{x}, \mathrm{y}) \in \Omega$ and $0<\varepsilon \leq \varepsilon_{0}=\min \left(\varepsilon_{1}, \varepsilon_{2}\right)$, the values of $\varepsilon_{1}$ and $\varepsilon_{2}$ being determined by the region $\Omega$ (see the conditions $(3, i)$ and $(4)$ ).

Multiplication of (1) by $2 \beta u_{x}$ yields

$$
\frac{\partial}{\partial y}\left(\varepsilon \beta u_{x}^{2}\right)=\varepsilon \frac{\partial \beta}{\partial y} u_{x}^{2}-2 \beta c u u_{x}
$$

$$
-2 \beta a u_{x}^{2}-2 \beta b u_{x} u_{y}+2 \beta f u_{x}
$$

valid for $(x, y) \in \Omega$ and $0<\varepsilon \leq \varepsilon_{0}$.

Similarly, multiplication by $2 \gamma u_{y}$ leads to

$$
\frac{\partial}{\partial x}\left(\varepsilon \gamma u_{y}^{2}\right)=\varepsilon \frac{\partial \gamma}{\partial x} u_{y}^{2}-2 \gamma c u u_{y}
$$

$$
-2 \gamma b u_{y}^{2}-2 \gamma a u_{x} u_{y}+2 \gamma f u_{y}
$$

likewise valid for $(\mathrm{x}, \mathrm{y}) \in \Omega$ and $0<\varepsilon \leq \varepsilon_{0}$.

Adding (6), (7) and (8) we obtain

$$
\frac{\partial}{\partial y} Q_{1}+\frac{\partial}{\partial x} Q_{2}=Q_{3}+Q_{4}
$$

valid for $(x, y) \in \Omega$ and $0<\varepsilon \leq \varepsilon_{0}$, where the expressions $Q_{i}(i=1,2,3,4)$ are given by 
$(10)\left\{\begin{aligned} Q_{1}= & \alpha b u^{2}+\varepsilon \alpha u u_{x}+\varepsilon \beta u_{x}^{2} \\ Q_{2}= & \alpha a u^{2}+\varepsilon \alpha u_{y}+\varepsilon \gamma u_{y}^{2} \\ Q_{3}= & \left\{\frac{\partial(\alpha a)}{\partial x}+\frac{\partial(\alpha b)}{\partial y}-2 \alpha c\right\} u^{2}+\varepsilon \frac{\partial \alpha}{\partial y} u_{x}+\varepsilon \frac{\partial \alpha}{\partial x} u_{y} \\ & +\varepsilon \frac{\partial \beta}{\partial y} u_{x}^{2}+\varepsilon \frac{\partial \gamma}{\partial x} u_{y}^{2} \\ Q_{4}= & -2 \beta a u_{x}^{2}-2(\beta b+\gamma a-\varepsilon \alpha) u_{x} u_{y}-2 \gamma b u_{y}^{2} \\ & +2 \alpha f u+2(f-c u)\left(\beta u_{x}+\gamma u_{y}\right) .\end{aligned}\right.$

Integrating (9) over the region $\Omega$ and using GREEN's theorem in the plane we obtain (see figure 1)

$$
\int_{B}^{C}\left(Q_{2}+\lambda Q_{1}\right) d y=\int_{A}^{B} Q_{1} d x+\int_{A}^{C}\left(Q_{2}-\phi^{\prime} Q_{1}\right) d y+\iint_{\Omega}\left(Q_{3}+Q_{4}\right) d x d y .
$$

We shall now specify the functions $\alpha, \beta$ and $\gamma$.

Let the variable $\rho$ be defined by

$$
\rho=x-\phi(y)
$$

and let $\delta$ be an arbitrarily small positive number which does not depend on $\varepsilon$. Let $\Psi(\rho)$ be some $C^{\infty}$-function defined for $\rho \geq 0$ which satisfies

$$
\left\{\begin{array}{l}
\Psi(\rho)= \begin{cases}1 & \text { for } \rho \geq \delta \\
0 & \text { for } 0 \leq \rho \leq \frac{1}{2} \delta\end{cases} \\
\Psi(\rho) \text { monotonically increasing for } \frac{1}{2} \delta \leq \rho \leq \delta
\end{array}\right.
$$

(see figure $2 a$ ) and let the $C^{\infty}$-function $\Phi(\rho ; \varepsilon)$ be defined by

$$
\Phi(\rho ; \varepsilon)=\varepsilon+(1-\varepsilon) \Psi(\rho), \quad \rho \geq 0
$$

(see figure $2 \mathrm{~b}$ ). 


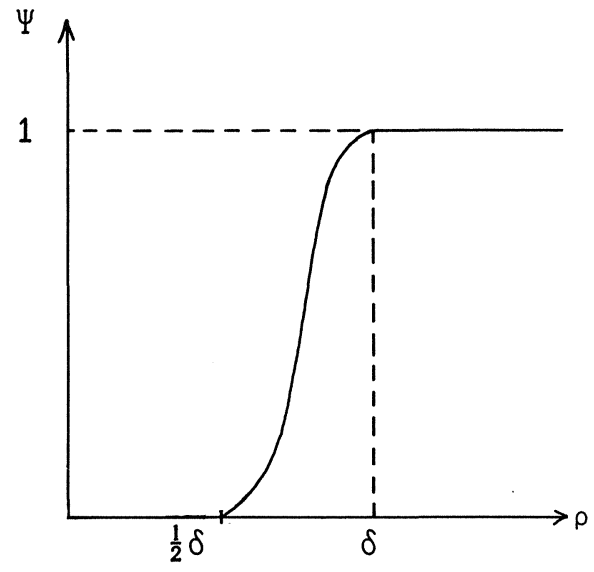

Figure 2a

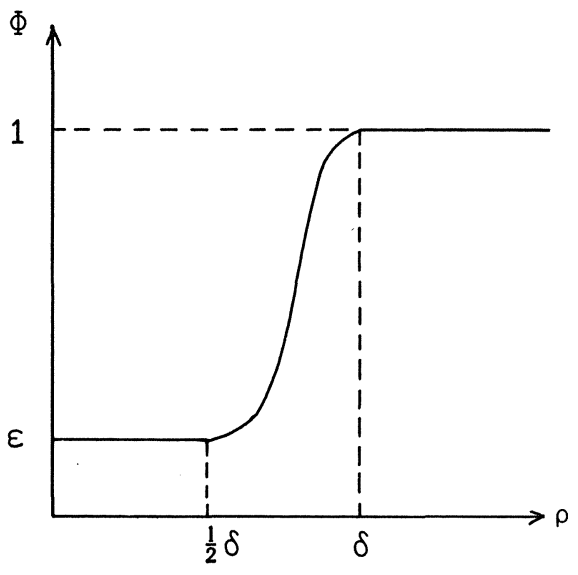

Figure 2b

We now choose the functions $\alpha, \beta$ and $\gamma$ as follows

(13) $\left\{\begin{array}{l}\alpha=\alpha(x, y ; \varepsilon)=a(x, y ; \varepsilon)+\lambda b(x, y ; \varepsilon)=a+\lambda b \\ \beta=\beta(x, y ; \varepsilon)=\mu a(x, y ; \varepsilon) \Phi(\rho ; \varepsilon)+\varepsilon \lambda=\mu a \Phi+\varepsilon \lambda \\ \gamma=\gamma(x, y ; \varepsilon)=\mu b(x, y ; \varepsilon) \Phi(\rho ; \varepsilon)+\varepsilon=\mu b \Phi+\varepsilon\end{array}\right.$

where $\mu$ is some positive constant, independent of $\varepsilon$, to be determined later on (this leaves $\lambda$ and $\mu$ to be specified).

Now that we have chosen $\alpha, \beta$ and $\gamma$ we shall have a closer look at the integrands in (11).

Since we have

$$
u_{y}=\frac{d u}{d y}-\phi^{\prime} u_{x} \quad \text { on } A C
$$

we obtain along $\mathrm{AC}$, using (10)

$$
\begin{aligned}
Q_{2}-\phi^{\prime} Q_{1}= & -\varepsilon \phi^{\prime}\left(\beta-\gamma \phi^{\prime}\right) u_{x}^{2}-2 \varepsilon \alpha \phi^{\prime} u_{x}-2 \varepsilon \gamma \phi^{\prime} \frac{d u}{d y} u_{x} \\
& +\alpha\left(a-b \phi^{\prime}\right) u^{2}+\varepsilon \alpha u \frac{d u}{d y}+\varepsilon \gamma\left(\frac{d u}{d y}\right)^{2}
\end{aligned}
$$


This expression contains the function $u_{x}$ that we do not know a priori along AC. However, it is possible to eliminate $u_{x}$ by an appropriate choice of $\lambda$ and $\mu$. Let us assume for the moment that $\lambda$ and $\mu$ have been chosen such that

$$
\beta-\gamma \phi^{\prime} \geq \varepsilon \quad \text { on } A C \text {. }
$$

Then it follows from (14) that there exists a positive constant $M_{1}$ (depending on $\Omega, \lambda$ and $\mu$ but independent of $\varepsilon$ ) such that

$$
Q_{2}-\phi^{\prime} Q_{1} \leq M_{1}\left\{u^{2}+\varepsilon^{2}\left(\frac{d u}{d y}\right)^{2}\right\} \quad \text { on } A C .
$$

What conditions have to be imposed on $\lambda$ and $\mu$ in order that (15) holds? Using (13) we see that (15) is fulfilled iff

$$
\lambda-\phi^{\prime} \geq \mu\left(b \phi^{\prime}-a\right)+1 \quad \text { on } A C .
$$

We now choose $\lambda$ as follows:

$$
\lambda=2+\max _{0 \leq \mathrm{y} \leq \ell_{2}} \phi^{\prime}(\mathrm{y}) .
$$

Then it follows that (17) is satisfied if $\mu$ is chosen such that

$$
0<\mu<\mu_{1}=\frac{1}{1+\theta}
$$

where the positive constant $\theta$, independent of $\varepsilon$, is given by

$$
\Theta=\sup _{\substack{0 \leq y \leq \ell_{2} \\ 0<\varepsilon \leq \varepsilon_{0}}}\left\{a(\phi(y), y ; \varepsilon)+b(\phi(y), y ; \varepsilon) \phi^{\prime}(y)\right\}
$$

As for the expression $Q_{3}$, it is easily established that there exist positive constants $\mathrm{M}_{2}$ (depending on $\Omega$ but independent of $\mu$ and $\varepsilon$ ) and $\mathrm{M}_{3}$ (depending on $\Omega$ but independent of $\mu$ and $\varepsilon$ ) such that

$$
Q_{3} \leq M_{2}\left(u^{2}+\varepsilon^{2} u_{x}^{2}+\varepsilon^{2} u_{y}^{2}\right)+\varepsilon \mu M_{3}\left(u_{x}^{2}+u_{y}^{2}\right)
$$

valid for $(\mathrm{x}, \mathrm{y}) \in \Omega$ and $0<\varepsilon \leq \varepsilon_{0}$.

Next we consider $Q_{4}$. Substituting (13) into (10) we obtain 


$$
\begin{aligned}
Q_{4}= & -2 \mu \Phi\left\{\left(a u_{x}+b u_{y}\right)^{2}+(c u-f)\left(a u_{x}+b u_{y}\right)\right\} \\
& +2(a+\lambda b) f u+2 \varepsilon(f-c u)\left(\lambda u_{x}+u_{y}\right) \\
& -2 \varepsilon\left(\lambda a u_{x}^{2}+b u_{y}^{2}\right) .
\end{aligned}
$$

Using $(3, i i i)$ it is not difficult to verify that there exist positive constants $M_{4}$ (depending on $\Omega$ and $\mu$ but independent of $\varepsilon$ ) and $M_{5}$ (depending on $\Omega$ but independent of $\mu$ and $\varepsilon$ ) such that

$$
Q_{4} \leq M_{4}\left(u^{2}+\varepsilon^{2} u_{x}^{2}+\varepsilon^{2} u_{y}^{2}+f^{2}\right)-\varepsilon M_{5}\left(u_{x}^{2}+u_{y}^{2}\right)
$$

valid for $(\mathrm{x}, \mathrm{y}) \in \Omega$ and $0<\varepsilon \leq \varepsilon_{0}$.

For $\mu$ sufficiently small, viz.

$$
0<\mu<\mu_{0}=\min \left(\mu_{1}, \frac{M_{5}}{M_{3}}\right)
$$

it follows from (18) and (19) that

$$
Q_{3}+Q_{4} \leq\left(M_{2}+M_{4}\right)\left(u^{2}+\varepsilon^{2} u_{x}^{2}+\varepsilon^{2} u_{y}^{2}+f^{2}\right)
$$

$$
\leq\left(M_{2}+M_{4}\right)\left(u^{2}+\varepsilon \Phi u_{x}^{2}+\varepsilon \Phi u_{y}^{2}+f^{2}\right)
$$

valid for $(\mathrm{x}, \mathrm{y}) \in \Omega$ and $0<\varepsilon \leq \varepsilon_{0}$. From now on it will be assumed that the value of $\mu$ is fixed and satisfies (20).

Finally, we consider the expression $Q_{2}+\lambda Q_{1}$. It follows from (10) and (13) that on $\mathrm{BC}$

$$
\begin{aligned}
Q_{2}+\lambda Q_{1}= & (a+\lambda b)^{2} u^{2}+\varepsilon(a+\lambda b) u\left(\lambda u_{x}+u_{y}\right)+\varepsilon^{2} \lambda^{2} u_{x}^{2}+\varepsilon^{2} u_{y}^{2} \\
& +\varepsilon \lambda \mu a \Phi u_{x}^{2}+\varepsilon \mu b \Phi u_{y}^{2} \\
= & \left\{\frac{1}{2}(a+\lambda b) u+\varepsilon \lambda u_{x}\right\}^{2}+\left\{\frac{1}{2}(a+\lambda b) u+\varepsilon u_{y}\right\}^{2} \\
& +\frac{1}{2}(a+\lambda b)^{2} u^{2}+\varepsilon \lambda \mu a \Phi u_{x}^{2}+\varepsilon \mu b \Phi u_{y}^{2} \\
\geq & m\left(u^{2}+\varepsilon \Phi u_{x}^{2}+\varepsilon \Phi u_{y}^{2}\right)
\end{aligned}
$$


where $\mathrm{m}$ is some positive constant depending on $\Omega$ but independent of $\varepsilon$.

Since it is easily established that there exists a positive constant $M_{6}$ (depending on $\Omega$ but independent of $\varepsilon$ ) such that

$$
Q_{1} \leq M_{6}\left(u^{2}+\varepsilon u_{x}^{2}\right)
$$

valid for $(x, y) \in \Omega$ and $0<\varepsilon \leq \varepsilon_{0}$, we obtain from (11), (16), (21), (22) and (23)

$$
m \int_{B}^{C}\left(u^{2}+\varepsilon \Phi u_{x}^{2}+\varepsilon \Phi u_{y}^{2}\right) d y \leq M \iint_{\Omega}\left(u^{2}+\varepsilon \Phi u_{x}^{2}+\varepsilon \Phi u_{y}^{2}\right) d x d y+M k^{2}(\Omega, \varepsilon)
$$

valid for $0<\varepsilon \leq \varepsilon_{0}$, where the positive constant $M$, depending on $\Omega$ but independent of $\varepsilon$, is given by

$$
M=\max \left(M_{1}, M_{2}+M_{4}, M_{6}\right)
$$

and the positive constant $\mathrm{K}(\Omega, \varepsilon)$, depending on $\Omega$ and $\varepsilon$, is defined by

$$
\mathrm{K}(\Omega, \varepsilon)=\|f\|_{\Omega}+\|\mathrm{g}\|_{\left[0, \ell_{1}\right]}+\|\mathrm{h}\|_{\left[0, \ell_{2}\right]}+\varepsilon^{\frac{1}{2}}\left\|_{\mathrm{x}}\right\|_{\left[0, \ell_{1}\right]}+\varepsilon\left\|_{\mathrm{y}}\right\|_{\left[0, \ell_{2}\right]} \cdot
$$

It is easily verified that (24) with the same values of $\mathrm{m}$ and $M$ is also valid for every region $\Omega^{\prime}=A^{\prime} C^{\prime}$ inside $\Omega=A B C$ with $B^{\prime} C^{\prime} / / B C$ (see figure 1). Hence we obtain

$$
\int_{B^{\prime}}^{C^{\prime}}\left(u^{2}+\varepsilon \Phi u_{x}^{2}+\varepsilon \Phi u_{y}^{2}\right) d y-\frac{M}{m} \iint_{\Omega^{\prime}}\left(u^{2}+\varepsilon \Phi u_{x}^{2}+\varepsilon \Phi u_{y}^{2}\right) d x d y \leq \frac{M}{m} k^{2}(\Omega, \varepsilon)
$$

valid for $0<\varepsilon \leq \varepsilon_{0}$ and every $\Omega^{\prime} \subset \Omega$ with $B^{\prime} C^{\prime} / / B C$.

Introducing new variables $\xi$ and $n$ by

(27) $\left\{\begin{array}{l}\xi=x+\lambda y \\ \eta=y-\lambda x\end{array}\right.$

we transform the region $\Omega$ into a region $\Omega^{*}$ (see figure 3 ). 

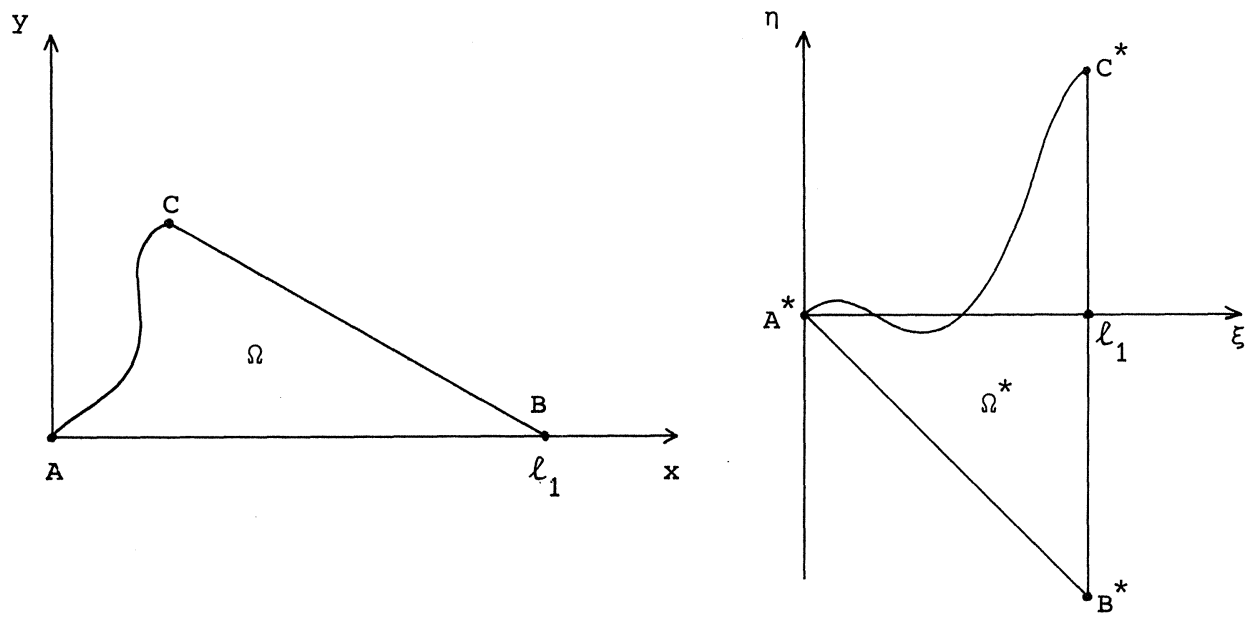

Figure 3

The transformed boundaries $A^{*} B^{*}, B^{*} C^{*}$ and $A^{*} C^{*}$ are described by

$$
\left\{\begin{array}{l}
A^{*}{ }^{*}=\left\{(\xi, \eta) \mid 0 \leq \xi \leq \ell_{1}, n=-\lambda \xi\right\} \\
B^{*} C^{*}=\left\{(\xi, \eta) \mid \xi=\ell_{1},-\lambda \ell_{1} \leq n \leq-\lambda \ell_{1}+\left(1+\lambda^{2}\right) \ell_{2}\right\} \\
A^{*} C^{*}=\left\{(\xi, n) \mid 0 \leq \xi \leq \ell_{1}, n=F(\xi)\right\}
\end{array}\right.
$$

where

$$
F(\xi)=-\lambda \xi+\left(1+\lambda^{2}\right) \psi^{-1}(\xi)
$$

with $\psi^{-1}$ the inverse of the monotonically increasing function

$$
\psi(y)=\phi(y)+\lambda y, \quad y \geq 0
$$

We have

$$
u_{x}^{2}+u_{y}^{2}=\left(u_{\xi}-\lambda u_{\eta}\right)^{2}+\left(\lambda u_{\xi}+u_{n}\right)^{2}=\left(1+\lambda^{2}\right)\left(u_{\xi}^{2}+u_{n}^{2}\right) .
$$

Using this result we get from (26) 


$$
\int_{-\lambda z}^{F(z)} I(z, \eta ; \varepsilon) d \eta-\frac{M}{m} \int_{0}^{z}\left\{\int_{-\lambda \xi}^{F(\xi)} I(\xi, \eta ; \varepsilon) d \eta\right\} d \xi \leq\left(1+\lambda^{2}\right) \frac{M}{m} k^{2}(\Omega, \varepsilon)
$$

valid for every $z \in\left[0, \ell_{1}\right]$ and $0<\varepsilon \leq \varepsilon_{0}$, where

$$
I(\xi, \eta ; \varepsilon)=u^{2}+\varepsilon\left(1+\lambda^{2}\right) \Phi\left(u_{\xi}^{2}+u_{\eta}^{2}\right) .
$$

Applying GRONWALL's lemma we obtain

$$
\begin{aligned}
\int_{-\lambda \xi}^{F(\xi)}\left(u^{2}+\varepsilon \Phi u_{\xi}^{2}+\varepsilon \Phi u_{n}^{2}\right) d \eta & \leq\left(1+\lambda^{2}\right) \frac{M}{m} K^{2}(\Omega, \varepsilon) \exp \left(\frac{M}{m} \xi\right) \\
& \leq C(\Omega) K^{2}(\Omega, \varepsilon)
\end{aligned}
$$

valid for $0 \leq \xi \leq \ell_{1}$ and $0<\varepsilon \leq \varepsilon_{0}$, the positive constant $\mathrm{C}(\Omega)$ (depending on $\Omega$ but independent of $\varepsilon$ ) being given by

$$
C(\Omega)=\left(1+\lambda^{2}\right) \frac{M}{m} \exp \left(\frac{M l}{m}\right) \text {. }
$$

Since the transformation (27) transforms, the part of the curve $x=\phi(y)+\delta$ inside $\Omega$ into the curve $\eta=F(\xi-\delta)-\lambda \delta, \delta \leq \xi \leq \ell_{1}$ inside $\Omega^{*}$ it follows that

$$
\Phi \equiv 1 \text { for }-\lambda \xi \leq \eta \leq G(\xi), \delta \leq \xi \leq \ell_{1}
$$

where $G(\xi)=F(\xi-\delta)-\lambda \delta$. Hence we obtain from (28) and (29)

(30a) $\begin{cases}\int_{-\lambda \xi}^{F(\xi)}\left(u^{2}+\varepsilon^{2} u_{\xi}^{2}+\varepsilon^{2} u_{\eta}^{2}\right) d \eta \leq c(\Omega) k^{2}(\Omega, \varepsilon) & \text { for } 0 \leq \xi \leq \ell_{1} \\ \int_{-\lambda \xi}^{G(\xi)}\left(u^{2}+\varepsilon u_{\xi}^{2}+\varepsilon u_{\eta}^{2}\right) d \eta \leq c(\Omega) k^{2}(\Omega, \varepsilon) & \text { for } \delta \leq \xi \leq l_{1}\end{cases}$ valid for $0<\varepsilon \leq \varepsilon_{0}$.

Now that we have established estimates in $L_{2}$-norm it is not difficult to derive pointwise estimates. Using (30a) we obtain for $0 \leq \xi \leq \ell_{1},-\lambda \xi \leq \eta \leq F(\xi)$ and $0<\varepsilon \leq \varepsilon_{0}$ 


$$
\begin{aligned}
\left|u^{2}(\xi, \eta ; \varepsilon)-u^{2}(\xi,-\lambda \xi ; \varepsilon)\right| & =\left|2 \int_{-\lambda \xi}^{\eta} u(\xi, \tau ; \varepsilon) u_{\eta}(\xi, \tau ; \varepsilon) d \tau\right| \\
& \leq 2 \int_{-\lambda \xi}^{F(\xi)}\left|u(\xi, \tau ; \varepsilon) u_{\eta}(\xi, \tau ; \varepsilon)\right| d \tau \\
& \left.\leq 2\left[\int_{-\lambda \xi}^{F(\xi)} u^{2}(\xi, \tau ; \varepsilon) d \tau\right]^{\frac{1}{2}} F(\xi) \int_{-\lambda \xi}^{2} u_{\eta}^{2}(\xi, \tau ; \varepsilon) d \tau\right]^{\frac{1}{2}} \\
& \leq C(\Omega) \varepsilon^{-1 K^{2}(\Omega, \varepsilon)}
\end{aligned}
$$

with $C(\Omega)$ some generic constant depending on $\Omega$ but independent of $\varepsilon$. This yields

$$
u^{2}(\xi, \eta ; \varepsilon) \leq u^{2}(\xi,-\lambda \xi ; \varepsilon)+C(\Omega) \varepsilon^{-1} K^{2}(\Omega, \varepsilon)
$$

$$
=g^{2}(\xi ; \varepsilon)+C(\Omega) \varepsilon^{-1} K^{2}(\Omega, \varepsilon) .
$$

Using SOBOLEV's inequality for estimating $g$ in terms of $\|g\|_{\left[0, \ell_{1}\right]}$ and $\left\|g_{\mathbf{x}}\right\|\left[0, \ell_{1}\right]$ and remembering the expression (25) for $K(\Omega, \varepsilon)$ we obtain from (31)

$$
u^{2}(\xi, \eta ; \varepsilon) \leq C(\Omega) \varepsilon^{-1} K^{2}(\Omega, \varepsilon)
$$

valid for $(\xi, \eta) \in \Omega^{*}$ and $0<\varepsilon \leq \varepsilon_{0}$, and hence also

$$
|u(x, y ; \varepsilon)| \leq C(\Omega) \varepsilon^{-\frac{1}{2}} K(\Omega, \varepsilon)
$$

valid for $(x, y) \in \Omega$ and $0<\varepsilon \leq \varepsilon_{0}$, where $C(\Omega)$ is some positive constant depending on $\Omega$ but independent of $\varepsilon$.

In a similar way we obtain from (30b) the estimate

$$
u^{2}(\xi, \eta ; \varepsilon) \leq C(\Omega) \varepsilon^{-\frac{1}{2}} K^{2}(\Omega, \varepsilon)
$$

valid for $\delta \leq \xi \leq \ell_{1},-\lambda \xi \leq \eta \leq G(\xi)$ and $0<\varepsilon \leq \varepsilon_{0}$, and hence also

$$
|u(x, y ; \varepsilon)| \leq C(\Omega) \varepsilon^{-\frac{1}{4}} K(\Omega, \varepsilon)
$$


valid for $(\mathrm{x}, \mathrm{y}) \in \Omega \backslash\left\{(\mathrm{x}, \mathrm{y}) \mid \phi(\mathrm{y}) \leq \mathrm{x}<\phi(\mathrm{y})+\delta, 0 \leq \mathrm{y} \leq \ell_{2}\right\}$ and $0<\varepsilon \leq \varepsilon_{0}$, where $\mathrm{C}(\Omega)$ is some positive constant depending on $\Omega$ but independent of $\varepsilon$.

We summarize the results in

THEOREM 1. Let $\mathrm{u}(\mathrm{x}, \mathrm{y} ; \varepsilon)$ be the solution of the characteristic boundary value problem (1) under the conditions (2), (3) and (4) and let the region $\Omega \subset \bar{D}$ be defined by (5). Then we have for $\varepsilon$ sufficiently small, say $0<\varepsilon \leq \varepsilon_{0}$ with $\varepsilon_{0}$ depending on $\Omega$, the pointwise estimates

$$
\left\{\begin{array}{l}
\sup _{\Omega}|\mathrm{u}(\mathrm{x}, \mathrm{y} ; \varepsilon)| \leq \mathrm{C}(\Omega) \varepsilon^{-\frac{1}{2}} \mathrm{~K}(\Omega, \varepsilon) \\
\sup _{\widetilde{\Omega}}|\mathrm{u}(\mathrm{x}, \mathrm{y} ; \varepsilon)| \leq \mathrm{C}(\Omega) \varepsilon^{-\frac{1}{4}} \mathrm{~K}(\Omega, \varepsilon)
\end{array}\right.
$$

where the region $\tilde{\Omega}$ is defined by

$$
\tilde{\Omega}=\Omega \backslash\left\{(\mathrm{x}, \mathrm{y}) \mid \phi(\mathrm{y}) \leq \mathrm{x}<\phi(\mathrm{y})+\delta, 0 \leq \mathrm{y} \leq \ell_{2}\right\}
$$

with $\delta$ some arbitrarily small positive constant independent of $\varepsilon . \mathrm{C}(\Omega)$ is some positive constant depending on $\Omega$ but independent of $\varepsilon$, and the positive constant $\mathrm{K}(\Omega, \varepsilon)$ depending on $\Omega$ and $\varepsilon$ is given by

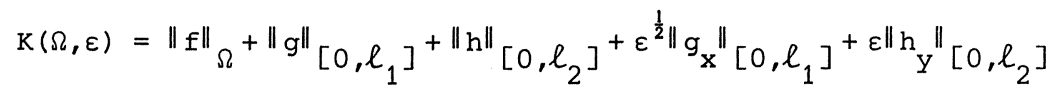

with $\|\cdot\|_{\Omega}$ denoting the $\mathrm{L}_{2}$-norm over $\Omega$, etc.

REMARKS.

1. A slight improvement (which will turn out to be of importance later on) can be obtained as follows: replacing formula (23) by the inequality

$$
Q_{1} \leq M_{6}\left(u^{2}+\varepsilon \Phi u_{x}^{2}\right)
$$

of which the validity is easily verified, it follows that the estimates (32) remain valid if the constant $\mathrm{K}(\Omega, \varepsilon)$ is replaced by $\tilde{K}(\Omega, \varepsilon)$ with

$$
\begin{aligned}
\tilde{K}(\Omega, \varepsilon)= & \|f\|_{\Omega}+\|g\|_{\left[0, \ell_{1}\right]}+\|h\|_{\left[0, \ell_{2}\right]} \\
& +\varepsilon^{\frac{1}{2} \|} \Phi^{\frac{1}{2}} g_{x}\left\|\left[0, \ell_{1}\right]+\varepsilon\right\| h_{y} \|\left[0, \ell_{2}\right] .
\end{aligned}
$$


We shall use this result in section 3 .

2. Another estimate is possible if it is given that there exists for every $\mathrm{Y}>0$ an $\varepsilon_{0}>0$ such that

$$
a(\phi(y), y ; \varepsilon)-b(\phi(y), y ; \varepsilon) \phi^{\prime}(y) \geq c_{0}>0
$$

valid for $0 \leq \mathrm{y} \leq \mathrm{Y}$ and $0<\varepsilon \leq \varepsilon_{0^{\prime}}$, where $\mathrm{c}_{0}$ is some constant depending on $Y$ and $\varepsilon_{0}$ but independent of $\varepsilon$. The condition (33) implies the absence of a boundary layer along $\mathrm{x}=\phi(y), y \geq 0$; this condition is fulfilled if for instance $\phi^{\prime}(y) \equiv 0$ for $y \geq 0$. Replacing (12) by

$$
\Psi(\rho) \equiv 1 \text { for } \rho \geq 0
$$

and adjusting the proof of theorem 1 it follows that

$$
\sup _{\Omega}|u(x, y ; \varepsilon)| \leq C(\Omega) \varepsilon^{-\frac{1}{4}} K_{1}(\Omega, \varepsilon)
$$

where $\mathrm{C}(\Omega)$ is some positive constant depending on $\Omega$ but independent of $\varepsilon$, and the positive constant $\mathrm{K}_{1}(\Omega, \varepsilon)$ depending on $\Omega$ and $\varepsilon$ is given by

$$
\begin{aligned}
\mathrm{K}_{1}(\Omega, \varepsilon)= & \|f\|_{\Omega}+\|\mathrm{g}\|\left[0, \ell_{1}\right]+\|\mathrm{h}\|\left[0, \ell_{2}\right] \\
& +\varepsilon^{\frac{1}{2}}\left\|_{\mathrm{x}}\right\|\left[0, \ell_{1}\right]+\varepsilon^{\frac{1}{2}}\left\|_{\mathrm{h}}\right\|\left[0, \ell_{2}\right]
\end{aligned}
$$

2. Approximations for the case without boundary layer along $x=\phi(y), y \geq 0$

In this section we shall construct an approximation for the solution $u(x, y ; \varepsilon)$ of the characteristic boundary value problem

$$
\left\{\begin{array}{l}
\varepsilon L_{2}[u]+L_{1}^{(\varepsilon)}[u]=f(x, y), \quad(x, y) \in D \\
u(x, 0 ; \varepsilon)=g(x), \quad 0 \leq x<\infty \\
u(\phi(y), y ; \varepsilon)=h(y), \quad 0 \leq y<\infty
\end{array}\right.
$$

where $0<\varepsilon<1$ and 


$$
\left\{\begin{array}{l}
L_{2}[u]=\frac{\partial^{2} u}{\partial x \partial y} \\
L_{1}^{(\varepsilon)}[u]=\left\{a_{1}(x, y)+\varepsilon a_{2}(x, y)\right\} \frac{\partial u}{\partial x}+\left\{b_{1}(x, y)+\varepsilon b_{2}(x, y)\right\} \frac{\partial u}{\partial y}+c(x, y) u,
\end{array}\right.
$$

the region $D$ being defined as in the previous section.

In order not to complicate the calculations it will be assumed that all data are $\mathrm{C}^{\infty}$-functions which in addition satisfy

$$
a_{1}(x, y)>0, \quad b_{1}(x, y)>0 \text { in } \bar{D}
$$

and

$$
\left\{\begin{array}{l}
\phi(0)=0 \\
\phi^{\prime}(y) \geq 0 \quad \text { for } 0 \leq y<\infty \\
g(0)=h(0) .
\end{array}\right.
$$

REMARK. Note that the differential equation (34) follows from the equation

$$
\varepsilon\left\{\frac{\partial^{2} u}{\partial t^{2}}-c^{2}(x, t) \frac{\partial^{2} u}{\partial x^{2}}\right\}+a(x, t) \frac{\partial u}{\partial t}+b(x, t) \frac{\partial u}{\partial x}+d(x, t) u=f(x, t)
$$

(with $c(x, t)>0$ ) by a transformation of coordinates. It can be shown that the conditions (35) for the equation (34) correspond to the well-known growth conditions

$$
a(x, t)>0 \text { and }|b(x, t)|<a(x, t) c(x, t)
$$

for the equation (37).

As a first approximation for the solution $u(x, y ; \varepsilon)$ of (34) we shall take the solution $\mathrm{w}(\mathrm{x}, \mathrm{y})$ of the reduced problem which is governed by the equation

$$
\mathrm{L}_{1}^{(0)}[\mathrm{w}] \equiv \mathrm{a}_{1}(\mathrm{x}, \mathrm{y}) \frac{\partial \mathrm{w}}{\partial \mathrm{x}}+\mathrm{b}_{1}(\mathrm{x}, \mathrm{y}) \frac{\partial \mathrm{w}}{\partial \mathrm{y}}+\mathrm{c}(\mathrm{x}, \mathrm{y}) \mathrm{w}=\mathrm{f}(\mathrm{x}, \mathrm{y}), \quad(\mathrm{x}, \mathrm{y}) \in \mathrm{D}
$$

The number of boundary conditions which can be imposed on solutions of this equation depends on the position of the subcharacteristics with respect to 
the part of the boundary $\partial D$ given by $x=\phi(y), y \geq 0$. If it is given that

$$
a_{1}(\phi(y), y)-b_{1}(\phi(y), y) \phi^{\prime}(y)>0 \quad \text { for } 0 \leq y<\infty
$$

then we have the situation of figure $4 \mathrm{a}$ and we can impose both boundary conditions on solutions of (38). This case will be discussed in this section. If on the other hand

$$
a_{1}(\phi(y), y)-b_{1}(\phi(y), y) \phi^{\prime}(y)<0 \quad \text { for } 0 \leq y<\infty
$$

then we have a situation as sketched in figure $4 \mathrm{~b}$ and we can impose only the boundary condition $u(x, 0 ; \varepsilon)=g(x)$ on solutions of (38). We shall discuss this problem in section 3 .

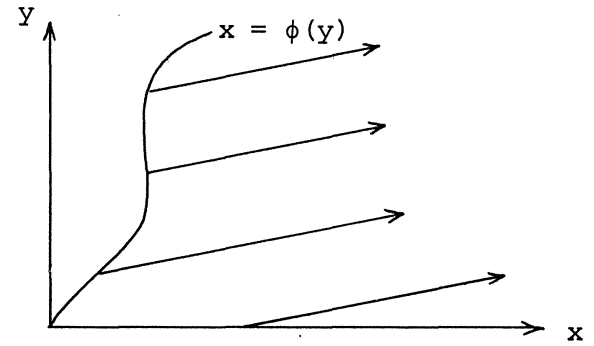

Figure $4 \mathrm{a}$

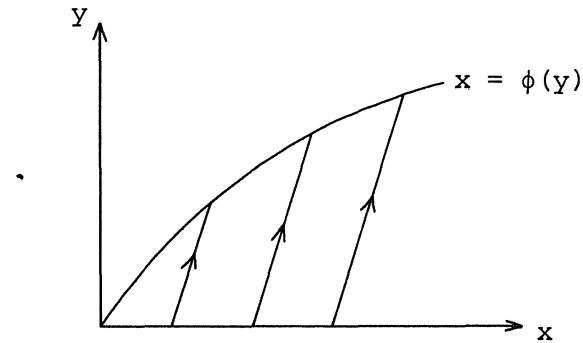

Figure $4 b$

Assuming the condition (39) to be fulfilled we take for $w$ the solution of

$$
\left\{\begin{array}{lc}
L_{1}^{(0)}[w]=f(x, y), & (x, y) \in D \\
w(x, 0)=g(x), & 0 \leq x<\infty \\
w(\phi(y), y)=h(y), & 0 \leq y<\infty .
\end{array}\right.
$$

As in section 2 of the preceding chapter we cannot evaluate $L_{2}[w]$ since the solution $w$ of (40) is not necessarily twice differentiable. This is stated in 
IEMMA 1. Let $\mathrm{u}(\mathrm{x}, \mathrm{y})$ be the solution of the boundary value problem

$$
\left\{\begin{array}{l}
a(x, y) \frac{\partial u}{\partial x}+b(x, y) \frac{\partial u}{\partial y}+c(x, y) u=f(x, y), \quad(x, y) \in D \\
u(x, 0)=g(x), \quad 0 \leq x<\infty \\
u(\phi(y), y)=h(y), \quad 0 \leq y<\infty
\end{array}\right.
$$

where the region $\mathrm{D}$ is given by

$$
D=\{(x, y) \mid \phi(y)<x<\infty, 0<y<\infty\}
$$

and all data are supposed to be $\mathrm{C}^{\infty}$-functions which in addition satisfy the conditions

$$
\left\{\begin{array}{l}
a(x, y)>0, b(x, y)>0 \quad \text { in } \bar{D} \\
a(\phi(y), y)-b(\phi(y), y) \phi^{\prime}(y)>0 \quad \text { for } 0 \leq y<\infty
\end{array}\right.
$$

and

$$
\left\{\begin{array}{l}
\phi(0)=0 \\
\phi^{\prime}(y) \geq 0 \quad \text { for } 0 \leq y<\infty \\
g(0)=h(0) .
\end{array}\right.
$$

Then the function $\mathrm{u}(\mathrm{x}, \mathrm{y})$ belongs to $\mathrm{C}^{1}(\overline{\mathrm{D}})$ iff

$$
\left\{a(0,0)-b(0,0) \phi^{\prime}(0)\right\} g^{\prime}(0)+b(0,0) h^{\prime}(0)+c(0,0) g(0)=f(0,0) \text {. }
$$

In that case the second derivatives of $\mathrm{u}(\mathrm{x}, \mathrm{y})$ exist in $\overline{\mathrm{D}}$ and are continuous, with possible exception of the characteristic through the origin where the second derivatives may exhibit a finite jump.

PROOF. Applying the coordinate transformation

$$
\left\{\begin{array}{l}
\xi=x-\phi(y) \\
\eta=y
\end{array}\right.
$$


to problem (41) we obtain a problem which can be treated in the same way as problem (31) of chapter $\mathrm{V}$ (cf. also the remark following the proof of lemma 1 in chapter $\mathrm{V})$.

The difficulty of not being able to evaluate $\mathrm{L}_{2}[\mathrm{w}]$ can be avoided in the same way as in subsection 2.2 of chapter $v$, namely by considering instead of $(40)$ the modified reduced problem

$$
\left\{\begin{array}{l}
\mathrm{L}_{1}^{(0)}[\tilde{\mathrm{w}}]=\mathrm{f}(\mathrm{x}, \mathrm{y}), \quad(\mathrm{x}, \mathrm{y}) \in \mathrm{D} \\
\tilde{\mathrm{w}}(\mathrm{x}, 0 ; \varepsilon)=\mathrm{g}(\mathrm{x}), \quad 0 \leq \mathrm{x}<\infty \\
\tilde{\mathrm{w}}(\phi(y), \mathrm{y} ; \varepsilon)=\mathrm{H}(\mathrm{y} ; \varepsilon), \quad 0 \leq \mathrm{y}<\infty
\end{array}\right.
$$

where the function $\mathrm{H}(\mathrm{y} ; \varepsilon)$ is defined by

$$
H(y ; \varepsilon)=h(y)-C y \exp \left(-\frac{y}{\varepsilon^{p}}\right), \quad 0 \leq y<\infty,
$$

the constant $\mathrm{C}$ is given by

$$
c=\frac{\left\{a_{1}(0,0)-b_{1}(0,0) \phi^{\prime}(0)\right\} g^{\prime}(0)+b_{1}(0,0) h^{\prime}(0)+c(0,0) g(0)-f(0,0)}{b_{1}(0,0)}
$$

and $\mathrm{p}$ is some positive constant to be determined later on. According to lemma 1 the solution $\tilde{w}(x, y ; \varepsilon)$ of $(42)$ is twice differentiable, so the expression $L_{2}[\tilde{w}]$ is now defined.

We define the remainder term $z(x, y ; \varepsilon)$ by

$$
z(x, y ; \varepsilon)=u(x, y ; \varepsilon)-\tilde{w}(x, y ; \varepsilon)
$$

Then we obtain from (34) and (42)

$$
\left\{\begin{array}{l}
\varepsilon L_{2}[z]+L_{1}^{(\varepsilon)}[z]=-\varepsilon\left\{L_{2}[\tilde{w}]+a_{2}(x, y) \frac{\partial \tilde{w}}{\partial x}+b_{2}(x, y) \frac{\partial \tilde{w}}{\partial y}\right\}, \quad(x, y) \in D \\
z(x, 0 ; \varepsilon)=0, \quad 0 \leq x<\infty \\
z(\phi(y), y ; \varepsilon)=C y \exp \left(-\frac{y}{\varepsilon}\right), \quad 0 \leq y<\infty .
\end{array}\right.
$$


A calculation shows that

$$
\begin{cases}\left\|\mathrm{L}_{2}[\tilde{\mathrm{w}}]\right\|_{\Omega}=0\left(\varepsilon^{\left.-\frac{1}{2} \mathrm{p}\right),}\right. & \varepsilon+0 \\ \left\|\frac{\partial \tilde{\mathrm{w}}}{\partial \mathrm{x}}\right\|_{\Omega}=0\left(\varepsilon^{\frac{1}{2} \mathrm{p}}\right), & \varepsilon \downarrow 0 \\ \left\|\frac{\partial \tilde{\mathrm{w}}}{\partial \mathrm{y}}\right\|_{\Omega}=0\left(\varepsilon^{\frac{1}{2} \mathrm{p}}\right), & \varepsilon \downarrow 0\end{cases}
$$

valid for any compact subset $\Omega \subset \overline{\mathrm{D}}$.

Hence, applying theorem 1 (and the second remark following that theorem) to (44) we obtain

$$
\sup _{\Omega}|z(x, y ; \varepsilon)|=0\left(\varepsilon^{\mu}\right), \quad \varepsilon+0
$$

valid for any compact subset $\Omega \subset \bar{D}$, with

$$
\mu=-\frac{1}{4}+\min \left(1-\frac{1}{2} p, \frac{3}{2} p, \frac{1}{2}+\frac{1}{2} p\right) .
$$

It is easily established that $\mu$ becomes optimal if we choose $p=\frac{1}{2}$. In that case we have $\mu=\frac{1}{2}$, so it follows from (43) and (45) that

$$
u(x, y ; \varepsilon)=\tilde{w}(x, y ; \varepsilon)+o\left(\varepsilon^{\frac{1}{2}}\right), \quad \varepsilon \downarrow 0
$$

uniformly valid in any compact subset $\Omega \subset \bar{D}$.

Since the solutions $w$ and $\tilde{w}$ of $(40)$ and (42) satisfy

$$
\tilde{w}(x, y ; \varepsilon)=w(x, y)+0\left(\varepsilon^{\frac{1}{2}}\right), \quad \varepsilon+0
$$

uniformly in each compact subset $\Omega \subset \bar{D}$ (cf. chapter $V$, subsection 2.2) we can formulate

THEOREM 2. Let $\mathrm{u}(\mathrm{x}, \mathrm{y} ; \varepsilon)$ be the solution of the characteristic boundary value problem (34) where all data are $C^{\infty}$-functions which in addition satisfy (35), (36) and (39). Then we have

$$
\mathrm{u}(\mathrm{x}, \mathrm{y} ; \varepsilon)=\mathrm{w}(\mathrm{x}, \mathrm{y})+\mathrm{O}\left(\varepsilon^{\frac{1}{2}}\right), \quad \varepsilon+0
$$

uniformly valid in any compact subset $\Omega \subset \overline{\mathrm{D}}$, where $\mathrm{w}(\mathrm{x}, \mathrm{y})$ is the solution 
of the reduced problem (40).

\section{Approximations for the case with boundary layer along $x=\phi(y), y \geq 0$}

In this section we shall construct an approximation for the solution $\mathrm{u}(\mathrm{x}, \mathrm{y} ; \varepsilon)$ of the characteristic boundary value problem (34) where all data are $c^{\infty}$-functions which in addition satisfy

(46)

$$
\left\{\begin{array}{l}
a_{1}(x, y)>0, \quad b_{1}(x, y)>0 \quad \text { in } \bar{D} \\
a_{1}(\phi(y), y)-b_{1}(\phi(y), y) \phi^{\prime}(y)<0 \quad \text { for } 0 \leq y<\infty
\end{array}\right.
$$

and

$$
\left\{\begin{array}{l}
\phi(0)=0 \\
\phi^{\prime}(y)>0 \quad \text { for } 0 \leq y<\infty \\
g(0)=h(0) .
\end{array}\right.
$$

REMARK. It follows from (46) that the part of the boundary $\partial D$ given by $x=\phi(y), y \geq 0$ can no longer be characteristic. Therefore we have to restrict ourselves to problems with a timelike boundary $x=\phi(y), y \geq 0$. Problems of this type are sometimes re:erred to as Goursat problems.

As a first approximation for $u(x, y ; \varepsilon)$ we take the solution $w_{1}(x, y)$ of the reduced problem

$$
\left\{\begin{array}{l}
\mathrm{L}_{1}^{(0)}\left[\mathrm{w}_{1}\right]=f(x, y), \quad(x, y) \in D \\
w_{1}(x, 0)=g(x), \quad 0 \leq x<\infty
\end{array}\right.
$$

(see figure $4 \mathrm{~b}$ ). In order to cope with the remaining boundary condition we look for an approximation $\tilde{u}$ of $u$ in the form

$$
\tilde{u}(x, y ; \varepsilon)=w_{1}(x, y)+\varepsilon w_{2}(x, y)+v_{1}(\xi, \eta)+\varepsilon v_{2}(\xi, \eta)+\varepsilon^{2} v_{3}(\xi, \eta)
$$

where 


$$
\xi=\frac{x-\phi(y)}{\varepsilon}, \quad \eta=y
$$

and the functions $\mathrm{w}_{2}, \mathrm{v}_{1}, \mathrm{v}_{2}$ and $\mathrm{v}_{3}$ have to be determined.

Applying the transformation $(50)$ to the operator $\mathrm{L}^{(\varepsilon)}=\varepsilon \mathrm{L}_{2}+\mathrm{L}_{1}^{(\varepsilon)}$ and expanding this operator into powers of $\varepsilon$ we obtain after some calculations

$$
L^{(\varepsilon)}=\frac{1}{\varepsilon}\left\{-\phi^{\prime}(\eta) \frac{\partial^{2}}{\partial \xi^{2}}+\lambda_{1}^{(0)}(\eta) \frac{\partial}{\partial \xi}\right\}+M_{0}+\varepsilon M_{1}+\varepsilon^{2} M_{2}^{(\varepsilon)}
$$

where the operators $\mathrm{M}_{0}, \mathrm{M}_{1}$ and $\mathrm{M}_{2}^{(\varepsilon)}$ are defined by

$$
\left\{\begin{aligned}
M_{0}= & \frac{\partial^{2}}{\partial \xi \partial \eta}+\left\{\xi \lambda_{1}^{(1)}(n)+\lambda_{2}^{(0)}(n)\right\} \frac{\partial}{\partial \xi}+b_{1}(\phi(n), n) \frac{\partial}{\partial \eta}+c(\phi(n), \eta) \\
M_{1}= & \left\{\frac{1}{2} \xi^{2} \lambda_{1}^{(2)}(n)+\xi \lambda_{2}^{(1)}(n)\right\} \frac{\partial}{\partial \xi}+\left\{\xi \frac{\partial}{\partial x}(\phi(n), n)+b_{2}(\phi(n), n)\right\} \frac{\partial}{\partial \eta} \\
& +\xi \frac{\partial c}{\partial x}(\phi(n), n) \\
M_{2}^{(\varepsilon)}= & \frac{1}{6} \xi^{3}\left\{\frac{\partial^{3} a_{1}}{\left.\partial x^{3}\left(\phi(n)+\varepsilon \Theta_{1} \xi, n\right)-\phi^{\prime}(\eta) \frac{\partial^{3} b_{1}}{\partial x^{3}}\left(\phi(n)+\varepsilon \Theta_{2} \xi, \eta\right)\right\} \frac{\partial}{\partial \xi}}\right. \\
& +\frac{1}{2} \xi^{2}\left\{\frac{\partial^{2} a}{\partial x^{2}}\left(\phi(n)+\varepsilon \Theta_{3} \xi, \eta\right)-\phi^{\prime}(n) \frac{\partial^{2} b_{2}}{\partial x^{2}}\left(\phi(n)+\varepsilon \Theta_{4} \xi, n\right)\right\} \frac{\partial}{\partial \xi} \\
& +\left\{\frac{1}{2} \xi^{2} \frac{\partial^{2} b_{1}}{\partial x^{2}}\left(\phi(n)+\varepsilon \Theta_{5} \xi, \eta\right)+\xi \frac{\partial b_{2}}{\partial x}\left(\phi(n)+\varepsilon \Theta_{6} \xi, n\right)\right\} \frac{\partial}{\partial \eta} \\
& +\frac{1}{2} \xi^{2} \frac{\partial^{2} c}{\partial x^{2}\left(\phi(n)+\varepsilon \Theta_{7} \xi, n\right)}
\end{aligned}\right.
$$

with $0<\theta_{i}<1(i=1,2, \ldots, 7)$. The functions $\lambda_{i}^{(j)}(n)$ are given by

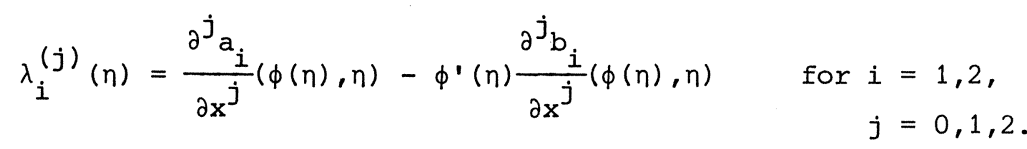

Hence it follows that 
(51)

$$
\begin{aligned}
L^{(\varepsilon)}[\tilde{u}]= & L^{(\varepsilon)}\left[w_{1}+\varepsilon w_{2}\right]+L^{(\varepsilon)}\left[v_{1}+\varepsilon v_{2}+\varepsilon^{2} v_{3}\right] \\
= & L_{1}^{(0)}\left[w_{1}\right]+\varepsilon\left\{L_{1}^{(0)}\left[w_{2}\right]+\frac{\partial^{2} w_{1}}{\partial x \partial y}+a_{2}(x, y) \frac{\partial w_{1}}{\partial x}+b_{2}(x, y) \frac{\partial w_{1}}{\partial y}\right\} \\
& +\frac{1}{\varepsilon}\left\{-\phi^{\prime}(\eta) \frac{\partial^{2} v_{1}}{\partial \xi^{2}}+\lambda_{1}^{(0)}(\eta) \frac{\partial v_{1}}{\partial \xi}\right\} \\
& +\left\{-\phi^{\prime}(\eta) \frac{\partial^{2} v_{2}}{\partial \xi^{2}}+\lambda_{1}^{(0)}(\eta) \frac{\partial v_{2}}{\partial \xi}+M_{0}\left[v_{1}\right]\right\} \\
& +\varepsilon\left\{-\phi^{\prime}(\eta) \frac{\partial^{2} v_{3}}{\partial \xi^{2}}+\lambda_{1}^{(0)}(\eta) \frac{\partial v_{3}}{\partial \xi}+M_{1}\left[v_{1}\right]+M_{0}\left[v_{2}\right]\right\} \\
& +\varepsilon^{2} R(x, y ; \varepsilon)
\end{aligned}
$$

with

$$
\begin{aligned}
R(x, y ; \varepsilon)= & \frac{\partial^{2} w_{2}}{\partial x \partial y}+a_{2}(x, y) \frac{\partial w_{2}}{\partial x}+b_{2}(x, y) \frac{\partial w_{2}}{\partial y} \\
& +M_{2}^{(\varepsilon)}\left[v_{1}\right]+M_{1}\left[v_{2}\right]+\varepsilon M_{2}^{(\varepsilon)}\left[v_{2}\right] \\
& +M_{0}\left[v_{3}\right]+\varepsilon M_{1}\left[v_{3}\right]+\varepsilon^{2} M_{2}^{(\varepsilon)}\left[v_{3}\right] .
\end{aligned}
$$

We now take for the functions $w_{2}, v_{1}, v_{2}$ and $v_{3}$ the solutions of the following problems

$$
\left\{\begin{array}{l}
\mathrm{L}_{1}^{(0)}\left[\mathrm{w}_{2}\right]=-\frac{\partial^{2} \mathrm{w}_{1}}{\partial x \partial y}-\mathrm{a}_{2}(\mathrm{x}, \mathrm{y}) \frac{\partial \mathrm{w}_{1}}{\partial \mathrm{x}}-\mathrm{b}_{2}(\mathrm{x}, \mathrm{y}) \frac{\partial \mathrm{w}_{1}}{\partial \mathrm{y}}, \quad(\mathrm{x}, \mathrm{y}) \in \mathrm{D} \\
\mathrm{w}_{2}(\mathrm{x}, 0)=0, \quad 0 \leq \mathrm{x}<\infty
\end{array}\right.
$$

(53) $\begin{cases}\phi^{\prime}(n) \frac{\partial^{2} v_{1}}{\partial \xi^{2}}-\lambda_{1}^{(0)}(\eta) \frac{\partial v_{1}}{\partial \xi}=0, & 0<\xi<\infty, 0 \leq n<\infty \\ v_{1}(0, n)=h(n)-w_{1}(\phi(n), n), & 0 \leq n<\infty \\ \lim _{\xi \rightarrow \infty} v_{1}(\xi, n)=0, & 0 \leq n<\infty\end{cases}$ 
(54)

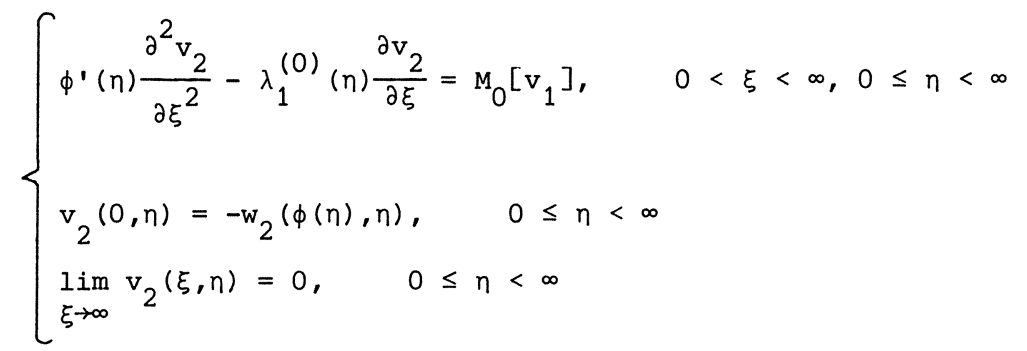

(55)

$$
\left\{\begin{array}{l}
\phi^{\prime}(\eta) \frac{\partial^{2} v_{3}}{\partial \xi^{2}}-\lambda_{1}^{(0)}(n) \frac{\partial v_{3}}{\partial \xi}=M_{1}\left[v_{1}\right]+M_{0}\left[v_{2}\right], \quad 0<\xi<\infty, 0 \leq n<\infty \\
v_{3}(0, n)=0, \quad 0 \leq n<\infty \\
\lim _{\xi \rightarrow \infty} v_{3}(\xi, \eta)=0, \quad 0 \leq n<\infty .
\end{array}\right.
$$

One easily verifies that

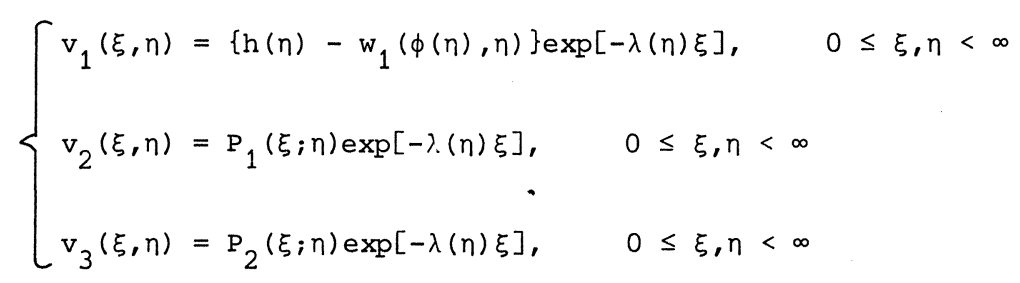

where the positive function $\lambda(n)$ is defined by

$$
\lambda(n)=-\frac{\lambda_{1}^{(0)}(n)}{\phi^{\prime}(n)}=b_{1}(\phi(n), n)-\frac{a_{1}(\phi(n), n)}{\phi^{\prime}(n)} \quad \text { for } 0 \leq n<\infty
$$

and $P_{1}$ and $P_{2}$ are polynomials in $\xi$ with coefficients depending on $\eta$. So $\mathrm{v}_{1}, \mathrm{v}_{2}$ and $\mathrm{v}_{3}$ are boundary layer furctions.

With the above mentioned choice for the functions $w_{i}(i=1,2)$ and $v_{i}$ $(i=1,2,3)$ we obtain from $(48),(49),(51),(52),(53),(54)$ and (55)

(56) $\left\{\begin{array}{l}\mathrm{L}^{(\varepsilon)}[\tilde{u}]=f(x, y)+\varepsilon^{2} R(x, y ; \varepsilon), \quad(x, y) \in D \\ \tilde{u}(x, 0 ; \varepsilon)=g(x)+\varepsilon v_{2}\left(\frac{x}{\varepsilon}, 0\right)+\varepsilon^{2} v_{3}\left(\frac{x}{\varepsilon}, 0\right), \quad 0 \leq x<\infty \\ \tilde{u}(\phi(y), y ; \varepsilon)=h(y), \quad 0 \leq y<\infty .\end{array}\right.$ 
Defining the remainder term $z(x, y ; \varepsilon)$ by

$$
z(x, y ; \varepsilon)=u(x, y ; \varepsilon)-\tilde{u}(x, y ; \varepsilon)
$$

we get from (34) and (56)

$$
\left\{\begin{array}{l}
L^{(\varepsilon)}[z]=-\varepsilon^{2} R(x, y ; \varepsilon), \quad(x, y) \in D \\
z(x, 0 ; \varepsilon)=-\varepsilon v_{2}\left(\frac{x}{\varepsilon}, 0\right)-\varepsilon^{2} v_{3}\left(\frac{x}{\varepsilon}, 0\right), \quad 0 \leq x<\infty \\
z(\phi(y), y ; \varepsilon)=0, \quad 0 \leq y<\infty .
\end{array}\right.
$$

One easily verifies that

$$
\|R\|_{\Omega}=0(1), \quad \varepsilon \downarrow 0
$$

valid for every compact subset $\Omega \subset \bar{D}$.

Hence, applying theorem 1 (and the first remark following that theorem) to (58) we obtain

$$
\mathrm{z}(\mathrm{x}, \mathrm{y} ; \varepsilon)=O(\varepsilon), \quad \varepsilon+0
$$

uniformly valid in any compact subset $\Omega \subset \overline{\mathrm{D}}$.

Finally, using the formulae (49), (57) and (59) and also the fact that the functions $\mathrm{w}_{2}, \mathrm{v}_{2}$ and $\mathrm{v}_{3}$ are uniformly bounded in compact subsets of $\overline{\mathrm{D}}$, we arrive at

THEOREM 3. Let $\mathrm{u}(\mathrm{x}, \mathrm{y} ; \varepsilon)$ be the solution of the Goursat problem (34) where all data are $C^{\infty}$-functions which in addition satisfy (46) and (47). Then we have

$$
\mathrm{u}(\mathrm{x}, \mathrm{y} ; \varepsilon)=\mathrm{w}(\mathrm{x}, \mathrm{y})+\mathrm{o}(\varepsilon), \quad \varepsilon \downarrow 0
$$

uniformly valid in any compact subset

$$
\Omega \subset\{(\mathrm{x}, \mathrm{y}) \mid \phi(\mathrm{y})+\delta \leq \mathrm{x}<\infty, 0 \leq \mathrm{y}<\infty\} .
$$


Here $\mathrm{w}(\mathrm{x}, \mathrm{y})$ is the solution of the reduced problem

$$
\left\{\begin{array}{l}
L_{1}^{(0)}[w]=f(x, y), \quad(x, y) \in D \\
w(x, 0)=g(x), \quad 0 \leq x<\infty
\end{array}\right.
$$

and $\delta$ is an arbitrarily small positive constant independent of $\varepsilon$. In addition we have

$$
u(x, y ; \varepsilon)=w(x, y)+\{h(y)-w(\phi(y), y)\} \exp \left[\lambda(y) \frac{\phi(y)-x}{\varepsilon}\right]+0(\varepsilon), \quad \varepsilon+0
$$

uniformly valid in any compact subset $\Omega \subset \bar{D}$, where the positive function $\lambda(\mathrm{y})$ is defined by

$$
\lambda(y)=b_{1}(\phi(y), y)-\frac{a_{1}(\phi(y), y)}{\phi^{\prime}(y)} \quad \text { for } 0 \leq y<\infty .
$$




\section{REFERENCES}

[1] AGMON, S., Lectures on elliptic boundary value problems, Van Nostrand, New York, 1965.

[2] BESJES, J.G., Singular perturbation problems for partial differential equations, thesis, Technical University Delft, 1973.

[3] BLONDEL, J.M., Etude de deux problèmes singuliers, relatifs aux équations aux dérivées partielles, linéaires et hyperboliques, du second ordre, J. Math. Pures Appl. (9) 40 (1961) pp. 247-299.

[4] BLONDEL, J.M., Perturbation singulière pour une équation aux dérivées partielles du second ordre, linéaire, du type hyperbolique normal, C.R. Acad. Sc. Paris 257 (1963) pp. 353-355.

[5] BURGERS, J.M., The nonlinear diffusion equation, Reidel, Dordrecht, 1974 .

[6] COLE, J.D., Perturbation methods in applied mathematics, Blaisdell, Waltham, Mass., 1968.

[7] DZAVADOV, M.G., The Cauchy problem for a hyperbolic equation with a small parameter multiplying the highest derivatives, Izv. Akad. Nauk Azerb. SSR, Ser. Fiz.-Tek. Mat. Nauk $\underline{6}$ (1963) pp. 3-9.

[8] DZAVADOV, M.G., A mixed problem for a hyperbolic equation involving a small parameter with the leading derivatives, Soviet Math. Doklady 4" [151-153] (1963) pp. 1400-1404.

[9] ECKHAUS, W., Boundary layers in linear elliptic'singular perturbation problems, SIAM Review 14 (1972) pp. 225-270.

[10] ECKHAUS, W., Matched asymptotic expansions and singular perturbations, North-Holland, Amsterdam, 1973.

[11] ECKHAUS, W., Formal approximations and singular perturbations, SIAM Review 19 (1977) pp. 593-633.

[12] ECKHAUS, W. \& E.M. DE JAGER, Asymptotic solutions of singular perturbation problems for linear differential equations of elliptic type, Arch. Rat. Mech. Anal. 23 (1966) pp. 26-86.

[13] FRIEDRICHS, K.o., Symmetric hyperbolic linear differential equations, Comm. Pure Appl. Math. 7 (1954) pp. 345-392. 
[14] FRIEDRICHS, K.O., Asymptotic phenomena in mathematical physics, Bull. Amer. Math. Soc. 61 (1955) pp. 485-504.

[15] FRIEDRICHS, K. \& H. LEWY, Über die Eindeutigkeit und das Abhängigkeitsgebiet der Lösungen beim Anfangswertproblem linearer hyperbolischer Differentialgleichungen, Math. Ann. $\underline{98}$ (1928) pp. 192-204.

[16] GARABEDIAN, P.R., Partial differential equations, Wiley, New York, 1964 .

[17] GENET, J. \& M. MADAUNE, Perturbations singulières pour une classe de problèmes hyperboliques non linéaires; Proceedings of the conference on singular perturbations and boundary layer theory, Lyon, 1976, Lecture Notes in Math. 594, Springer, Berlin, 1977, pp. 201-230.

[18] GENET, J. \& G. PUPION, Perturbation singulière pour un problème mixte relatif à une équation aux dérivées partielles linéaire hyperbolique du second ordre, C.R. Acad. Sc. Paris 261 (1965) pp. 3934-3937, 266 (1968) pp. 489-492, 266 (1968) pp. 658-660.

[19] GRADSTEIN, I.S., Linear equations with variable coefficients and small parameters in the highest derivatives, Amer. Math. Soc. Transl. (1) $\underline{4}$ (1962) pp. 300-330.

[20] GRASMAN, J., On the birth of boundary layers, tract 36, Mathematisch Centrum, Amsterdam, 1971.

[21] DE GROEN, P.P.N., Singularly perturbed differential operators of second order, tract 68, Mathematisch Centrum, Amsterdam, 1976.

[22] HADAMARD, J., Sur l'intégrale résiduelle, Bull. Soc. Math. France 28 (1900) pp. 69-90.

[23] VAN HARTEN, A., Singularly perturbed non-linear 2nd order elliptic boundary value problems, thesis, University of Utrecht, 1975.

[24] HOPPENSTEADT, F.C., Singular perturbations on the infinite interval, Trans. Amer. Math. Soc. 123 (1966) pp. 521-535.

[24 $\left.{ }^{\star}\right]$ HUET, D. , Phénomènes de perturbations singulières dans les problèmes aux limites, Ann. Institut Fourier, Grenoble, 10 (1960) pp. $1-96$. 
[25] DE JAGER, E.M., Singular elliptic perturbations of vanishing firstorder differential operators, Lecture Notes in Math. 280, Springer, Berlin, 1972, pp. 73-86.

[26] DE JAGER, E.M., Singular perturbations of hyperbolic type, Nieuw Arch. Wisk. (3) 23 (1975) pp. 145-172.

[27] LAX, P.D., The formation and decay of shock waves, Amer. Math. Monthly 79 (1972) pp. 227-241.

[28] LIONS, J.L., Perturbations singulières dans les problèmes aux limites et en contrôle optimal, Lecture Notes in Math. 323, Springer, Berlin, 1973.

[29] LIONS, J.L., Quelques méthodes de résolution des problèmes aux limites non Iinéaires, Dunod, Paris, 1969.

[30] MADAUNE, M., Perturbations singulières de type hyperbolique-hyperbolique non linéaire, Publ. Math. de Pau, 1977.

[31] MORAWETZ, C.S., A uniqueness theorem for Frankl's problem, Comm. Pure Appl. Math. 7 (1954) pp. 697-703.

[32] NAYFEH, A., Perturbation methods, Ẉiley, New York, 1973.

[33] OLEINIK, O.A., Discontinuous solutions of non-linear differential equations, Amer. Math. Soc. Transl. (2) 26 (1963) pp. 95-172.

[34] OLEINIK, O.A.; Construction of a generalized solution of the Cauchy problem for a quasi-linear equation of first order by the introduction of "vanishing viscosity", Amer. Math. Soc. Transl. (2) 33 (1963) pp. 277-283.

[35] OLEINIK, O.A., On the uniqueness of the generalized solution of the Cauchy problem for a nonlinear system of equations occurring in mechanics, Uspehi Mat. Nauk 78 (1957) pp. 169-176.

[36] OLEINIK, O.A., Uniqueness and stability of the generalized solution of the Cauchy problem for a quasi-linear equation, Amer. Math. Soc. Transl. (2) 33 (1963) pp. 285-290.

[37] O'MALLEY, JR., R.E., Topics in singular perturbations, Advances in Math. $\underline{2}$ (1968) pp. 365-470.

[38] O'MALLEY, JR., R.E., Introduction to singular perturbations, Academic Press, New York, 1974. 
[39] PONZO, P.J. \& N. WAX, Relaxation oscillations, parasitics, and singular perturbations, IEEE Trans. CT-19 (1972) pp. 623-625.

[40] PROTTER, M.H., Uniqueness theorems for the Tricomi problem, J. Rat. Mech. Anal. 2 (1953) pp. 107-114.

[41] ROSEAU, M., Asymptotic wave theory, North-Holland, Amsterdam, 1976.

[42] RUBINOWICZ, A., Herstellung von Lösungen gemischter Randwertprobleme bei hyperbolischen Differentialgleichungen zweiter ordnung durch Zusammenstückelung aus Lösungen einfacherer gemischter Randwertaufgaben, Monatsh. Math. Phys. 30 (1920) p. 65.

[43] RUBINSTEIN, Z., A course in ordinary and partial differential equations, Academic Press, New York, 1969.

[44] SMITH, D.R., An asymptotic analysis of a certain hyperbolic Cauchy problem, SIAM J. Math. Anal. 2 (1971) pp. 375-392.

[45] SMITH, D.R., The multivariable method in singular perturbation analysis, SIAM Review 17 (1975) pp. 221-273.

[46] SMITH, D.R. \& J.T. PALMER, On the behavior of the solution of the telegraphist's equation for large absorption, Arch. Rat. Mech. Anal. 39 (1970) pp. 146-157.

[47] VASIL'EVA, A.B., Asymptotic behaviour of solutions to certain problems involving nonlinear differential equations containing a small parameter multiplying he highest derivatives, Russian Math. Surveys 18 (1963) pp. 13-84.

[48] VASIL'EVA, A.B. \& V.M. VOLOSOV, The work of Tikhonov and his pupils on ordinary differential equations containing a small parameter, Russian Math. Surveys $\underline{22}$ (1967) pp. 124-142.

[49] VISIK, M.I. \& L.A. LYUSTERNIK, Regular degeneration and boundary layer for linear differential equations with small parameter, Amer. Math. Soc. Transl. (2) 20 (1952) pp. 239-364.

[50] volosov, V.M., On the theory of nonlinear differential equations of higher orders with a small parameter in the highest derivative, Amer. Math. Soc. Transl. (2) $\underline{8}$ (1958) pp. 209-241.

[51] VOLOSOV, V.M., On solutions of some differential equations of the second order depending upon a parameter, Amer. Math. Soc. Transl. (2) 8 (1958) pp. 243-255. 
[52] WASOW, W., Asymptotic expansions for ordinary differential equations, Interscience, New York, 1965.

[53] WEBER, H., Die partiellen Differentialgleichungen der mathematischen Physik nach Riemann's Vorlesungen in 4ter Auflage neu bearbeitet, Vieweg, Braunschweig, 1900.

[54] WEINSTEIN, M.B. \& D.R. SMITH, Comparison techniques for overdamped systems, SIAM Review 17 (1975) pp. 520-540.

[55] WEINSTEIN, M.B. \& D.R. SMITH, Comparison techniques for certain overdamped hyperbolic partial differential equations, Rocky Mountain J. Math. 6 (1976) pp: 731-742.

[56] WHIthAM, G.B., Linear and nonlinear waves, Wiley, New York, 1974.

[57] ZAREMBA, S., Sopra un teorema d'unicità relativo alla equazione delle onde sferiche, Rend. Accad. Naz. Lincei (5) 24 (1915) pp. 904-908. 
OTHER TITLES IN THE SERIES MATHEMATICAL CENTRE TRACTS

A leaflet containing an order-form and abstracts of all publications mentioned below is available at the Mathematisch Centrum, Tweede Boerhaavestraat 49, Amsterdam-1005, The Netherlands. Orders should be sent to the same address.

MCT 1 T. VAN DER WALT, Fixed and almost fixed points, 1963. ISBN 906196 0029.

MCT 2 A.R. BLOEMENA, Sampling from a graph, 1964. ISBN 9061960037.

MCT 3 G. DE LEVE, Generalized Markovian decision processes, part I: Model and method, 1964. ISBN 9061960045.

MCT 4 G. DE LEVE, Generalized Markovian decision processes, part II: Probabilistic background, 1964. ISBN 9061960053 .

MCT 5 G. DE LEVE, H.C. TIJMS \& P.J. WEEDA, Generalized Markovian decision processes, Applications, 1970. ISBN 9061960517.

MCT 6 M.A. MAURICE, Compact ordered spaces, 1964. ISBN 9061960061.

MCT 7 W.R. VAN ZWET, Convex transformations of random variables, 1964. ISBN $906196007 \mathrm{x}$.

MCT 8 J.A. ZONNEVELD, Automatic numerical integration, 1964. ISBN 906196 0088.

MCT 9 P.C. BAAYEN, Universal morphisms, 1964. ISBN 9061960096.

MCT 10 E.M. DE JAGER, Applications of distributions in mathematical physics, 1964. ISBN $906196010 \mathrm{x}$.

MCT 11 A.B. PAALMAN-DE MIRANDA, Topological semigroups, 1964. ISBN 906196 0118.

MCT 12 J.A.TH.M. VAN BERCKEL, H. BRANDT CORSTIUS, R.J. MOKKEN \& A. VAN WIJNGAARDEN, Formal properties of newspaper Dutch, 1965. ISBN 9061960134 .

MCT 13 H.A. LAUWERIER, Asymptotic expansions, 1966, out of print; replaced by MCT 54 and 67.

MCT 14 H.A. LAUWERIER, Calculus of variations in mathematical physics, 1966. ISBN 9061960207.

MCT 15 R. DOORNBOS, Slippage tests, 1966. ISBN 9061960215.

MCT 16 J.W. DE BAKKER, Formal definition of programing languages with an application to the definition of ALGOL 60, 1967. ISBN 906196 0223.

MCT 17 R.P. VAN DE RIET, Formula manipulation in ALGOL 60, part 1, 1968. ISBN 9061960258.

MCT 18 R.P. VAN DE RIET, Formula manipulation in ALGOL 60, part 2, 1968. ISBN $906196038 \mathrm{x}$.

MCT 19 J. VAN DER SLOT, Some properties related to compactness, 1968. ISBN 9061960266 .

MCT 20 P.J. VAN DER HOUWEN, Finite difference methods for solving partial differential equations, 1968. ISBN 9061960274 . 
MCT 21 E. WATTEL, The compactness operator in set theory and topology, 1968. ISBN 9061960282 .

MCT 22 T.J. DEKKER, ALGOL 60 procedures in numerical algebra, part 1, 1968. ISBN 9061960290 .

MCT 23 T.J. DEKKER \& W. HOFFMANN, ALGOL 60 procedures in numerical algebra, part 2, 1968. ISBN 9061960304.

MCT 24 J.W. DE BAKKER, Recursive procedures, 1971. ISBN 9061960606.

MCT 25 E.R. PAERL, Representations of the Lorentz group and projective geometry, 1969. ISBN 9061960398.

MCT 26 EUROPEAN MEETING 1968, Selected statistical papers, part I, 1968. ISBN 9061960312 .

MCT 27 EUROPEAN MEETING 1968, Selected statistical papers, part II, 1969. ISBN 9061960401.

MCT 28 J. DOSTERHOFF, Combination of one-sided statistical tests, 1969. ISBN $906196041 \mathrm{x}$.

MCT 29 J. VERHOEFF, Error detecting decimal codes, 1969. ISBN 9061960428.

MCT 30 H. BRANDT CORSTIUS, Excercises in computational linguistics, 1970. ISBN 9061960525.

MCT 31 W. MOLENAAR, Approximations to the Poisson, binomial and hypergeometric distribution functions, 1970. ISBN 9061960533.

MCT 32 L. DE HAAN, On regular variation and its application to the weak convergence of sample extremes, 1970. ISBN 9061960541.

MCT 33 F.W. STEUTEL, Preservation of infinite divisibility under mixing and related topics, 1970. ISBN 9061960614.

MCT 34 I. JUHÁSZ, A. VERBEEK \& N.S. KROONENBERG, Cardinal functions in topology, 1971. ISBN 9061960622 .

MCT 35 M.H. VAN EMDEN, An analysis of complexity, 1971. ISBN 9061960630 .

MCT 36 J. GRASMAN, On the birth of boundary Zayers, 1971. ISBN 9061960649.

MCT 37 J.W. DE BAKKER, G.A. BLAAUW, A.J.W. DUIJVESTIJN, E.W. DIJKSTRA, P.J. VAN DER HOUWEN, G.A.M. KAMSTEEG-KEMPER, F.E.J. KRUSEMAN ARETZ, W.L. VAN DER POEL, J.P. SCHAAP-KRUSEMAN, M.V. WILKES \& G. ZOUTENDIJK, MC-25 Informatica Symposium, 1971. ISBN 9061960657.

MCT 38 W.A. VERLOREN VAN THEMAat, Automatic analysis of Dutch compound words, 1971. ISBN 9061960738.

MCT 39 H. BAVINCK, Jacobi series and approximation, 1972. ISBN 9061960746.

MCT 40 H.C. TIJMS, Analysis of $(s, S)$ inventory models, 1972. ISBN 9061960754 .

MCT 41 A. VERBEEK, Superextensions of topological spaces, 1972. ISBN 90 61960762.

MCT 42 W. VERVAAT, Success epochs in Bernoulli trials (with applications in number theory), 1972. ISBN 9061960770.

MCT 43 F.H. RUYMGAART, Asymptotic theory of rank tests for independence, 1973. ISBN 9061960819.

MCT 44 H. BART, Meromorphic operator valued functions, 1973. ISBN 9061960827. 
MCT 45 A.A. BALKEMA, Monotone transformations and limit Zows, 1973. ISBN 9061960835.

MCT 46 R.P. VAN DE RIET, ABC ALGOI, A portable Zanguage for formila manipulation systems, part 1: The language, 1973. ISBN 9061960843.

MCT 47 R.P. VAN DE RIET, ABC ALGOL, A portable language for formula manipulation systems, part 2: The compiler, 1973. ISBN 9061960851.

MCT 48 F.E.J. KRUSEMAN ARETZ, P.J.W. TEN HAGEN \& H.L. OUDSHOORN, An ALGOL 60 compiler in ALGOL 60, Text of the MC-compiler for the $E L-X 8$, 1973. ISBN $906196086 \mathrm{x}$.

MCT 49 H. KoK, Connected orderable spaces, 1974. ISBN 9061960886.

MCT 50 A. VAN WiJNGAaRden, B.J. MAIlloux, J.E.L. PECK, C.H.A. Koster, M. SINTZOFF, C.H. LINDSEY, L.G.L.T. MEERTENS \& R.G. FISKER (Eds), Revised report on the algorithmic language ALGOL 68, 1976. ISBN 906196.0894.

MCT 51 A. HORDIJK, Dynamic programming and Markov potential theory, 1974. ISBN 9061960959.

MCT 52 P.C. BAAYEN (ed.), Topological structures, 1974. ISBN 9061960967.

MCT 53 M.J. FABER, Metrizability in generalized ordered spaces, 1974. ISBN 9061960975.

MCT 54 H.A. LAUWERIER, Asymptotic analysis, part 1, 1974. ISBN 9061960983.

MCT 55 M. HALL JR. \& J.H. VAN LINT (Eds), Combinatorics, part 1: Theory of designs, finite geometry and coding theory, 1974. ISBN 9061960991 .

MCT 56 M. HALL JR. \& J.H. VAN LINT (Eds), Combinatorics, part 2: graph theory, foundations, partitions and combinatorial geometry, 1974. ISBN 9061961009.

MCT 57 M. HALL JR. \& J.H. VAN LINT (Eds), Combinatorics, part 3: Combinatorial group theory, 1974. ISBN 9061961017.

MCT 58 W. ALBERS, Asymptotic expansions and the deficiency concept in statistics, 1975. ISBN 9061961025.

MCT 59 J.L. MIJNHEER, Somple path properties of stabie processes, 1975. ISBN 9061961076.

MCT 60 F. GÖBEL, Queueing models involving buffers, 1975. ISBN 9061961084 .

* MCT 61 P. VAN EMDE BOAS, Abstract resource-bound classes, part 1. ISBN 9061961092 .

* MCT 62 P. VAN EMDE BOAS, Abstract resource-bound classes, part 2. ISBN 9061961106 .

MCT 63 J.W. DE BAKKER (ed.), Foundations of computer science, 1975. ISBN 9061961114 .

MCT 64 W.J. DE SCHIPPER, Symmetric closed categories, 1975. ISBN 906196 1122.

MCT 65 J. DE VRIES, Topological transformation groups 1 A categorical approach, 1975. ISBN 9061961130.

MCT 66 H.G.J. PIJLS, Locally convex algebras in spectral theory and eigenfunction expansions, 1976. ISBN 9061961149. 
* MCT 67 H.A. LAUWERIER, Asymptotic analysis, part 2. ISBN $906196119 \mathrm{x}$.

MCT 68 P.P.N. DE GROEN, Singularly perturbed differential operators of second order, 1976. ISBN 9061961203.

MCT 69 J.K. LENSTRA, Sequencing by enumerative methods, 1977. ISBN 9061961254 .

MCT 70 W.P. DE ROEVER JR., Recursive program schemes: semantics and proof theory, 1976. ISBN 9061961270.

MCT 71 J.A.E.E. VAN NUNEN, Contracting Markov decision processes, 1976. ISBN 9061961297.

MCT 72 J.K.M. JANSEN, Simple periodic and nonperiodic Lamé functions and their applications in the theory of conical waveguides,1977. ISBN 9061961300 .

* MCT 73 D.M.R. LEIVANT, Absoluteness of intuitionistic logic. ISBN $906196122 \mathrm{x}$.

MCT 74 H.J.J. TE RIELE, A theoretical and computational study of generalized aliquot sequences, 1976. ISBN 9061961319.

MCT 75 A.E. BROUWER, Treelike spaces and related connected topological spaces, 1977. ISBN 9061961327.

MCT 76 M. REM, Associons and the closure statement, 1976. ISBN 9061961351.

MCT 77 W.C.M. KALLENBERG, Asymptotic optimality of likelihood ratio tests in exponential families, 1977 ISBN 9061961343.

MCT 78 E. DE JONGE, A.C.M. VAN ROOIJ, Introduction to Riesz spaces, 1977. ISBN 9061961335.

MCT 79 M.C.A. VAN ZUIJLEN, Empirical distributions and rankstatistics, 1977. ISBN 9061961459.

MCT 80 P.W. HEMKER, A numerical study of stiff two-point boundary problems, 1977. ISBN 9061961467 .

MCT 81 K.R. APT \& J.W. DE BAKKER (eds), Foundations of computer science II, part I, 1976. ISBN 9061961408.

MCT 82 K.R. APT \& J.W. DE BAKKER (eds), Foundations of computer science II, part II, 1976. ISBN 9061961416.

* MCT 83 L.S. VAN BENTEM JUTTING, Checking Landau's "Gmundlagen" in the AUTOMATH. system, ISBN 9061961475.

MCT 84 H.L.L. BUSARD, The translation of the elements of Euclid from the Arabic into Latin by Hermann of Carinthia (?) books vii-xii, 1977. ISBN 9061961483.

MCT 85 J. VAN MILL, Supercompactness and WalZman spaces, 1977. ISBN 9061961513.

MCT 86 S.G. VAN DER MEULEN \& M. VELDHORST, TOPMix I, 1978. ISBN 9061961521.

* MCT 87 S.G. VAN DER MEULEN \& M. Veldhorst, Tormix II, ISBN $906196153 \mathrm{x}$.

MCT 88 A. SCHRIJVER, Matroids and Zinking systems, 1977. ISBN 9061961548 . 
MCT 89 J.W. DE ROEVER, Complex Fourier transformation and analytic functionals with unbounded carriers, 1978. ISBN 9061961556.

* MCT 90 L.P.J. GROENEWEGEN, Characterization of optimal strategies in dynamic games, . ISBN 9061961564.

* MCT 91 J.M. GEYSEL, Transcendence in fields of positive characteristic, - ISBN 9061961572 .

* MCT 92 P.J. WEEDA, Finite generalized Markov programing, ISBN 9061961580 .

MCT 93 H.C. TIJMS (ed.) \& J. WESSELS (ed.), Markov decision theory, 1977. ISBN 9061961602 .

MCT 94 A. BIJLSMA, Simultaneous approximations in transcendental number theory, 1978. ISBN 9061961629.

MCT 95 K.M. VAN HEE, Bayesian control of Markov chains, 1978. ISBN 9061961637.

* MCT 96 P.M.B. VITÁNYI, Lindenmayer systems: structure, Zanguages, and growth functions, 1978 . ISBN 9061961645 .

* MCT 97 A. FEDERGRUEN, Markovian control problems; functional equations and algorithms, 1978 . ISBN 9061961653.

MCT 98 R. GEEL, Singular perturbations of hyperbolic type, ISBN 9061961661

MCT 99 J.K. LENSTRA, A.H.G. RINNOOY KAN \& P. VAN EMDE BOAS, Interfaces between computer science and operations research, 1978. ISBN $906196170 \mathrm{x}$.

* MCT 100 P.C. BAAYEN, D. VAN DULST \& J. OOSTERHOFF (Eds), Proceedings bicentennial congress of the Wiskundig Genootschop, part 1, ISBN 9061961688 .

* MCT 101 P.C. BAAYEN, D. VAN DULST \& J. OOSTERHOFF (Eds), Proceedings bicentennial congress of the Wiskundig Genootschop, part 2, ISBN 9091961696.

MCT 102 D. VAN DULST, Reflexive and superreflexive Banach spaces, 1978. ISBN 9061961718.

* MCT $103 \mathrm{~K}$. VAN HARN, Classifying infinitely divisible distributions by functional equations, . ISBN 9061961726. 


\section{-}

